

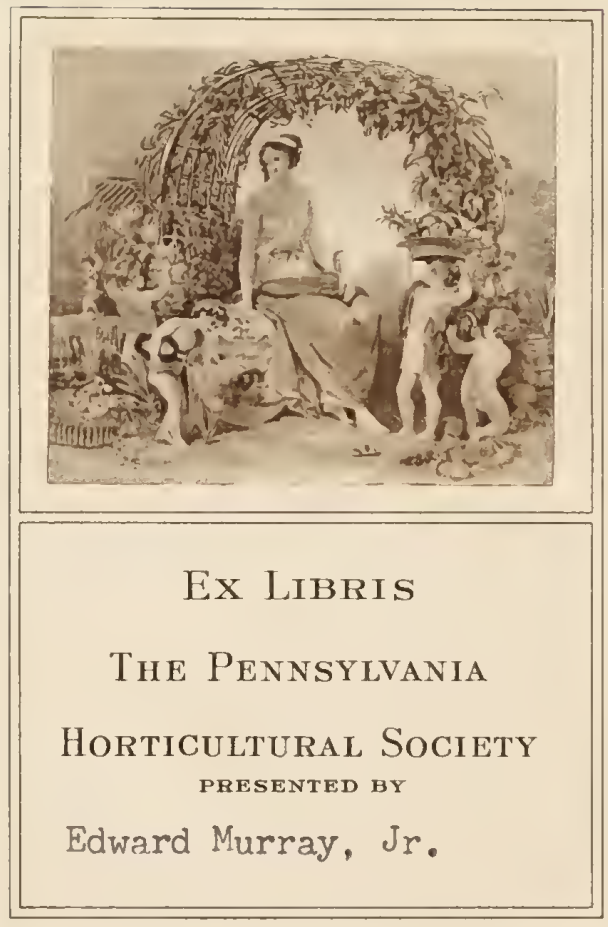




Digitized by the Internet Archive in 2016 



\title{
PLANTÆ NOVÆ
}

\section{VEL MINUS COGNIT $\rightleftarrows$}

EX HERBARIO HORTI THENENSIS

\section{PLANTES NOUVELLES OU PEU CONNUES}

CON'TENUES DANS L'HERBIER

$\mathrm{DE}$

L'HORTUS THENENSIS

AVEC LES DESCRIPTIONS OU ANNOTATIONS

DE

\author{
M. Ém. DE WILDEMAN \\ DOCTEUR EN SCIENCES \\ CONSERVATEUR AU JARDIN BOTANIQUE DE L'ÉTAT
}

\section{Première livraison.}

(MARS 1904)

VEUVE MONNOM

IMPRINIF:UR FI' NIDI'LEUIR

32, RUE DE L'INDUSTRIE, 32

IRRUXELLES

Se vend exclusivement à l'Administration des biens et affaires de M. van den Bossche, 5, Grande Montagne, à Tirlemont. 

PLANTA NOVE

VEL MINUS COGNITA

FX HERBARIO HORTI THENENSIS

PLANTES NOUVELLES OU PEU CONNUES

CONTENUES DANS L'HERBIER DE

L'FIORTUS THENENSIS 
$10260^{\prime 2}$ 


\section{PLANT $Æ$ NOV $Æ$}

\section{VEL MINUS COGNIT $Æ$}

EX IIERBARIO HORTI TIIENENSIS

\section{PLANTES NOUVELLES OU PEU CONNUES}

CONTENUES DANS L'HERBIER

$\mathrm{DE}$

\section{L'HOFTUS THENENSIS}

AVEC LES DESCRIPTIONS OU ANNOTATIONS

$\mathrm{DE}$

M. Ém. DE WILDEMAN

DOCTEUR EN SCIENCES

CONSERVATEY R AU JARDIN BOTANIQUE DE L'ÉTAT

\section{Première livraison.}

(MARS 1904)

VEUVE MONNOM

IMPIRIMF:UIR E'I FIDITHUR

3 ', RUE DE L'INDJSTRIE, 32

BRUXELLES

Se vend exclusivement à l'Administration des biens et affaires de M. van den Bossche, 5, Grande Montagne, à Tirlemont. 



\section{AVANT-PROPOS}

Les Plantce novce vel minus cognita ex Herbario IIorti Thenensis, dont je commence aujourd'hui la publication, formeront un recueil qui, dans mes intentions, est destiné à marcher de pair avec les Icones selectoe.

Ce recueil est destiné à faire connaitre un certain nombre d'espèces nouvelles et, aussi, d'autres espèces dẹ́à déterminées, mais encore inédites, - rencontrées les unes et les autres dans l'Herbier que j’ai formé depuis quelques années pour faciliter l'étude des plantes vivantes de ma collection. Dans certains cas, pour mieux éclairer la valeur des dessins, elles seront présentées en comparaison de types déjà connus, qui n'ont pas été figurés jusqu'à ce jour.

J'ai prié M. le docteur De Wildeman de bien vouloir se charger de ce travail, de me prêter pour les Plantec novce comine pour les Icones selecter le concours éclairé que sa haute compétence et sa gracieuse obligeance me rendent doublement précieux.

Le plan de la nouvelle publication s'écarte quelque peu de celui des Icones. Une description sommaire en latin met d'abord le travail à la portée de tous ceux qui s'occupent de science botanique. Cette description est ensuite développée en français, soit dans l'une des deux langues usuelles en Belgique, et enfin quelques observations complémentaires sont destinées à fixer la place que semblent devoir occuper les espèces nouvelles dans la systématique du gevure ou, éventuellement, de la famille à laquelle elles appartiennent.

Les planches seront encore dessinées par M.A.-R. d'Apre- 
val, dont le talent et l'exactitude ont pu être appréciés à leur valeur.

Les livraisons des Plantee novæe paraîtront sans aucun caractère de périodicité, suivant la valeur des matériaux trouvés dans mon Herbier et, surtout, au gré des loisirs que ses nombreux travaux donneront à M. De Wildeman.

LÉON VAN DEN Bossche.

Tirlemont, le 25 mars 1904. 


\section{PREMIÉRE LIVRAISON}

La première livraison des Plante nove fait connaitre cinq espèces nouvelles rencontrées dans une collection de plantes du Mozambique. Cette collection, qui m’a été cédée par M. Ed. Luja, avait été formée par lui au cours des années 1900 et 1901 , alor's qüil était chef des plintations de Mossumbala (dans la région du Zambèze).

La première des espèces figurées dans les Planta nova. est dédiée à $\mathbf{M}^{\text {me }}$ van den Bossche, qui partagre mon intérêt pour mes collections botaniques et veut hien me prêter, daus la haute surveillance, un concours qui miest surtout précieux pendant les longs jours oì mon état de santé m’oblige à rester enfermó dans mon appartenent.

J'ai voulu, par un sentiment qui sera compris de tous, que la seconde espèce portàt le nom de $\mathbf{M}$. le docteur De Irildeman, et, pour quil puisse en être ainsi, il a bien fallu que je me résigne à la signer de mon nom, tout incapable que je sois d'établir une diagnose scientifique.

L. B. 




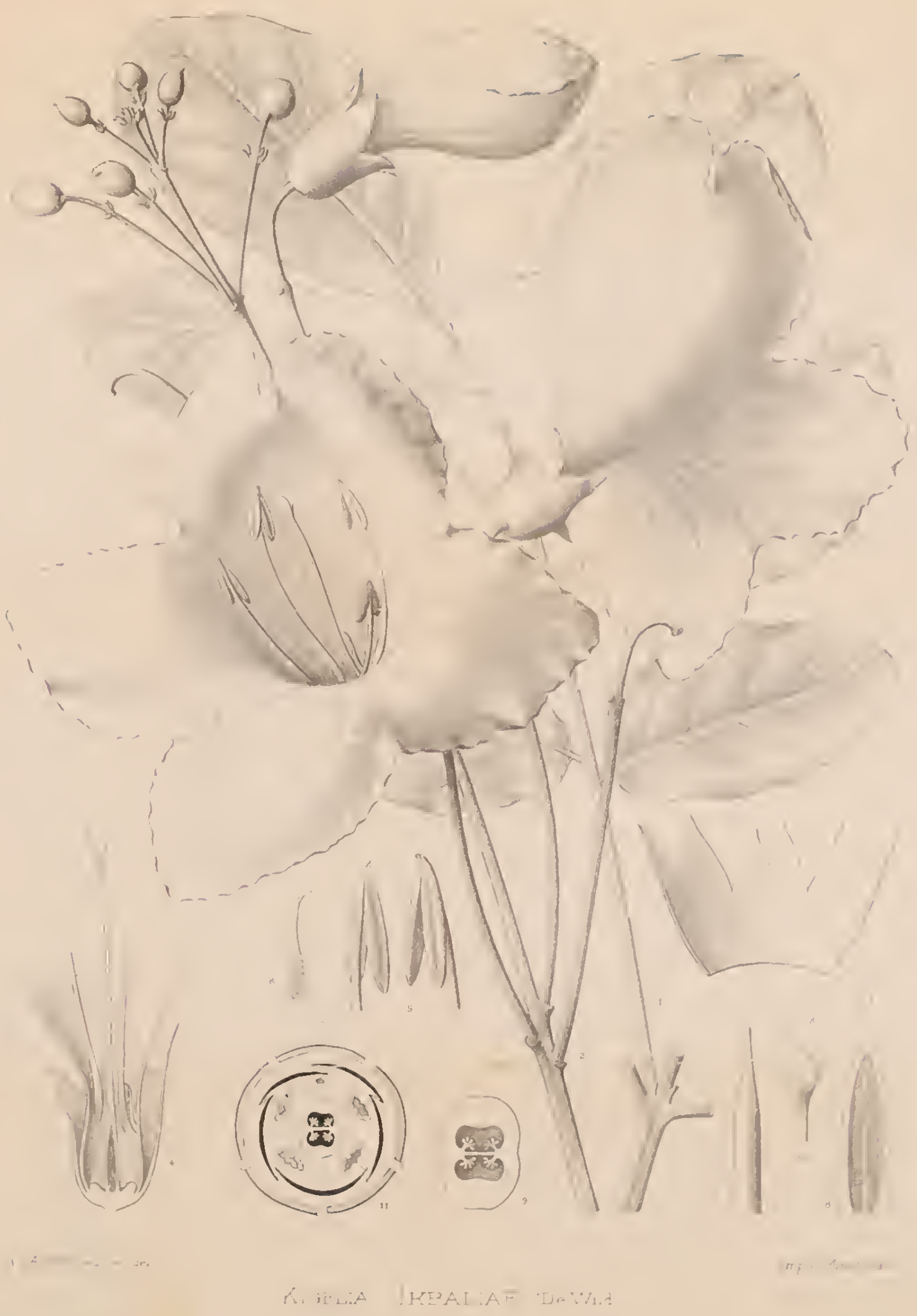




\section{Kigelia Ikbaliæ De Wild. nov. sp.}

(BIGNONIACE 2 )

Kigelia Ikbaliæ; foliis imparipennatis $3-4$ jugis. foliolis ellipticis vel obovato-ellipticis, paulo inæqualibus, terminali obovato, coriaceis, utrinque breviter tomentosis, rugulosis ; panicula longissima, terminali, pedicellis apice reflexis, calice campanulato, coriaceo, extus breviter tomentoso, irregulariter bilabiata, labiis sursum irregulariter fissis; corolla ampla, coriacea, bilabiata, labiis ovatis infer emarginatis; staminibus 4 rudimento 5 subulato; antheris 2 -locularibus, loculis liberis; stylo glabro.

Grand arbre à feuilles imparipennées, à 3 ou 4 paires de folioles; folioles elliptiques ou obovales-elliptiques, légèrement inéquilatérales, atteignant $11 \mathrm{~cm}$. de long et $7,5 \mathrm{~cm}$. de large, obtuses à la base, courtement pétiolulées, la terminale obovale, cunéiforme à la base et obtuse an sommet, courtement apiculées, coriaces, courtement tomenteuses, à poils courts, érigés, denses sur les deux faces. Panicules assez grandes, terminales, à pédicelles réfléchis à l'extrémité. Calice campanulé, coriace, de $3 \mathrm{~cm}$. environ de long, courtement tomenteux extérieurement, surtout à la base, irrégulièrement bilobé, à lobes irrégulièrement lobés. Corolle

\section{Explication des figures de la planche I.}

Fis. 1. - Fragment de rameau avec une feuille de petite taille $(1,1)$.

FIG. 2. - Fragment d'inflorescence (1/1).

Fro. 3. - Calice fendu et élalè (1/1).

Fig. 4. - Coupe longitudinale de la base dune fleur (1/1).

Fig. 5. - Étamines vues de face et de dos (3/1). 
ample, coriace, brunâtre intérieurement, jaunâtre extérieur'ement, de $10 \mathrm{~cm}$. de lonğ, lobe supérieur arrondi au sommet, bilobé, lobe intérieur profondément bilobé, à lobes oblongs, obtus. Etamines fertiles, au nombre de 4, insérées vers la base du tube, à filet grêle, velu à la base, anthères biloculaires, a logres en forme de sacs, libres à la base, glabres; staminode légèrement rentlé à la base, aigu, style glabre environ aussi long que les longues étamines, a deux lobes stigmatiques élargis et aplatis surmontant un ovaire allongé, biloculaire. Fruit très développé, subcylindrique.

Hab. - Moçambique : Chilomo, 6 aoùt 1900 (Éd. Luja, n. 314).

Olservations. - Cette plante aurait également été rencontrée par M. Luja dans l'île d'Inhangana, mais nous ne possédons pas d'échantillons prorenant de cette dernière localité.

Le grenre Ligelia, de la fimulle des Bignoniacen, ne renfermait que trois expèces : Ki.africana Benth., $K$. aethiopica LC. et $K$. madagascariensis Baker; deux de ces especes sont endémiques au continent africaill; la première, plus spéciale à l'Atíique occidentale, la seconde localisée plus ou moins nettement à l'est de la chaine de montagnes qui sépare l'Afrique, au niveau de la région des lacs, en deux parties très différentes au point de vue floral. Cependant, comme nous avons pu le voir par les matériaux rapportés de son séjour au liatanga par le commandant Verdick et quı nous ont servi à publier nos Etudes sur la flore du Katanga, le $\mathbf{K}$. aethiopica se retrouve dans l'Etat Indépendant du Congo à l'ouest, donc à la limite que nous lui assignions plus haut. On peut trouver des renseignements détaillès sur la dispersion de ces especes dans la Revision des Bignoniacées du professeur Ki. Schumann in Engl. et Prantl Pflanzenfam. 4, 3 B p. 249 et dans le Pflanzenw. Ost-Afr. C p. 364. La différenciation des espèces continentales africaines n'est pas dificile. Tandis que le $\boldsymbol{K}$. atricana possède des feuilles

Flu. 6. - Staminode isolé (2/1).

Frti. 7. - Ovaire entier (2/1).

FIo. 8. - Coupe longitudinale de l'ovaile (3/1).

Fig. 9. - Coupe transversale de l'ovaire(4/1).

FIG. 10. - Stigmates (2/1).

FIG. 11. - Diagramme floral. 
à folioles aiguës, le $K$. uethiopic possède des folioles obtuses, comme le montrent très clairement les figures publiées par I)elesser't dans ses Iconcs selcetac vol. V tab. $93 \quad \Lambda$. Le $K$. abyssinica Rich. décrit et figuré dans le 'Tentamen l'lorex Abyssinica, vol. II, p. 60 ct pl. I.XXV doit ètre considéré conme synonyme du $K$. acthiopica dont il possède tons les earactèrcs. I'ailleu's, mêmesi l'on désirait eonserver cette cspèce distincte, elle se différencierait par la glabréité des diverses partics et par la petitesse des folioles.

La plante que nous figurons ici est done très voisine du $K$. aethiopica pris dans son sens le plus large; mais tandis que elıcz le type de I)ccaisne les folioles sont totalement glabres, de mène que les raehis des feuilles et des inflorescences et le calice, chez le $K$. Ikbalia, ces diverses prartics de la plante sont couvertes d'un tomentum raide, court, qui leur donne $11 n$ facies très différent; grâce à l’absenec de villosité des feuilles, la diseolorité des feuilles du $K$. aethiopica cst beaucoup plus aecusée que eellc des feuilles du K. Ikbalice. Les autres caractères spécifiques de ce derniel' sont assez semblables à ceux du $K$. aethiopica; nous ne vondrions pas nous bascr sur la grandeur des folioles, plus développées che\% notre plante que chez l'espèce voisine, n’ayant pu étudier des exemplaires cn nombre suffisant. 




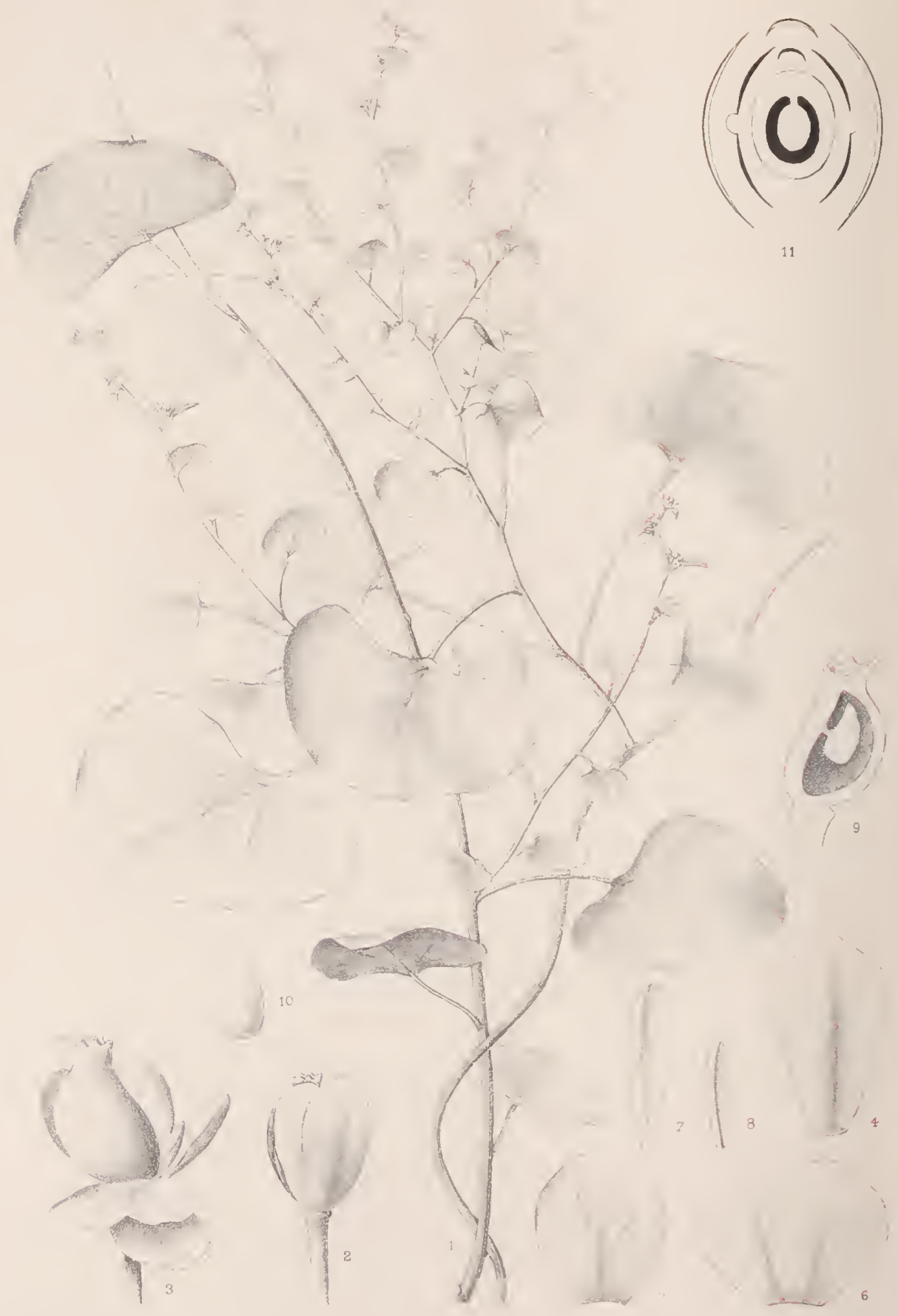


Cissampelos Wildemaniana $v$. 1. Bossche nor. sp.

(MENISPERMACE E)

Gissampelos Wildemaniana, sparse villosa, caule tenui; foliorum petiolo tenuissimo quam lamina subequilongo, lamina peltata, tenuiter membranaeea, semi-ovata, basi truncata, apiee obtusa vel emarginata, breviter apieulata, nervis 5 ab insertione petioli, a basi laminae radiantibus; infloreseentiis axillaribus quam petiolus subaequilongis, eymoso panieulatis tenuissimis, pedieellis tenuissimis; sepalis obovatis emarginatis quam corolla longioribus; ovario glabro, uniovulato, apice lobulato.

Plante grimpante ì tiges grêles, éparsement velues, à feuilles alternes, ì pétiole grêle, environ aussi long que les feuilles, de 10 a $30 \mathrm{~mm}$. de long, sauf sur les ramuscules terminanx et florifères clont les feuilles extrèmes sont parfois très réduites, atteignant $3 \mathrm{~mm}$. de longr senlement, ef subsessiles. Lame foliaire de 1,5 à $4 \mathrm{~cm}$. de large et de 1 à $3.5 \mathrm{~cm}$. de haut, plus réduite dans le voisinage rles inflorescences, peltée, membraneuse, subréniforme, tronquée à la hase, obtuse au sommet ou courtement émarginée, courtement apiculée, à 5 nervures basilaires, radialement disposées à partir du sommet du pétiole, plus proéminentes

\section{Explication des figures de la planche II.}

Fig. 1. - Rameaux enlacés, grandeur naturelle.

FIG. 2. - Bouton de la fleur femelle (13/1).

FiG. 3. - Fleur épanouie (14/1).

FIG. 4. - Bractée externe (18/1).

FIG. 5 et 6 . - Pièces latérales du périgone (18/1). 
en dessous qu'au-dessus. Inflorescences axillaires, en cymes paniculées, pauciflores, dépassées par les feuilles et dépassant légèrement le pétiole. Fleurs femelles pédicellées, à pédicelle grêle, de $2 \mathrm{~mm}$. environ de long, entourées de deux bractées ohovales rapidement caduques, périgone à deux grandes pièces latérales largement elliptiques, entières ou émarginées, et à deux pières postérieures oblongues, plus au moins cucullées, plus étroites que les pièces latérales. Oraire glabre, ovoïde, ohlique, à un senl ovule attuché latéralement, terminé jar un style court, à lobes plus ou moins nombreux et divergents, plus orl moins allongés.

\section{Hab. - Moçambique : dans les forêts du Morrumbala (Zambéze) 1900 (Éd. Luja, n. 473).}

Observations. - L'espèce que nous venons de décrire présente des caractrips assez particulier's : ils ne concordent pas jusqu'à un certain point avec ceux que l'on accepte généralement pour le genre Cissampelos, néanmoins nous n'avons pas osé établir' sur d'uniques fleur's femelles un genre noureau.

Les auteurs décrivent les fleurs femelles des Cissampclos comme coustituées par "I sépale ou bractéole et 1 pétale ou sépale entier ou émarginé, un carpelle à style 3-fide, 3-denté ou irrégulièrement laciuié " (1). Daus la plante qui nous occupe nous retrouvons les deux éléments décrits plus haut et qui sont ce que nous avous appelé les pièces postérieures du périgone; mais en ces deux pièces il y a des pièces latérales très larges et celles-ci sont encore enveloppées par deux bractées qui entourent le tout avant l'épanouissement du bouton. Quant aux caractères de l'ovaire et du style. ils correspondent pour notre plante à ceux qui ont été indiqués par les auteurs.

Fig 7 et 8 . - Pièces postérieures du périgone (18/1).

Fig. 9. - Coupe longitudinale antéro-postérieure de l'ovaire (14/1).

Fı. 10. - Ovule isolé (15/1).

Fig. 11. - Diagramme floral.

(1) Cf. Prantl in Engl, et Prantl Naturl. Pflauzenfam. III, 2 p. 84 et Oliv, Fl. (rop). Afr. I p. 45. 
A première vue le $C$. Wildemaniana rappelle le $C$. tenuipes Engl.(1), réeolté au Congo par Pogge et par le regretté professeur Ĺ. Laurent, mais il n'est pas possible de pousser plus avant la eomparaison de ces deux plantes, car la plante du Congo n'est connue qu'avee fleur's mâles, eelle du '/ambize avee fleurs femelles. Cependant, si l'on examine les deux plantes, on saisira bien vite eertaines différenees, par exemple la villosité éparse, mais nette eependant, des tiges de la plante que nous déerivons ici et la longueur des pétioles, qui atteignent de 6 a $8 \mathrm{em}$. elız le $C$. $\ell e-$ nuipes alor's qu'ils mesurent au maximum $3 \mathrm{em}$. de long dans les échantillons, peu nombreux il est vrai, que nous avons pu examiner du C. Wildemaniana.

(1) in Bot. Jahrb. XXVI (1899) p 399. 

PL. Novae HERB. Hort. Thenensis.

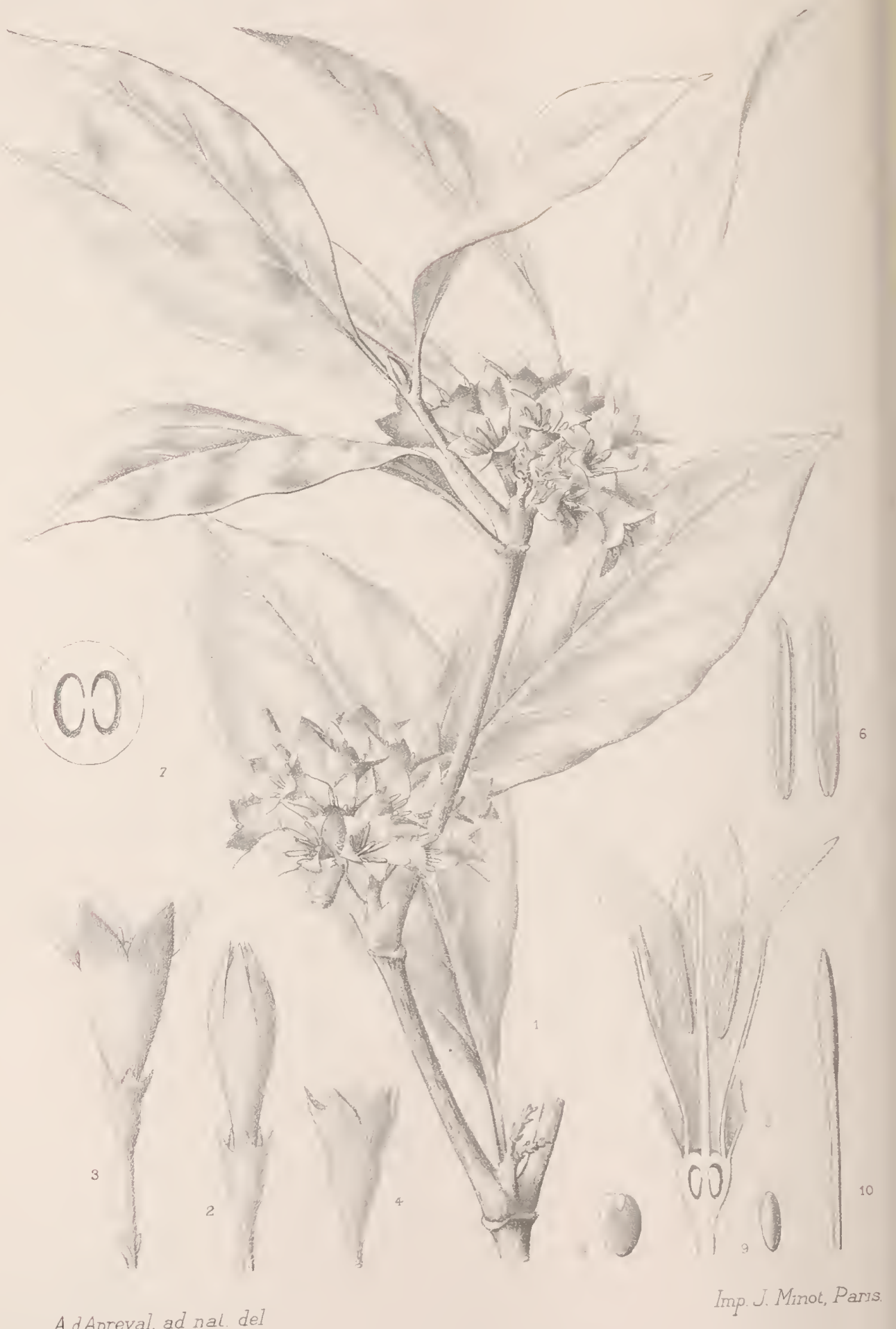

HEINSENIA IU H De Wild 


\title{
Heinsenia Lujæ De Wild. nov. sp.
}

\author{
(RUBIACE E)
}

Heinsenia Lujæ; fruticosa $4.6 \mathrm{~m}$. alta, ramosa, ramis gracilibus glaberrimis; foliis petiolatis oblongo-lanceolatis; attenuato-acuminatis acutissimis basi cuneatis, glabris; stipulis caducissimis, subulatis; panicula terminali dense sed breviter pilosa; floribus albidis pedicellatis, pedicellis pilosis; ovario turbinato ut calyx quinquelobus extus pilosa; corolla extus dense pilosa, intus glabra; stilo corolla non superante.

Arbuste de 4 à $6 \mathrm{~m}$. de haut, rameux, à rameaux assez grêles, à écorce grisâtre, glabres. Feuilles pétiolées, à pétiole de 8 à $15 \mathrm{~mm}$. de long, glabre, canaliculé supérieurement, à limbe oblong-lancéolé, atténué-acuminé, aigu au sommet, cunéiforme à la base, de 8 à $14 \mathrm{~cm}$. de long et 2,2 à $6,5 \mathrm{~cm}$. de large, glabre sur les deux faces, sauf sur les nervures proéminentes de la face inférieure, où se trouvent des poils épars, et à l'aisselle des nervures centrales et latérales où il existe des touffes de poils. Nervures latérales au nombre de 4 à 5 de chaque côté de la nervure médiane, peu marquées au-dessus. Stipules assez rapidement caduques, subulées, devenant plus ou moins

\section{Explication des figures de la planche III.}

Fic. 1. - Rameau florifère (1/1).

FIG. 2. - Bouton $(2,5 / 1)$.

Fig. 3. - Fleur épanouie (2/1).

FIG. 4. - Coupe longitudinale de la fleur (3/1). 
scarieuses et atteignant $4 \mathrm{~mm}$. environ de long. Panicules développées dans la fourehe formée par deux ramifications ou terminant les rameaux, densément mais courtement velues, denses, à plus de 12 fleur's pédicellées, à pédicelle de 4 i $6 \mathrm{~mm}$. de long, velu, à poils apprimés, bractéolé à la base, à bractéoles relues, ciliées. Calice velu, de $4 \grave{a} \mathrm{~mm}$. environ de long, à 5 lobes triangulaires aigus, plus courts que le tube campanulé. Corolle infondibuliforme, blanche, velue extérieurement, glabre intérieurement, de $15 \mathrm{~mm}$. environ de long, à 5 lobes triangulaires, aigus, de $6 \mathrm{~mm}$. environ de long. Etanines insérées dans la gorge. Ovaire soudé au calice, biloculaire, à ovule solitaire dans chaque loge, pendant, surmonté d'un style subcylindrique claviforme, glabre, sillonné.

Hab. - Moçambique : Forêts de Morrumbala, 11 septembre 1900 (Éd. Luja, n. 357).

Observations. - Le genre Hcinsenia a été créé en 1897 par le regretté prof. K. Schumann pour une plante de l'Afrique orientale allemande, découverte par Ileinsen sur les montagnes de Handei, à 900 mètres d'altitude au-dessus du niveau de la mer. Ce genre a été décrit dans le Bot. Jahrbücher du prof. Engler et doit se classer dans le groupe des Albertecie, dans le voisinage immediat du genre Rhabdostigma. Le genre Heinsenia se caractérise surtout par'sa corolle presque campanulée.

Les genres Rhabdostigma Hook. f. et Heinsenia se différencient des genres Aulacocalyx Hook. f. et Psilanthus, qui comme eux sont tous deux endémiques en Ifrique, par la forme de l'extrémité du style; en effet, tandis que chez les deux premiers genres, l'extrémité du style est fusiforme, épaissie et marquée de stries longitudinales, chez Aulacocaly $x$ jasminiflora Hook. f., une espèce assez répandue dans l'Afrique occi-

Fig. 5. - Calice (3/1).

FIG. 6. - Étamines vues de face et de dos (4/1).

F1G. 7. - Coupe transversale schematique de l'ovaire.

Fro. 8 et 9. - Orules vus de dos el de profil (9/1).

Fia. 10. - Style isolé (3/1). 
dentale, le style filiforme est eourtement bilobulé au sommet et clıez les représentants du genre Psilanthus ee style se termine par deux stigmates linéaircs.

La différeneiation des genres Rhabdostigma, à une seule espèec (R. Kirkii Hook. f. de la zone côtière du Moģambique) et Heinsenia se base non seulement, comme nous l'avons dit, sur la forme de la eorolle, mais encore sur la longueur des dents ealieinales; ee qui a fait établir par K. Schumann, dans les Pflanzenfamilien Nachträge zu IV, p. 314 , la elef suivante :

Calice 5 denté; corolle rosacée . . . . Rhabdostigma. Calice profondément 5 -lobé ; corolle campanulée Heinsenia.

Mais nous tenons cependant à faire remarquer que l'on ne peut prendre à la lettre le premier de ces earactères, ear si l'on compare la figure que nous publions ici de l'Heinsenia Lujce avec les échantillons authentiques de $H$. diervilleoides $K$. Schum., on verra qu'il y a entre ces deux espèces une différenee assez notable pour la longueur des lobes du calice; ceux-ci sont beaucoup moins allongés dans la plante recueillie par M. Luja que dans celle recueillie par Heinsen.

Le prof. K. Schumann donnait à son genre nouveau les principaux caractères suivants :

“ Oraire biloeulaire, ovule pendant et solitaire dans ehaque loge. Calice à 5 lobes subulés, séricés intéricurement Corolle campanulée-infondibuliforme, à tube basilaire court. Étamines linéaires, étroites, apiculées, subineluses. Style claviforme, à stigmate côtelé, glabre. Baie globuleuse, couronnée par les lobes ealieinaux."

Caractèr'es, qui, comme on peut le voir en consultant la planche, se retrouvent tous dans notre plante.

Le $H$. Lujar se différencie à première vue par ses fleurs plus fortement velues dans toutes leurs parties, sans compter que la couleur de la corolle est très particulière, rouge vif chez l' $H$. diervilleoides et blanche ehez l'H. Luja.

La deseription spéeifique du regretté prof. K. Sehumann donne pour les inflorescenses : "pannieula laterali n; nous avons inscrit " pannicula terminali ", car il nous a semblé que dans tous les eas les inflorescences terminaient les tiges et se trouvaient ultérieurement siutées dans la fourche formée par le développement des deux bourgeons latéraux axillaires. Le mème fait s'observe d'ailleurs elıez l' $H$. diervilleoides K. Schum., mais l'épaississement du rameau florifêre sous les fleurs est moins aceusé dans les échantillons de Heinsen, que nous avons pu étudier, que dans 
ceux recueillis par M. Luja, dont nous avons pu faire dessiner un fragment.

D'après les renseignements fournis par M. Heinsen, les fleurs du $H$. diervilleoides possèdent une forte odeur rappelant celle des clous de girofle; nous ne possédons aucun renseignement sur l'odeur des fleurs de la plante nouvelle. 


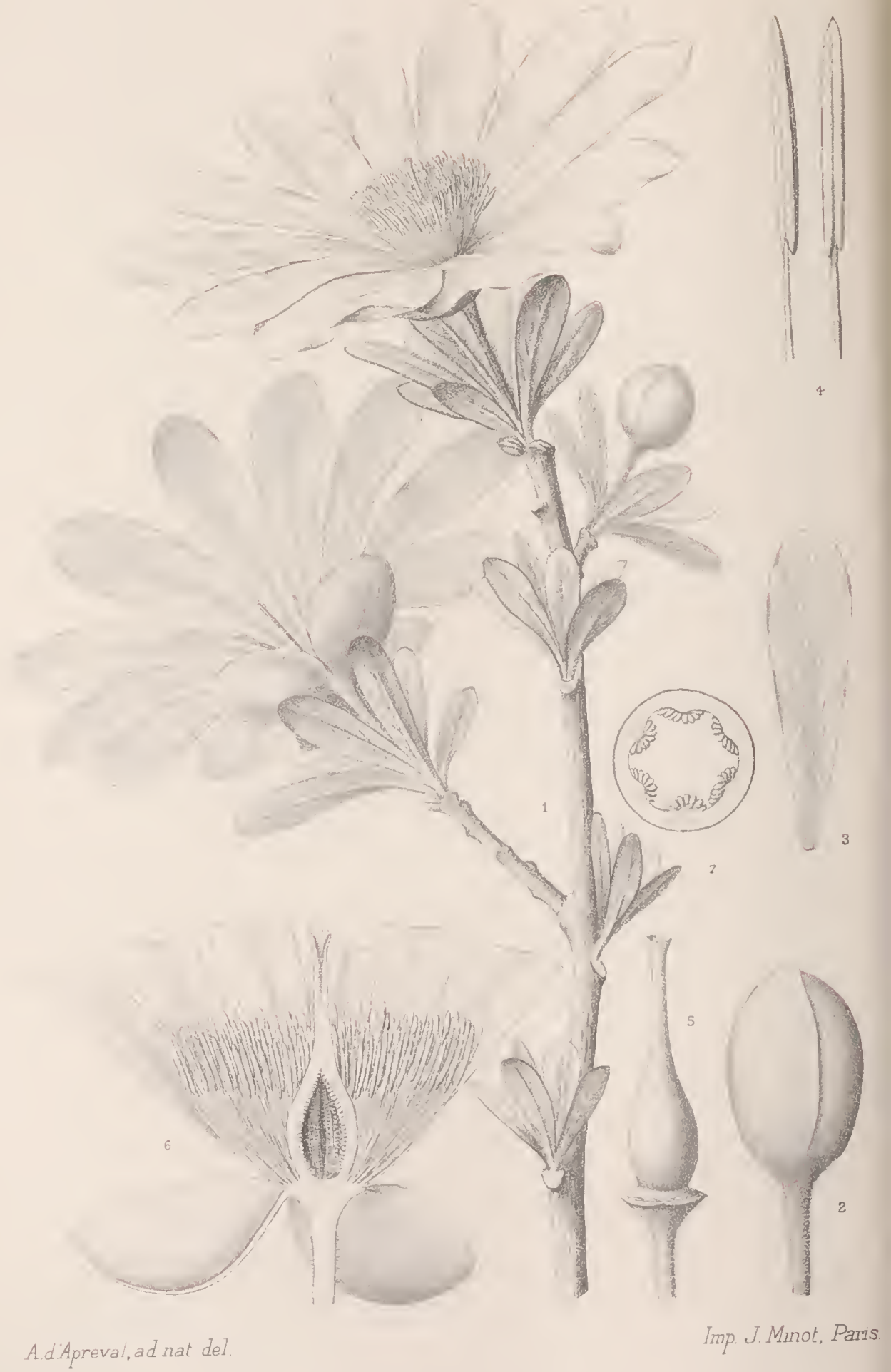

OncoBa Angustipetala De Wil 


\title{
Oncoba angustipetala De Wild. nov. sp.
}

\author{
(FLACOURTIACEA)
}

Oncoba angustipetala; ranulis cinereis, imnovationibus griseopilosis; petiolis $4-5 \mathrm{~mm}$. longis et $1 \mathrm{~mm}$. cire. latis; foliis (adultis?) obovatis-oblanceolatis, dense velutinis, $2-4 \mathrm{~cm}$. longis et $6-10 \mathrm{~mm}$. latis. margine integr'is apice rotundatis; venis 5-6 utrinque, paulo prominulis; inflorescentiis terminalibus, unifloris; perlonculo velutino, tenui, 2-3 cm. longo et $1,5 \mathrm{~mm}$. lato, sepalis extus minutissime puberulis, $15-17 \mathrm{~mm}$. longis et $10 \mathrm{~mm}$. latis, margine scariosis, petalis albidis, $4,5 \mathrm{~cm}$. circ. longis et $8-12 \mathrm{~mm}$. latis; oborato-lanceolatis; filamentis gracilibus, antheris exappendiculatis basi olsseure sulsagittatis, brunneis, $4 \mathrm{~mm}$. longis ; ovario subglabro, ovoideo, non sulcato $4 \mathrm{~mm}$. circ. diam.; stylo quam ovario subxquilongo, conoideo apice obscure denticulato.

Arbre de 6 à $8 \mathrm{~m}$. de haut, à rameaux grisâtres, glables à l'ótat adulte, à rameaux jeunes grisîtres-velus. Pétiole de 4 ì $5 \mathrm{~mm}$. de long et $1 \mathrm{~mm}$. de large, velu; feuilles jeunes ohovales-oblancéolées apparaissant après les fleurs, densément veloutées, de 2 à $4 \mathrm{~cm}$. de long et 6 à $10 \mathrm{~mm}$. le large, à bords entiers, arrondies au sominet, à 5 ou 6 nerrures de chaque côté de la nervure médiane, peu proéminentes sur les deux faces; inflorescences terminant les rameaux ou ramuscules, uniflores, à pédoncule velu, grêle, de 2 à $3 \mathrm{~cm}$.

\section{Explication des figures de la planche IV.}

FIG. 1. - Rameau fleuri (1/1).

Fro. 2. - Bouton (3/1)

Fis. 3. - Coupe longitudinale de la fleur $(2,5 / 1)$. 
de long et $1,5 \mathrm{~mm}$. de large, sépales au nombre de 3 , très courtement pubéruleux extérieurement, de 15 à $17 \mathrm{~mm}$. de long et $10 \mathrm{~mm}$. de large, scarieux sur les bords; pétales hlancs, de $4,5 \mathrm{~cm}$. environ de long et 8 à $12 \mathrm{~mm}$. de large, obovales-lancéolés, obtus, au nombre de 12 environ, étalés. Étamines à filaments grêles, plus longs que les anthères, celles-ci ohscurément subsagittées à la base, brunâtres, de $4 \mathrm{~mm}$. environ de long. Ovaire glabre, ovoïde, non sillonné, de $4 \mathrm{~mm}$. environ de long, surmonté d'un style environ aussi long. subconique et terminé par un entonnoir peu développé, obscurément denticulé.

Hab. - Moçambique : Forets de Morrumbala, novembre 1900 (Éd. Luja, n. 395).

Obscrvations. - Cet Oncoba appartient au groupe d'especes earaetérisćes d'après M. Oliver(Fl. trop. If I I p. $11^{\prime} 4^{\prime}$ par les fleurs terminales ou axillaires, solitaires ou fascieulées, mais non en raè̀mes. Par ses feuilles pubescentes à l'état jeune il rappelle les O. Petersiana Oliv., O. Tettensis Oliv., O. Kraussiana Planch et O.brachyanthera Oliv. Si l'on suit la elassitieation adoptée pour les espèces de ee genre, dans la Revision des Flacourtiacen publiée rar M. le professeur O. Warburg dans les Pflanzenfamilien IV, 2 p. 17, la plante que nous figurous ici doit se ranger dans la seetion Euoncoba Warb. earactérisée par ses fruits incrmes, ses feuilles relativement rérluites, glabres ou velues, privées l'éeailles. Dans rette seetion M. le professeur O. Warburg a délimité deux subdivisions: Acanthoncoba à tiges épineuses et feuilles largement dentées et L.ysoncoba à feuilles entières, velues au moins pendant le jeune âge. C'est naturellement dans cette deuxième subdivision que nous devons ehercher à elasser l' $O$. angustipetala. Dans ce groupele professeur O. Warburg range l'O. macrophylla (Kl.) Warb. que l'on rencontre du Mogambique au Natal et qui possède des lobes stigmatiques allongésaigus, l'O. rettensis (Kl.) Oliv. que l'on rencontre dans la région du Zambèze et du Mogambique et qui, Jui, rappelle notre plante par ses feuilles

Fig. 4. - Pétale (1/1).

FIG. 5. - Étamines (9/1).

Fir. 6. - Ovaire et style $(2,5 / 1)$.

Fig. 7. - Coupe transversale de l'ovaire (5/1). 
velues et ses lobes stigmatiques courts, mais tandis que cliez l'O. teltensis les pétales sont velus ou au moins eiliés, ehez la plante nouvelle ils sont totalement glabres; la fleur de eette dernicre est d'ailleur's beaucoup plus grande; en effet, clle ne dépasse guère $6 \mathrm{em}$. de diamètre chez l' $O$. letlensis. Dans la même subdivision nous trouvons encore l'O. Stuhlmanni Gï̈rke (in. Engl. Bot. Jahrb. XVIII p. 161) dont les feuilles adultes mesurent 7 ì 8 cin. de long, mais dont les pétales mesurent de 25 à $30 \mathrm{~mm}$. de loug sur 12 à $15 \mathrm{~mm}$. de large, e'cst-à-dire qu'ils sont relittivement beaucoup plus larges que ceux de notre espèce nouvclle. M. Gürke ue dit point si les pétales sont velus, et ne fournit aucun renseignement sur l'extrémité du style. Quant à l'O. Kraussirma Plinelı., figuré dans les Natal Plants de MM. Medley Wood et M. S. Evans, I 11. 72, il possède des feuilles aiguës, des fleur's plus réduites et un stigrmate à 5 ou 6 rayons, d'apres M. le professeur Warburg, à 3 lobules irréguliers, d'apres les figures 2 et 3 de lit planche 722 des "Nital Plants "; en outre, les pétales de l'O. Lraussiana sont ciliés sur les bords ct eeux de l'O. angustipetala totalement glabres. Ces caractères nous ont paru suffisants pour l'établissement d'une espèce nouvelle qui paraît se rapprocher le plus de l'Oncoba Kraussiana.

C'est une des belles plantes de la r'égion, où elle se fait remarquer' comme toutes ses congénères par ses grandes fleurs apparaissant avint les feuilles. 




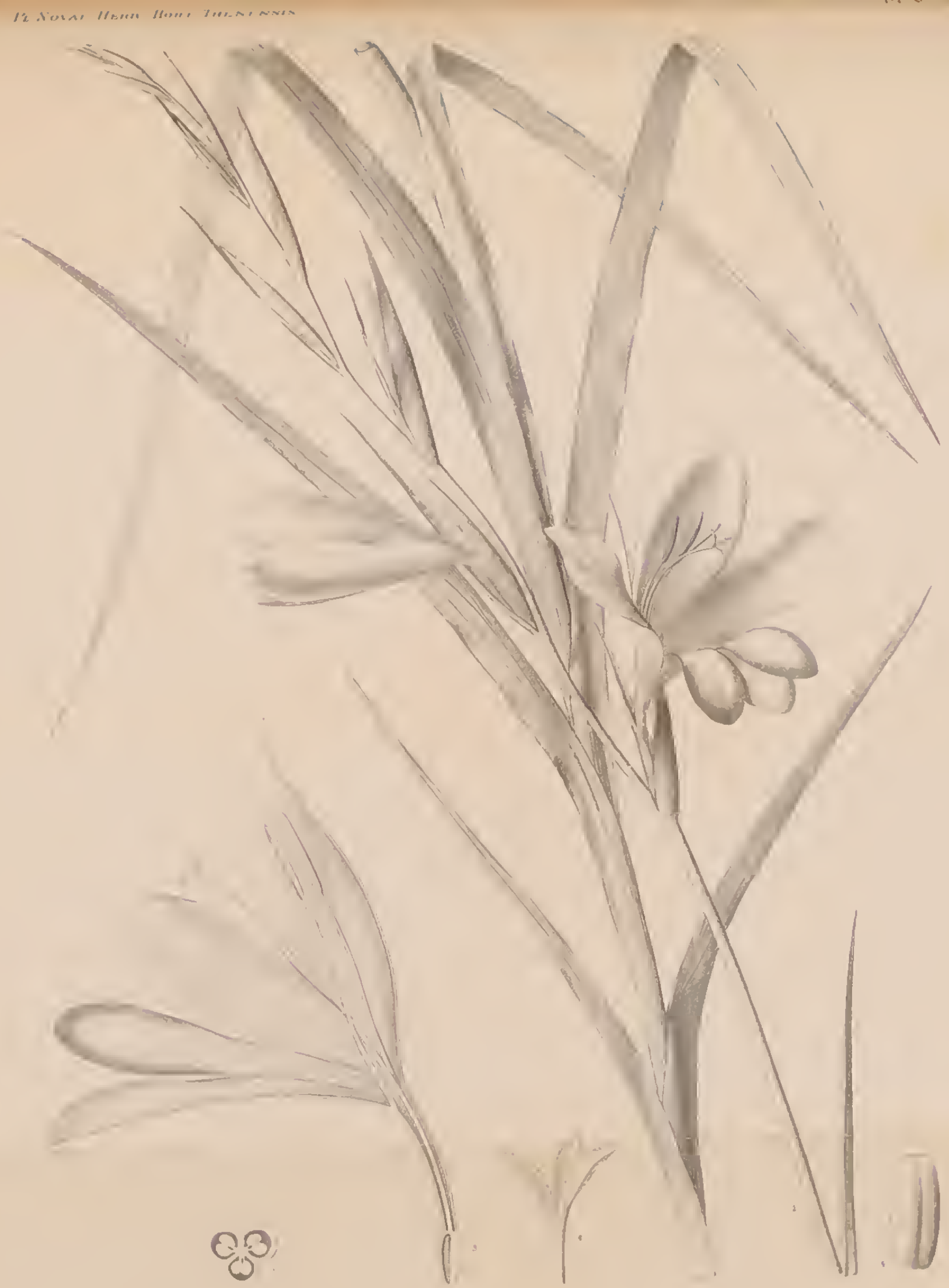




\section{Gladiolus morrumbalaensis Me Hill. $\| \cdots$. s..}

(IRII)ACE.E)

Gladiolus morrumbalaensis ; elatus, glaberrimus, foliis lineariensiformibus striatis, spica 20-30 cm. longa, laxiflora, floribus distantibus, spathis lanceolatis-acutis, tubum perianthii superantibus; lobis obovatis, 3 -exterioribus angustioribus, interiorum 2 lateralibus supremus pallide purpureis et plaga mediani aurea pictis, stigmate breviter trifido, interioribus late obovatis, purpureo-violaceis, basi flavis.

Plante dressée, atteignant $75 \mathrm{~cm}$. de haut, assez forte, glabre. Feuilles ensiformes, atténuées en une pointe aiguë, et mesurant parfois plus de $50 \mathrm{~cm}$. de long et 9 a $15 \mathrm{~mm}$. de large, à nervures proéminentes, au nombre de 2 à 3 de chaque còté de la nervure médiane, s'anastomosant à la base avec celle-ci à différentes hauteurs. Épi florifère de 20 à $30 \mathrm{~cm}$. de long, laxiflore, à 6 à 10 fleurs penchées, distantes de 4 à $5 \mathrm{~cm}$. Bractées au nombre de deux, entourant la base de la fleur; bractée extérieure lancéolée-aiguë, atteignant $7 \mathrm{~cm}$. de long et $6 \mathrm{~mm}$. de large, dépassant le tube du périanthe, bractée extérieure plus courte, de même forme, de $5 \mathrm{~cm}$. de long sur 4 à $5 \mathrm{~mm}$. de large. Fleurs de 7,5 à $8,5 \mathrm{~cm}$. de long, subcampanulées, à lobes recourbés. Périanthe à

\section{Explication des figures de la planche V.}

Fro. 1. - Base de la tige florifere (1/1).

Fı. 2. - Extrémité du rameau florifère, avec fleur épauouie et boutons à divers stades $(3 / 1)$.

Fro. 3. - Coupe longitudinale de la fleur $(1,5 / 1)$. 
tube violaré, les 3 lobes supérieu's assez largement obovales, violacés, à partie médiane jaunâtre et à hase jaune, atteignant $2,7 \mathrm{~cm}$. de diametre dans leur partie médiane, les 3 inférieurs plus étroits, onguiculés, atteignant $15 \mathrm{~mm}$. de diamètre dans leur partie médiane, les deux latéraux à bord violacé et à zone médiane jaune. Anthères linéaires, de $\mathrm{Jl}$ à $14 \mathrm{~mm}$. de long, style aussi long que les anthères, à 3 lobes ubovales, glanduleux sur les bords.

\section{Hab. - Moçambique : Morrumbala. décembre 1900 (Éd Luja, n. 393).}

Obser'vations. - La jolie espèce que nous venons de figur'er' et de décrire parait se rapprocher du $G$. Papilio Hook., décrit et figuré dans le Bot. Mag. tab. 5565, mais cette dernière espèce existe dans les Colonies du Cap, de l'Orange et du Transraal seulement; elle n'est donc pas, comme celle que nous figurons ici, franchement tropicale. La distinction des espèces de ce genre n'est guère facile, et leur classification dans un des quatre sous-genres proposés par M. Balier n'est pas aisée; en effet, si l'on compar'e entre eux les caractères de ces quatre groupes, que nous rappelons ci-dessous, et si l'on examine ensuite un certain nombre des espèces qu'il faut intercaler', on s'apercerra bien rite qu'il y a des transitions nombreuses (cf. Handbook of Irideae, p. 198).

Sous-genre Eugladiolus. - Tube du périanthe en entonnoir; segments non distinctement onguiculés.

Sous-genre Hebea. - Tube du périanthe court, à segments distinctement onguiculés. Spathes larges.

Sous-genre Schweiggera. - Fleur's petites, à segments distinctement onguiculés. Spathes petites.

Sous-genre Homoglossum. - Périanthe à tube grêle, allongé, brusquement élargi vers le milieu, à segments subaigus.

C'est certes dans le premier de ces sous-genres qu'il faudra classer le nouveau glaïeul, bien qu'il $y$ ait lieu de faire r'emarquer que les lobes inférieur's du périanthe sont plus ou moins rétrécis rers la base

Fro. 4. - Ovaire (3/1).

Fıg. 5. - Ovaire en coupe transversale, coupe schématisée.

Fır. 6. - Extrémitè du style avec les 3 stigınates foliacés $(5 / 1)$ 
et presque onguiculés. Dans sa monograplie des trideae M. Baker range 109 espeecs dans le sous-genre: 15 appartiement à la flore de l'Europ'e et de l'Asie, toutes les autres à celle de l'Afrique, loealisées au ('ap ou en Afrique tropicale. Pour différ'eneier ees 94 espèces, M. Baker lropose de se baser sur la forme des feuilles; eelles-ei peuvent ĉtre subeylindriques, linéaires on ensifor'mes, nous ne pouvons naturellement classer notre espèce dans le groupement à feuilles linéaires ou subeylindriques, puisque les feuilles mesurent jusque $12 \mathrm{~mm}$. de diamètre à la base; elles sont d'ailleurs netrement ensiformes, mais nous nous trourons encore en frésence de 38 espèces appartenant à cette dernière sublivision. Ces esprees sont :

G. Leichlini Bäker, Gi. H.lliolii Baker, G. crassifolius Baker, (r. sericeo villosus Hook., G. Ludwigii Papp., G. ochrolencos Baker, G. Eckloni Lelım., (r. pur purco-amalus Hock. f., G. Papilio Hook., G. Rehmanni Baker, G. hirsulus Jaẹ!., G. blandus Ait., G. salmoneus Baker, Gi. scuphochlamys Baker, (i. floribundus Jaeq., G. cppositiflomus Herb., G. Milleri Ker., G. undulatus Jaeq., G. Adlami Baker. $G$. Macouani Baker, G. splendens Baker, G. cardinalis Curt., G. cruentus Moore, G. dracocephalus Hook., G. psitlacinus Hook., G. Tysuni Baker, G. Saundersi Hook., G. aurantiacus Klatt, toutes du sud de l'A frique et $G$. Buettneri Pax. ('Tngo), G. Lenguelensis Balier (Angola), G. spicatus Klatt (Guinéc), G. multiflorus Balier (Angola), $G$. andongensis Welw. (Angola), G. decoratus Baker (Moramballa, Shire), G. prinnulinus Bakel (Afrique tropicale orientale, Usagara), $G$. corneus Oliv. ('Tanganika), G. Lirkii Baker (Zanzibar).

Cette liste devrait être ccinplétée par' un assez grand nombre d'espèces publiées ultérieurement au travail monographique de N. Baker, nous ne croyons pas deroir reproduire ici toute eette nomenelature; nous avons tenu à donner cette énumération afin de faire roir que le nombre des espèees de l'. If rique tropieale est relativement réduit et qu'une seule espèee de cette longue liste se reneontre dans la région d'où provient notre plante. Dans la Flora of tropical Africa VII de M. Thiselton-Drer. direeteur des Jardins royaux de Kew, M. Baker a repris l'étude des Gladiolus africains et a publié un tábleau analytique sur lequel nous allons nous baser pour essayer de donner les affinités de notre espèce (cf. loe. cit. p. 360 et suiv.) Il elasse les espèces du sous-genre Eugladiolus en 4 groupes :

Feuilles étroites; fleurs pretites.

Feuilles étroites; fleurs grandes.

Feuilles relativement larges; fleurs petites.

Feuilles relativement larges; fleurs grandes. 
Feuilles étroites signifie au maximum $12 \mathrm{~mm}$. de diamètre; notre plante possédant des feuilles pouvant atteindre $18 \mathrm{~mm}$. de diamètre et des lleur's grandes, c'est indiscutahlement dans le dernier de ces groupes qu'il faudra la ranger. Pour aller plus avant dans la différenciation. M. Baker classe d'un côté dans cette subdivision les espèces dont le segment supérieur de la corolle est cucullé, et d'un autre côté celles dont le segment n'est pas cucullé. La figure 2 de notre planche ne peut laisser de doute, le segment supérieur du périanthe du $G$. morrumbalaensis n'est pas cucullé.

Il n'existe dans ce sous-groupement que 3 espèces se différenciant comme suit :

Segment supérieur non cucullé.

perianthe rose.

Périanthe d'un rouge foncé.

Périanthe de 36 à $48 \mathrm{~mm}$. de long; valre extérieure de la spathe de 24 à $30 \mathrm{~mm}$. de long.

Périanthe de $7,2 \mathrm{~cm}$. de long; valve extérieure de la spathe de 36 à $48 \mathrm{~mm}$. de long . .
G. brachyandrus Baker (Nyasaland, Shire).

\section{G. benguellensis Baker} (vide supra).

\section{G. decoratus Balier}

(vide supr'a).

Cette dernière espèce est la plus voisine de notre plante; elle habite d'ailleurs la même région. Mais, dans la plante recueillie par' M. Luja, les fleurs sont plus développées et les bractées florales sont également plus longues, comme on pent le voir en consultant la description ci-dessus; en outre, tandis que chez le $G$. decoratus, introduit dans la culture en 1887 , les pétales supérieur's mesurent $12 \mathrm{~mm}$. envirou de diamètre, dans notre plante ils mesurent plus de $2 \mathrm{~cm}$.

La couleur “ bright red" du G. decoratus diffère peut-être également un peu de la couleur violacée des fleur's de notre plante, il est rrai que nous connaissons uniquement la plante recueillie par Luja, d'après des échantillons secs dont la couleur a pu être altérée. 




\section{PLANT $\nRightarrow$ NOV $Æ$ VEL MINUS COGNITE}

EX HERBARIO HOR'TI THENENSIS

\section{PLANTES NOUVELLES OU PEU CONNUES}

CONTENUES DANS L'HERBIER

$\mathrm{DE}$

\section{L'HORTUS I'HENENSIS}

\section{AVEC LES DESCRIPTIONS OU ANNOTATIONS}

DE

M. Ém. DE WILDEMAN

DOCTEUR EN SCILNCES

CONSERYATEUR AU JARDIN BOTANIQUE DE L'ÉTAT

\section{Deuxième livraison.}

(JUIN 1904)

VEUVE MONNOM

IMPRIMFUR ET FDITEUR

32, RUE DE L'INDUSTRIE, 32

BRUXELLES

Se vend exclusivement à l'Administration des biens et affaires de M. van den Bossche, 5, Grande Montagne, à Tirlemont. 



\section{DEUXIËME LIVRAISON}

Lit seconde livraison des Plante Nove est exclusivement consacrée à quelques espèces de Cypéracées sudafricaines, les unes non encore décrites, les autres déj:i publiées dans le dernier volume de la Flora Capensis.

Les matériaux d'analyse se trouvent dans les Plante Schlechteriance Austro-Aficance, dont le Musée royal de botanique de Berlin a bien voulu ine céder un grand nombre de doubles.

Ces matériaux n'avaient encore pu être déterminés jusqu'à ce jour. Nous avons prié M. C. B. Clarke de nous aidler de ses lumières et le savant anglais, dont la haute compétence en la matière est reconnue du monde entier, a daigné répondre à notre demande avec une bienveillance et un empressement dont nous lui sommes très reconnaissants, - en nous permettant en outre de publier dans ce recueil les plantes non décrites dont il nous a communiqué les diagnoses. Qu'il veuille bien trouver ici l'expression de toute notre gratitude.

Nous pouvons ainsi présenter luit espèces distinctes appartenant à des genres diffërents et nous espérons que cette contribution nouvelle à l'étude des Cypéracées, par l'intérêt qu'elle présente comme par la fidélité des analyses qui sont quelque peu de nature à rebuter le dessinateur, rencontrera l'approbation des spécialistes.

L. B. 
La grande famille des Cyperacece comprend plus de 3,300 espèces répandues dans les régions sèches et humides du globe entier. Dans une famille aussi vaste il existe naturellement un grand nombre de variations qui rendent l'étude des espèces très difficile; en outre, la petitesse des organes fait de ces plantes un groupe délaissé par li plupart des botanistes et fréquemment mênı les voyageurs ne tiennent pas à les récolter.

Sans les travaux si remarquables de M. C. B. Clarlie, l'on se trouverait encore aujourd'hui dans un chaos indébrouillable. Nous sommes heureux d'avoir pu, pour l'étude des quelques espèces choisies par nous pour figurer dans cette publication, nous en rapporter à ces travaux.

1)'après le savant anglais, les genres Scirpus, Eleocharis et Hicinic appartiennent à la tribu des Scirpere; les genres Carpha et T'etraria à celle des Schenece. Les deux tribus se rangent l'une et l'autre dans le sous-ordre des ScirpoSchonew auquel M. Clarke oppose le sous-ordre des Caricex.

La classification de l'École de Berlin formulée par M. Pax dans le septième volume des Botanische Jahrl. (1886) et dans les Pflanzenfam. II 2 (1887), admise également par M. le Prof. Engler dans son "Syllabus ", est quelque peu différente. Nous la rappelons comme suit: I. Sous-famille des Sciryoidece, comprenant les tribus des Hypolytrea et Scirpece; II. Sous-famille des Caricoidece, comprenant les tribus des Rhynchosporea, Gahniew, Bisbrekelerice, Scleriece et Curicece. Dans cette classification, les genres Carpha, Ficinia, Scirpus et Eleocharis appartiennent tous les quatre à la tribu des scirpece et le genre Tetraria à la tribu des Gahniece. 


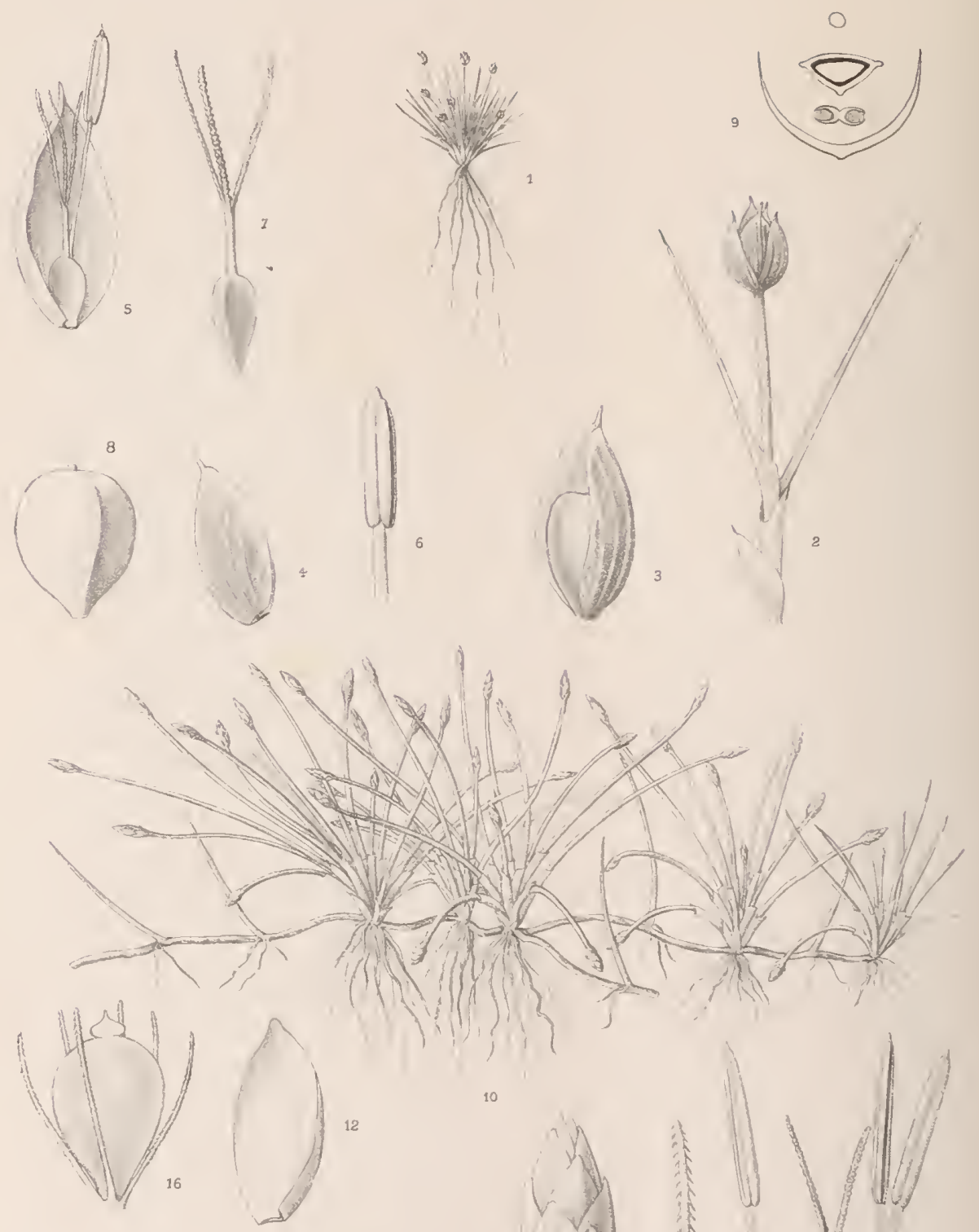

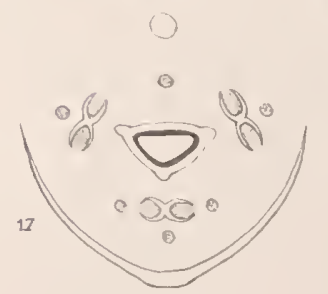

Ad'Apreval ad nat de?

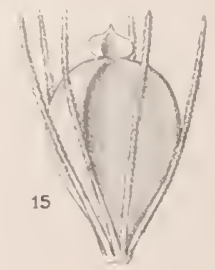

10
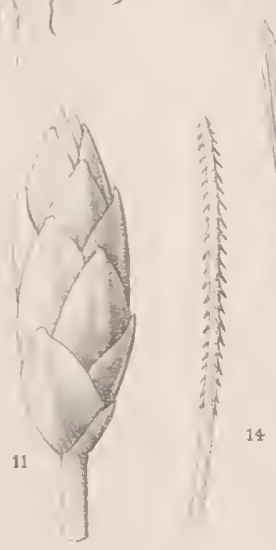

Imp.J.Mino: Pans

SCIRPUS IIEMLUNCIALIS C B CLIKe. (Fig.1-9)

ELEOCHARIS SCHLECHTERI C.B Iarke (Fig.10-17) 


\section{Scirpus hemiuncialis C.B. Clarke nor. sp.}

Scirpus hemiuncialis; culmis foliisque 1/2-2/3 uncialibus, foliis apice submucronatis, vaginis dilatatis albis, spica 1 ebracteata, $1 \mathrm{~mm}$. in diam., globosa, proventu ellipsoidea, $11 / 2 \mathrm{~m}$. longa, pauciflora; glumis quadrato-ovatis, purpureo-rubris ; stylo 3 -fido; nuco minutissima cum $1 / 2$ parte glumie vix xquilonga, ellipsoidea, trigona, levibrunea (Sc. cernua Vahl affinis).

Petite plante glabre, formant des touffes feuillées, de 12 i $16 \mathrm{~mm}$. de haut. Feuilles peu nombreuses, engainantes à la base, aiguës submucronées au sommet, à gaine plus ou moins dilatée, blanchâtre, environ aussi longue que la tige, dépassant parfois légèrement la tige florifère. Épillet solitaire, privé de bractées basilaires, de $1 \mathrm{~mm}$. environ de diamètre, sul)globuleux ou ellipsö̈de, atteignant parfois 1, ¿ mm. de long, pauciflore; glumes d'un pourpre rougeâtre, quadrangulaires-ovales, striées, mucronées. Étamine solitaire, à filet grêle, presque aussi long que le style et les stigmates, à anthère dépassant les glumes, légèrement apiculée au sommet. Ovaire obovoïde à style

\section{Explication des figures de la planche VI}

Fig. 1. - Plante entière (1/1).

FIG. 2. - Tige florifère avec feuilles engainantes (6/1).

FıG. 3. - Glume renfermant un akène (25/1).

Fig. 4 - Glume vue de profil (20/1).

Fig. 5. - Fleur entourée de sa glume (30/1).

FIG. 6. - Anthère (40/1).

Fıa. 7. - Ovaire, style et stigmates (40/1). 
environ aussi long que l'ovaire ; stigmates au nombre de 3 , plus longs que le style. Aliène environ de la longueur de la moitié de la glume ou aussi long, elliptique, trigone, lisse, brunâtre.

Hab. - Afrique australe : Région austro-occidentale, Zout Rivier, 450 pieds, 13 juillet 1896 (R. Schlechter, n. 8118 ).

Observations. - Le genre Scirpus renferme plus de 130 espèces dont plusieurs sont largement répandues sur le globe; il en existe des représentants dans presque toutes les contrées et sous tous les climats.

La curieuse petite espèce que nous figurons ici est roisine du $S$. cernuus Vahl, une espèce décrite sous divers noms et même figurće par Rottboel sous le nom de $S$. setaceus dans ses Descriptions et Iconcs 47 , tab. 15, fig. 4, et très répandue dans les r'égions chaudes et tempérées. Dans les espèces du sud de l'Afrique, M. C. B. Clarke considère 5 sous-genres, dont la distinction n'est pas des plus aisćes; ces sousgenres sont: Fluitantes, Isolepis, Euscirpus, Micrantha, Pscudoschoenus. Ce dernier' sous-genre est très reconnaissable par ses épillets réunis cn une panicule ramifiée allongée et par la prósence de soies hypogvues; le sous-genre Micrantha est caractérisé par l'absence presque totale de style. Chez les Euscirpus il existe fréquemment des soies hyporynes. Quant aux Fluitantes et aux Isolepis, leur diffćrenciation repose sur les caractères suivants :

Tiges avec ncuuls rortant des feuilles o' privés de feuilles . . . . . . . . . . Fluitantes.

Tiges avec feuilles basilaires seules ou aplyylles. . Isolepis.

Dans ce dernier groupe, auquel appartient indiscutablement la plante que nous figurons, M. C. B. Clarke considère 4 groupements, le premier à style bifide, les 3 autres à style trifide, mais les espèces du quatrième groupe sont caractérisées par des ćpillets en ombelle, les deux autres ont des épillets solitaircs ou capitulés. mais dans le groupe 3 les akènes sont lisses tandis que dans le groupe 2 ils sont trabéculés; nous rangeons notre plante dans lo groupe 3 en nous basant sur la clef proposée par II. C. B. Clarke; nous dirons done pour differencier entre elles les espèces voisines du $S$. hemiuncialis :

Fig. 8, - Akène mûr (29/1).

FIg. 9. - Schéma de la fleur. 
Tiges vertes, sétacées.

Plante de 5 à $16 \mathrm{~cm}$. de haut. . . . .

Plante de 12 à $16 \mathrm{~mm}$. de haut. . . . . S. hemiuncialis C. B. Clarke.

Tiges noires, capillaires . . . S. subprolifer Boeck.

'Tiges noires, épaisses. . . . S. rivularis Boeck. 



\section{Eleocharis Schlechteri C. B. Clarlie.}

in This.-Dyer Fl. Capensis VII (1900) p. 758.

T'iges de 12 à $40 \mathrm{~mm}$. de haut réunies en touffes rigides le long d'un rhizome. Gaine foliaire supérieure assez ferme, entière, courtement oblique au sommet. Épillet terminal de $6 \mathrm{~mm}$. environ de long sur $2-3 \mathrm{~mm}$. de large, contenant de 8 à 16 akènes, lancéolé, entouré de glumes elliptiques, carénées, subapiculees, obtuses, scarieuses su les bords. Soies hypogynes d'un brun cannelle, au nombre de 6 à 7 , dépassant l'ovaire, scabres, à poils recourbés. Étamines à filet plus long que les soies hypogyres et que l'ovaire, à anthères obtuses, courtement apiculées, plus courtes que les filets, dépassant les stigmates. Ovaire obovale assez nettement trigone dans le jeune âge, à style fortement renflé à la base, divisé en deux stigmates; akène obovoïde, biconvexe, rétréci vers le sommet, devenant noir, lisse.

Hab. - Afrique australe : Région austro-occidentale. - Caledon division : Onrust Rivier (R. Schlechter, n. 9484). - Vogelgat, 11 avril 1897 (R. Schlechter, n. 10410).

\section{Explication des figures de la planche VI.}

Fici. 10. - Stolon avec toufles de liges (1/1).

Fig. 11. - Épi Horifere (8/1).

FIG. 12 - Glume oblusément mucronée (121).

Fig. 13. - Fleur hermaphrodite (20/1).

Fici. 14. - Soie hypogyne (40/1).

Fic. 15-16. - Akènes à maturité (15/1).

FIa. 17. - Schéma de la fleud. 
Observations. - C'est un échantillon de la demière de ces deux localités non signalée dans la F'lora Capensis qui a servi pour faire les analyses de la planche ci-contre. Ia plante que nous arons figurée diffère peut-être un peu du type qui a servi à M. C. B. Clarke pour ćtablir son espèce; en effet, on trouve assez souvent dans les épillets de la plante figurée des akines trigones présentant sur leur face dorsale une carène assez obtuse, mais néanmoins bien visible.

Le grenre Eleocharis créé par R. Brown est représenté dans la pluprart des régions du globe, mais son grand centre de dispersion est l'Amérique, oì l'on rencontre plus de quatre-ringts espèces sur les cent vingt environ yui constituent le gremre. 'Trois espèces seulement existaient en Afrique ; la seule espece endémique actuellement est la plante nouvelle rencontrée lar M. R. Schlechter, toutes les autres espèces existent soit en Asie, cn Australie, à Madagascar ou dans d'autres régions tempérées.

Les especes africaines appartiemnent à 3 sections ou sous-genres caractérisés comme suit :

Section I. - Limnochlon Nees : T'iges vigoureuses. Fipillet à peine plus large que la tige; glumes suln'igides . . . . E. fistulosa Link. (Afrique australe, Afrique tropicale, Asie, Australie)

Section II. - Eleogenus Nees : Epillet plus large que la tige; glumes membraneuscs. Style bifide.

Section III. - Eneleocharis C. B. Clarke : lipillet plus large que lia tige; Glumes membraneuses. Style trifide. . . . . E. liniosa Schulte.

(Afrique australe, Madagascar).

C'cst dans la seconde section que se range l' $E$. Schlechleri, à côté de $l^{*} E$. palustris R. Br.; on diffërencie facilement ces leux espèces pal leurs mensurations.

'Tiges de 9 à $48 \mathrm{~cm}$. de long; épillets de 8-16 mm. de long. . . E. palustris R. Br. (Dans les régions tempérées du globe, plus rare dans les régions tropicales). Tiges de 12 à $40 \mathrm{~mm}$. de long'; épillet de $6 \mathrm{~mm}$. environ de long. . . . . . . E. Schlechteri C. B. Clarke. 



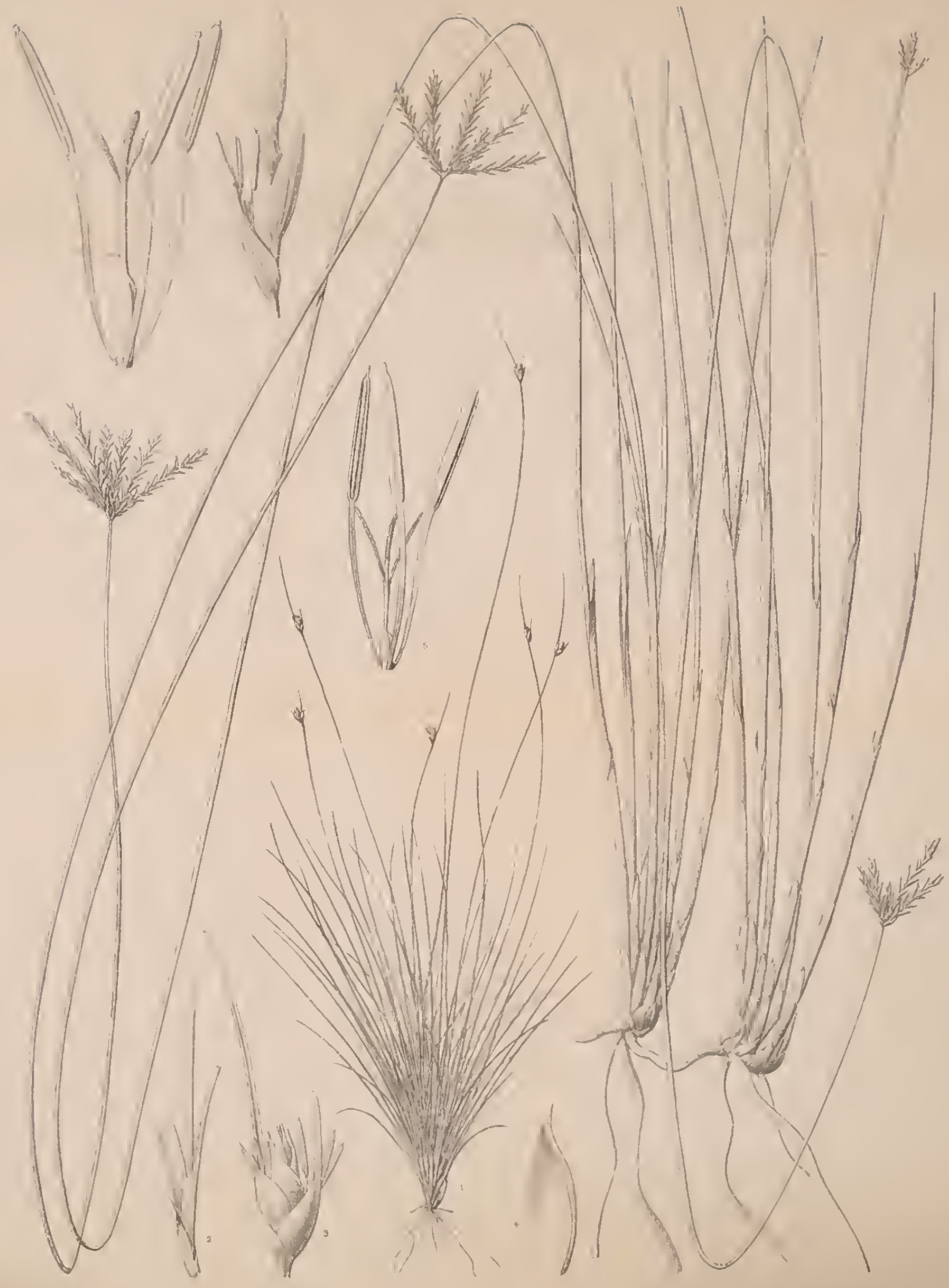




\section{Ficinia mucronata C. B. Clarlie sp. nov.}

Ficinia mucronata; culmis foliisque setaceis, $5-20 \mathrm{~cm}$. longis, culmi floriferi vagina summa folio $4 \mathrm{~cm}$. longo ; bracteis 2 , inferiore setacex, 5-25 mm. longa; spicula 1, ovoidea, juvenili vix $5 \mathrm{~mm}$. in diam.; glumis nucigeris ovatis, apice obtusis, scariosis dentatis, carina in mucronem rigidiorem linearem rubrum excurrente (sp). $F$. pusilla C.B. Clarke forsam affinis).

Plante glabre, à racines fibreuses, formant de petites touffes atteignant une vingtaine de centimètres de hauteur. Feuilles toutes basilaires n'atteignant parfois que la moitié de la longueur des tiges florifères, sétacées, engainantes à la base, aiguës au sommet, à gaines scarieuses. Tige florifère privée de feuilles; épi terminal entouré par deux bractées dressées dépassant l'épillet, élargies à la base, terminées par un long mucron cilié, scabre sur les bords. Glumes fructifères naviculiformes, ovales obtuses, d'un brun rougeâtre, scarieuses sur les bords, carénées, à carène terminée en un mucron rigide, linéaire, rougeâtre. Étamines à filet légèrement aplati, aussi long que l'ovaire et le style; anthère terminée par un mucron obtus. Ovaire

\section{Explication des figures de la planche VII.}

Ficinia mucronata. - Fig. 1 à 5.

Fro. 1. - Plante entière (1/2).

FIG. 2. - Base d'une tige avec feuilles engainantes (5/1).

Fı. 3. - Capitule terminal montraut les bractéss enveloppant les épillets (8/1).

Fı. 4. - Glume isalée, montrant le mucron terminant la carène (15/1).

FIg. 5. - Fleur complète (20/1). 
plus court que le style, cclui-ci linéaire, à 3 stigmates aussi longs que lui.

Hab. - Afrique australe. Région austro-occidentale ; Ezelbank, 4,500 pieds (R. Schlechter, n. 8816, 2 septembre 1896).

\section{Ficinia distans C. B. Clarke sp. nov.}

Ficinia distans; culmis longis, gracilibus, enodiis monocephalis, imâ basi a raginis tenuibus rubris intectis; spiculis 5-9, digitatis, usque ad $35 \mathrm{~mm}$. longis, anguste linearibus; glumis distichis, distantibus, non imbricatis glabra. Culmi $8-9 \mathrm{~cm}$. longi, basi usque ad $10-15 \mathrm{~cm}$. a vaginus intecti; ragine summe pan libera usque all $2 \mathrm{~cm}$. longa, tenuis, obtusa, scarioso-rubro-brunea. Inflorescentia subterminalis, ebracteata. Speculæ $3 \mathrm{~mm}$. latr, usque ad 22 floræ. Glumse inter se $2 \mathrm{~mm}$. distantes, suberectre, elliptico oblongx, obtusie, compressie, castaneo-brunce. Nux majuscula, oblongo-ellipsoidea, trigona, levis, apice obtusa. - Species ingenere Ilemichlaena Sclırader, militans.

Plante à tiges allongées, grêles, glabres, privées de nouds, de 20-70 cm. de long, munies de gaines brunàtres, celles du sommet possédant à leur extrémité une portion libre atteignant $2 \mathrm{~cm}$. de long, obtuse, scarieuse, d'un rouge-brun comme le reste de la gaine. Tiges à un seul capitule terminal, privé de bractées, à épillets digités. Épillets de $3 \mathrm{~mm}$. de diamètre environ, comportant parfois 22 fleurs. Glumes distantes entre elles de 2 mm., subérigées, elliptiques, oblongues, olstuses, comprimées, d'un brun assez foncé, scarieuses sur le bord. Etamines au nombre de trois, à filet allongé plus ou

Ficinia distans. - Fig. 6 à 8.

FIv. 6. - Fragment du thizome avec 2 touffes de tiges (1/1).

Fig. 7. - Fragment d'une des ramifications de l'inflorescence $(8 / 1)$.

FIG. 8. - Fleur isolèe (17/1). 
moins aplati, aussi long que la glume, à anthère plus courte que le filet, apiculée au sommet, à apicule subglobuleux. Ovaire triangulaire, glabre, oblong-elliptique, lisse, obtus au sommet, terminé par un style environ aussi long que l'ovaire et par trois stigmates subétalés.

\footnotetext{
Hab. - Afrique australe - Région austro-occidentale : Vogelgat, 200 pieds, 2 décembre 1895 (R. Schlechter).
}

Obscrvations. - Le $F$. mucronata est, d'après les indications de M. Clarke, une espèce voisine du $F$. pusilla C. B. Clarke, qui a été élevè au rang d'espèce par le monographe anglais après aroir été considéré par lui comme une rarieté capillaris du $F$. Bergiana. Cetto dernière espece est connue de la région côtière et de la région oceidentale. Le caractère différentiel principal est celui sur lequel est fondé le nom spécifique; en effet, eliez le $r$. pusilla, les glunes sont ovales obtuses et non mucronées, comme elles le sont si nettement dans la plante réeoltée à Ezelbank par M. R. Schlechter.

Quant au $F$. distans, il appartient, d'après la diagnose fournie par M. Clarke, au petit groupe "Hemichlacna" eonsidéré comme genre par Schrader. En Afrique australe ee sous-genre ne comportait que 3 espèces; l'espèce nouvelle doit se elasser daus le voisinage du $F$. angustifolia C. B. Clarke (- IIenichlaena angustifolia Schrader). Elle se différencie à premicre vue par la longueur des épillets, qui atteignent $12 \mathrm{~mm}$. environ de long chez le $\mathrm{r}^{\prime}$. angustifolix et $35 \mathrm{~mm}$. ehe\% la plante nouvelle.

Nous examinerons après aroir donné les earactères du $F$. minutiflora C. B. Clarke, figuré dans la phanehe suivante, les différences des sousgemres et des espèces assez nombreuses distribuées dans le sud de l'Afrique. 




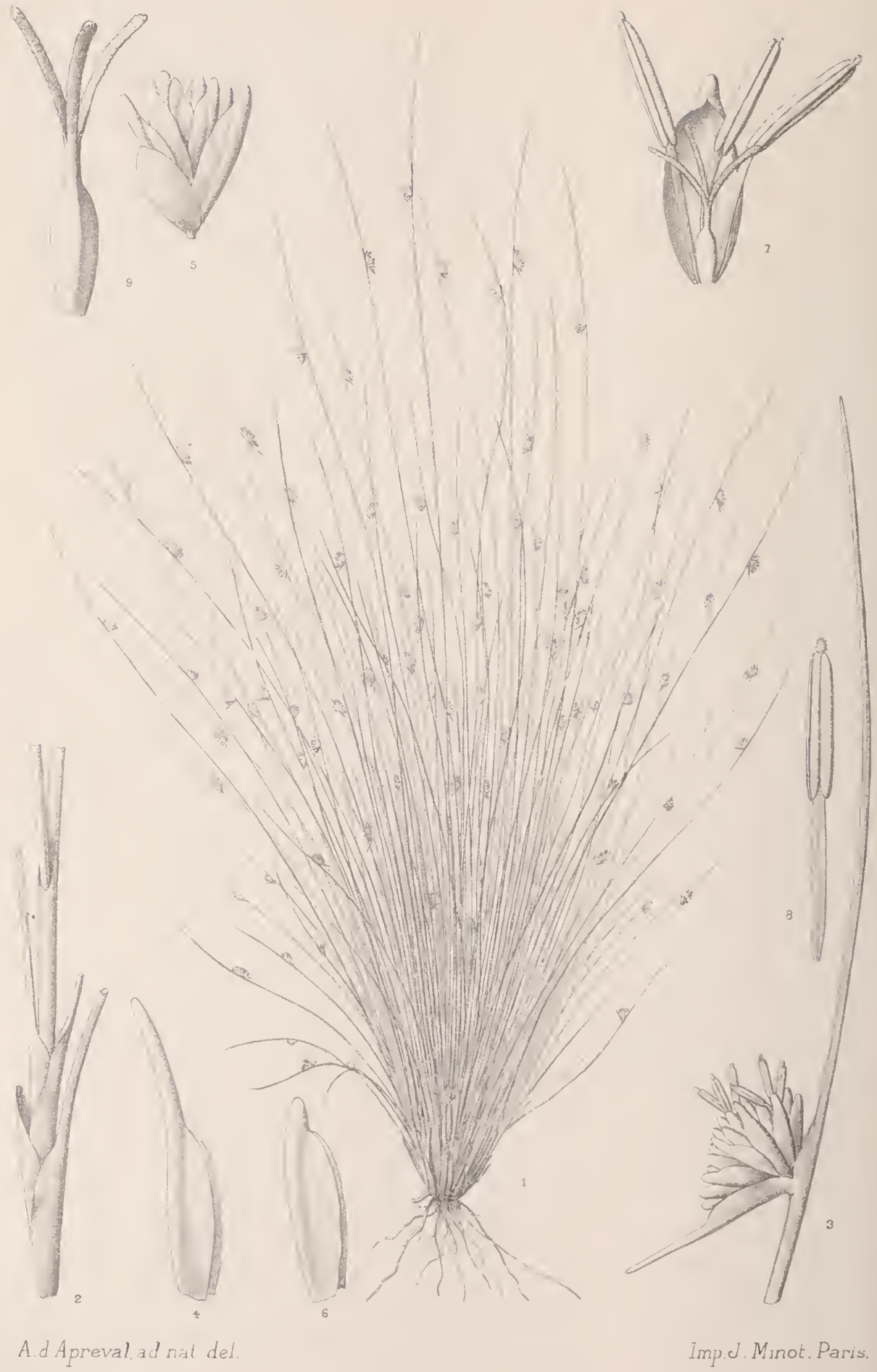




\section{Ficinia minutiflora C. B. Clarke}

in This. Dyer Fl. Cap. VII (1900) p. 759.

Plante glabre, à rhizome trés réduit, cespiteuse, formant des touffes de 5 à $20 \mathrm{~cm}$. de haut. Feuilles sétacées engainantes à la base, parfois aussi longues que les tiges. Capitule florifère latéral, de 2-3 mm. de diamètre, constitué par plusieurs épillets, entouré par deux bractées; l'une dans la direction de la tige, élargie à la base, longuement lancéolée au sommet, aiguë, atteignant plus de $2 \mathrm{~cm}$. de long; l'autre déjetée, environ deux fois aussi longue que le capitule. Expillets de $2 \mathrm{~mm}$. environ de long, pauciflores, d'un jaune pâle, blanchâtres. Glumes ovales, obtuses ou à carène obtuse terminée par un mucron peu proéminent. Étamines à filet environ aussi long que le style et les stigmates, à anthìre aussi longue que le filet, terminée par un apicule globulenx. Ovaire trigone, glabre, à style court, terminé par trois stigmates plus longs que le style.

\section{Explication des figures de la planche VIII.}

FIG. 1. - Plante entière (1/1).

FIG. 2. - Base d'une tige avec ses feuilles engainantes (6/1).

Fı. 3. - Capitule avec ses deux bractées $(6 / 1)$.

Fro. 4. - Bractée basilaire (20/1).

FıG. 5. - Épillet non mûr, isolè (15/1).

Fig. 6. - Glume légèrement et obtusement mucronée $(20 / 1)$.

FiG. 7. - Fleur avec sa glume (20/1).

Fia. 8. - Étamine vue de face (20/1).

Flg. 9. - Ovaire isolé (40/1). 
Hab. - Afrique australe : Rẻgion côtière austro-occidentale (division Caledon), Houw Hoek Mountains, 2,500 pieds (Galpin et Schlechter).

Observations. - M. C. B. Clarke a publié la deseription de eette espèce dans les “Addenda et eorrigenda " de la Flora of tropical $\Lambda$ frica. L'espèce est voisine du $F$. albicans Nees. Comme cette dernière espèce le $F$. minutiflora possède des glumes très obtuses ou à carène à peine et obtusément proéminente, des épillets blanehàtres très réduits dépassant rarement 2 à $3 \mathrm{~mm}$. de long; mais tandis que ehez le $F$. albicans les glumes seraient pointues, elles sont obtuses ehez le $F$. minutifora. Nous ne pouvons pousser davantage la différenciation de ces deux espèces, car tandis que la clef analytique proposée par M. C. B. Clarke porte "glumes pointel ", la description dit: "glumes ovate, the thick greenwhite keel subexeurrent as an obtuse mucro *, caractere aui concorde fortement avec ceux de la plante que nous figurous iei, comme le font voir du reste les figures $3,5,6$ et 7 de notre planche.

$$
*^{*} *
$$

Le genre Fieinia créc par Schrader et admis par M. C. B. Clarke, a été considéré comme sous-genre du grand genre Seirpus. Il diffère uniquement, comme le fait ressortir le spécialiste anglais, par le gynophore obpyramidal très réduit. Mais il est des plus difficiles de marquer une ligne de séparation nette entre certains Seirpus et quelques Fieinia; aussi des auteurs ont-ils considéré plusieurs Ficinia comme appartenant au genre Seirpus. I.es trois espèees de Fieinia que nous avons décrites et figurées, $F$. mueronata, distrns et minutiflora, appartiennent, la première et la dernière à un même sous-genre; la seconde, dont le port est tout différent, appartient à un groupement qui, comme nous l'arons déjà dit, a été eonsidéré eomme genre.

Le genre Fieinia, tel qu'il est eompris par C. B. Clarke, comprend environ 60 espèees dont la presque totalité se trouvent confinées en Afrique australe.

Le genre est divisé en cinq sous-genres, dont le premier, Sickmannia, se distingue très facilement grâce à son style très allongé, non divisé au sommet. Le $F$. ixioides Nees est seul à constituer un sous-gemre caractérisé par des épillets à fleur centrale fertile et à fleur latérale mâle. Dans les Eu-Fieinia, sous-genre auquel appartiennent les $F$. mueronata et minutiflora, les différences reposent sur la présenee de feuilles toutes basilaires et sur les glumes disposées en spirale; les branehes du style sont longues, comme d'ailleurs dans les autres groupements du genre que nous allons examiner. Dans ee sous-genre, qui eomprend le plus grand 
nombro d'espèces, M. Clarke-a créé deux subdivisions en se basant sur le nombre d'épillets contenus dans le capitule. Comme le montrent les figures de notre planche il n'y a chez le $F$. mucronata qu'un seul épillet, c'est-ì-dire que cette espèce vient se classer dans le groupe des I'. scariosa Nees, Zeyhtri Boeck., pusilla C. B. Clarke, micrantha C. B. Clarke, et, nous basant sur la clef analytique proposée par M. Clarke, nous pourrons la modifier, par suite de l'introduction de l'espèce nouvelle, comme suit:

Tiges à un épillet.

Tiges fortes; épillets grands parfois de $24 \mathrm{~mm}$. F. scariosa.

T'iges gr'êles.

Eipillets de $6 \mathrm{~mm}$. environ de long . . . F. Zeyheri.

Épillets de $4 \mathrm{~mm}$. environ de long; gaine supérieure non feuillée.

Glumes obtuses . . . . . . F.pusilla.

Glumes obtuses à mucron rigide . . . $F$. mucronata.

Épillets de $4 \mathrm{~mm}$. environ de long; gaine

supérieure feuillée . . . . F.micrantha.

Le $h$. minutiflora appartient lui au second groupement car, comme le montrent nos dessins, le capitule r'enfer'me plusieur's épillets, et dans cette subdivision, il se range avec la plupart des espèces dans le groupe à style trifide. Par la présence de feuilles, par le nombre d'épillets de 1 à 6 , disposés en éventail, le $F$. minutiflora appartient au groupe du $F$. albicans, caractérisé par des glumes obtuses, des épillets petits, dépassant rarement $4 \mathrm{~mm}$. de long. Sans entrer dans la différenciation des nombreuses espèces de ce groupe, nous classerons cornme suit cette plante parmi ses congénères :

Épillets petits d'environ $4 \mathrm{~mm}$. de long.

Épillets d'un brun foncé.

Gaine foliaire marginée de blanc . . F. filiformis.

Gaine foliaire d'un brun pale . . . $F$. Beryiana.

Épillets d'un ver't blanchâtre.

Glumes aiguës . . . . . . . F. albicans.

Glumes obtuses ou à pointe obtuse. . . $r$. minuliflora.

Nous rappelons ici, au sujet de cette différenciation, la remarque que nous avons faite plus lıaut au sujet du désaccord qui semble régner entre la clef anilytique et la description de la F'lora Capensis.

Quant au $F$. distuns, il appartient, comme nous l'arons dit, au sousgenre Ilemichlona, caractérisé prar ses tiges plus ou moins ramitiées, ses épillets allongés, ses giumes distiques. 
La clef analytique proposée par M. Clarke pourrait être modifiée comme suit, en y intercalant l'espèce nouvelle:

Tiges simples formant des touffes, nœuds foliaires près de la base; épillets digités. Épillets de $12 \mathrm{~mm}$. de long . . . . F. angustifolia. Épillets atteignant $35 \mathrm{~mm}$. de long . . . $F$. distans. Tiges ramifiées, nœuds foliaires éloignés de la base (Cf. C. B. Clarke in This.-Dyer Fl. Cap. VII p. 237). . . . . . . . $F$. longifolia. F. capillifolia. 



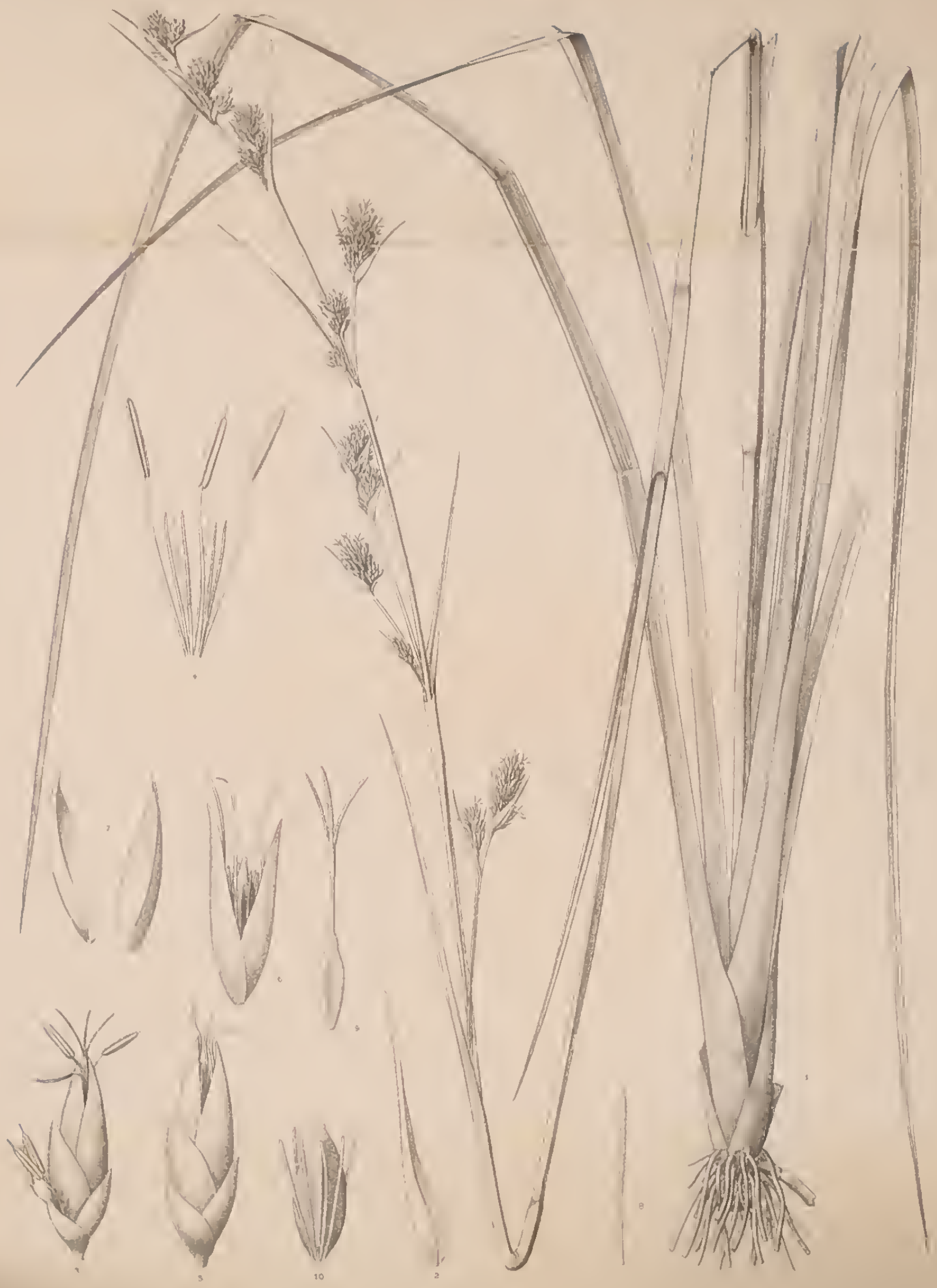




\section{Carpha Schlechteri C. B. Clarke nov. sp.}

Carpha Schlechteri; C. glomerate Nees affinis; differt : foliis angustioribus (vix $5 \mathrm{~mm}$. latis); inflorescentia graciliore, spiculis minoribus; nuce grosse conspicue hexagono-reticulata levi (neque, ut in C. glomerata, minute obscure reticulata).

Plante assez robuste, glabre, sauf sur le dos des glumes florales ciliées. Rhizome inconnu. Tige atteignant $80 \mathrm{~cm}$. de haut, trigone, munie de nouds peu proéminents et le long de la tige de liractées espacées sous la portion florifère. Feuilles engainantes à la base, de $40-50 \mathrm{~cm}$. de long et de $5 \mathrm{~mm}$. environ de large, assez lisses, à striation longitudinale faible. Inflorescence assez grêle, de $25 \mathrm{~cm}$. environ de long, formant une panicule peu élargie, à ramifications espacées. Bractées primaires analogues aux feuilles, assez longuement engainantes à la base, dépassant en général les ramifications. Bractées secondaires lancéolées, aiguës, carénées sur le dos, ciliées. Capitules ovoïdes, d'un jaune brunâtre, de 1 à $1,5 \mathrm{~cm}$. de long; épillets formés souvent de plusieurs fleurs dont les inférieures sont mâles ou avortées, la supérieure fertile. Glumes de la fleur fertile naviculi-

\section{Explication des figures de la planche IX.}

Fig. 1. - Plante entière (1/1).

Fıc. 2. - Bractée de la base de l'épi $(8 / 1)$.

FiG. 3. - Épillet, dont une des glumes inférieures contient une fleur mâle (10/1).

Fıa, 4. - Filaments hypogynes et étamiues d'une fleur mále (20/1).

Fo. 5. - Ĺpillet dont toutes les glumes inférieures sont privées de fleurs (10/1).

Fia. 6. - Fleurs fertiles dont les anthères sont tombées (10/1). 
formes, glabres, aiguës, mucronées, entourant 6 filaments liypogynes dont 3 représentent les pétales, 3 les sépales, linéaires, rigides, scabres dans leur partie supérieure, subégaux, plus courts que les filets staninaux. Etamines au nombre de 3 , à anthères terminées par un prolongement aigu. Ovaire trigone, brunâtre, à style muni à la base à l'état jeune d'un renflement pyramidal, velu ; à maturité la base du style devient confluente avec le mucule, celui-ci est scabre sur le bord et muni sur les parois d'une réticulation hexagone assez nette. Style a trois stigmates aussi longs que le style, se désarticulant à maturité juste au-dessus de l'ovaire.

Hab. - Afrique australe. - Région austro-occidentale : Koude Bokkeveld, Gydow à 4,500 pieds (R. Schlechter, 17 janvier 1897).

Olserrations. - Comme le fait remarquer M. C. B. Clarke, dans la courte diagnose qui figure en tete de cette notice, ce Carpha est voisin du C. glomerula Nees, dont il liffere surtout par le port plus grèle et par un nomdre développement des diverses parties de la plante; il existe égralement dans le nucule un caractère assez tranché, les faces du nucule sont dans l'espece nouvelle marquees d'une reeticulation hexagonale assez forte, bien visible à la loupe, chez le c'. glomerata la réticulation existe légìrenent, mais beaucoup moins visible.

Nous reviendrons sur les caractères de cette plante et sur les espèces voisines peu nombreuses en Afrique australe, en examinantle C.bracteosa c. B. Clarke, dont nous figurons ci-après un spécimen provenant du mème collecteur.

Fia. 7. - Glumes de la fleur fertile isolées (9/1).

Fia. 8. - Soie hypogyne isolée (12/1).

Fía. 9. - Ovaire jeune avec style renflé à la base (13/1).

F'IG. 10. - Nucule adulte privé du style, entouré des soie liypogynes (10/1). 



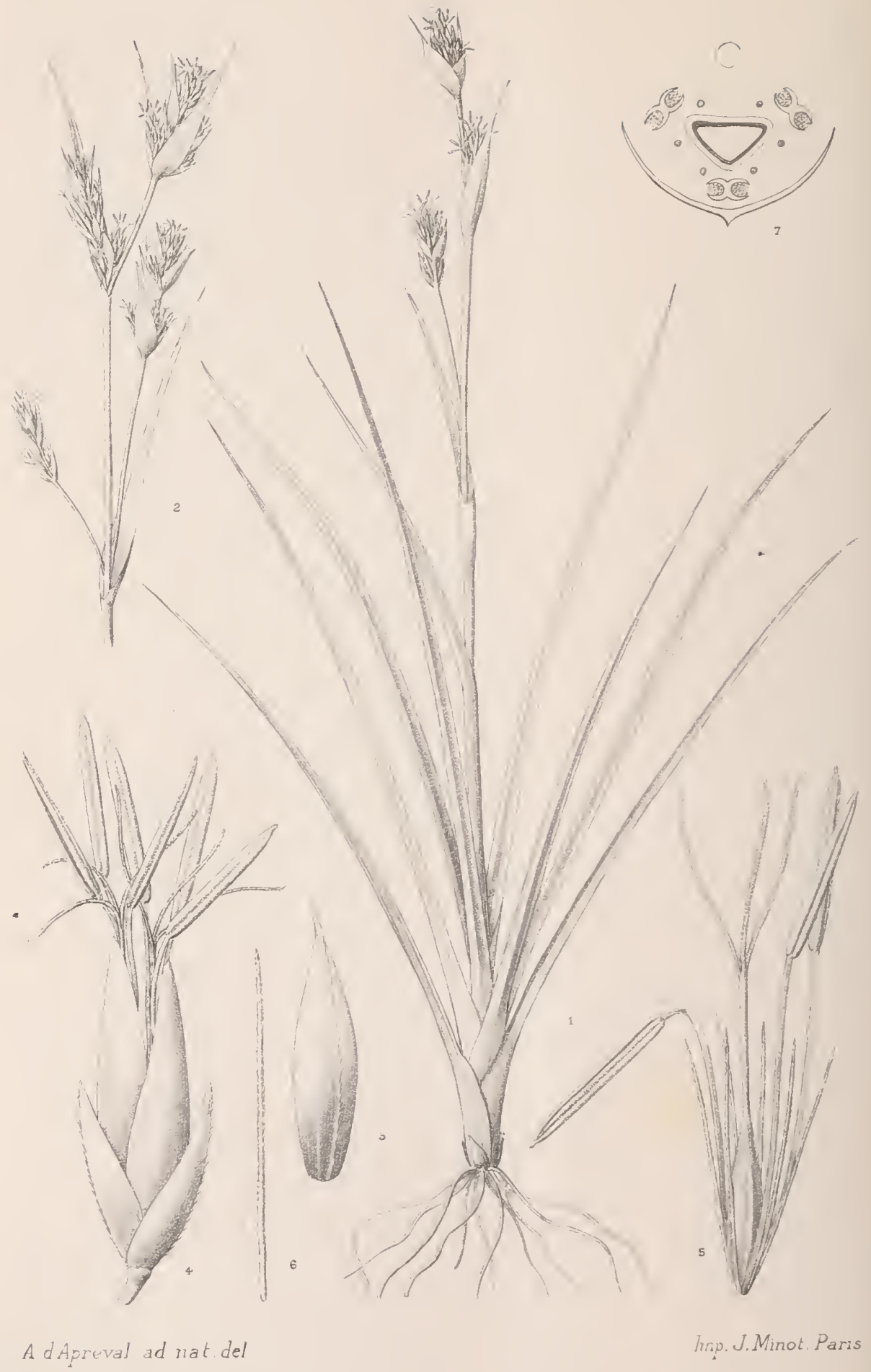

CARPHA BRACTEOSA C.B.Clarke 


\section{Carpha bracteosa C. B. Clurkie}

in Durand et Schinz Conspectus Fl. Afric. V (1895) 1. 656, n. nudum et in This.-Dyer Fl. Cap. VII (1898).

Plante assez grêle, glabre dans toutes ses parties sauf sur le dos des glumes légèrement liispides. Feuilles au nombre de 4 à 12 , de 10 à $30 \mathrm{~cm}$. de long et $1,5-3 \mathrm{~mm}$. de large. Inflorescence de $15-35 \mathrm{~cm}$. de long, constituée par une série de capitules plus ou moins pédicellés formant une panicule plus ou moins ramifiée. Bractées de la base de l'inflorescence plus ou moins engainantes; bractées de la base des capitules ovales-aiguës, à bords scarieux, aiguës au sommet, carénées sur le dos et ciliées sur la carène; épillets au nombre de 3 à 8 , de $8.10 \mathrm{~mm}$. de long, entourés par la bractée externe qui est aussi longue, plus courte, ou plus longue que l'épillet. Glumes de même forme que les bractées et ciliées sur le dos, entourant deux fleurs. Glumelles fructifères naviculiformes, glabres même sur le dos, dépassant l'ovaire. Filaments hypogynes au

\section{Explication des figures de la planche X.}

Fig. 1. - Petite plante entière (1/1).

FIg. 2. - Extrémité d'un rameau florifère, pris sur un pied un peu plus développé (1/1).

Fig. 3. - Glume de l'épillet vue de dos (5/1).

FıG. 4. - Un épillet muni de ses glumes externes renfermant deux fleurs (10/1). 
nombre de 6 , représentant les pétales et les sépales, linéaires, rigides, scahres, subégaux, aigus. Étamines au nombre de trois, i filets plus longs que les filaments lıypogynes, à anthère allongée, un peu plus courte que le filet. Ovaire de $1.5 \mathrm{~mm}$. environ de long, ellip. tique, trigone, glabre a la base, courtement pubéruleux au sommet. Style cylindrique, environ aussi long que l'ovaire, glabre, terniné par 3 stigmates aussi longs que le style, étalés.

Hab. - Afrique australe : Région austro-occidentale - Worcester division : Vallée de la Breede-River à Bains-Kloof, 800 pieds (H. Bolus). Mitch ls-Pass, 1.000 pieds (R. Schlechter, n. 8970, 11 septembre 1896). - Région austro-orientale (limite extrêmel. Somerset division : Bosch-Berg, 4.500 pieds (MacOwan).

Obscrvations. - I.e genre Carpha crié prar R. Brown et modifié dans sa compréhension par M. C B. Clarke ne renferme que peu d'espèces; on n'en rompte gnère qu une dizaine confinées lans l'hémisphère sud, e'est-à-dire dans le sud de l'Afrique, les iles Wascareigurs. l'Australie et l'Amérique tempérée australe. Ce gemre se caractérise. peut-on dire, par ses pétales et sépales remplacés par des soies liyjogrynes simples, par ses tiges florifères munies de nneuds et de feuilles éparses.

La distinction des espèces n'est guère facile dans ce genre. comme d'ailleurs dans les autres genres de Cypéracées. M. C. B. Clarkie dans la revue monographique des Cypéracèes qu'il a prubliée dans la Flora capensis de Sir William 'Thiselton-Dyer, s'est basé, pour différencier les 3 espèces admises à ce moment dans cette flore, sur le nombre d'épillets et le développement de la panicule florifère et sur la largeur des feuilles. Nous basant sur les mêmes caractères, nous pourons dès lor's ¿tablir comme suit la clef analytique des espèces africaines de ce genre après intercalation de la plante nouvelle, décrite et figurée précédemment :

Fra. 5. - Fleur privèe de sa glumelle montrant la situation relative des filaments, des étamines et de l'ovaire (19/1).

Fıg. 6. - Filament hypogyne isolé (12/1).

Fia. 7. - Schéma d'une fleur. 
Capitules d'épillets plus ou moins nombreux, formant une panicule assez développée; feuilles de 5 à $12 \mathrm{~mm}$, de large.

Feuilles de 4-12 mm. de lirrge; uucule lisse obscurément réticulé . . . C. glomerata.

Feuilles de 4-5 min. de large; nucule à l’éticulation hexagonale bien nette. . Capitules d'épillets peu nombreux, formant une C. Schlechteri. courte panicule; feuilles de moins de $6 \mathrm{~mm}$. de large.

Bractées des capitules peu risibles, lancéolées, rétrécies à la base . . . . C. capilellata.

Brictées des capitules ovales ou subcorlées à la base . . . . . C. bracteosa.

Les derniers caractères sont ceux admis par M. Clarke; la planche ci-contre fait suffisamment ressortir les caractères des bractées, elle fait voir également que la différenciation basée sur la panicule est difficile à saisir, car, comme le montrent les figures 1 et 2 , sur des pieds différents appartenant à la méme plante on peut trouver des variantes très nettes dans le développenent de la panicule, celle-ci est beaucoup plus ample dans la figure 2 que dans la figure 1, sans encore atteindre, est vrai, le développement de la panicule de l'espèce figurée précédemment.

Aucune espèce africaine de ce genre n'avait été figurée jusqu'à ce jour; c'est la raison pour laquelle nous avons été heureux de faire reproduire ici ces dessins, ils mettent en vedette des caractères difficiles à apprécier. 



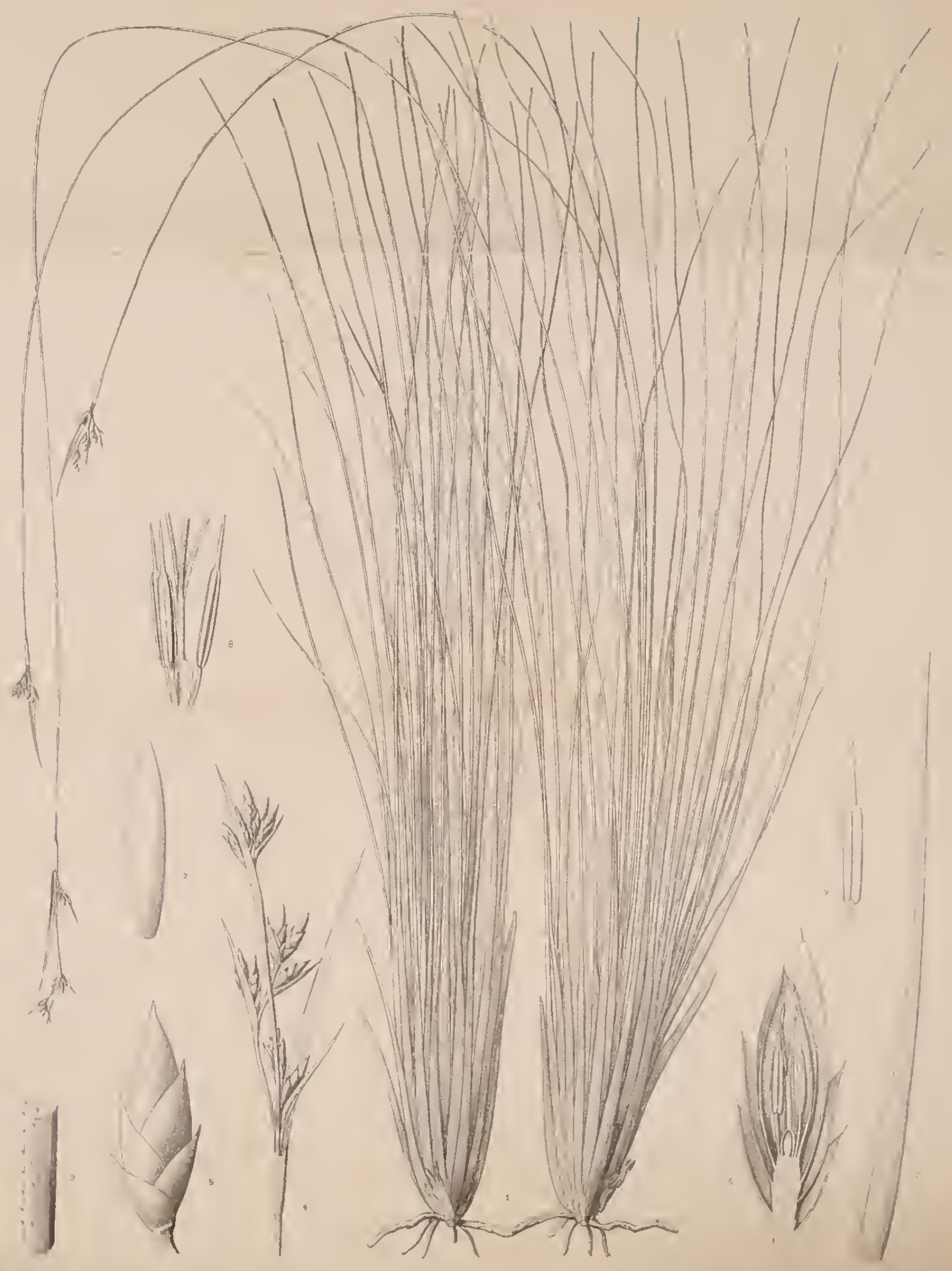


\section{Tetraria ferruginea C. B. Clarlie.}

in This.-Dyer Fl. Cap. V (1900) p. 759.

Plante glabre à rhizome horizontal, de $3-4 \mathrm{~mm}$. de diamètre. Tiges rapprochées, d'environ deux pieds de haut, grêles, sans nœuds sauf près de la base, formant des touffes espacées. Gaines inférieures ferrugineuses-brunâtres, plus ou moins viscides, feuilles de $30 \mathrm{~cm}$. de long, filiformes, courtement aiguillonnées sur les hords, canaliculées. Panicules de 2 à $5 \mathrm{~cm}$. de long, formées de 12 à 44 épillets, bractéolés à la base; bractée inférieure dépassant l'inflorescence, à gaine assez développée, ferrugineusebrunâtre. Épillets pris isolément à 5-8 glumes dont toutes les inférieures sont stériles, la supérieure seule, bi-sexuelle, fertile; glumes inférieures glabres, légèrement ciliées sur les bords; glumes supérieures longuement ciliées sur les bords; toutes non aristées, légèrement mucronées. Étamines au nombre de 3 , à filet un peu plus long que l'ovaire,

\section{Explication des figures de la planche XI.}

F10. 1. - Deux touffes fleuries sur un fragment de rhizome $(1 / 1)$.

Fig. 2. - Base d'une feuille (3/1).

Fio. 3. - Fragment de la feuille vu de profil (12/1).

FiG. 4. - Extrémité du péloncule florifère avec bractèe engainante (3/1).

FiG 5. - Épillet isolè (12/1).

Firi. 6. - Coupe longitudinale de l'épillel (15/1).

Fic. 7. - Une des glumes de la feur fertile, vue de profil (15/1).

FIG. \&. - Fleur bisesuelle $(201)$.

Fio. 9. - Anthère mucronée (23/1). 
anthère un peu plus longue que le filet, terminée par une ariste aiguë. Ovaire trigone à style environ aussi long que les trois stigmates.

Hab. - Afrique australe : Région austro-occidentale, division Ceres-Bokkeveld, Elandsfontein, 5,000 pieds, 18 janvier 1897 (R, Schlechter).

Ors. - Le genre Tetraria créé prar le botaniste français Palisot de Beauvois comprend 31 espèces endémiques dans le sud de l'Afrique et une espèce qui se rencontre dans la zone florale du Cap et dans l'Usambara. Ces 31 espèces se rangent dans six sections que M. Clarke décrit comme suit :

I. - Aulacorhynchus C. B. Clarke (pris comme genre par Nees).-Épillets tous unisexués, monoüuues, femelles, uniflores; glumes non aristées; étamines 3 ; style ì 3 branches; tiges et feuilles grèles (unique espèce $T$. crinifolia C. B. Clarke).

II. - Hemischoenus C. B. Clarke. - Fleur fertile bisexuelle; fleur inférieure donnant un akène; épillets généralement uniflores; glumes non aristíes; étamines 3 ; style trifide; tiges et feuilles grêles, ces dernières toutes basilail'es (unique espèce $\mathbf{T}$. cuspidata C. B. Clarke).

III. - Elynanthus C. B. Clarke (genre pour plusieurs auteurs).

- Fleur fertile bisexuelle; fleur inférierre ne produisant pas d'akène; glumes non aristées, à peine mucronées (sauf dans le $T$. aristata); étamines 3 ou 4, styles à 3 ou 4 branches; plante grèle ou de force moyenne à feuilles étroites.

IV. - Lepisia C. B. Clarke (genre de Presl). - Fleur fertile bisexuelle; l'inférieure ne formant pas d'akène; glumes infélieures aristées; étamines au nombre de 3 ou 4 ; style à 3 ou 4 branches; plantes vigoureuses à feuilles planes; tiges à nœuds portant des feuilles ou des bractées dispersées le long de la tige.

V. - Eu-Tetraria C. B. Clarke. - Fleur fertile bisexuelle; fleur inférieure mâle avec un pistil rudimentaire, ne formant pas de graine; glumes distiques non aristées ; étamines 6 ou 8 ; style à 3 à 4 branches: plantes vigoureuses ou de force moyenne.

VI. - Buekia C. B. Clarke. - Fleur fertile bisexuelle; fleur inférieure formant un akène; glumes à peine distiques, très légèrement mucronées; étamines 3 ou 6 à anthères munies d'une crête; style à 6 ou $\delta$ branches; tige robuste, à nœuds entre les feuilles basilaires et la panicule. 
Si l'on comprare ces données avec celles de la plante ci-jointe, on verra que la plante figurée appartient à la section III qui r'enferme d'ailleurs le plus grand nombre d'espèces. M. C. B. Clarke a rangé les espèces de ce sous-genre en deux groupes :

Tiges sans nœuds entre la bractée inférieure de l'inflorescence et les feuilles presque basilaires.

Tiges avec un ou plusieur's nouds entre la bractée inférieure de l'inflorescence et les feuilles basilaires.

Ce sera naturellement dans la première de ces divisions que l'on rangera l'espèce nouvelle que $M$. Clarke n'a pu introduire dans la clef analytique des espèces du genre. M. C. B. Clarke considère le T. ferruginea comme voisin des T. pleosticha C. B. Clarke et Wallichiana C. B. Clarke, et se base sur les caractères suivants pour séparer 5 espèces des deux l'appelées ci-dessus :

T.eaf sheats not manifestly fimbriate.

Leaf sheats fimbriate or concellate.

Ces derniers caractères apluartiennent aux T.pleosticha et Wallichiana, mais ne corresprondent guère avec ceux ligurés sur notre planche; en effet si parfois dans les échantillons on peut obserrer' des fibrilles sur le bord des gaines, ces fibrilles proviennent de la décomposition des feuilles déjà très adultes; dans les feuilles jeunes les gaines sont à bol'ds entier's.

Le $T$. Wallichiana possède une panicule compacte, le $T$. pleosticha une panicule làche, le $T$. ferruginea serait intermédiaire; en l'absence des matériaux authentiques de ees deux espèces, il serait difficile de pousser plus loin la différenciation des trois plantes. 






\title{
PLANT $\nRightarrow$ NOV E
} VEL MINUS COGNITÆ

FA IIERBARIO HORTI THENENSIS

\section{PLANTES NOUVELLES OU PEU CONNUES}

(ONTENUES DANS LHERBLER

DE

L'HORTUS THENENSIS

\section{AVEC LES DESCRIPTIONS OU ANNOTATIONS}

Dl:

M. Ém. DE WILDEMAN

DUCTEUR EN SCIENCES

CUNSERVATEUR AU JARDIY BUTAYIQUE DE L'ÉTAT

\section{Tromideme livraniman.}

(DECEMBRE 1904)

\author{
VEUVE MONNOM

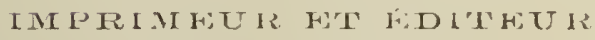 \\ 32, RUE DE L'INDUSTRL, 32 \\ BRUXELLES
}

Se vend exclusivement à l'Administration des biens et affaires de M. van den Bossche, 5, Grande Montagne, à Tirlemont. 



\section{TROISIEME LIVRAISON}

Comme la première, la troisième livraison de cette publication est exclusivement consacrée à cette partie de l'Herbier de l'Ilortus Thenensis qui contient les plantes lécoltées au Mozambique par M. Éd. Luja.

Nous avons cru qu’il y avait intérêt à gronper dans me même livraison toutes celles de ees plantes qui appartiemnent à la grande famille des Orchidacées et qui nous ont offert des éléments de détermination snlfisants pour en établir une diagnnose satisfaisante. Nous avons pu ainsi faire dessiner dix planches, domnant des analyses détaillées de onze espèces différentes, dont sept sont pon1 nons des espèces nonvelles, non encore décrites; les quatre autres, déjic connues par les descriptions des auteurs, n’avaient pas été figurées jusqu’à ce jour.

M. le 1) De Wildemaun a cher'ché à attirer l'attention des savants sur' les unes et les autres, en s'attachant surtout, an cours de ses observations, à établir les allinités aree les esperes voisines.

I. B. 


PSOVAE HFRI HKRT THENENSIS

PL.XII
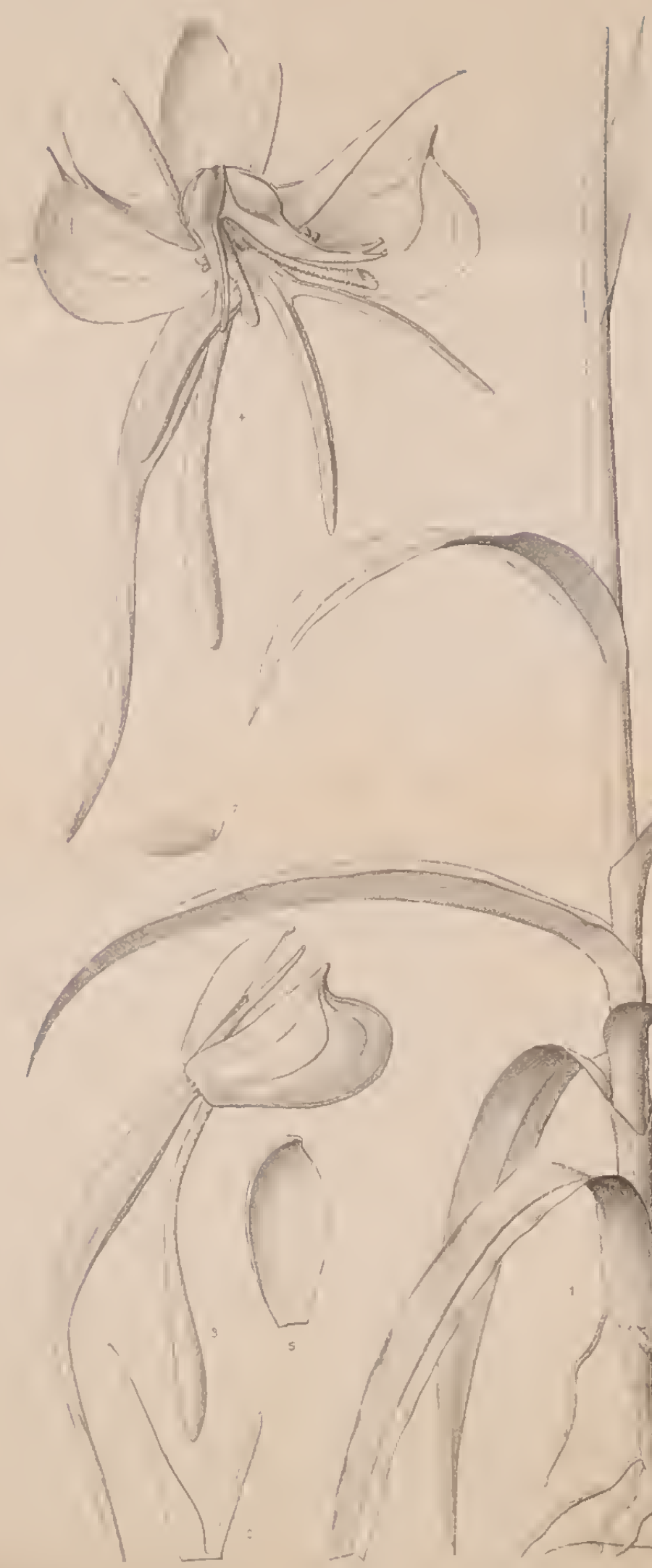

i tripievar sa rat des

Irt $J$ Minar Parzs

HABENARIA PEDICEILIAARTS ReIrh \& 


\section{Habenaria pedicellaris Reichb. f.}

()tia Bot. Hamb. II (1878) p. 100; Kränzl. in Engl. Jahrb. XVI p. 78, et in Engl. Pflanzenw. Ost-Ifr. C p. 152; Rolfe in This.-Dyer Fl. trop. Afr. VII p. 214 ; Kränzl. Orchid. gen, et spec. I p. 221.

Plante herbacée, atteignant $50 \mathrm{~cm}$. de long, à tubercule ovoülle, à racines velues nombrenses. Feuilles caulinaires au nombre de 6 ì 8 , linéaires-lancéolées, aiguës, engainantes à la base, de 7 à 15 con. de long et 5 à $12 \mathrm{~mm}$. de large. Racèmes atteignant plus de $20 \mathrm{~cm}$. de long, à bractées lancéolées, acuminées, aiguës, atteignant ì la base du racème $25 \mathrm{~mm}$. de long. Fleurs à pédicelles plus longs que les bractées, à sépale dorsal elliptique-oblong, mucronéobtus, de 5 mm. environ de long; sépales latéraux obliquement obovales-oblongss, apiculés à l'angle supérieur, de 6-7 $\mathrm{mm}$. de long et environ aussi larges. Pétales bipartites ì lobe postérieur aussi long que le sépale dorsal, linéaire-filiforme, courtement cilié ; lobe antérieur de 8 à $9 \mathrm{~mm}$. de long, légèrenent élargi à li base. Labelle protondément trijartite, à lobes linéaires, filiformes, de $8 \mathrm{~mm}$. environ de long, subégaux ou le médian un peu plus long que les latéraux. Éperon de 15 à $20 \mathrm{~mm}$. de long, filiforme,

Explication des figures de la planche XII.

Fo. 1. - Base de la tige florifère (1/1).

Fra. 2. - Extrémité de la tige arec inflorescence (1/1).

Fig. 3. - Fleur peu avant épanouissement.

Fio. 4. - Fleur épanouie (4/1).

Fir. 5. - Sépale dorsal vu par sa face interne (4/1). 
légèrement renflé en massue à son extrémité. Processus stigmatiques claviformes, obliquement tronqués au sommet, de $4 \mathrm{~mm}$. enriron de long. Canaux anthérifères grêles, aussi longrs que les stigmates. Oraire à trois côtes ailées et trois côtes simples, celles-ci en rapport avec les placentas.

Hab. - Région nilienne : Abyssinie (environs dủ lac Tano).-Région du Mozambique : Kilimanjaro (Marangu) et Morrumbala (Zambése) 1,000 mètres d'altitude, avril 1901 (Éd. Luja, n. 385 .

Observations. - Le genre Habenaria est un des plus riches en espèces; sa dispersion est d'ailleur's très vaste puisqu'il est représenté dans la flore de l’Amérique tropicale et de l'Asie tropicale, et que dans le rontinent africain, il se rencontre depuis l'A byssinie jusque dans la rolonie du Cap. Sur les trois à quatre cents espèces qui le constituent, plus de cent appartiement à la flor'e de l'Afrique tropicale et ce nombre augmente constamment avec les explorations botaniques faites dans le continent noir.

Il est liun des genres les plus embrouillés de la grande famille des Orchidacées; malgré les nombreux travaux qui ont été publiés, les auteurs sont loin d'itre d'accord sur des points importants, et pour ne citer qu'un exemple, M. Liränzlin, dont la compétence en matière d'IIabenaria doit ìtre r'econnue, considère dans son Genera, l'H. culicifera Rendle comme synonyme de l'H. pedicellaris, alors que M. Rolfe, le savant monographe anglais, classe ces deux plantes très loin l'une de l'autre.

Nous n'avons pas à passer en rerue ici les différentes sections du genre qui ont été proposées par les divers auteur's, cela nous mènerait trop loin et n'aurait d'ailleur's pas grand intérèt, car nous ne pourrions étayer nos remarques sur des matériaux suffisants. Il nous suffira d’attirer l'attention sur les principaux caractères distinctifs de notre plante que nous avons fait figurer parce qu'elle ne l'avait pas été jusqu'ici et qu'elle fait l'objet d'une divergence d'opinion entre MM. Rolfe et Kränzlin; en outre, nous avions certains détails de la fleur à faire ressortir, car les mensurations que nous arons observées ne concordent pas avec celles publiées antérieurement.

Fıs. 6. - Petale bipartite (4/1).

Fic. 7. - Anthère fortement grossie.

Fı. 8. - Coupe lransversale de l'ovaire (10/1). 
Sur los cent sept espèces signalies prar Ml. Liolfe dans la fFloria of tropical Africa, vol. VII, quarante-deux possèdent des lleur's à pétales cntiers, tontes les autres ont des pétales bilobés ou bipartites comme ceux de la plante que nous figurons (ef. fig. 6). I.es feuilles caulinaires lancéolées, l'éperon relativement court, ne dépassant pas $2.1 \mathrm{~mm}$. de long, les processus stigmatiques d'environ $4 \mathrm{~mm}$. au moins de long, les lobes du labelle de 8 a $10 \mathrm{~mm}$. de long, classent la plante que nous signalons ici dans un groupe constitué, d'après les données de M. Rolfe, par les $H$. Hochstelleriana Kränzl. (A byssinie), H. pedicellaris Reichb. $f$. et $H$. ranicolorala Reichb. f. (Kilimanjaro) La différenciation de ces trois espèces est relativement facile; en effet, chez la première d'entre elles les processus stigmatiques sont grèles et capités, tandis qu'ils sont claviformes et obliquement tronqués au sommet chez les deux dernières, comme le montre trés neltement la figure 4 de notre planche NII. La différence de ces espèces réside en particulier dans la longueur de ces processus; ils atteignent 4 mun. environ chez l'11. pedicellaris, et chez l'H. ranicolorata enriron $6 \mathrm{~mm}$. 




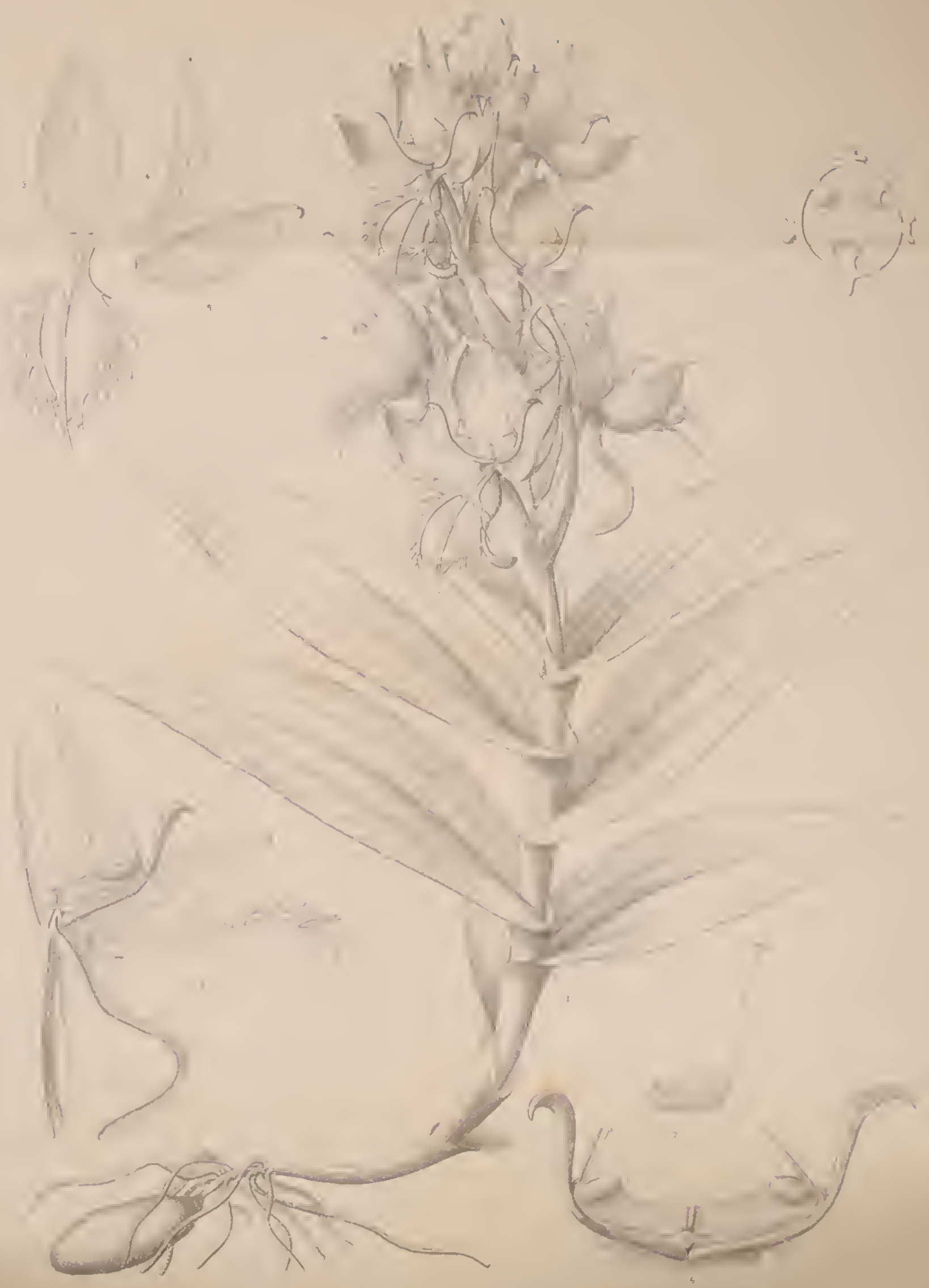




\section{Habenaria splendens Rendle}

in lourn. Ism. Soc XXX (1895) p. 395); Rolfe in This.-Dyer F'. trop. Alr. V'Il p. 221.

Plante ter'estre, de 30 it $40 \mathrm{~cm}$. de haut, à tubercule ovoïle, a ralcomes groiles. 'Triges dressées a fenilles remplaceées it la base par des bractées enganantes à limbe rédnit. Frenilles ovales on ovales-lancéolées, subaiguës, plus ou moins jlissées longitudinalement, diminnant de grandem du milieu de la tige ver's le sommet où elles se changent en bractées, de 5 i 12 cur. de long et de 25 à $40 \mathrm{~mm}$. de large. Bractées ovales, aigruës, atteignant 4 cm. de long. Fleurs blanches disposées en épi terminal, a pédicelle de 25 a $35 \mathrm{~mm}$. de long. Sépale dorsal ovale, subaigu, de $25111 \mathrm{~m}$. environ de long, à nervation bien marquée, plus ou moins caréné; sépales latéraux subobliques, ovales-oblong's, apiculés, à pointe recounbée, nerviés comme le sépale terminal, un pen plus longrs que lui. Pétales obliques, lancéolés-oblonges, subarrondis au sommet, de 26 11nn. environ de long, nerviés. Labelle étroitement rétréci à la base en 11 onglet, de 8 i $10 \mathrm{~mm}$. de long, a limbe profondément trilobé, de $3 \mathrm{~cm}$. environ de

Explication des figures de la planche XIII.

Fia. 1. - Plante fleurie, grandeur naturelle.

Fia. 2. - Labelle vu de face $(1,5 / 1)$.

Fig. 3. - Sépale dorsal, face interne $(1,5 / 1)$.

Fra. 4. - Pérale (1,5/1).

Fra. 5. - Sepale latéral $(1,5 / 1)$. 
long, à segrment médian linéaire, entier, un peu plus lonor que les lobes latéraux; ceux-ci légrèrement arqués, fimbriés sur les bords. Éperon légèrement recourbé, subcylindrique, légèrement renflé à son extrémité. Processus stigmatiques de $18 \mathrm{~mm}$. environ de long, divergents. Caudicules des antlières grèles, libres, de $5 \mathrm{~mm}$. environ de longr. Rostellum ì lube central nul. Ovaire à 6 côtes proéminentes, 6 còtes simples alternant avec des côtes aplaties-ailées, les placentas longitudinaux correspondant aux cotes simples.

Hab, - District du Mozambique : Mochi et Morang (Kilimanjaro): Marangu.

A ces localitès il faut ajouter pour le même district : Morrumbala (Zambèse). 1,000 m. d'altitude, 20 avril 1901 (Éd Luja, n. 386).

Observalions. - L'espèce que nous venons de faire figurer et dont un bel exemplarre a été recueilli par M. Luja, parait assez rare; on ne la connaissait jusqu'à présent que dans les trois localités : Kilimanjaro (Johnston), au delà de Mosang (Taylor'), et dans le Marangu (Volkens), les premier's échantillons n'avaient pu ètre déterminés avec certitude et M. Oliver dans les 'Transactions de la société linnéenne de Londres, les avait lapportés avec donte à l'II. macrantha Reichb. f.

Sans entrer dans le détail de la différenciation des tribus, nous tenons cependant à donner les affinites de la plante que nous figurons et à ce propos, un coup d'œil jeté sur la figure 1 et la figure 4 fait voir immédiatement que l $H$. splendens appartient à un groupe tout différent de l'H. pedicellaris, puisque les pétales sont entiers. Dans ce groupe peu d'espèces ont le labelle entier, le plus grand nombre ont le labello trilobé ou tripartite, comme l'a très nettement d'ailleur's l'H. splendens (cf. fig. 2). Par les lobes latéraux du labelle pectinés ou timbriés, l'éperon de plus de $12 \mathrm{~mm}$. de long, les feuilles ovales-lancéolées ou oblongues, le sépale dor'sal de plus de $10 \mathrm{~mm}$. de long: notie plante appartient à un groupe de trois espèces: $H$. macrantha Hochst. (Abyssinie), H. splendens Rendle et $H$. praestans Rendle (Ruwenzori, environs de Blantyre).

liki. 6. - Organes sexuels vus de face (3/1).

Fic. 7. - Masse pollinique avec son caudicule $(9 / 1)$.

FIG. 8. - Coupe longitudinale de la fleur (2/1).

Fı. 9. - Coupe transversale de l'ovaire $(10,1)$. 
Cette dernière espèce est facilement reconnaissable par son éperou relativement court, mesurant $20 \mathrm{~mm}$. environ, tandis que che\% les deux autres espèces il mesure de 30 à $36 \mathrm{~mm}$. Quant aux $H$. macrantha et splendens, la longueur de l'onglet du labelle permet la différenciation; d'après M. Rendle les mensurations suivantes sont particulières ar $\mathrm{x}$ deux espèces:

Onglet du labelle de $5 \mathrm{~mm}$. de long . . . H. macrantha. Onglet du labelle de $10 \mathrm{~mm}$, de long. . . . H. splendens.

Il y a lieu de faire r'essortir, comme le montre déjà notre planche, que cette mensuration, varie légèrement; cet onglet ne mesure fréquemment que $8 \mathrm{~mm}$. environ, de sorte qu'il serait plus exact de dire : Onglet du labelle de 8 à $10 \mathrm{~mm}$. de long; en tous cas, cet onglet est toujours plus déreloppé chez l'H. splenderis qu'il ne l'est chez l'H. macrantha. L' $H$. splendens possèderait, d'après $M$. Rolfe, des fleurs plus grandes que celles de l'H. macrantha et par suite de la soudure des anthères avec les branches stignatiques sur une grande longueur, la portion libre des processus stigmatiques parait plus réduite. 

I' VINAR HRKH HORT IHINENSIS

P..X川

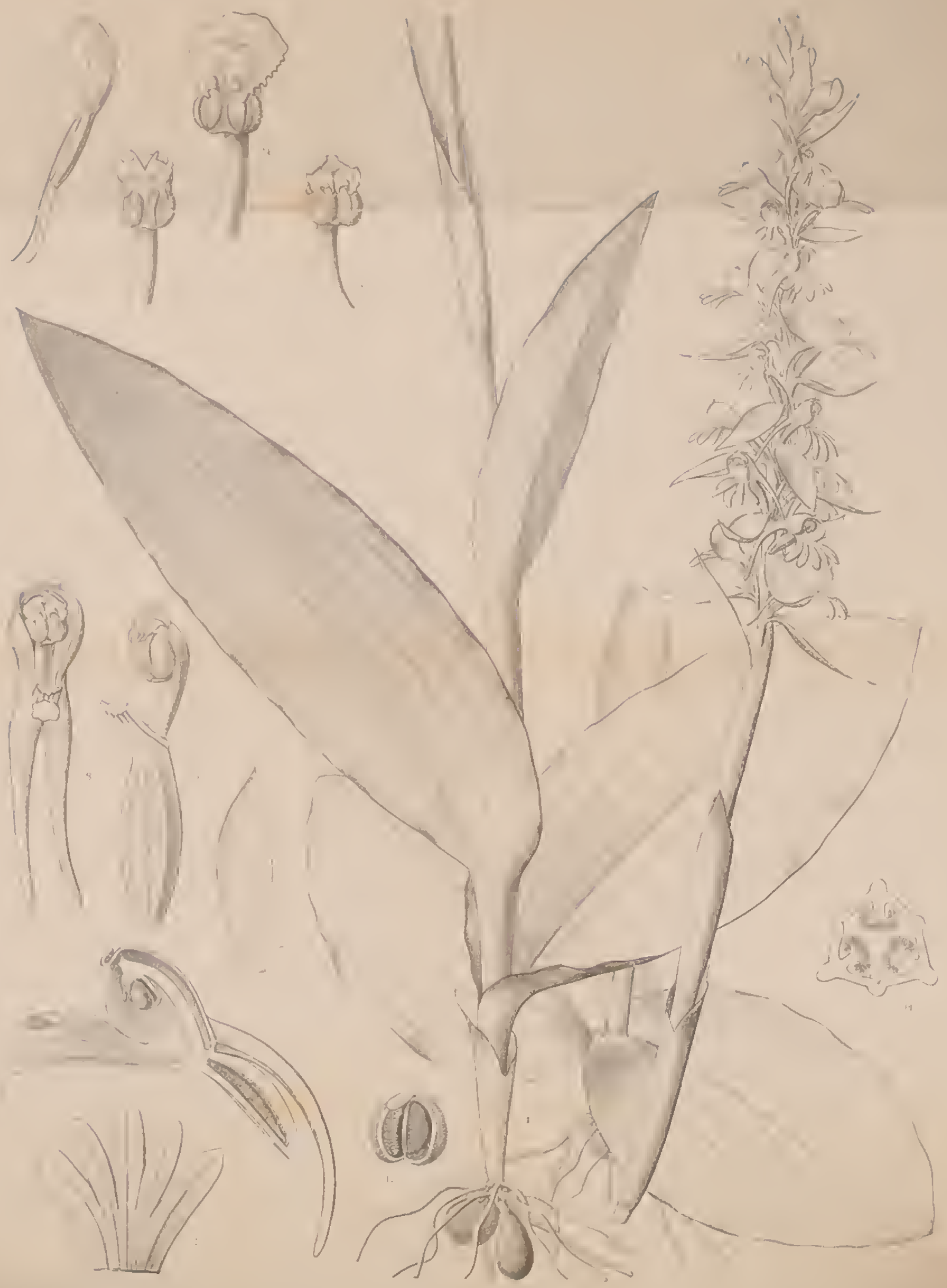




\section{Satyrium morrumbalaensis De Wild. nov. sp.}

Satyrium morrumbalaensis; caule florifero $50 \mathrm{~cm}$ circ. longo, erecto; foliis caulinis ellipticis plus minus vaginantibus, acutis vel subacutis; foliis radicalibus plus minus distinctis saepe binis, ovatis; floribus spicain elongatam densam bracteosam effor mantibus; bracteis lanceolatis, acutis llores majoribus patulis vel reflexis, foliaceis, viridibus, basilaribus cir'c. $2,8 \mathrm{~cm}$. longis; labello galeato, apiculato, calcaribus recurvis apice obtusis, ovarium non aequantibus, majoribus; tepalis inter se coalitis, lateralibus oblongis, subobtusis, supremo lateralibusque aequilongis, glabris, subotusis, gynostemio cylindrici, stigmatis labio erecto. rostello margine dissectis; ovarii costis pubescentibus.

Plante terrestre à tige florifère dressée, atteignant $50 \mathrm{~cm}$. de haut, munie à la base de petits bulbes ovoïdes et d'une gaine courtement fendue. Feuilles caulinaires elliptiques plus ou moins engainantes, à limbe atteignant $16 \mathrm{~cm}$. de long et $6 \mathrm{~cm}$. environ de large, aigu ou subaigu, passant insensiblement anx bractées engainantes qui entourent la base de la scape et de là aux bractées florales ; feuilles basilaires parfois très distinctes, subopposées, ovales, de 8 à $9 \mathrm{~cm}$. de long et $5 \mathrm{~cm}$. de large. Épi florifère

\section{Explication des figures de la planche XIV.}

Fir. 1. - Base d'une plante fleurie dont les feuilles basilaires ne sont pas rapprochèeś $(1 / 1)$.

Fig. 2. - Extrémité de la plante dont la base est figurée en 1 (1/1).

Frg. 3. - Base d'une plante dont les feuilles caulinaires inférieures sont subopposées (1/1).

Fig. 4. - Boutori vu de profil $(3 / 1)$.

Fir. 5 - Bractée florale (1/2). 
de 12 a $15 \mathrm{~cm}$. environ de long, à bractées lancéolées, aiguës, de 12 à $18 \mathrm{~mm}$. de long, égalant ou dépassant les fleur's, étalées ou réfléchies, foliacées, vertes. Fleurs à pédicelle de $9 \mathrm{~mm}$. environ de long, à labelle en capuchon de $7 \mathrm{mım}$. environ de long, à bord retourné, apiculé au sommet, se terminant en deux éperons légèrement recourbés, plus longs que le pédicelle ou ovaire, de $1,5 \mathrm{~cm}$. environ de long, subobtus à l'extrémité; sépales et pétales soudés à la base en un limbe profondément 5 -Jobé de 10 à $12 \mathrm{~mm}$. de long à partir du sommet de l'ovaire, lobes latéraux un peu plus larges que les lobes intermédiaires, tous à peu près de la même longueur, subobtus. de 6 à $7 \mathrm{~mm}$. de long et de $1,5 \dot{a} 2 \mathrm{~mm}$. de diamètre. Colonne cylindrique, grêle, recourbée, logée dans le capuchon formé par le labelle, bilobée au sommet, la partie postérieure formant stigmate, la partie antérieure aplatie, plus ou moins développée, irrégulièrement lobulée au sommet, à 4 dents à la base formant le rostellum sous lequel penchent parallèlement les anthères attachées entre les crans du rostellum et formant une tache plus claire. Ovaire à 6 côtes légèrement velues, dont 3 plus proéminentes correspondent aux placentas.

Hab. - Région du Mozambique : Brousse du Morrumbala, a out 1901 (Ed. Luja n. 424).

Observations. - Le genre Satyrium créé par Swartz est, contrairement à plusieur's des genres que nous avons eu à examiner dans cette

Fig. 6. - Sénales et pétales soudés (3/1).

FIG. 7. - Coupe longitudinale de la fleur (3/1).

F1G. 8. - Fleur dont les sépales soudés et l'ovaire ont éte enlevés atin de montrer la disposition de la colonne dans le labelle (3/1).

Frg. 9. - Fleur réduite à l'ovaire, la colonne et ses accessoires (5/1).

FIG. 10. - Colonne vue de face, avant maturité (7/1).

FIG. 11. - Colonne vue de face, anthères déhiscentes (7/1).

Fig. 12. - Colonne avec rostellum très développé (10/1).

Fı́. 13. - Anthère isolée (10/1).

Fig. 14. - Coupe transversale de l'ovaire. 
livraison, mieux représenté dans la flore extratıopicale de l'A frique et cll particulier dans cellc du sud, que dans le centre tropical de ce continent. Il est presque uniquement africain, on compte quelques représentants dans les iles Mascareignes ct un seul dans l'Inde, le Satyrium nepalense J)on., qui tout en se trouvant isolé sur ce continent rappelle totalement les formes africaines.

Nous suivrons également, pour donner les affinités le cette plante, que nous considérons comme nouvelle, la clef analytique proposée par II Rolfe dans la Flora of tropical Africa (vol. VII, p. 263).

Parmi les trente-trois espèces du genre tel que le considère M. Rolfe, quatre possèdent un labelle à deux sacs courts, plus courts que le limbe, et une espèce un labelle sans éperon ni sac.

Dans le premier de ces groupes, auquel appartient incontestablement la plante que nous figurons, un certain nombrc d'espèces sont caractćl'isées par une paire de feuilles basilaires, apprimées contre le sol. Nous avons indiqué dans la description de notre plante que si les feuilles caulinaires du Satyrium morrumbalaensis sont généralement caulinaires, plus ou moins ascendantes, comme elles se présentent dans le deuxième des groupes établis par Stapf, il existe certains pieds qui pour la dispositions des feuilles sont intermédiaires entre les formes à feuilles apprimées sur le sol et à feuilles dressées; nous avons, afin de faire r'essortir ce détail, fait figurer dans le fascicule XIV, fig. 1 et 3 , les deux aspects. Malgré cette différence, nous rangeons cependant la plante figurée dans le second groupe et comme le labelle possède nne seule paire d'éperons, que ceux-ci ne mesurent pas $24 \mathrm{~mm}$. de long, et qu'ils ne sont pas très divergents des pédicelles, mais au contraire nettement parallèles, notre espèce se range dans le groupe renfermant le plus grand nombre d'espèces. Dans ce groupe, M. Rolfe établit trois catégories :

10 Labelle de 4 à 5 nım. de long, plus ou moins charnu;

$2^{\circ}$ Labelle de 5 à $9 \mathrm{~mm}$. de long, plus ou moins membraneux;

$3^{\circ}$ Labelle de $12 \mathrm{~mm}$. de long.

C'est donc, si l'on tient compte de ce caractère, dans le deuxième groupement que derra sc classer notre plante et c'est en effet du S. coriophoroides A. Rich. de la Région nilienne (Tigre, environ de Dochli, Shire, etc.) que notre espèce se rapproche le plus. Cette dernière espèce a été également publiée sous le nom de S. macrostachyum Hochst, et a été figurée dans le " Tentamen Floræ Abyssinicæ " de A. Richard, planche 89. Si l'on compare la planche que nous venons de citer et celle qui accompagne cette description on jugera facilement des différences; elles portent sur l'ampleur des feuilles, plus développées dans la for'me du Morrumbala, sur les lobes latéraux du limbes formé par la soudure des tépales; ces lobes sont obliquement elliptiques chez le $S$. coriophoroides, 
ils sont elliptiques dans notre plante En outre, la colonne prossède des caractères particuliers. Chez le $S$. coriophoroides le stigmate parait très volumineux et le rostellum est prolongé en avant par une pointe dans laquelle s insèrent les connectif's des masses polliniques; chez le $S$. morrumbalaensis, le stigmate est réduit (fig. 7), le rostellum très développé mais non terminé en pointe en avant, il est lobulé et en're les lobes se trouve la masse basilaire des connectifs des masses polliniques; elle est un peu obscur'e dans les figures 10 et 12 qui représentent le rostellum vu de face, arec les anthères pendantes sous lui. 
Pr. NovakHers Hort THENENSIS.

$P_{1} . X V$

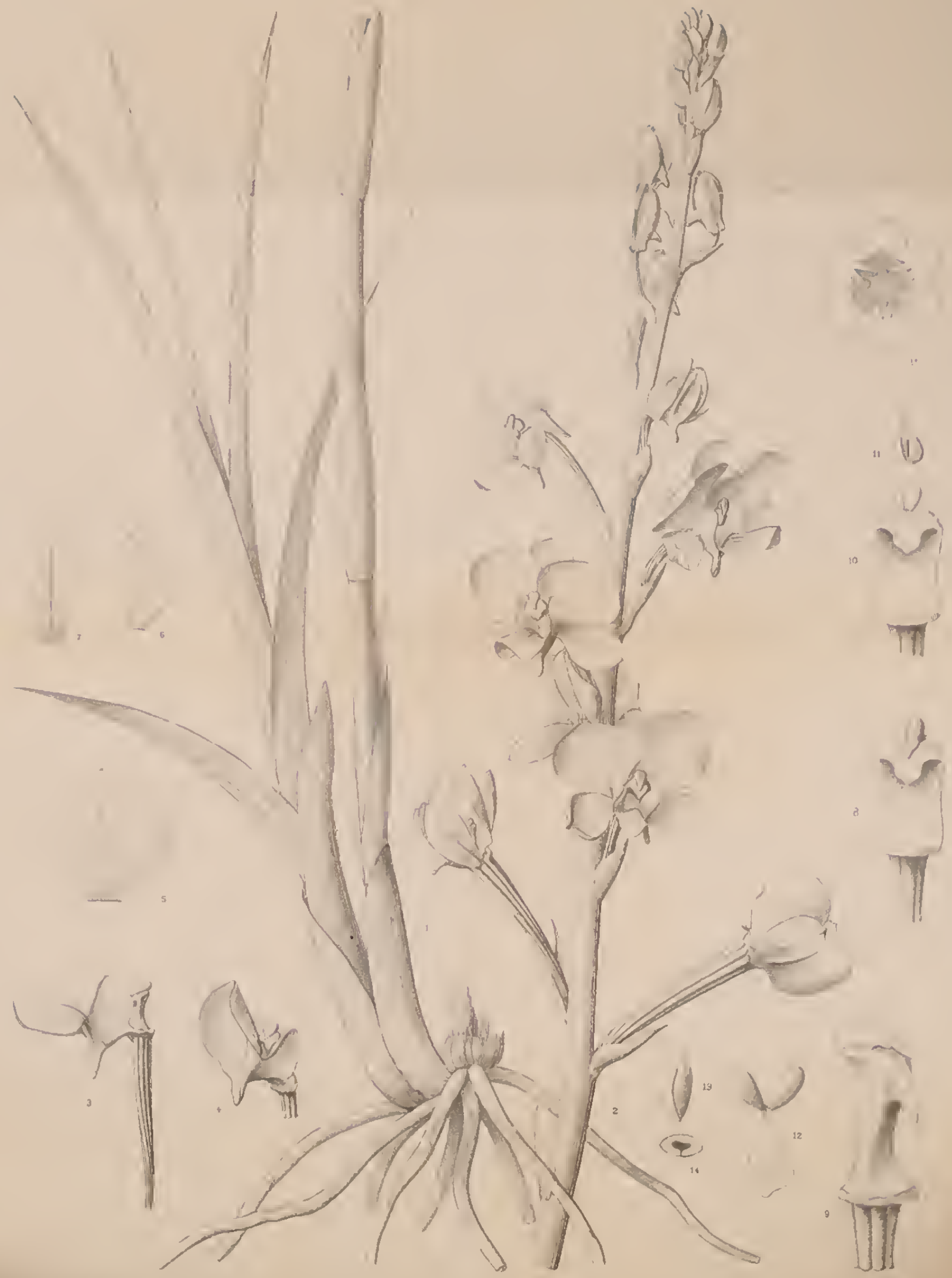




\section{Lissochilus morrumbalaensis De Wild. nor. sp.}

Lisscohilus morrumbalaensis; foliis plicato-venosis, attingente $17 \mathrm{~cm}$. longis et $12 \mathrm{~mm}$. latis; inforescentia non ramosa, bracteis lanceolatis, acuminatis, $2 \mathrm{~cm}$. circ. longis, pedicellos cum ovariis brevioribus; thoribus circ. $3,5 \mathrm{~cm}$. latis, sepalo dorsali obovato, $17 \mathrm{~mm}$. circ. longo, lateralibus oblique-obovalibus, subaequilongis; petalis subrhombeis, $20-22 \mathrm{~mm}$. longis et $20 \mathrm{~mm}$. circ. latis; labelli trilobati, alis rectis oblongis, obtusis, lobo medio rotundato, convexo. carina trilamellata, calcare brevi subobtuso.

Plante terrestre à tige dressée, tiges feuillues et florifères séparées dès la base, naissant sous les vestiges de la pousse précédente remplacée par les fibrilles foliaires, munie de longues racines divergentes. Feuilles basilaires bractéiformes obtuses, entourant par deux les tiges dressées; feuilles caulinaires de la tige stérile, plissées-striées, lancéoléesaiguës, atteignant $17 \mathrm{~cm}$. de long et $12 \mathrm{~mm}$. de large; feuilles des inflorescences bractéiformes, nettement engainantes, au nombre de 3 , atteignant $6 \mathrm{~cm}$. de long. Inflorescence de $22-25 \mathrm{~cm}$. de long, non ramifiée, assez lâche, ¿ bractées lancéolées, acuminées, de 1,5 i $2 \mathrm{~cm}$. de long,

\section{Explication des figures de la planche XV.}

FIG. 1. - Base d'une plante avec tige feuillue et tige florifère (1/1).

Fio. 2. - Extrémité de la hampe fleurie (1/1).

Fig. 3. - Labelle et colonne, vus de profil $(1,5 / 1)$.

Fio 4. - Labelle vu de trois quarts $(1,5 / 1)$.

Fig. 5. - Pétale (1,5/1).

Fıg. 6. - Sépale latéral $(1,5 / 1)$.

Fig. 7. - Sépale dorsal $(1,5 /)$. 
plus courtes que l'ovaire et le pédicelle. Fleurs à pédicelle de $2,5 \mathrm{~cm}$. en viron de long, atteignant $3,5 \mathrm{~cm}$. de diamètre, à sépales verts tachetés de brun; sépale dorsal obovile, courtement apiculé au sommet, de $17 \mathrm{~mm}$. environ de long et $9 \mathrm{~mm}$. environ de large; sépales latéraux obliquement obovales, apiculés, de même longueur environ que le sépale dorsal; pétales jaunes, subcirculaires, de 20 à $22 \mathrm{~mm}$. de long et $20 \mathrm{~mm}$. environ de large. Labelle jaune et brun, trilobé, de $18-20 \mathrm{~mm}$. de long, à lohes latéraux redressés, oblongs, arrondis au sommet, lobe médian arrondi, fortement convexe, à carène à trois crêtes jusqu'à la base, la médiane plus développée que les latérales, à éperon court, subobtus, atteignant environ 4 nim. de long dans sa partie rétrécie. Colonne dressée présentant un appendice recourbé; capuchon à pointe antérieure assez aiguë.

\section{Hab. - Région du Mozambique : Forét de Morrumbala, 15 octo- bre 1900 (Éd. Luja. n. 399).}

Observations. - Le genre Lissochilus R. Brown est représenté uniquement en Afrique et dans les iles Mascareignes. C'est en Afrique tropicale qu'il a surtout atteint son plus grand déreloppement; on y compte plus de cent espèces dont la différenciation est loin d'être toujours aisée, car les auteurs se sont rarement astreints à donner des descriptions comparatives de leur's espèces et à indiquer comment ils comprennent les mensurations des diverses parties de la plante.

En r'evisant les espèces de la Flore de l'Afrique tropicale, M. Rolfe a

FIg 8. - Colonne vue de face (4/1).

Fig. 9. - Colonne vue de profil $(5 / 1)$.

Fı. 10. - Colonne vue de face, le cajuchon qui recouvre les anthères est enlevé (4/1).

Fıg. 11. - Capuchon recouvrant les anthères, vu par dessous (4/1).

FIG. 12. - Anthères sèparées (5/1).

FIG. 13. - Anthère isolée (7/1).

Fig. 14. - Coupe transversale de l'anthère (7/1).

FIG. 15. - Coupe transversale de l'ovaire (15/1). 
constitué parni les quatre-vinght-trois especes qu'il admet, demx grroupes

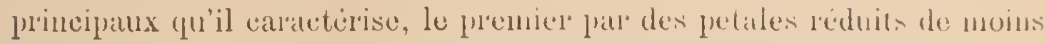
de $10 \mathrm{~mm}$, de long", le second par des pétales d'au nuvins l:2 1111, de long. L'espèce que nous venons de décrire et que nous considérons comne nouvelle appartient donc par ses pétales, de 2 em. environ, très sùrement au second de ees deux groupes qui possède d'ailleur's le plus grand nombre d'espèces.

I)ans la subdivision ainsi eonstitnée, M. Rolfe enploie, pour ponsser. plus loin la clef analytique, les caractères tirés de la forme des pétales; dans son premier groupement il clisse les espèces dont les pétales ront étroits, oblongs ou elliptiques-subspatulés; ce caractère ne correspont pas à notre plante dont les pétales sont largement orbiculaires, caractire que l'on retrouve dans l'énoncé de la seconde subdivision de M. Rolfe : P’étales largement orales-orbiculaires ou orbieulaires-elliptiques. Dans ce groupe se range plus de la moitié des espèces centro-africaines du gremre et, eonnue le ficit renarquer M. Rolfe, il faut encore prendre en considération le $L$. lutus Rolfe, du lac Moero, partiellement connu, et qui a été rangé provisoirenent dans le premier de ees sous-groupes, mais les pétales décrits comme largement subspatulés, de 16 à $18 \mathrm{~mm}$. de long, permettent, semble-t-il, bien que nous n'ayons point vu lit plante, une différeneiation très nette des $L$. latus et $L$. morrumbalcense.

Par son labelle à lobe médian fortement convexe, à eótés reéfléchis, par le disque possédant des crètes longitudinales, des pétales dépassant peu les sépales, un éperon court, droit, subobtus, des fleur's de 3,5 cin. de diamètre pendant l'épanouissement, la plante que nous avons ligur'ée ici appartient à un groupe d'espèces assez nombreuses parmi lesquelles il est difficile de choisir, pour fixer les afhnités de notre plante. 'T'outes ees espèees, si l'on en juge par leurs descriptions, sont trìs semblables. M. Rolfe insiste sur les caractères différentiels suivants :

$1^{\circ}$ Éperon de $4 \mathrm{~nm}$. de long, presque aussi long que les lobes latéraux du labelle;

$2^{\circ}$ Ĺperon de 3 mul. de long, de la moitié ou du tier's de la longuem du labelle;

$3^{\circ}$ Ĺperon de $2 \mathrm{~mm}$, de long, noins du quart de la longueur des lobes du labelle.

Ces earactères sont à premier examen trèr netr, mais si l'on veut bien jeter un coup d'oil sur la figure 3 de notre planche dans laquelle nous avons fait figurer un labelle en plaee vu de profil, on ver'ar (qu'il est tris ditticile de savoir quelle est la proportion à établir entre la longrueur du lobo latéral et de l'éperon par le fiat que la délinitation entro ces éléments floraux n’est pas nettement établie: si l'on consilcre l'éperon 
s'arrêtant an moment oì sa base s'élargit, il nuesurera :3 mm. environ de long et aurait en longueur le tier's de celle du lobe latéral; mais si l'on considère la partie élargie en entonnoir comme appartenan encore à l'éperon, comme l'ont fait certains auteur's, cet éperon serait beaucoup' plus considérable que ne le renseigne M. Rolfe et devrait, d'après le rapport de sa longueur à celle du lobe du labelle, faire passer notre espèce dans le prennier des groupes dont nous avons donné la caractéristique plus haut. D'ailleurs, M. Rulfe lui-mème, dans la deseription du L. crythracae, décrit un éperon de $4 \mathrm{~mm}$., alor's qu'il r'ange cette espèce dans le groupe caractérisé par un éperon de $3 \mathrm{~mm}$; il nous scmble donc que ce caractère est de peu de valeur. Néanmoins, nous consilérons notre plante comme appartenant au second des deux groupes et nous la classerons dans le voisinage des $L$. crythracae Rolfe et $L$. Krelsii Reichb. f., qui se différencient des autres espèces du mème groupe prar des fleur's déveloplúes atteignant $3 \mathrm{~cm}$. de diamètre, celles de notıe plante dépassant même cette mensuration.

Le L. erythraeae Rolfe, spécial semble-t-il à la r'égion nilienne (líolaito plateau, Guido Valley, saganeiti Acrur'), se caractérise par la prósence de pseudobulbes garnis de gaines foliaires ot par des feuilles dont le diametre atteint $3 \mathrm{~cm}$. de lan'ge. (quant au L. Lrelsii Reichb. f., (roisin de notre plante si l'on considère la belle planche qui en a été publiée dans Wrillians Orchid Album, V1, tab. 259, mais distinct du L. Gracfei Kränzlin figuré assez médiocrement dans Reichenbach Tonia Orchidaca, III, 1. 27:2, que .1. Rolfe considère comme synonyme), il en diflère par des feuilles relativenent très étroites, des sépales plus comrts, en outre le disque du labelle ne fos'séderait qu'une erćte jusqu'au delà du milie!ı; notıe $L$. morrumbulaensis comme le $L$. crythraeac en possède trois bien marquées.

La publication des figures de notre espèce permettra peut-être, en présence de nombreux matériaux, de mieux fixer les caractères de.; différentes esjèces encore très embrouillées. 


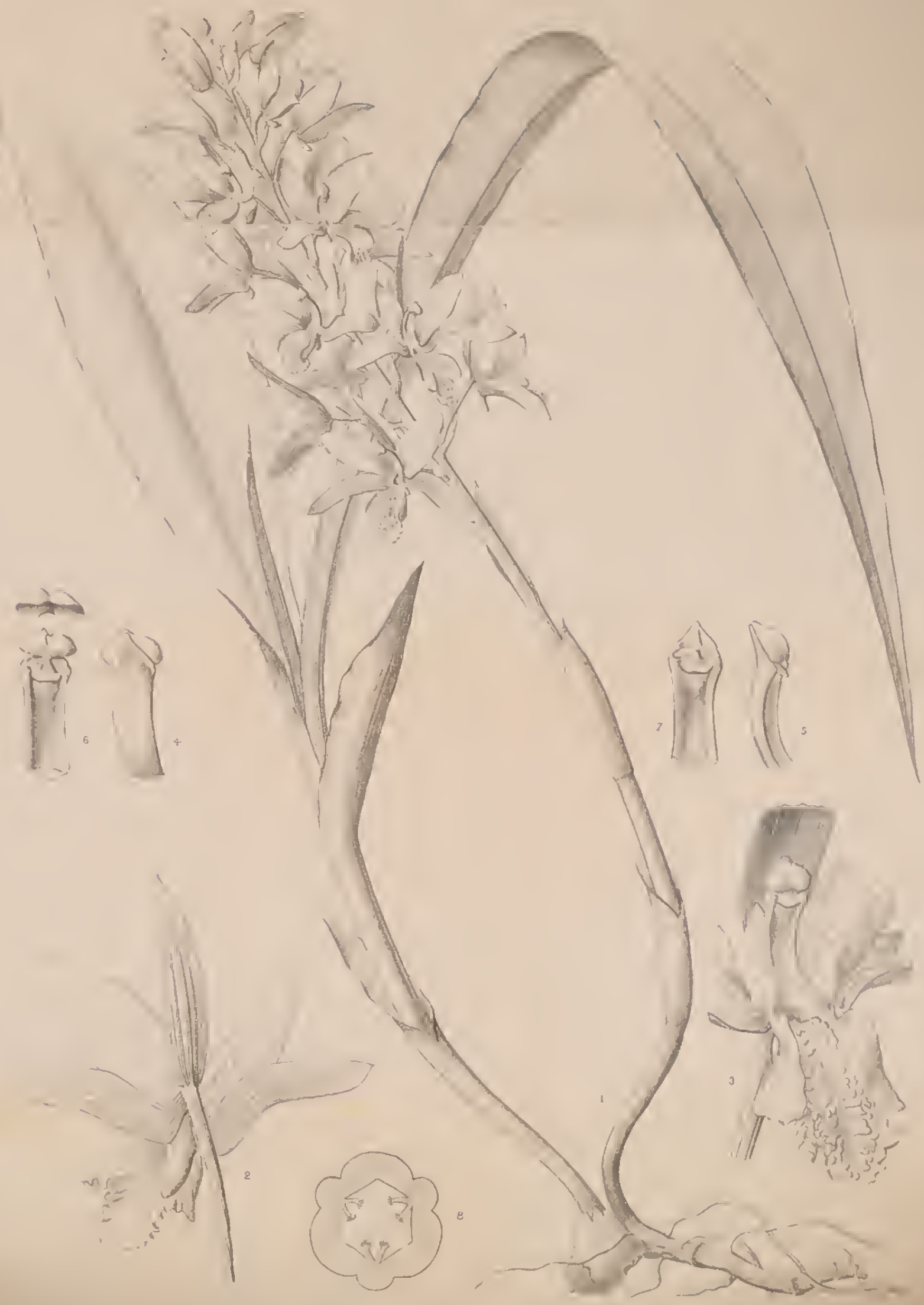




\section{Eulophia Kirkii Rolfe}

in This.-Hyer Fl. trop. Afr. VII (1897) p. 5s.

Plante à rhizome plus on moins épaissi, noneux, à racines fibrilleuses, plus ou moins nombreuses. Fenilles et Heur's sur des tiges diflérentes. Fenilles inférieures engainantes, terminées latéralement par un limbe court, aigu, les suivantes ohlongues ou lancéolées, rétrécies plus ou moins en une sorte de pétiole engainant, canaliculé, atteignant 15 à $36 \mathrm{~cm}$. de long et 2,4 à $3,5 \mathrm{~cm}$. environ de diamètre, acuminées-aiguris, plus on moins plissées. Tige fleurie atțeignant:30 cm. de long, munie uniquement de gaines et de bractées engainantes à la base; les bractées de la tige passant insensillement aux bractées florales. Racèmes de 13 à $20 \mathrm{~cm}$. de long, multiflores, fleurs diversement colorées, à bractées linéaires, lancéolées, aiguës, aussi longues que les pédicelles, de 12 à $20 \mathrm{~mm}$. de long, membraneuses et plus ou moins blanchaitres-scarieuses. Pédicelles de 10 à $16 \mathrm{~mm}$. de long. Sépale postérieur oblong-aigu, à nervation marquée sur le dos, de 18 à $23 \mathrm{~mm}$. de long; sépales latéraux un pen plus longs que le terminal, aigus, légèrement arqués à la base, à nervures apparentes. Pétales

\section{Explication des figures de la planche XVI.}

Fic. 1. - Plante grandeur naturelle.

Fia. 2. - Fleur vue de dos(2/1).

Fis. 3. - Labelle et colonne, vus de face $(4 / 1)$.

Firs. 4. - Colonne vue de dos $(4,1)$.

Fia. 5. - Colonne vue de profil (5/1). 
ovales-oblongs, aigus, nembraneux, de 14 a $17 \mathrm{~mm}$. de long et atteignant 7 : $8 \mathrm{~mm}$. de diamètre. Lahelle trilobé de $14 \mathrm{~mm}$. environ de long, à lobes latéranx oblongs, arrondis au sommet, olitus, redressés; lobe médian suborbiculaire, ondulé sur les hords, à côtes verruquenses-crénolées, proéminentes sur la partie antérieure. Éperon relativement court, conique-oblong, de $4 \mathrm{~mm}$. environ de long, subobtus. Colonne suhclaviforme de 6 à $8 \mathrm{~mm}$. de long, canaliculée. Ovaire à côtes obtuses, placentas bifurqués opposés aux côtes étroites.

Hab. - District du Mozambique Manganja Hill: Shire Highlands ; montagnes de Morrumbala (entre 1.500 et 3.500 pieds, Kirk et 25 décembre 1900, Luja, n. 396).

Observations. - Le genre Eulophia est représenté dans la flore du monde par environ deux eents espèces. Il est l'eprésenté dans toutes les r’égions tropicales du globe, mais les espèees sont partieulièrement abondantes en Afrique et deviennent beaueoup plus rares dans les Indes, la Polynécie, l'Australie et l'Amérique tropicale.

I a elassifieation de ee genre et son rénombrement en sous-genres est. loin d'être uniforme; la plupart des auteurs qui ont étudié les espèces qui le eomposent arrivent i des eonchusions différentes. Nous n'arons pas à entrer ici dans la discussion de leur manière de roir, et eiterons uniquement pour exemple de cette divergenee d'opinion la eréation. par M. le professeur Pfitzer, de Heidelherg, du genre Eulophidium pour l'E. maculatum Reichb. f.. genre que II. Rolfe ne conserve pas, à tort nous semble-t-il.

Comme le fait ressortir Mr. Rolfe, dans la Flora of tropical Ifriea, quelques espèces du genre Lulophic forment une réritalle transition vers le genre Lissochilus, dont nous avons rappelé les caractères plus haut à propos du Lissortilus morrumbalacnsis, déerit à la suite de la planehe XV.

Parmi les nombreuses espèees de ce grenre existant dans le centre africain, l'E. Kirkii forme avec les $E$. cuglossa Reielıb. f. (SierraLeone, Sugar-loaf Mountain, Old ('alahar, Ingola), E. Kirkii Rolfe et

Fis. 6. - Colonne rue de face, capuchon enlevé $(4 / 1)$.

Fıg. 7. - Colonne vue de face, masses polliniques enlevées (4/1).

Hig. 8. - Coupe transversale de l'ovaire (25/1). 
E. stuchyorles Reiehb. f. (Niam-Niam et Mombuttu), un groupo caractérisé par les tiges à plusieur's feuilles atténuées à la base, des flenrs ¿ éperon oblong lisposées en l'aeème assez grand, it sépales de 14 à 24 mm. de long et des braetées environ aussi longues que les pédicelles flomanx.

De ces trois dernières espèees l'Fe euglossa est la seule dont les lobes latéraux du labelle sont aigus, les deux autres possèdent un labelle à lobes latéraux obtus, comine on peut le voir dans les figures I et 3 de notre planche. Chez l'Eulophia stachyodes les feuilles apparaissent après les flour's, tandis que ehez l'E. Kirkii feuilles et fleur's sont eontemporaines.

Cette espèce, déerite depuis peu, n’avait pas été figurée; pour fixer' davantage ses earactères, nous l'avons fait dessiner. les échantillons rapportés par M. Lıija de son séjour sur le Morrumbala étant en très bon état de conservation. 



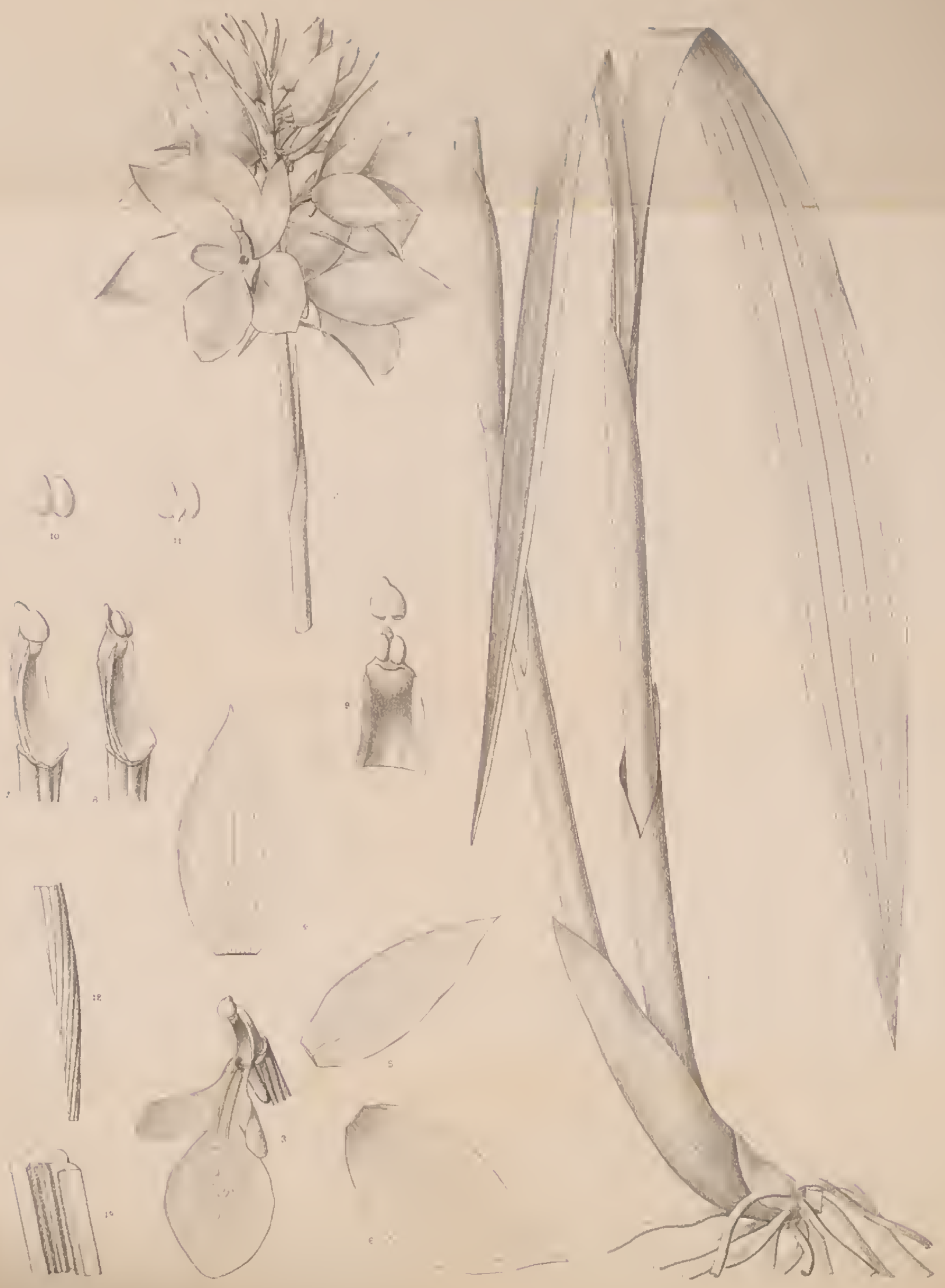




\section{Eulophia Lujae De ITild. nov. sp.}

Eulophia Lujae; caulc $30-10 \mathrm{~cm}$. longi, vaginis laxis, oblongis, sulracutis; vestitu foliis radicalibus juxtacaulcm floriferum, vaginis inferum binis plus minus distantibus, foliis oblanceolatis, acutis, plicatis, non maturis cire. 2,5 cm. latis, basi angustatis et in petiolmm desinentibus; lloribus pedunculatis racemum simplicem terminalem cire. $8 \mathrm{~cm}$. longum efformantibus; bracteis lanceolat.s accutissimis orariun cum pedicello longioribus; sepalis ovali-oblongis acutis, subacqualibus, petalis ovali-oblongis acutis brevioribus; labello basi breviter nuguiculato, ungue in calcar breve, rectum desincute; lamina triloba, lobis lateralibus obtusis, terminali longiore, obtusa, basi bicarinata; grnosteg.o clongato, concavo; pollinis 2 ovoideis postici in caudicula communi angusta.

Plante terrestre atteignant 30 et $40 \mathrm{~cm}$. de hant, it souche plus ou moins épaisse, gannie de racines filreuses, à tiges fleuries et feuillues séparées dès la base, les fenilles atteignant leur complet développenent après les lleur's ; base des deux tiges entomée par une ou deux bractées engrainantes. 'l'ige feuillue à 2 ou 4 gaines d'oin sortent ¿ feuilles oblancéolées-aignës, plissées, atteignant, non adultes, $30 \mathrm{~cm}$. de long et jusque $2, \overline{\mathrm{cm}}$. de large; tige llorifëre à gaines passant aux bractées. Fleurs disprosées en une

\section{Explication des figures de la planche XVII.}

Fu 1. - Plan.e grandeur naturelle.

Fu: 2. - Extrémitè de la tige lleurie (1/1

Frs. 3. - Labelle et gynoslège $(1,5 / 1)$.

Fiu. 4. - Sépale postérieur $(1,5 / 1)$.

Fis. 5, - Pélale $(1,5 / 1)$. 
inflorescente drnse, à plus do o lleur's pédonculées, jaunes. de $6 \mathrm{~cm}$. environ de diamètre, formant un lasème ternninal, simple, de $8 \mathrm{~cm}$. environ de long et de $8 \mathrm{~cm}$. de lar'ge; bractées lancéolées-aiguës, de 1, 5 à $2,5 \mathrm{~cm}$. de long, plus longues que le pédicelle et l'ovaire mesurant environ I à $1,5 \mathrm{~cm}$. de long. Sépale postérienr ovale-aigu, sépales latéraux légèrement ohliques, ovales, aignts, un peu plus larges que les sépales postérieurs, de $3 \mathrm{~cm}$. environ de long sul' 1,5 cm. de large; labelle trilobé de 2,-5 ('nu. environ de lonš, légèrenent onguiculé à la lase, à onglet se prolongeant en mu épreron contut, droit, obtus, de 4 a j mm. le long, lobes latéranx étalés-dressés, arrondis au sommet, lobe mérian ovale, olıtıs. an sommet, muni de poils sur sa partie centrale et à sa base de deux carrnes nettement proéninentes. Colonne allongée, concave, de 9-10 mm. de long, capuchon aigu, pollinies ovö̈des, réunies par paire par' un candicule commun court et postérieur.

Hab. - Région du Mozambique : Dans la brousse, Morrumbala. novembre et décembre 1900 (Éd. Luja, n. 391).

Obscrvations. - Dans les observations qui accompagnent la description de l'Euloplia Kirkii nous avons fait ressortir dans leurs grandes lignes la distribution de ce genre dans le monde et sa subdivision en groupes plus ou moins naturels. Par l'ensemble de ses caractères la plante que nous venons de décrire apparlient au mème groupement primordial que l'E. Kirkii, mais par' son inflorescence plus dense l'E. Lujae

F19. 6. - Sépale latéral $(1,5 / 1)$.

Fia. 7. - Colonue, surmontée des pollinies recouvertes par le capuchou, vue de protil $(3 / 1)$.

FIG. 8. - Colonne, surmontée des pollinies capuchon enlevé, vue de protil $(3 / 1)$.

Fis 9. - Fragment de la colonne vu ile face, le capuchon recouvrant les pollinies dètaché $(3 / 1)$

Fig. 10. - Pollinies vues de face $(5 / 1)$.

Fia. 11. - Pollinies rues de dos (5/1)

Fiti. 12. - Ovaire (3/1).

FIa. 13. - Coupe longitudinale d'une portion le l'oraire $\left(S_{1}^{\prime} 1\right)$. 


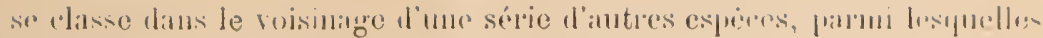

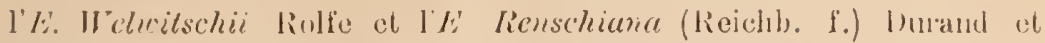
schin\%, do l'Angola, paraissent avoir eertaines aftinités. Les calacteres qui nous amònent à opérer ce classement sont : le dévelrplement complet des feuilles après eelui des scipes, eomne nons l'avons fait r'enarquer dans la dercription, l'étroitesse r'elative des sépales. surtout dı sépale postérieur, la longueur de l'éperon et lı villosité du labclle.

M. Rolfe, dans la l'lora of tropical Ifrica, diflérencie les deu expéces que nous venons le eiter par les caraetères :

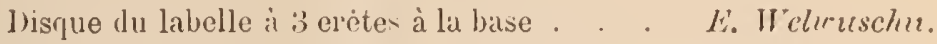

Disque du labelle à 2 crètes à la base . . L. Renschiana.

Comme le fait nettenent r'essortip la figure 3 de notre planelie, le labelle possède à la base deux eretes très distinetes. Ce serait done de la seennde de ees denx espèees que se lapproeherait surtout notre plante nouvelle. Malheureusement la description de l'F. Renschiana (=Orthochilus Renschianus Reichb. f. in Flora 188: 1). 532) est très éeourtée; l'auteur n'en a point déerit les sépales ni les pétales et donne d'une façon très: sommaire les caractères du labelle qui est, d'après lui, obtus ou émarginé; ee dernier caraetire ne enneorde pas du tout avee relui que l'on observe dans la figure 3 de notre planche. M. Rolfe déerit l'éperon eoinme grèle, aigu, très eourt, r'ecourlyé; or', dans notre plante l'èperon est obtus et non recourbé.

D'un autre eôté on peut également supposer des affinités de notre plante avee l'E. milanjana Rendle qui appartient lui à la flore du Mozambique (nous ne eonnaissons, il est vrai, eette plante que par sa deseription) et qui se earactérise par ses raeèmes denses presque en corymbe. C'est sur la densité des infloreseenees que M. Rolfe base la différeneiation des groupes; malheureusement ee earaetere est soumis à de multiples variations suivant l'appréeiation des observateur's. M. Rendle déerit les fleurs eomme jaunes, à lobes latéraux du labelle bleus; eette eoloration n'est pas signalée par' M. Luja, en outre l'auteur du l'E. milanjana déerit un éperon eonique et une enlonne de à mm.; dans l' 2. . Lujcue, l'éperon est eylindrique, obtus et la eolonne mesure de 8 à $10 \mathrm{~mm}$, de long. 




\section{Polystachya Lujae I)e Wild. nov. sp.}

Polystachya Lujae; pseudobulbis lineari-oblongis, $11-12,5 \mathrm{~cm}$. longis; folia unica, oblonga, cuneata-subicuta, $12.13 \mathrm{~cm}$. longa et 2,5-3 cm. lata, glaberima, scapo subaequilonga. Scapus terminalis, subracemosus, bracteis parvis, acuminatis, spica $5 \mathrm{~cm}$, circ. longa, cum floribus glabrescens. Perianthium erectum, glabrum, ovario subaequilongo.

Plante épiphyte à rhizome très court, noircissant par la dessiccation; psendobulbes linéaires-oblongs, de 11 ì $12,5 \mathrm{~cm}$. de long, glabres, munis de 2 ou 3 bractées engainantes, laissant après la clute une trace circulaire sur le pseudobulbe. lieuille unique, oblongue, cunéiforme, subaiguë au sommet, embrassinte à la base, de 12 à $13 \mathrm{~cm}$. de long et 2,5 à $3 \mathrm{~cm}$. de large, glabre, coriace. Scape de 11 à $13 \mathrm{~cm}$. de long, aussi longue environ que les feuilles et glabre comme elles, entourée à la base par 1 ou 2 longnes braetées, fortement engainantes, assez rapidement caduques; panicules de $5 \mathrm{~cm}$. environ de long, plus ou moins ranifiées, à bractées triangulaires, ovales, assez brusquement acuminies an sommet, aignës, de $5-6 \mathrm{~mm}$.

Explication des figures de la planche XVIII.

Fía. 1. - Plante en fleurs et en fruits $(1 / 1)$

Fro. 2. - Jeune fruit (4/1).

Fia. 3. - Fleur épanouie avec sa bractée $(4 / 1)$.

Fra. 4 - Fleur épanouie (4/1).

Fro. 5. - Fleur épanouie, sẹpales latéraux enlevés (4/1)

Fra. 6. - Fleur dont les sépales et un pétale ont été enlevés 8/11.

Fıa. 7. - Sépale latéral, vu par la face inlerne (4/1). 
de long. Pédicelle de $5 \mathrm{~mm}$. environ de long, grêle, glabre. Fleurs blanches à sépale dorsal ovale-triangulaire, aigu, de $6 \mathrm{~mm}$. environ de long, sépales latéraux aussi longs yue le postérieur, aigus, carénés. Pétales elliptiques de $5 \mathrm{~mm}$. environ de long. Labelle en fer de lance non trilobé, longuement aigu, rétréci à la base, le $6 \mathrm{~mm}$. de long, à disque pubérulent-velu. Colonne courte, de $1,5 \mathrm{~mm}$. environ de long, concave, à ailes latérales peu proéminentes, arrondies all sonmet; distance du sommet de l'ovaire à la base du labelle de 3 à $4 \mathrm{~mm}$. environ. Ovaire a 6 còtes, les plus lirges correspondant aux placentas internes.

Hab. - Région de Mozambique : Forêts du Morrumbala, février, 1901 (Ed. Luja. n. 377).

Obserrations. - Le genre Polystachya renferme actucllement une centaine d'cspèces et les recherches qui s'eflcctucnt journellement en Afrique vont encore augmenter ce nombre, car ce genre est certes un des plus polymorplies parmi les Urchidécs; malleureusement pour ce genrc cumme pour presque toutes les olchidées africaines il manque dans beaucoup d'herbier's des matériaux de comparaison et l'on est amené à faire de fréquentes erreur's, quand il faut se baser uniquement sur les descriptions.

Dans la Flora of tropical Africa que nous arons citée à propos de chaquc espèce de ce fascicule, M. Rolfe a subdivisé le genre en se basant sur la disposition des psendobubes et a créé les groupements :

Pseudobulbes ou tiges rapprochées ou en touffes.

Pseudobulbes ou tignes grèles, superposés.

Pscudobulbes ovoides, plus ou moins distants le long d'un rhizome ramplant.

Si nous nous basons uniquement sur ces courtes diagnoses, nous classerons la plante que nous venons de figurer dans le premier des groupes,

F1s. 8. - Sépale dorsal, vu par la face interne (4/1).

F1G. 9. - Pétale (4/1).

Fig. 10. - Colonne, vue de face $\$ / 1)$.

Fig. 11. - Colonne, vue de face, capuchon recourrant les anthères soulevé (8/1).

F16. 12. - Coupe transversale de lovaire $(8 ; 1)$. 
car il ne pent appartenir au seconul. les pseudolulhes n'étant pas superposés, ni au troisième, les pseudobulbes étant linéaires et non ovoüdes. Cependant, il y a lieu de faire remarquer qu'au point de vue de la disposition des pseudobulbes, les raractères de la troisième section correspondent bien à notre plante telle qu'elle est figurée figure 1 de lit planche XVII, e'est-ì-dire que les pseudobulhes sont alignés sur un rhizome tracant.

Par tous ses autres caractères : rachis do l'inflorescence, granleur des flemrs, distance de l'ovaire à la base du labelle. forme du labelle, et par ses feuilles solitaires ohlongues, le $P$. Lujae est très affine de $P$. cultrafr Lindl. décrit et fignré par' Thouars dans ses Orchidées des îles africaines, t. 87, sous le nom de Dendrobium cultriforme.

Si l'on compare la planche que nous itonnons ci-contre avec celle do l'ouvrage de Thouars, nous remarquerons une première différence : c'est la longueur relativement beaucoup plus grande du pseudobulbe dans li phante de M. Luja; pour les feuilles les mensurations sont à peu près hess mêmes; tandis que chez le type de Thouars provenant de Maurice, Madagascar et Bourhon, les feuilles sont deux fois environ aussi longues que les pseudobulbes, dans la plante du Zambèze pseudubulbes et feuilles sont ì peu près de même longueur.

('e caractère est, il est vrai, mitigé si l'on consulte la .. Flora of tropical Africa " qui décrit les pseudobulbes de cette espèce comme variant de $8 \mathrm{~mm}$. à $10 \mathrm{~cm}$. de long, et les feuilles de 6 à $14.5 \mathrm{~cm}$. de long: ces dernières sont done geénéralement plus longues scmble-t-il que les pseutobulbes. Certaines mensurations des fleurs ne concordent également pas. les fleurs de notre $P$. Lujue paraissent plus développées. Nous arons donc tenu à figurer cette plante. car' $\mathrm{l}$ était bon de fixel'sur elle l'attention. afun de déterminer si la plante des iles Manrice, Madagascar et Bourhon est bien la mème que celle signalée au Cameroun, dans l'Usambar’d et dans le Marangu, et par suite si la plante que nous décrirons ici m. devrait pas ètre considérée comme le type auquel derraient ètre rapportées les plantes voisines appartenant à la flore de l'Afrique continentale. 


Pl.Novae Herb. Hort Thenensis.

PL. XIX
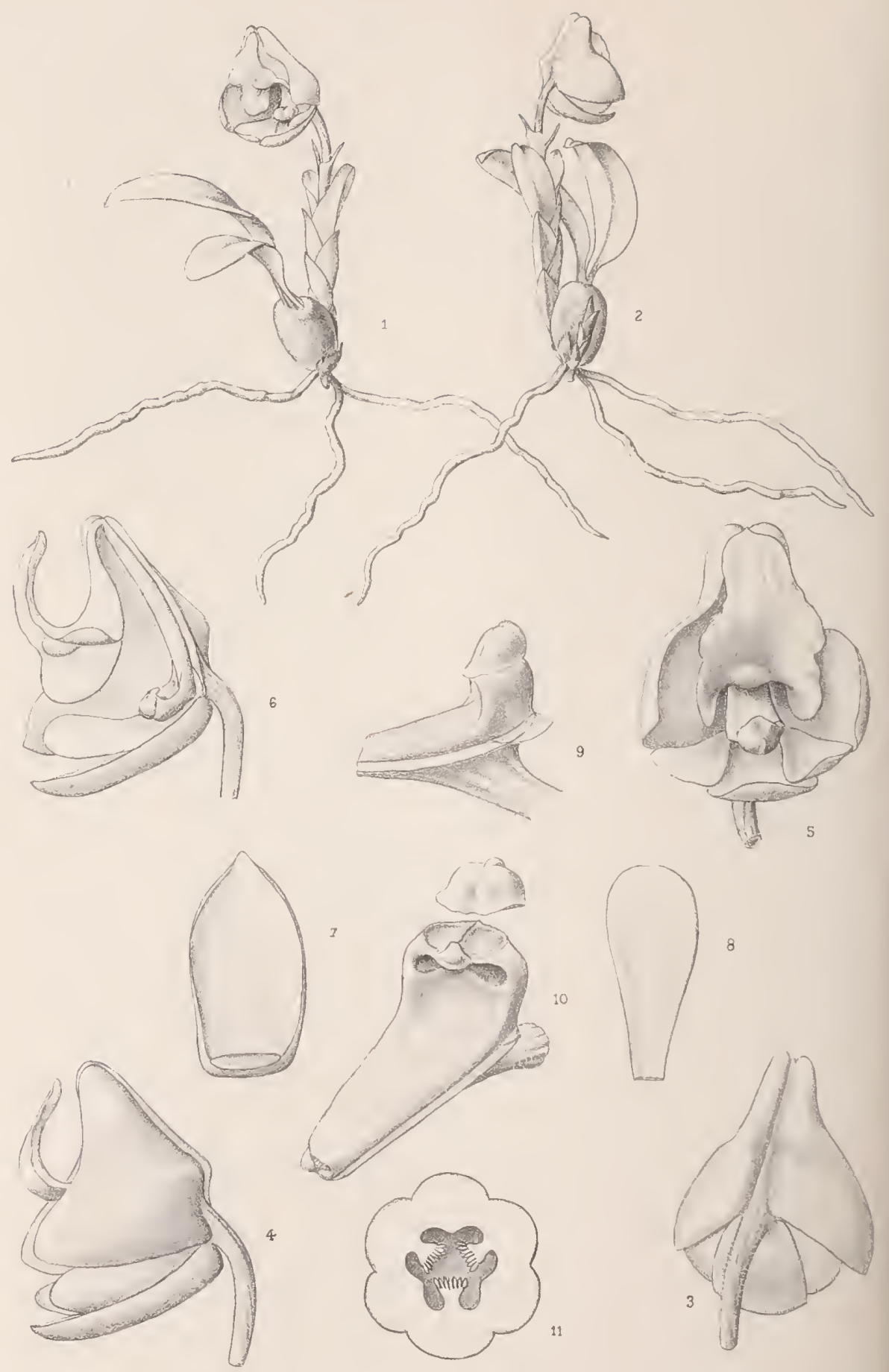

A.d'Apreval, ad nat del

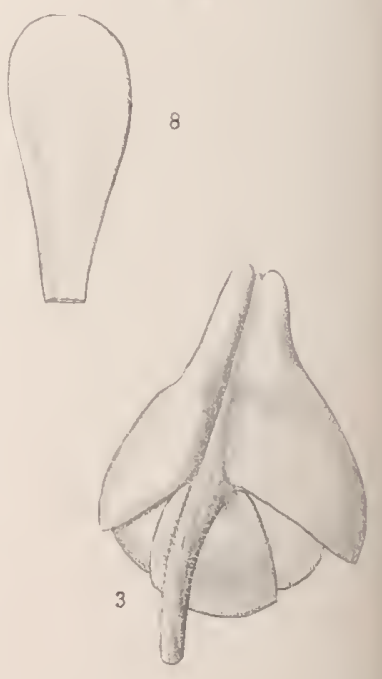

Imp. J Minot, Paris

POLYSTAGHYA UNIFLORA De Wild. 


\section{Polystachya uniflora De Wild. nov. sp.}

Polystachya uniflora; pseudobulbis parvis, 2-phyllis, foliis lineari-oblongis, carnosis, $2-3,5 \mathrm{~cm}$. longis, pedunculo basilari unifloro, dense bracteato, bracteis basilaribus carinatis, bracteis superioribus abrupte acuminatis, flore albo, sepalis subobtusis, lateralibus inajoribus, petalis $9 \mathrm{~mm}$. circ. longis, oblongo-spathulatis, obtuso-rotundatis, labello carnoso trilobo, lobo intermedio subobtuso, lobis lateralibus rotundatis, erectis; columna lata, brevi.

Plante réduite, à pseudobulbes subaplatis de 10 à $11 \mathrm{~mm}$. de long, à angles latéraux subaigus, munis de racines grêles, et munis au sommet de deux feuilles de $2: 3, \overline{5} \mathrm{~cm}$. de long et atteignant $8 \mathrm{~mm}$. environ de large, d'un bean vert, charnues. Inflorescences basilaires, partant de la base du pseudobulhe, solitaire ou par deux, ì une seule tleur terminale, à pédoncule de $3 \mathrm{~cm}$. de long, densément hrätéolé, à bractées carénées, nettement imhriquées, les inférieures arrondies au sommet, les supérieures heaucoup) plus réduites, brusquement acuminées au sommet. Péllicelle constitué par l'ovaire, d'environ l cm. de long,

\section{Explication des figures de la planche XIX.}

Fıci 1. - Plante entière avec une hampe florale (1/1).

Fıc. 2. - Plante entière, une liampe florale développée, une hampe Horale jeune (1/1)

Fı́. 3. - Fleur épanouie, vue de dos $(3 / 1)$.

Fig. 4. - Fleur épanouie, vue de profil (3/1).

Fı. 5. - Fleur épanouie, vue de face (3/1).

Fıo. 6 - Fleur vue le profil, un sépale latéral enlevé $(3 / 1)$. 
courtement velu. Fleur blanc-crène, de $1,5 \mathrm{~cm}$. environ de diamètre, ì sépale postérieur oblong, subaigu, les latéraux largement triangulaires, de mème longueur environ que le sćpale dorsal, mais plus larges, subobtus, de $9-10 \mathrm{~mm}$. de long; pétales de $9 \mathrm{~mm}$. environ de long, ohlongs-spatulés, obtus an sommet; labelle charnu, replié, ondulé sur les bords, trilobé, à lobe médian ovale, à lobes latéraux redressés, arrondis, disque relevé au centre. Colonne droite, conte, obtuse, a bords arromdis. Histance du sommet de lovaire a l'insertion du labelle de l cm. environ.

Hab. - Région de Mozambique : Morrumbala 1901 (Éd. Luja, s. n.).

Observations. - Cette petite plante nous a par'u particulièrement curieuse; nous lui avons donné le nom spéeifique uniflora en nous basant sur le fait que la lrampe florale est terminée par une seule lleur; nous tenons eependant à faire remarquer immédiatement que parmi les nombreux Polystachya elle nest pas seule à posséder des hampes florales uniflor'es; nous pouvons eiter entre autres le P.grandifiora Lindl. (Bot. Mag. t. 3707).

Si lon tient eompte des données de la Flora of tropieal Afriea, où M. Rolfe a, eomme nous l'avons vu précédemment, essayé le elassement des espèees afrieaines elı se basant sur les pseudobulbes, il faudra ranger' la petite plante nouvelle dans le voisinage du $I^{\prime}$. caespitifica líänzl. originaire d'une région similaire (Afrique orientale allemande, à Mbaramu sur' les trones d'arbres dans la forêt vierge où elle a été réeoltée par Holst).

Mais la différeneiation entr'e ees deux espejees est facile à établir, ear la plante de l'Afrique allemande possède des pseudobulbes à une seule feuille et eelle-ei est linéaire; on outre, la fleur' ne mesure que $4 \mathrm{~mm}$. de diamètre, elle est donc beaueoup plus petite que eelle de notre plante, qui pour un Polystachya est relativement très développée.

Fig. 7. - Sépale dorsal (31).

I'ıu. 8. - Pèlale (3/1).

Fia. 9. - Colonne vue de profil $(5,1)$.

Fı. 10. - Colonne se prolongeant jusqu'au labelle qui a été enlevé $(5,1)$.

Fio. 11. - Coupe trausversale de l'ovaire, fortement grossie. 

Pl Novae Herb. Hort. Thenensis.
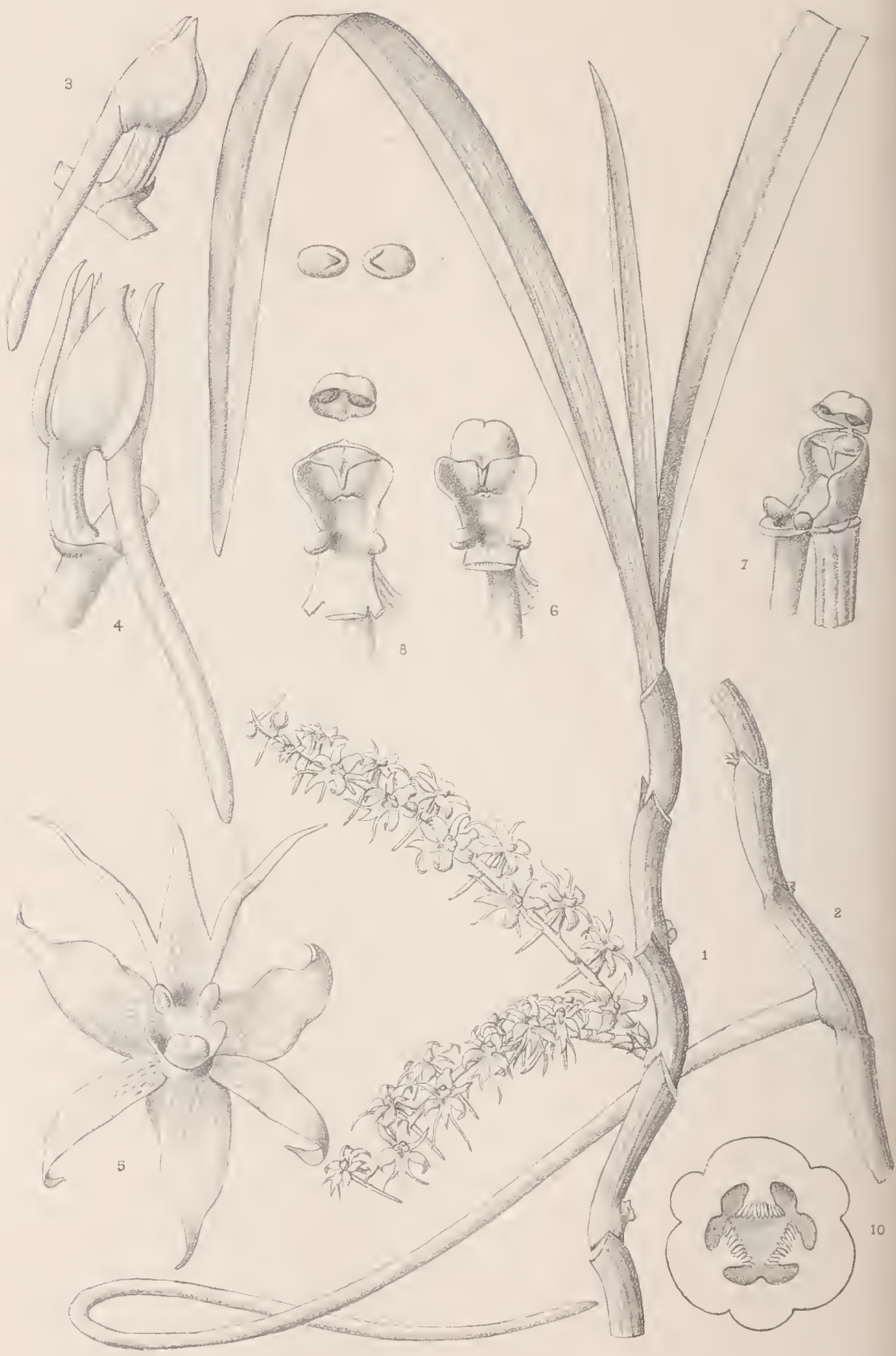

AdApreval.ad nat del imp.i.Mino Paris ANGPAECUM TRIDACTYLITES RoIFe 


\section{Angraecum tridactylites Rolfe}

in Gardn. Chronicle IV (1888) p. 34 et in This.-Dyer Flora trop. Afr. VII p. 1.17.

Angraecum armeniacum Reichh. f. in Flora (1S65) p. 189.

Plante épiphyte, à tiges dressées, grimpantes, atteignant (i) cm. de long, munie le long de la tige aplatie de racines fortes et assez longues qui percent le dos des gaines folianires. Feuilles allonğées-linéaires, lég’èrement et inégarlement birlentées au sommet, de 9 à $20 \mathrm{~cm}$. de long et 8 i $14 \mathrm{~mm}$. de large. Racèmes pluritlores, axillaires, perçant liı gaine des feuilles, solitaires ou géminées, de 4 à $8 \mathrm{~cm}$. de lonğ; tleurs brunâtres, disposées d'un seul côté du l'ilchis, de $8 \mathrm{~mm}$. environ de diamètre. I'édicelle de $4 \mathrm{~mm}$. environ de longr, muni à la base d'une bractée courte entourant la tige d'un liseré. Sépales subégaux, ovales-acıminés, le sépale dorsal parfois un peu plus étroit que les latéraux, de $4 \mathrm{~mm}$. environ de long. Pétales lancéolés-

\section{Explication des figures de la planche XX.}

Flg. 1. - Rameau tlorifère (1/1).

Fı, 2 - Base de ce rameau, avec une racine latérale perçant la gaine forliaire $(1)$

Fir. 3. - Bouton (6/1).

Flu. 4. - Bouton peu arant l'épanouissement (b/1).

Fio. 5. - Fleur épanouie vue de face $(5 / 1)$.

Fus. U. - Colonne avec anthères recouvertes d'un capuchon $(8,1)$. 
linéaires, aigus, environ anssi lonųs que les sépales. Labelle trifurąué, de 4 mm. environ de long, à lobes listéraux un pen plus longs et plus grèles que le lobe médian, celuici triangulaire, aigu, rigide; de chaque côté de la base du labelle se trouve un appendice marginal, dentiforme, charnu. Éperon grêle atteignant $10 \mathrm{~mm}$. de long, plus ou moins pointu à son extrémité. Colonne assez épaisse, de 1,5 mm. environ de long, ailée latéralement, à ailes arrondies.

Hab. - Guinée supérieure Sierra-Leone, Lagos); Guinée inférieure Angola, ile des Princes: Nyassaland. A cette distribution il faut ajouter : Région de Mozambique : Morrumbala, novembre et décembre 1900 (Éd. Luja, n 390).

Olservations. - Cette jolie espèce a été décrite en 1888 par M. A. Rolfe, sur un échantillon qui avait été envoyé de sierra-Leone à Kew jar M. 'T.-I3. Griffith, secrétaire colonial de Sierra-Leone. Sans posséder la valeur horticole de certaines Orchidées américaines, cet $A n$ graecum, dont la distribution parait assez vaste en Africue tropicalo puisqu'il existe en Afrique tropicale orientale ou il a été recueilli dans le Nyasaland par Buchanan, et dans l'Afrique oecidentale, mériterait d ètre cultivé dans les serres européennes oì il tiendrait une place honorable, parmi ses congénères, gràce surtout à sa floraison abondante et à la durée de ses lleurs.

Le genre Angraccum est très largement représenté dans la flore de l'Afrique tropicale; la flore de cette région, publièe sous la direction de sir W. Thiselton-1)yer, signale quarante-luit espèces, auxquelles il faut ajouter uno série assez nombreuse d'espèces dierites depuis 1898, date de lapparition de cette partie du trarail de M. Rolfe. Le greme Angraccum est d'ailleur's un des genr'es les plus richement repr'ćsentés en Afrique tropicale, mais dans la rés̆on du Mozambique, telle que la comprend l'état-major de liew, le nombre des espèces était relativement restreint; on $y$ signale :

FIG. ‡. - Colonne rue de profil (\$/1).

Fig. 8. - Colonne avec capuchon anthérifère enlevé 8,1 ).

FıG. 9. - Masses polliniques vues par leurs points d'attache (20/1).

Fig. 10. - Coupe transversale de l'ovaire $(30,1)$. 
A. alcirome Reiehb. f. - Shire.

A. Kirlici kolfo ( = 1. bilobun var. Kirkii Rrichb.f.). - Environs de Mombasa. Kilimanjaro, Usimbara.

A. Kotschyamum Reirlsb. f. - Kilimanjaro, Pangani Valley, Shire, Mont Mlanje.

1. prchyurum Rolfo. - Ifripue orientale auglaise.

A. dives Rolfe. - Kilinanjaro.

A. Giryamae Rendle - Zanzibar, Magila, Kilimanjaro.

A:tridac'ylites Rolfe. - Vide supra.

A. fimbriatum Rendle - Usagara Mountains.

A. Smithii Rolfe. - Kilimanjaro.

A. chiloschistue Reichlb. f. - Usambara, Zambese, Shire.

A. megalorrhizum Rriehb. f. - Shire, Nyassalant.

Wh ces espèces aucune, sauf peut-être le $A$. chiloschistae, n'a été indiquée daus le voisinage de la r'égion d'où provient la plante que nous avons firit figurer et que pour plus de certitude nous avons soumise ì $\mathbf{M}$. le $\mathrm{I}^{\mathrm{r}}$ Rolfe.

M. Ie Dr Rolfe, dans' sa monographie des Angraecum afrieains, insérée dans la Flora of tropical Africa (loc. cit. 1) 133-150), classe les espèces en deux grands groupes, en se basant sur la présence ou l'absence des feuilles le long des tiges, cette absenee n'étant il est vrai, pas eomplète, mais due simplement an fait que les feuilles sont très rapidement eaduyues. Par ses tiges plus ou moins allongées, parfois grimpantes, s'aeernchant aux branches d'ar'bres, par ses longues racines, le labelle floral très profondément trilobé, la plante que nous figurons appartient à 1 m groupe eonstitué par les cinq espèces suivantes:

A. Scottelii Rendle. - Ruwenzori, Butagu.

A. armeniarum Lindl. - Sierra-Leone.

A. tridactylites Rendle.

A. Whitfieldii Rendl. - Sierra-Leone.

A. fimbriaum Rendle. - Nil (environs de Mombasa), Usagara.

La première de ces espèces se différencie facilement par ses fleur's en faseicules axillaires; che\% les quatre autres, les fleurs sont disposćes en racèmes. Comme son nom l'indique, l'A. fimbriatum Rendle possède des lobes du labelle fimbriés, ce qui u'est pas le cas eliez notre plante. Quant à l'A armeniacum son éperon est nettement elariforme.

L.es deux dernieres espèces voisines, et mème parfois eonfondues, se distinguent par la distance des fleurs sur le rachis; celles.ci, comme le montre la figure 1 de rotre planche, sont placées à au moins $4 \mathrm{~mm}$. de distance chez l' $A$. tridactylites, elles sont sitnces it 2,5 mm. maximum de ristanee eher l'A. Whitficldii. 

Pliovae Herh HORT THENENSIS.

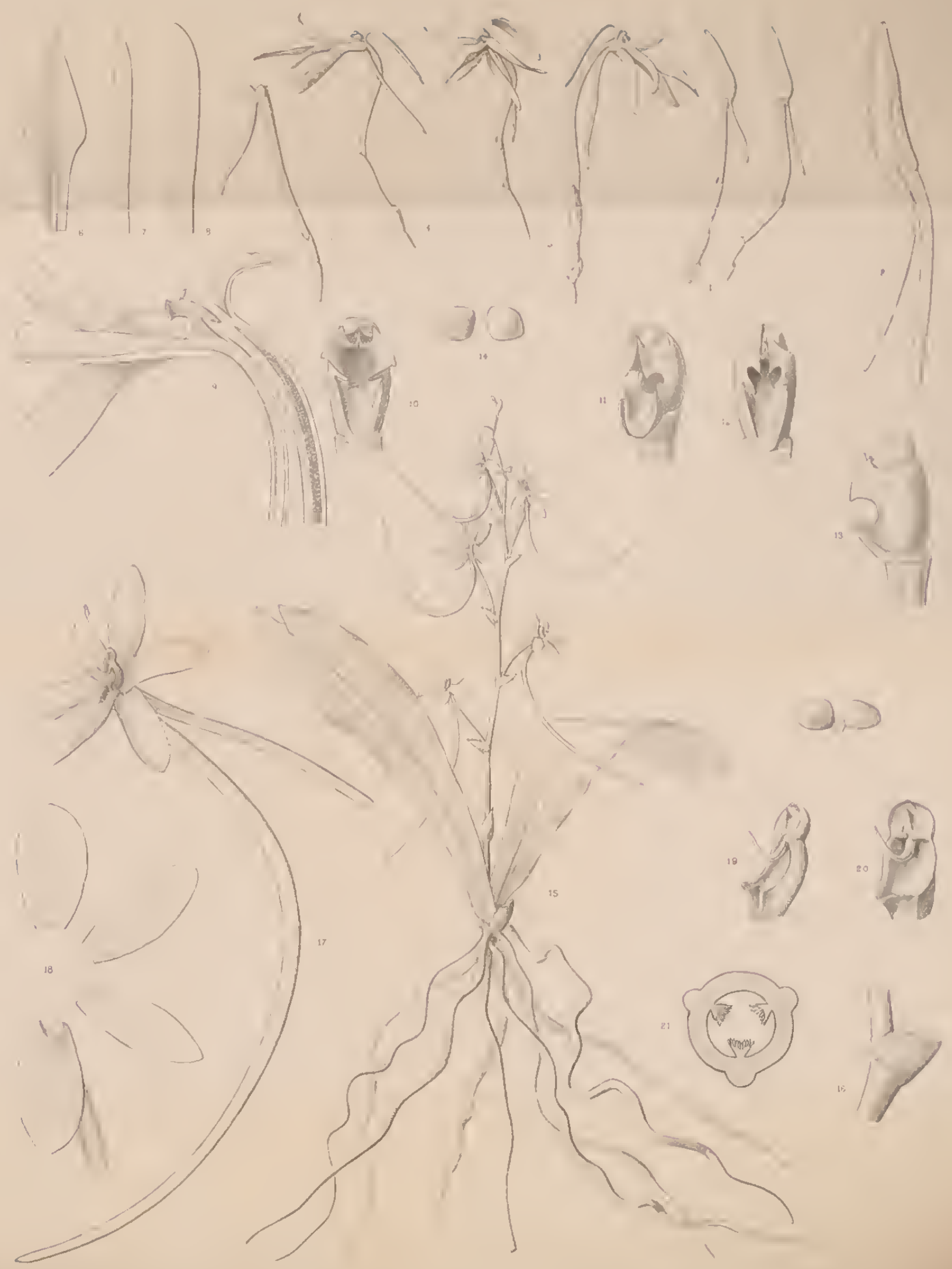

A. dAprevis, ad as del 


\section{Angraecum filicornoides the ITild. nov. sp.}

Angraecum flicornoides; labello lanceolato, circ. $2 \mathrm{~cm}$. longo, sublastato, acuto; sepalis et petalis lanceolatis subaequantibus, calcare gracile, filiforme, longissimo, cire. $2-3 \mathrm{~cm}$. longo, pedunculo basi bracteato, bracteis raginantibus; foliis ignotis.

Tiges et feuilles inconnues. Pédoncules axillaires, griles, plus ou moins flexueux, simples, uniflores, 'ganmis dans leur partie inférieure de gaines assez corlaces, aiteignant $1 \mathrm{~cm}$. de longr, subohtuses au sommet, carenées sur le dos. Fleurs blanches i sépales postérieur et latéraux, suhégaux, lancéolés ou légèrement obliqnement lancéolés, le supéricur dressé ou reéfléchi, les latéraux pendants, de $2 \mathrm{~cm}$. environ de long sur $3 \mathrm{~mm}$. de large. Pétales $u$ peu plus petits et moins linges que les sépales ćtalés. Labelle lancéolé, subhasté, aussi long que les pétales, plane, éperon pendant, fili-

\section{Explication des figures 1 à 14 de la planche XXI.}

Fia. 1. - Deux tiges floriferes en boutons (1/1).

Fic. 2. - Bouton (2/1).

Fig. 3. - Deux tiges florifères, fleurs épanouies (1/1).

Fig. 4. - Fleur épanouie (1/1).

Fig. 5. - Ovaire après fécondation, les pétales et sépales enlevés (1/1).

Fro. 6. - Labelle vu de face $(21)$.

Fır. T. - Pétale (2/1).

Fif. \&. - Sépale (2/1).

Fic, 9. - Coupe transversale de la base de la fleur $(4,1)$

Fit3. 10. - Colonne vue de face, les lobes latéraux écartés (8/1).

FIG. 11. - Colonne vue de trois quarts (8/1). 
forme, grèle de 2 i $3 \mathrm{~cm}$. de long. Colonne courte fortement concave, munie de chaque côté d'une aile latérale anguleuse dont les pointes se réunissent par derant.

\section{Hab. - Région de Mozambique : Morrumbala, 1900 (Éd. Luja).}

Observations. - Cette curieuse espèce, dont nous n'avons pas recu les feuilles, se rapproche singulièrement de l'Angraecum filicornu Thouars décrit en 1822 par le botaniste français dans son mémorable ouvrage sur les Orchidées des illes australes d'Afrique. L'espèce y a mène été figurée et une excellente planclie en a été donnée récemment dans le Dictionnaire des Orchidées (Angraccum, pl.9, déc. 1903). Comme on pourra en juger en comparant les figures de notre planche arec colle publiée par M. (roossens dans le " Dictionnaire iconographique des (Trehilées ", l'A. filicornu est dans toutes ses parties plus développé que la plante nouvelle. Pour domner une ilée de la proportion de certains éléments. nous dirons "fue le type de Thouars, originaire de Madagascar" et de l'ile Maurice, possìde un labolle et des pétales et sépales d'au moins $3 \mathrm{~cm}$. de long, un éperon atteignant de 10 à $13 \mathrm{~cm}$.

Une forme analogne ì l'Angraecum, que nous avons désignée filicornoides pour rappeler ses aflinités avec l'A. filicornu, ne semble pas aroir été rencontrée sur le continent Il est vraiment regrettable que nous nayons point reçu des feuilles de cette mème espèce, afin de pouvoir en donner une description plus complete.

Fig. 12. - Colonne rue de face, lobes latéraux rapprochés $(\& / 1)$.

Fici. 13. - Colonne vue de profil (\&/1).

Fio. 14. - Masses polliniques 20,1). 


\section{Mystacidium appendiculatum the Will. nos. sp.}

Mystacidium appendiculatum; ! lanta epiphytica ; folia oblonga apice inequaliter biloba, coriacea, erectu-patentia, $8 \mathrm{~cm}$. circ. longa et 17-18 mm. lata; 1acemus laxe 6-8 florus, sape flexuosus, blacteis cucullatis, vaginantibus, (irc. i mm. longis; perianthii segmenta subaequalia patentia, ovato-oblouga, subobtusa, $7 \mathrm{~mm}$. circ. longa, label!o obtuso basi non vel obcure et ubtuse denticulato, calcare elongitti, filiformi, subrecurvati, $4 \mathrm{~cm}$. circe longi; columma blevis, concaris, rostellum appendiculatuin; ovario tricostato.

Plante épiphryte, à tige trés courte ou mulle, à raciurs nombreuses, grèles, allongées. Fenilles an nombre de denx, inégalement liblobées au sommet il lobes subaigns, coriaces, dressées-étalées, de 8 cm. environ de loner et de 17 a $18 \mathrm{~mm}$. de large. Intlorescence en racène de 11 rm. environ de long, liche, a $6-8$ fleurs, munie diuns sal partie införieure d'une ou deux bractées phus ou moins engainantes, subaiguës. Bractées llorales engainautes, de J̀ 1111 . environ de long, obtuses. Pédicelle tloral grèle, atteignant $14 \mathrm{~mm}$. environ de long. Sépales elliptiques ou ohoviales, le postérieur un peu plus grand que les lintéranx, tous subobtus, de 6- $7 \mathrm{mmn}$. de long et 2 a $2,5 \mathrm{~mm}$. de large; pétales de mème tor'me et de mème grandeur environ que les sépales.

\section{Explication des figures 15 à 21 de la planche XXI.}

Fıa. 15. - Plante fleurie (1/1).

Fia. 16. - Bractée florale (6/1).

liı. 17. - Fleur épanouie (11).

Fı. 18. - Sépales, petale et labelle isolés (5/1). 
Labelle ovale, obtus, do $7 \mathrm{~mm}$. environ de longr, obscurément denticulé à la base, dent constituée simplement par l'angle brusque formé au niveau de la naissance de l'éperon ; celui-ci allongé, filiforme, recourbé, atteignant $4 \mathrm{~cm}$. de long. Colonne courte, concave, munie à l'extrémité du rostellum d'un appendice recourbé, allongé. Ovaire subcylindrique à 3 còtes correspondant aux placentas.

\section{Hab. - Région de Mozambique : Morrumbala, dans la forêt, décem- bre 1900 (Éd. Luja, n. 466;.}

Observations. - Nous avons été amenés à rapporter cette plante plutòt au genre Mystucidium qu'au genre Listrostachys, par l'aspect geénéral, car nous n'en avons pu voir de pollinies bien développées.

Aucune des espèces décritcs par $\mathrm{M}$. Kolfe dans la .. Flora of tropical Africa (VII, 1. 169 et suivantes) ne parait avoir de grandes affinités avec notre plante; seules certaines formes du sud de l'Afrique, telles par cxcmple les Mystacidium filicornu Lindl. et Mystacidium gracile Harvey, semblent avoir certaines affinités avec notre plante quant au port et aux feuilles, mais chez ces deux espèces les fleurs sont à lobes du pér.anthe très' nettement aigus, tandis qn'ils sont nettement subobtus dans notre plante, comme le font d'ailleur's très bien ressortir les tigures 17 et 18; en outre, les delux plantes que nous venons de citer préscntent une dent trìs nette à la base du labelle; chez le M. appendiculatum la dent bas.laire est très obscure, quasi nulle. La forme de la colonne est également très différente et l'appendice qui prolonge le rostelium sur la face antérieure donne un caractère très particulier à la fleur ; c'est de ce caractère que nous avons tiré le nom spécifique. On pourra d'ailleurs juger des différences en comparant notre planche à celle des "Orchids of South Afriea " (pl. 55 et 56 ), le beau et savant travail de M. Harry Bolus qui est toujours à consulter par cenx qui ont à s'occuper des Orchiclées de la partie méridionale de l'Afrique centrale.

Fio. 19. - Colonne vue de profil avec l'appendice du rostellum (11/1).

Fui. 20. - Colonne rue de face (11/1).

Fra. 21. - Coupe transversale de lovaire $(9,1)$. 


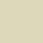





\section{PLANT $\nRightarrow$ NOV E}

VEL MINUS COGNITE

FX HERBARIO HORTI TIENENSIS

PLANTES NOUVELLES OU PEU CONNUES

CONTENUES DANS L'HERBIER

$\mathrm{DE}$

L'HOETUS THENENSIS

AVEC LES DESCRIPTIONS OU ANNOTATIONS

DE

M. Ém. DE WILDEMAN

DOCTEUR EN SCIENCES

CONSERVATEUR AU JaRDin BOTANIQUE DE l'État

Quatrieme livnatson.

(SEPTEMBRE 1905)

VWUVE MONNOM

IMPRIMHUR F:T HID I'WUK

32, RUE DE LINDUSTRIE, 32

BRUXELLES

Se vend exclusivement à l'Administration des biens et affaires de M. van den Bossche, 5, Grande Montagne, à Tirlemont. 



\section{QUATRIEME LIVRAISON}

J'ai choisi les matériaux d'études levant servir à la quatrième livraison de nos Planta nova vel minus cognita dans la belle collection des plantes de la West Australia recueillie par M. le $\mathrm{D}^{\mathrm{r}} \mathrm{E}$. Pritzel, une série de doubles de cette collection m'ayant été cédée par le Muséum Royal de Botanique de Berlin.

En cela faisant, j’ai eu à cơur d'apporter, dans la mesure des éléments dont nous pouvious disposer, quelque contribution nouvelle à la flore austrulienne, si riche et $\mathrm{s}$. intéressante, de témoigner de la sorte ma gratitude ì MM. Alex. Morrison et J. II. Maiden, les savants botanistes du gouvernement dans l'Australic occidentale et dans la Nouvelle-Galle du Sud : grâce à leur bienveillance, aux précieux envois de graines qu'ils veulent bien me faire, l'un et l'autre, les collectious de l'Hortus Thenensis s'enrichissent tous les aus d'espèces rares et curieuses.

J'ai proposé it M. le $\mathrm{D}^{\mathrm{r}}$ De Wildeman de prendre pour sujei d'étude la famille des Protéacées, représentée dans cette flore australienne par environ 600 espèces et remarquable a tant d'égards par la variété de ses types, comme aussi par les exemples de polymorphisme qui se rencontrent dans un assez grand nombre de cas. Et comme les dimensions de notre publication ne permettent pas d'embrasser, dans une seule livraison, un sujet aussi vaste, j’ai cru devoir le circonscrire à la première des deux sous-familles, celle des Persoonioideo.

J'aurais voulu présenter au moins un type de clıcun 
des genres australiens de cette sous-famille. Mais les matériaux dont nous disposons, ne nous ont pas permis de réaliser entièrement ce programme : pour tel genre dont l'habitat se trouve dans d'autres régions du continent australien, les éléments nous manquaient; pour d'antres, pour le Franklandia, par exemple, ces éléments étaient insuffisants pour fournir des analyses intéressantes.

M. De Wildeman a donc dû se restreindre et n'a pu envisager que $\delta$ genres distincts, en choisissant dans chacun d'eux, et autant que faire se pouvait, des espèces appartenant aux différentes sections des dits genres qui n'avaient pas encore été figurées dans d'autres recueils. Il a pensé en outre qu'il convenait de faire précéder son travail de quelques considérations générales sur la sousfamille des Persoonioidece et sur les sections qui s'y trouvent incluses, comme aussi sur les genres étudiés. C'est pourquoi cette quatrième livraison des Planta. Norce ne se présente pas tout à fait dans le mème cadre que les précédentes.

Après le beau travail de MM. L. Diels et E. Pritzel, intitulé fragmenta Phytographia Australice occidentalis, qui a paru, l'an dernier, dans le trente-cinquième volume des Botanische Jahrbiicher, j'arais à me deminder si le moment était bien choisi pour reprendre un tel sujet. Mais en maintenant cette étude dans le domaine strictement limité de la systématique, nous hui arons assigné un but quelque peu différent de celui des sarants allemands. Nous pouvons en effet donner dans cette livraison des analyses de vingt-trois espèces qui n'avaient pas été figurées jusqu'à ce jour et nous pensons, au surplus, qu'en botanique, il n'y a pas de besogne, si modeste qu'elle paraisse, qui ne soit de quelyue profit pour' l'a vancement de la science.

L. B. 
I)ans la fanille des Proleacea, les Persoonicidece se différencient des Grevilleoidece par leur's tleurs solitaires à l'aisselle d'une bractée et par leurs ovaires, souvent uniovulés, qui se transforment en un fruit sec, noix ou drupe.

Cette sous-famille a été à son tour divisée en quatre groupements ou sections, dont les caractères différentiels ont été établis par M. le professeur Engler dans les Natürliche Pfanzenfamilien, et qui peuvent se résumer dans la clef analytique qui suit:

\section{Périanthe régulier à lobes libres. Axe floral non cupulé . . . . . . . . Persooniea. \\ Périanthe régulier' à tube étroit, cylindrique non fendu. Axe floral cupulé à la base . . Franklandiea. \\ Périanthe régulier ou zygomorphe, se fendant. Axe floral non cupulé . . . . . . . Proteea. \\ Périanthe zygomorphe. Axe floral non cupulé Conospermea.}

La tribu des Persooniece comprend neuf genres dont un seul sera étudié dans les notes qui vont suivre. les autres types se trouvant localisés dans la Nouvelle-Galle du Sud et la Tasmanie (Bellendena B. Br., Symphyonema B. Br., Agastachys B. Br. et Cenarrhenes Labill.), voire mème sur d'autres points du globe. Ces neuf genres se classent en trois gronpes en se basant sul la présence ou l'absence d'écailles ou d'un disque à la base de l'ovaire.

Les Persoonia, comme le montrent plusieurs figures de la planche XXII, ont 4 écailles à la base de l'ovaire et partagent ce caractère avec les Gamieria Brongn. et Gris, Dilobeia Thou., Beauprea Brongn. et Gris et Cenarrhenes. Le premier de ces genres possède plusieurs ovules dans l'ovaire et plusieurs graines dans le fruit, et cette donnée caractéristique le sépare de tous autres. Chez les Dilobeia et Beauprea, les fleurs sont en épis, réunies souvent en panicules, tandis que chez les Persoonia et Cenarrhenes elles sont en épis axillaires ou en fascicules, ou même solitaires-axillaires. Le monotypique Cenarrhenes nitida Labill. (de la Tasmanie) se différencie des Persoonia par ses ovaires toujours à une ovule pendante, et ses fleurs toujours disposées en épis axillaires; mais ce dernier caractère, qui a bien sa valeur, n'est pas toujours nettement établi, et, en effet, les Persoonia, par' suite 
d'avortement, présentent parfois des inflorescences similaires à celles du Cenarrhenes, bien qu'en général les fleurs soient solitaires à l'aisselle des feuilles, ou que l'avortement des feuilles fasse paraitre les inflorescences en épis comme si elles étaient terminales.

Nous n'avons aucune remarque spéciale à présenter sur la tribu des Franklandien, qui comprend le seul genre Franklandia R. Br. et dont nous ne pouvons malheureusement founir aucune donnée analytique.

Le groupement des Protee comprend quatorze genres. Parmi eux, les Simsia R. Br. occupent une place très spéciale par leur's étamines dont les anthères sont soudées en une sorte de cylindre entourant le style, ainsi que le montreront nettement les figures analytiques de la planche XXVIII. Dans tous les autı'es genres les anthères sont libres; les uns, Faurea, Protea, Leucospermum, Leucadendron et Aulax. tous africains, ont des fleur's nettement zygomorphes et, après l'anthèse, on voit le lobe postérieur se détacher nettement des autres lobes du perianthe; quant aux autres, - parmi lesquels les Isopogon, Petrophila et Adenanthos sont seuls australiens, tandis que les Sorocephalus, Nivenia, Serruria, Mimetes et Spatalla habitent l'Afrique australe ils ont au contraire des fleur's régulières ou parfois très légèrement zygomorphes. Et dans ce second groupement, on peut établir une sousdivision en se basant sur la présence ou l'absence d'écailles à la base de l'ovaire : l'absence d'écailles est caractéristique pour les Isopogon et les Petrophila, la caducité des bractées différenciant les premiers des seconds qui ont au contraire ces mêmes organes persistants; parmi les genres quí possèdent des écailles, les Adenanthos, seuls australiens, se caractérisent par la chute du périanthe, qui laisse à la base une sorte de cupule.

Enfin la tribu des Conospermea ne comprend que les deux genres Synaphaca et Conospermum. Les caractères différentiels résident dans l'avortement de l'une des anthères, qui se produit différemment, et dans la forme des feuilles qui sont divisées chez les Synaphoa et entières, tout au moins en ce qui concerne les feuilles supérieures, dans les Conospermum. 


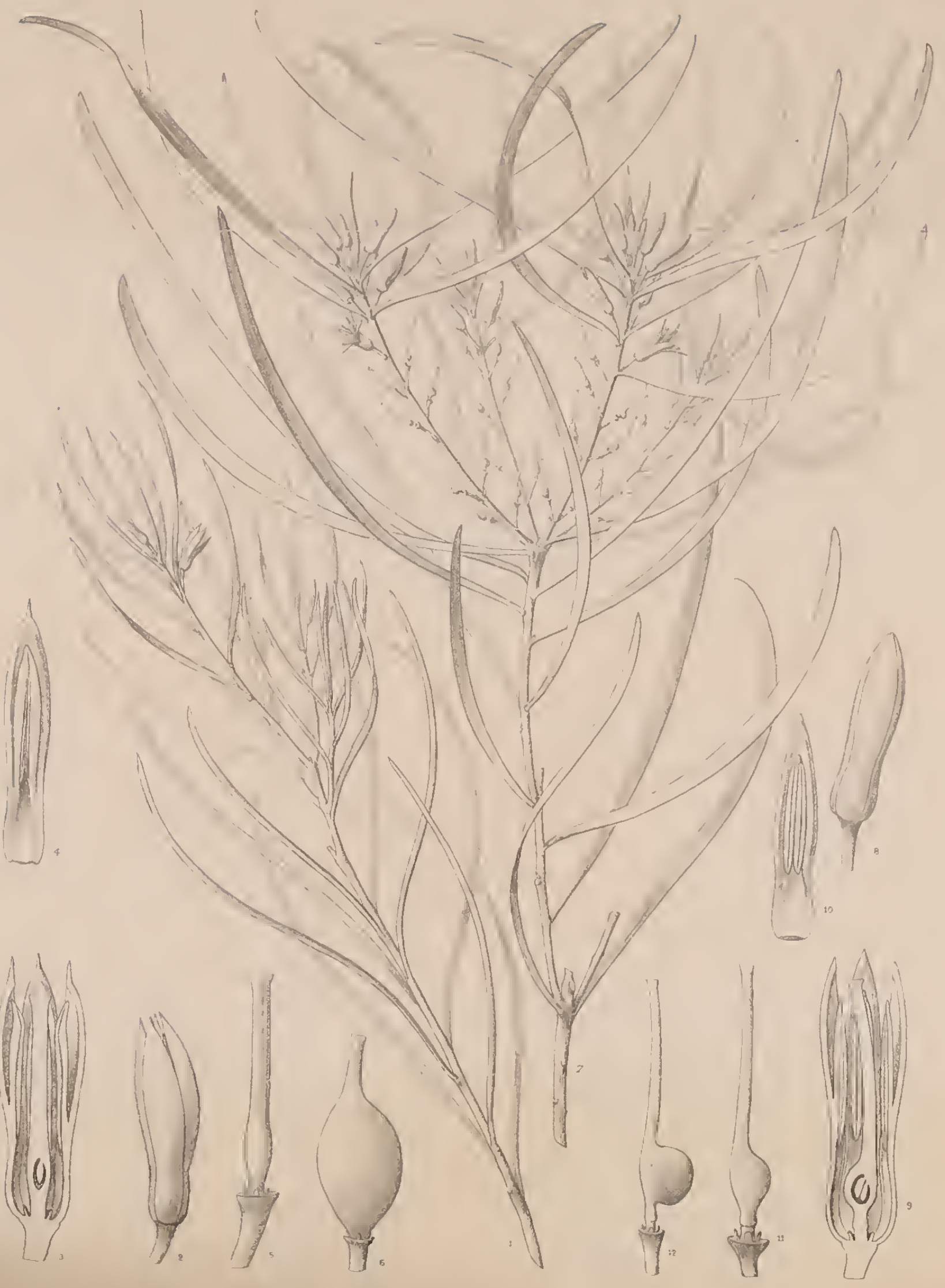




\section{PERSOONIA S SM.}

Le genre Persoonia, créé par Simith dans les transactions de la w linnean Society "de Londres en 1798, a été dédié au botaniste Persoon dont les travaux très importants de systématique végétale, méritaient cettc dédicace. Il contient environ une soixantaine d'espèces qui toutes, sauf une, appartiennent à la flore de l'Australie continentale.

Ce genre est caractérisé par ses feuilles entières, ses fleur's blanches ou jaunâtres, solitaires à l'aisselle des feuilles ou constituant parfois par l'avortement de ces dernières un court racème; parfois même le racème devient unilatéral. Ces fleurs sont hermaphrodites; leur périanthe est régulier, à 4 lobes libres ou presque libres jusqu'à la base. Les étamines sont toutes fertiles, chacune d'entre elles opposée au lobe du périanthe. L'ovaire, plus ou moins nettement stipité, est entouré à la base de 4 glandes hypogynes et terminé par un style droit, parfois réfléchi; les ovules sont, géminés et pendants dans la cavité ovarieme, étant attachés sur le côté vers le haut de la cavité. A la fleur succède un fruit drupacé, à exocarpe charnu, à cndocarpe très dur, prarfois uniloculaire à une graine, d'autre fois biloculaire à deux graines.

Bentham, dans la Flora Australiensis, a réparti les différentes espèces de Persoonia en trois sections qui sont encore admises actuellement. Les caractères de ces sections sont :

Section I. - Pycnostyles : Style court, souvent aussi épais que l'ovaire, recourlué vers le sommet, portant le stigmate dans une excavation du segment supérieur du périanthe, en dessous de l'anthère.

Section II. - Acranthera : Style allongé déprassant les anthères, i stigmate terminal. Conncctif des anthères prolongé au delà des loges.

Section III. - Amblyantherce: Stylc allongé dépassant les anthères, à stigmatc terminal. Connectif des anthères non prolongé au delà des loges. 
$\mathrm{Au}$ sujet de ces trois sections, MM. Diels et Pritzel font remarquer dans leurs Fragmenta Phytographice Australice occidentalis, p. 145, que les deux dernières sont très voisines.

Parmi les plantes distribuées par M. le $\mathrm{D}^{\mathrm{r}}$ Pritzel dans ses Planta Australice occidentalis, nous avons choisi deux types qui n'avaient pas encore été figurés et nous les avons soumis à l'analyse: ces deux types $P$. diadema F. v. Muell. et $P$. longifolia $\mathrm{R}$. Br. appartiennent d'après les auteurs, le premier, à la section Pycnostyles, le second, à la section Amblyanthera.

Avant de passer à l'examen de ces espèces, nous tenons à faire ressor'tir que nos analyses ne nous permettent pas de décider si vraimentles données proposées par Bentham sont caractéristiques. Le caractère du style de notre $P$. diadema ne correspond pas tout à fait à celui de la descripption du baron von Mueller ni à celui de la section. Peut-ètre cette différence est-elle due au mauvais état des matériaux que nous avons regus et qui, pour les autres caractères, concordent fort bien avec les descriptions.

\section{Persoonia diadema F. v. Mucll.}

Fragm. phyt. Australixe X (1876) p. 46.

Plante frutescente, à rameaux légèrement séricés, glahrescents. Feuilles longuement linéaires épaisses, plus ou noins trigonées, sillonnées, pointues au sommet. Fleurs solitaires à l'aisselle des feuilles supérieures, formant parfois dans l'ensemble une sorte de corymbe. Périanthe à 4 lobes lancéolés, assez épais, éparsement relus sur le los,

Explication des figures 1-6 de la planche XXII.

Persoonia diadema $r$. Muell.

Fii, 1. - Rameau florifère (1/1).

Fin. 2. - Fleur (4/1).

Fti 3. - Coupe longitudinale de lin fleur (5/1).

Fis; 4 - Un des lobes du périanthe vu le face (5/1).

Fig. 5. - Ovaire, style et stigmate (5/1).

Fig. 6 - Ovaire après fecondation (4/1). 
terminé par un acumen aigu. Étamines subohtuses soudées sur la moitic de leur longueur avec les lobes du périanthe, atteignant presque le sommet des lobes. Ovaire entouré à la base par 4 glandes lıypogynes peu développées, se terminant par un style droit brusquement tronqué au sommet, aussi long que les étamines, un peu plus court que le périanthe. Fruit ovoïde terminé par une sorte de col constitué par le style persistant.

\footnotetext{
Hab. - District de Coolgardie : Mont Ghurchman (Young): région du lac Deborah près de Ghoodi et près de Karalee (E. Pritzel).
}

Observations. - Le $P$. diadema avait été, comme nous venons de le signaler, récolté pour la première fois dans le district de Coolgardie sur le mont Churchman par Young. M. le $\mathrm{D}^{\mathrm{r}}$ Pritzel a su l'occasion de l'observer pendant son voyage vers le sud et l'a récolté en lleurs, près de Ghooli, en octobre 1901. C'est une plante de cette provenance qui a servi i dessiner les figures 1 à 6 de la planche XXII; M. 1)iels a recueilli la nème espèce près de liaralee en novembre de la mème anuée. Le baron F. v. Mueller en décrivant cette espèce la signale comme voisine du $P$. Saundersiana liepp. également originaire de l'Australie occidentale. Parmi les caractères différentiels il cite les feuilles; en efret tandis que chez la dernière espèce citée elles sont planes, chez le $P$. diadema elles sont plus ou moins trigones et présentent en tous cas deux sillons bien malqués sur la face supérieure. Il cite encore une diflérence dans le nombre des glandes hypogynes qui dans le $P$. diadema seraient an norobre de 3 , la glande postérieure étant avortée.

Nous avons déjà insisté plus haut, à propos des caractères des sections établies dans le gemre, sur ce fait que le style de la plante que nons figurons ne paraissait pas présenter le stigmate latéral caractéristique des Pycnostyles, et nous regrettons de ne ponvoir nous arrêter davantage sur ce point, les matériaux dont nous disposions étant très précaires. Mais nous insistons sur l'acumen des lobes du périanthe qui parait constituer une bonne différenciation. Quant aux étamines, contrairement à ce que dit la description de l'auteur elles sont plutôt aiguës qu'obtuses. Nous pourrions encore faire observer que dans la plante que nous arons sous les yeux le périanthe n'est pas tout à fait glabre, mais muni, comme le fait voir les figures 2 et 3 de la planche XXlI, de quelques poils argentés qui, à l'état sec, tranchent très nettement sur la couleur noire du limbe.

Ces petites divergences, peut être accidentelles ou dues à l'àge de la plante étudiée, nous ont amené à faire figurer ces détails dont la discussion mériterait d'ètr' faite à l'aide de plus nombreux matériaux. 


\section{Persoonia longifolia $R . B r$.}

in Trans. Linn. Soc. X (1810) p. 164 et Prod. p. 374; Meisn. in Pl. Preiss. I p 533 et in DC. Prod. regn. veget. XIV p. $343 ;$ Benth. Fl. Austral. V p. 390.

Persoonia Drummondii Lindl. Swan River App. (1810) p. 35.

Petit arbrisseau ou petit arbre atteignant 6 mètres de hauteur, glabre ou légèrement pubescent argenté à l'état jeune. Feuilles linéaires-lancéolées, de 12 à $20 \mathrm{~cm}$. de long, subobtuses ou terminées par une sorte de mucron, rétrécies ver's la base en un pétiole court, à nervures médiane et latérales plus ou moins parallèles; peu apparentes. Fleurs solitaires à l'aisselle de feuilles, mais formant parfois, par suite de l'avortement ou de la chute prématurée des feuilles, des racèmes terminaux ou subterminaux. Pédicelle assez grêle, de 6 à $12 \mathrm{~mm}$. de long. Périanthe légèrement duveteux-ferrugineux ou muni de quelques poils soveux, atteignant $10-12 \mathrm{~mm}$. de long; lobes du périanthe allongés, aigus au sommet. Étamine soudée ¿i la base avec le lobe correspondant du périanthe, ‘̀ loges parallèles, aiguës au sommet. Ovaine ovoïle, aplati, glabre, stipité, stipe articulé sous l'ovaire et muni ì sa base de 4 glandes hypogyres espacées, ovules pendants ; style allongé, légèrement renflé au sommet, environ aussi long que le ! érianthe. Fruit drupacé, plus ou moins oblique, de $10 \mathrm{~mm}$. environ de diamètre.

Explication des figures 7-12 de la planche XXII.

Persoonia longifolia $I i r$ ir.

FIG. ¿. - Rameau fleuri (1/1).

Fig. S. - Bouton floral (4/1).

FIG. 9. - Coupe longitudinale de la fleur (5/1).

Fif. 10. - Lobe du périanthe vu de face (4/1).

FIg. 11-12. - Ovaires, styles et stigmates à divers états de déreloppement (5/1). 
Hab. - District South West Plantagenet (Pritzel); King George's Sound jusquà la Swan River (R. Brown et autres); Vasse River (Mrs Molloy).

Observations. - Cette espèce qui parait aimer les endroits asser, liumides, appartient à la secticn Amblyanthera que nous avons caractérisée plus haut en exposant sommairement les caractères du genre. Par ses étamines à connectif non prolongé au delà des loges de lanthère et surtout par un caractère secondaire, celui de l'articulation de l'ovaire, il ne peut y avoir le moindre doute sur le rapprochement à établir. Ce dernier' caractère est d'ailleur's celui sur lequel s'est basé Bentham pour distribuer en deux sous-sections les 37 espèces qui se rangent dans les Amblyanthera et, chose rentarquable, les trois espèces il ovaire nettement articulé au-dessus de la base, comme c'est le cas cliez notre plante (fig. 9, 11 et $1: 2$ ) et qui ont des affinités manifestes, appartiennent it la flore de l'Australie occidentale; toutes les autres à ovaire non articulé ou articulé tout à fait ì la base, sont de l’Australie orientale.

Les trois espèces entre lesquelles il aurait fallu choisir pour déterminer la plante figurée, sont, outre le $P$. longifolia, les $P$. urticulata $\mathrm{R}$. Br. et $P$. elliptica $\mathrm{R}$. Br. Cette dernière, caractérisée par ses feuilles largement ovales ou elliptiques atteignant $7,5 \mathrm{~cm}$. environ de long, ne pert être confondue avec le $I^{\prime}$. longifolia qui, comme le montre la figure $\tau$ de la planclie XXII, possède des feuilles nettement linéaires ou linéaireslancéolées. Quant au $P$. articulata, il est pour la forme des feuilles plus ou moins intermédiaire entre les deux autres espèces : ces feuilles sont oblongues lancéolées, pouvant atteindre jusque $15 \mathrm{~cm}$. de long alor's que celles du $P$. longifolia atteignent $20 \mathrm{~cm}$.; mais tandis que les feuilles de cette dernière espèce sont comme le montre très bien notre planche, nettement falciformes, elles sont droites, rigides chez le $P$. articulate. 

(19)

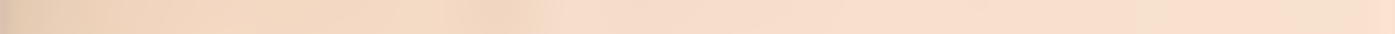




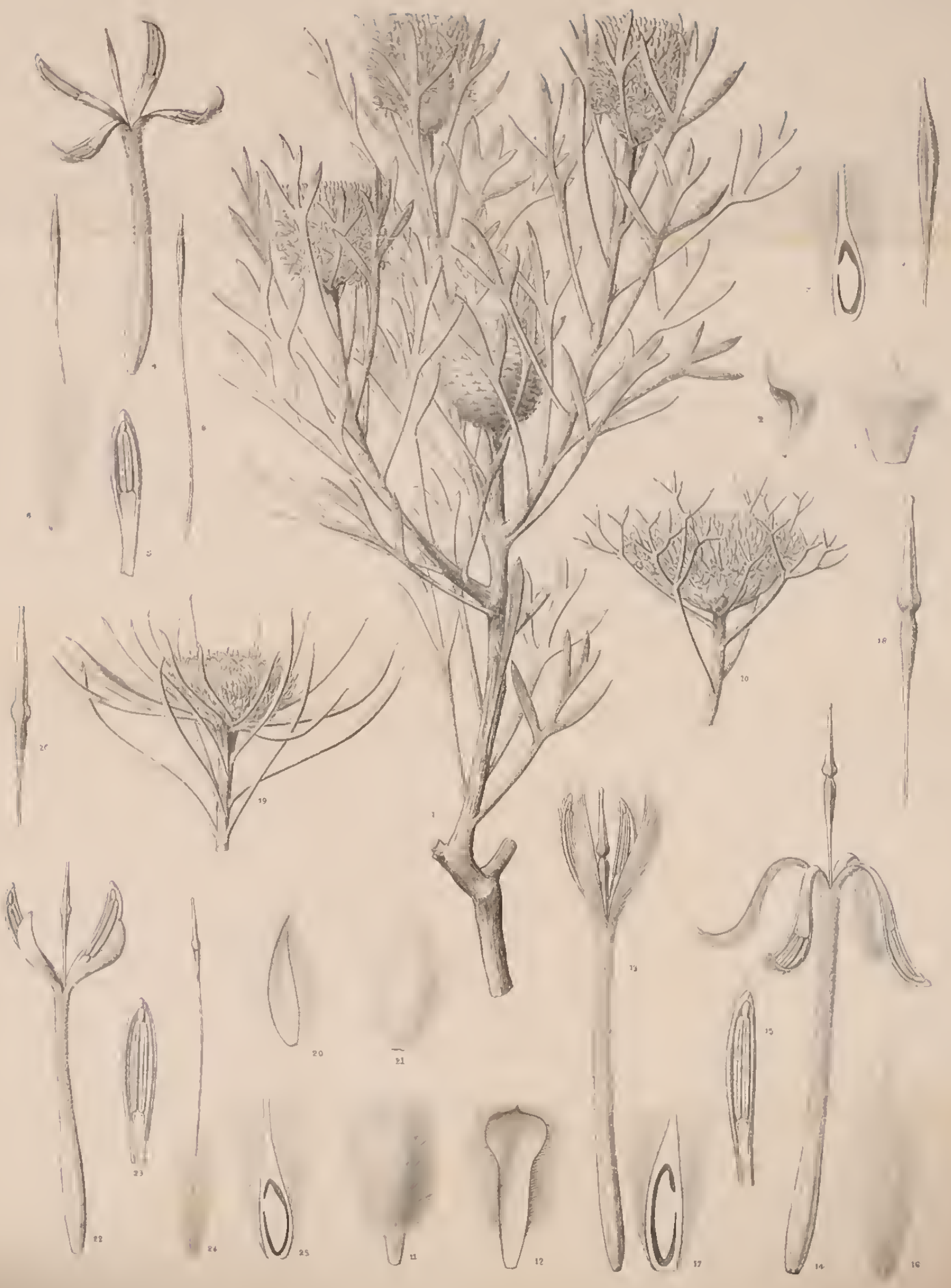

AdApreval ad nat del 

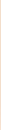


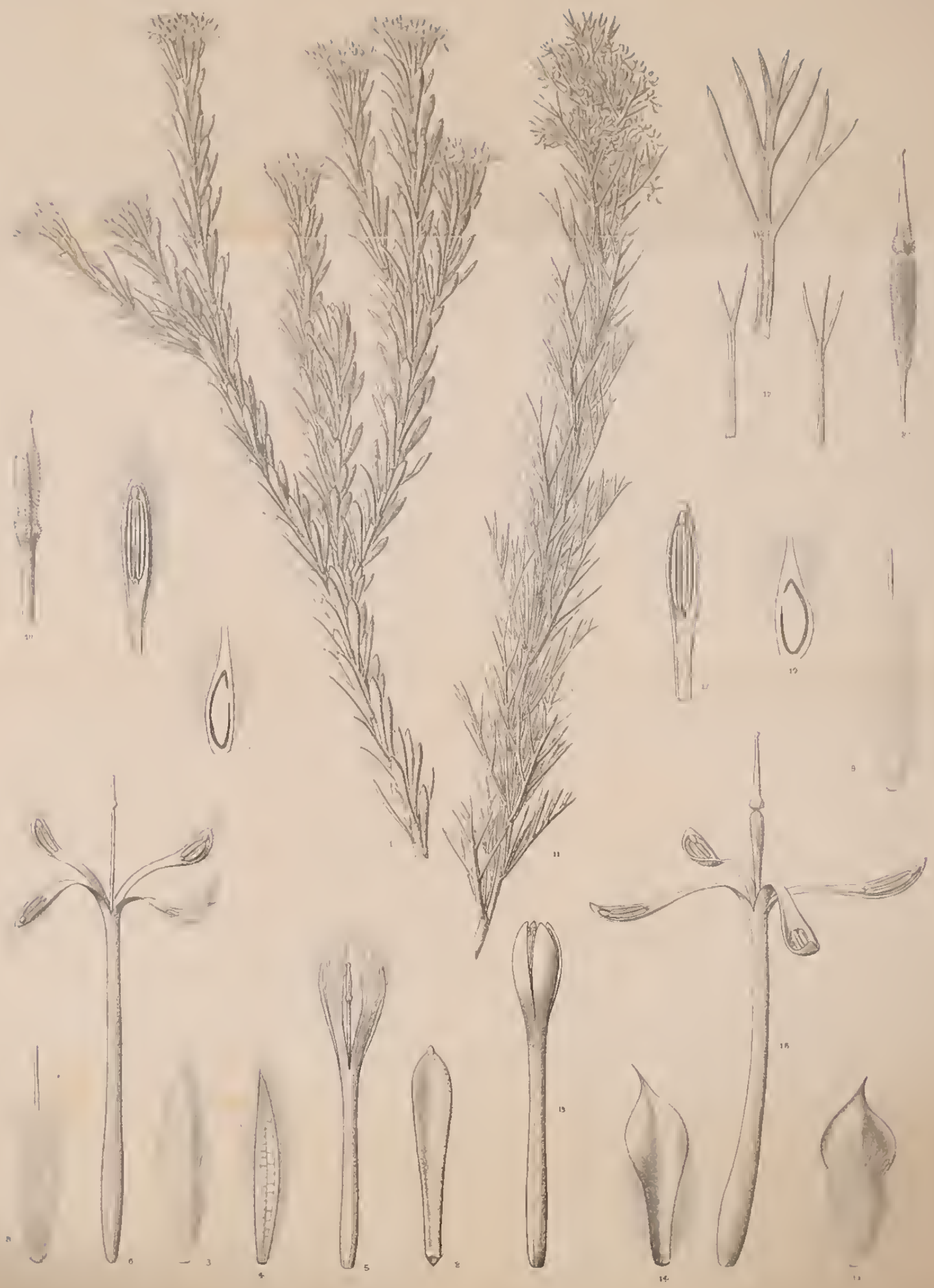




\section{ISOPOGON R. Br.}

Le genre Isopogon, créé par R. Brown, est très voisin du genrc Pelrophila. Les espèces assez nombreuses qui le composent, une bonne trentainc, sont toutes localisées dans l'Australie extratropicale et en particulier dans la West-Australia; il en existe quelques espèces dans lc South Australia, le New South Wales et le Queensland.

Les Isopogon sont caractérisés par des fleurs hermaphrodites à périanthe régulier dont les 4 lobes sont linéaires ou oblongs, variant du l'ose au lilas, disposćes en cônes plus ou moins denses, munis à leur base de bractées imbriquées formant un involucre parfois caché par les feuilles. C'est sur le fruit qu'est basée la différenciation la plus nette des genres Isopogon et Petrophila : dans ce dernier les écailles tombent tandis qu'elles persistent chez les Isopogon, comme nous l'avons dit en examinant plus haut les affinités et différences des diver's genres dont nous avons pu faire figurer des représentants.

Les Isopogon préfèrent les forêts peu compactes; on les rencontre aussi dans les bronsses sablonneuses où certaines espèces telles l' $I$. roseus Lindl. communiquent au pays un aspect très caractéristique. Bentham, dans la Flora Australiensis que nous suivons ici, classe les diverses espèces qu'il connaissait à l'époque de la publication de son magistral ouvrage, en deux sections dont nous résumerons ci-dessous la diagnose :

Section I. - Hypsanthus : Écailles florales acuminées, non fortement imbriquées, les internes étroites. Réceptacle plein, convexe ou rarement oblong. Feuilles généralement planes.

Section II. - Eustrobilus : Écailles largement dilatées ou tronqućes à l'extrémité, fortement imbriquées après la floraison. Réceptacle convexe, conique ou cylindrique.

Ces caractères, à première vue très tranchés, le sont beaucoup moins quand on examine les échantillons. C'est ce qui nous a amené à faire figurer en particulier les cinq espèces suivantes au sujet desquelles nous aurons à présenter certaines remarques. 


\section{Isopogon buxifolius R. $\mathrm{Br}$.}

in Trans. Linn. Soc. X (1810) p. 74 et Prodr. p. 367 ; Meisn. in DC. Prodr. regn. veget. XIV p. 282 ; Bienth. Fl. Austral. V p. 341.

Isopogon spathulatus R. Br. Prot.' Nov. (1S10) p. 8 ; Meisn. in Pl. Preiss. I p. 509 et Il p. 217 et in $D C$. Prod, regn. veget. XIV p. 282.

Plante buissonnante, foliacée, atteignant un peu plus de I mètre de haut, à rameaux et à jeune fenillage pubescent. Feuilles très variables dans leur forme, petites, rapprochées, atteignant rarement plus de 2 centim. de long, plus ou moins aplaties et concaves. Inflorescences terminales, cônes sessiles. Bractées externes du cône peu nombreuses, ciliées; les bractées florales lancéolées-linéaires, glabres extérieurement, densément velues sur le dos. Périanthe d'environ 12 millim. de long, divisé assez profondément en 4 lobes; tube du périanthe glabre, lobes velus sur le dos, surtout vers le sommet où les poils très longs forment pinceau. Étamines insérées dans l'élargissement du lobe, à connectif terminé au delà des loges en globule glabre. Ovaire densément velu, longuement ovale, à style grêle glabre, à stigmate plus ou moins fusiforme,

Explication des figures 1-10 de la planche XXIII.

Isopogon buxifolius $k$. $B r$.

FIG. 1. -- Rameau florifere (1/1).

FIG. :. - Feuille vue par la face intérieure (3/1).

F1G. 3. - Braclée florale vue par la face dorsale (6/1).

FIG. 4. - Bractée florale vue par la face interne (6/1).

Fig. 5. - Fleur avant son épanouissement complet (6/1).

FIG. 6. - Fleur épanouie (6/1).

Fır. 7. - Extrémité d'un des lobes dı périanthe avec une étamine vue de face $(10 / 1)$.

FIG. 8. - Ovaire et base du style (6/1).

FiG. 9. - Coupe longitudinale de l'ovaire (12/1).

FıG. 10. - Extrémité du style avec stigmate (17/1). 
muni de 1 lignes longitudinales de poils courts et réfléchis, se terminant avant d'atteindre le sommet.

\section{Hab. - District North-West Plantagenet (Pritzel); King George's Sound et régions adjacentes (R. Brown et autres).}

Observations. - I'I. buxifolius R. Br. appartient, eomme il est aisé de s'en rendre compte en examinant les figmes 1 à 10 de la planche XXIII, à la première des deux sections du genre que nous avons earactérisées plus hant. En effet, les éeailles (fig. 3 et 4) sont nettement acuminces et les feuilles sont planes. Nans cette seetion il existe treize espèces, toutes appartenant à la flore de l'Australie oceidentale. De ees espèees neuf possèdent des feuilles entières; les quatre autres: s.tridens F. v. Muell., S. Baxteri R. Br., S. roseus Lindl., S. adenanthoides Meissn. possèdent des feuilles dentées, tri- ou plurilobées.

Par la longueur de ses fenilles atteignant an maximum $5 \mathrm{~cm}$. de long et eela ver's la base de la plante seulement. l'I. buxifolius forme avee l'I. axillaris R. Br. un groupement secondaire en opposition aux sept autres espèces du sous-gemre dont les fenilles sont beaueoup plus développées et mesurent, sur les rameaux, de 5 à $15 \mathrm{~cm}$. de long.

Le nom seul de l'I. axillaris, nous indique déjà le caraetère différentiel : les infloreseenees sont axillaires le long des rameaux, ce qui permet de le différencier de l'I. buxifolius, dont les infloreseenees sont, comme le fait bien voir la figure 1 ( $p l$. XXIII), nettement terminales.

Bentham, dans sa Flore, insiste sur la variabilité des feuilles ehe\% eette plante et propose de subdiviser le tỵpe en quatre variétés qu'il dénomme eornme suit et dont les earactères sont :

Var. spathulatus. - Fenilles obovales-spatulées, leétrécies à la base en un pétiole, généralement de $12 \mathrm{~mm}$ environ de long.

Var. obovatus. - Fruilles obovales ou oblongues, légèrement rétrécies à la base, mais non pétiolées, de 12 à $24 \mathrm{~mm}$. de long.

Var. typicus. - Feuilles largement sessiles, ovales, à pointe courte reeourbée, de 6 à $8 \mathrm{~mm}$. le long.

Var. linearis $\mathrm{R}$. Br. - Feuilles étroitement oblongues ou linéaires, sessiles, de 12 à $18 \mathrm{~mm}$. de long.

Si l'on compare les figures de notre planehe avee les earactères que nous venons de donner, on verra que e'est des deux premières variétés que notre plante se rapproche le plus; pour la longueur des feuilles elle parait intermédiaire entre les deux; quant à la forme même spatulée ou obovale oblongue, e'est une question de plus ou de moins sur laquelle nous ne eroyons pas devoir insister. 
Los écliantillons figurés proviennent des forêts sablonneuses, à Eucalyptus marginatus, du district Murray, où ils ont été recueillis par M. E. Pritzel (Pl. Austral. occid., n. :269).

\section{Isopognon asper $R . B r$.}

Prot. nov. (1830) p. \&; Meisn. in Pl. Preiss. I p. 508 et in DC. Prod. regn, veget. XIV p. 278; Benth. Fl. Austral. p. 348.

Isopognn scaber Lindl. Swan River Apl) (1840) p. 34 (not of Bot. Mag.)

Arbrisseau à branches réduites simples, dressées, de 30 à $60 \mathrm{~cm}$. (le haut parfois beaucoup plus développé atteignant plusicurs pieds de hauteur, à rameaux pubescents à feuillage légèrement scahre. Feuilles atteignant au maximum 2,5 cm, environ de long̨, densément réunies le long des tiges, pennées, à segments fourchus ou trilobés, parfois réduites à un limbe fourchu ou trilobé; lobes des feuilles rigides, linéaires, aplatis, canaliculés sur la face supérieure. aigus. Inflorescences subglobuleuses, axillaires, disposées vers le sommet des ramifications, formant un bouquet feuillu terminal. Écailles du cône obovales-spatulées, toutes densément velues sur la face extérieure, glabres sur la face interne, aiguës au sommet, les intérieures plus étroites que les moyennes, toutes fortement imbriquées après la

Explication des figures 11-20 de la planche XXIII.

Isopogon asper $R . I B r$.

Fig. 11. - Rameau fleuri (1/1).

Fli. 12. - Feuilles differemment divisées (2/1).

Fui 13. - Bractée florale vue par la face dorsale (5/1).

Fig. 14. - Bractée florale vue par la face ventrale $(5,1)$.

FIG. 15. - Bouton (5/1).

Fig. 16. - Fleur épanouie $(6 / 1)$.

Firi. 17. - Lobes du périanthe avec une etamine vue de lace (9/1).

Fig. 18. - Ovaire el base du style $(4,1)$.

FiG. 19. - Coupe longitudinale le l'ovaire (18/1).

Fis. 20. - Extrémilé du style avec stigmale (9/1). 
floraison. Périanllıe rouge, glabre, d'environ $12 \mathrm{~mm}$. de long, ; segments ulu tiers environ de la longuenr du périanthe ; étanine à connectif' prolongé au delà des loges par un globule. Ovaire ovoüde allongé, densément velı, style grôle, cylindrique, glabre, à stignmate fusiforme, rétréci vers le milieu et légèrement cilié le lonğ de 4 lignes longitudinales.

\footnotetext{
Hab. - District Murray (Pritzel); Swan River (Drummond); Church Grant (Preiss); Gordon et Cauning river (Old field).
}

Observations. - L'Isopogon asper fait partic de la scconte section, celle des Eustrobilus; en effet, les écailles du còne sont nettement élargies vers le sommet comme le montrent les figures 13 et 1.1 que l'on peut comprarer avec les figures 3 et 4 de la mème planche et qui repr'ésentent les bractées de l'1. Uuxifolius de la section IIypsanthus. On peut considérer dans cette section deux subdivisions principales, celle dans laruelle le périanthe est soyeux pubescent ou villeux sur sa face extérieme, et celle dans laquelle le périanthe est glabre. Le premier de ces groupements ne nous intéresse pas pour le monent, nous aurons à l'étudier en examinant les espèces figurées planclie XXIV, qui toutes ont le périanthe velu, tandis qu'il est glabre dans notre plante comme le montrent en par-ticulier les figures 15 et 16 de la planche XX111.

Parmi les espèces caractérisées ainsi par' l'absence de villosité sur le périanthe, du moins sur le tube et la partie médiane des lobes, on pent encore constituer deux groupements qui, tout en étant géographiques, sont aussi systématiques. Les espèces orientales : I. petiolaris Cumn., I. anemonifolius linight et $l$. ceratophyllus $R$. Br., se caractérisent par les segments foliaires aplatis aigus et par lcur's lleur's jaunes; les espèces occidentales : I. asper, I. crithmifolius F. v. M., I. formosus $\mathrm{R}$. Br., I. divergens $\mathrm{R}$. Br. et $I$. scubriusculus Meissn., sont caractérisées par les segments foliaircs étroits ou presque cylindriques, mais possédant tonjour's au moins une rainure médiane et par leurs fleur's ronges ou lilas.

L'I. asper se différ'encie assez facilement des for'mes du mème groupe : en effet, ses feuilles sont pennées, ce qui se présente encore chrc\% l' $I$. divergens dont les feuilles sont même parfois doublement pennées. Mais tandis que chez la première de ces deux espèces lcs feuilles mesurent rarement beaucoup plus de $2,5 \mathrm{~cm}$. de long, chez la seconde elles mesurent rarement moins de $7,5 \mathrm{~cm}$. et souvent plus de $10 \mathrm{~cm}$.

La plante figurée a été recueillie par M. Pritzel dans le district Murray, dans les forêts sablonneuses, en août 1901 (Pl. Austral. occid., ฉ. 481$)$. 


\section{Isopogon tripartitus $R . B r$.}

Prot. Novæ (1830) p. 8; Meisn. in Pl. Preiss. II n. 247 et in DC. Prod. regn. veget. XIV p. 280 ; Benth. Fl. Austral. V p. 344.

Isopogon trilobus Meisn. in Lehm. Pl. Preiss. I (1838) p. $507 ; F . v$. Muell. Fragm. VI p) 239.

Petit arbrisseau de $60 \mathrm{~cm}$. à 1 mètre de hauteur, glabre, sauf dans ses inflorescences. Feuilles longuement pétiolées, profondément trilobées, à lobe médian souvent trilobulé, à segments aigus, plus ou moins nettement striés longitudinalement. Inflorescences terminales sessiles, ovoïdes ou globuleuses. Bractées externes peu nombreuses, plus courtes que les écailles florales. Écailles florales ovalesaiguës, acuminées, plus ou moins cucullées, glabres sur la face interne, densément velues jusqu'au sommet sur la face externe. Périanthe glabre dans son tiers inférieur, velu dans les autres parties, à 4 lobes atteignant le tiers de la longueur totale. Étamine à connectif terminé au soinmet par un globule. Ovaire ovoïde, densément velu; style grêle ailé, terminé par un stigmate ovoïle, cilié le long de lignes plus ou moins nettes.

Hab. - District North-West Plantagenet (Pritzel); King George's Sound (Baxter et Drummond) : au nord du Stirling Range (F.v. Muell.).

Explication des figures 1-9 de la planche XXIV.

Isopogon tripartitus $R$. $B r$.

Hig. 1. - Rameau fleuri et fructifère (1/1).

Fig. 2. - Bractée florifère vue de profil (4/1).

FIG. 3. - Bractée florifère vue de face (4/1).

Fig. 4. - Fleur épanouie (6/1).

Fig. 5. - Extrémité dun des lobes du périanthe avec une anthère vue de fare $(8 / 1)$.

F16. 6. - Ovaire, style et stigmate (6/1).

Flg 7. - Coupe longitudinale de l'ovaire (20/1).

FIG. 8. - Style terminé par le stigmate séparé de l'ovaire (6/1).

F1G. 9. - Stigmate (12/1). 
Observations. - L'l. lripartitus appurticnt, comme l'espèce que nous venons d'examiner auparavant, à la section fiustrobilus : ses bractées (fig. 3) sont nettcment élargics vers le sommet. Mais tandis que l'I. asper se classait dans le sous-groupe ì périanthe glabre, l'espèce que nous examinous ici, se range dans le sous-groupe à fleurs à périanthe velu, comme nous le inontre la figure 4 de la planche ci-jointe.

Les neuf cspèces commues actuellement dans ce groupement ( 8 espèces de la F'lora australiensis de Bentham et l'I. alcicornis Diels in F'ragm. Phyt. Austral occid. p. 134, fig. 1:3) peuvent se répartir' en deux subdivisions ell se basant sur la forme des segments foliaires. Le plus grand nombre possède des feuilles à segments aplatis, les trois autres : $I$. trilobus R. Br., I. tripartitus R. Br. et $I$ longifolius R. Br. ont des fenilles à segments aplatis.

La différenciation de ces trois plantcs n'est pas difficile à établir : en effet, dans la première l'entre elles les feuilles sont tridentées ou courtement trilobées; :he\% la deuxième les feuilles sont divisées généralement en 3 scgments variant de 12 à 25 cul. de long, parfois le segment nédian bi- ou trilobulé; chez la troisième espèce les fenilles linéaires on obancéolées et mesurant jusque $20 \mathrm{~cm}$. de long, sont entières ou bi- ou trilobées à lobes linéaires. La détermination de l'espèce ne peut donc présenter le moindre doute.

La plante qui a servi de modèle pour les analyses des figures 1 à 9 de la planche XXIV, provient du I)istrict North-West Plantagenet, où elle a été récoltée en novembre 1901, dans une zone sablouneuse, par M. E. Pritzel et a été distribuée sons le $11^{\circ} 95^{\circ} 2$ de ses Plantce Australice occidentalis.

\section{Isopogon teretifolius $R$. $B r$.}

in Traus. Linn. Soc. X (1810) p. 71 et Prod. p. 365; Meisn. in Fl. Preiss. I p. 504 et in DC. Prod. regul. veget. XIV p. 277; F. v. Muell. Fragm. VI p. 241 ; Benth. Fl. Austral. V p. 346 ; Diels et Pritzel Fragm. Phyt. Austral. occid. p. 136.

Isopogon petrophiloides $R$. Br. Prot. nov. (1830) p. 7. Isopogon cornigerus Lindl. Swan River $\Lambda$ pp. (1840) p. 34.

Arbrisseau de $60 \mathrm{~cm}$. à $1 \mathrm{~m} .25$ de haut, à ramifications jeunes pubescentes-soyeuses, devenant glabres avec l'âge. Feuilles cylindriques, entières, fourchues ou divisées 2 à 3 fois, atteignant $7,5 \mathrm{~cm}$. de long, à segments divariqués, 
aigus au sommet. Inflorescences terminales, déprimées, globuleuses, à bractées externes subobtuses plus courtes que les internes. Écailles florales ohovales-spatulées, glabres à la base et sur toute la face interne, densément velues sur la face externe, apiculées au sommet. Périanthe rose ou blanchâtre, d'environ $12 \mathrm{~mm}$. de long, glabre dans son tiers inférieur, velu, sur le reste, à poils assez longs au sommet; segments du tiers environ de la longueur du périanthe. Étamines à connectif prolongé au delà des loges en une sorte de globule. Ovaire ovoïde, longuement velu; style orangé, grêle, glabre, cylindrique, terminé par un stigmate fusiforme, courtement cilié le long de 4 lignes proéminentes, contracté et renflé vers le milieu, glabre dans sa partie supérieure

Hab - District du North West Plantagenet (Pritzel : King George : Sound (R. Brown et autres): à lest des Monts Barren (Maxwell); au nord des plaines de Quangen (Preiss); vers la Swan River (Drummond).

Observations. - L'I teretifolius appartient, comme l'I. tripartitus dont nous venons d'étudier sommairement les caractères, à la section des Eustrobilus et au groupement secondaire des Isopogon à périanthe velu (cf. fig. 4) Mais ses feuilles à segments cylindriques le font classer en opposition avec l'I. tripartitus parmi les six espèces dont nous avons signalé la présence plus haut et qui justement sont caractérisées par les feuilles ou leur's segments cylindriques. De ces espèces, les quatre pre-

\section{Explication des figures 10-18 de la planche XXIV.}

Isopogon teretifolius $R . B r$.

FIG. 10. - Extrémité d'un rameau florifère (1/1).

Fig. 11. - Bractée florale vue de dos (6/1).

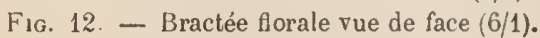

FIG. 13. - Fleur avant son épanouissement complet (6/1).

FIg. 14. - Fleur épanouie, (\$/1).

Fig. 15. - Extrémité d'un des lobes du périanthe arec une étamine vue de face $(9 / 1)$.

FiG. 16. - Ovaire avec la base du slyle (11/1).

Fra. 17. - Coupe longitudinale de l'ovaire (25/1).

F1G. 18. - Extrémité du style et stigmate (11/1). 
mières (I. Drummondii Benth., I. heterophyllus Meisu., I. villosus Meisn. et $I$. alcicornis Diels) sont earactérisées par leurs braetées oxtérieures petites et parfois peu visibles; les deux autres, soit l'I. teretifolius R. Br', que nous examinons, et l'I. anethifolius Kinight, possèdent des braetées externes stériles assez développées et glabres; la figure 10 de notre planche $X X 1 Y$ permet de se rendre eompte de ee caractinc.

'Tandis quo l' $I$. ancthifolins appartient à la flore de l'Australie orientale, l'I. teretifolius appartient à eelle de l'Australie occidentale. Parmi les earaetères différentiels nous pouvons encore eiter le réeeptaele qui est cylindrique chez l'I anethifolins, et eonique chez l'I. teretifolius.

La plante dont nous donnons quelques analyses. provient du Stirling liange (N. W. Plantagenet) où elle a été recucillie en septembre 1001 par M. Pritzel; elle a été publiée par lui sous le $n^{0} 704$ dans ses Planta Australive occidentalis.

\section{Isopogon Drummondii Benth.}

in Fl. Austral. V (1870) p. 344.

Isopogon petrophiloides Meisn. p. p., (non lir.), in Pl. Preiss. I (1844) p. 503 et in $D C$. Prod, regn. veget. XIV p. 276 .

Arbrisseau atteignant $1,5 \mathrm{~cm}$. de haut, à rameaux jeunos pubescents, devenant glabres avec l'àge. Feuilles cylindriques, simples, aiguës au sommet, atteignant $7 \mathrm{~cm}$. long. Inflorescences terminales, dépassées par les feuilles

\section{Explication des figures $19-26$ de la planche XXIV.}

\section{Isopogon Drummondii Benth.}

FIG. 19. - Extrémilé d'un rameau florifère (1/1).

FIG. 20. - Bractée florale vue par la face interne (5/1)

FIG. 21. - Bractée florale vue par la face externe $(5 / 1)$.

Fıg. 22. - Fleur épancuie (6/1).

Fıg. 23. - Estrémité dun des lobes du périanthe avec une étamine vue de face (11/1).

FIG. 24. - Ovaire, style et stigmate (6/1).

Fis. 25. - Coupe longitudinale de l'ovaire (25/1).

FIg. 26. - Extremité du style et stigmate (12/1). 
a.voisinantes; cônes sessiles. subg̣lohulenx. Bractées externes obtuses, plus courtes que les bractées internes. F́cailles florales ovales, aiguës, densément velues sur la face extérieure, glabres sur la face interne. Périanthe atteignant environ $8 \mathrm{~mm}$. de long, à segments du tiers environ de la longueur du périanthe; segments glabres légèrement velus sur leur face externe. Étamines à connectif prolongé au delà des loges en une sorte de globule. Ovaire ovoïde, densément velu; style grrêle, cylindrique, glahre, à stigmate fusiforme, plus ou moins quadrangulaire à la hase légèrement papilleux-puhescent, rerflé dans sa partie intermédiaire, glabre et obscurément quadrangulaire vers le sommet.

\section{Hab. - District Murray (Pritzel): Swan River (Drummond).}

Observations. - L'I. Drummondii, dédié à l'un de ceux qui ont le plus fait four la connaissance de la Flore de l'Australie occidentale, appartient. d'après la Flora australiensis do Bentham, au même sous-groupe que l' $I$. teretifolius dont nous venons de donner les affinités. Cependant une des figures analytiquues de notı'e planche (fig. 20). ne cadıe pas totalement avec les caractères que doivent posséder les Isopogon de la section Eustrobilus, c'est-à-dire des bractées élargies ou tronquées au scmmet. Sauf ce caractère qui n'a peut être pas autant de valeur que ne lui en accordent les anteur's, les analyses de notre planche permettent de rapporter notre plante au groupe des Isopogon dont l'inflniescence ne possède que des bractées réduites et des écailles florifères velues. dont nous avons énuméré ci-avant les quatre espèces.

De ces quatre espèces, les deux premières seules (I. Drummondii et I. heterophyllus\} peuvent se présenter avec des feuilles simples et encore ces dernières ne sont-elles la rrigle que dans la première des deux seulement, l'I. heterophyllus présentant souvent des feuilles bi- ou trilobées; ell outre tandis que l'inflorescence forme un cône ovoïdeglobuleux chez la dernière espèce, elle est toujour's déprimée-globuleuse chez l'I. Drummondii comme le fait roir d'ailleur's très bien la figur' 19 de la planche XXIV. L'on ne pourrait pousser plus avant la définition des caractères différentiels de ces deux espèces, le périanthe de l'I. heterophyllus n'ayant pas été décrit.

Le type qui a servi au dessinateur', provient des forêts sallonneuses du district de Murray (Planı Australie occidentalis, n²69. 
Nily
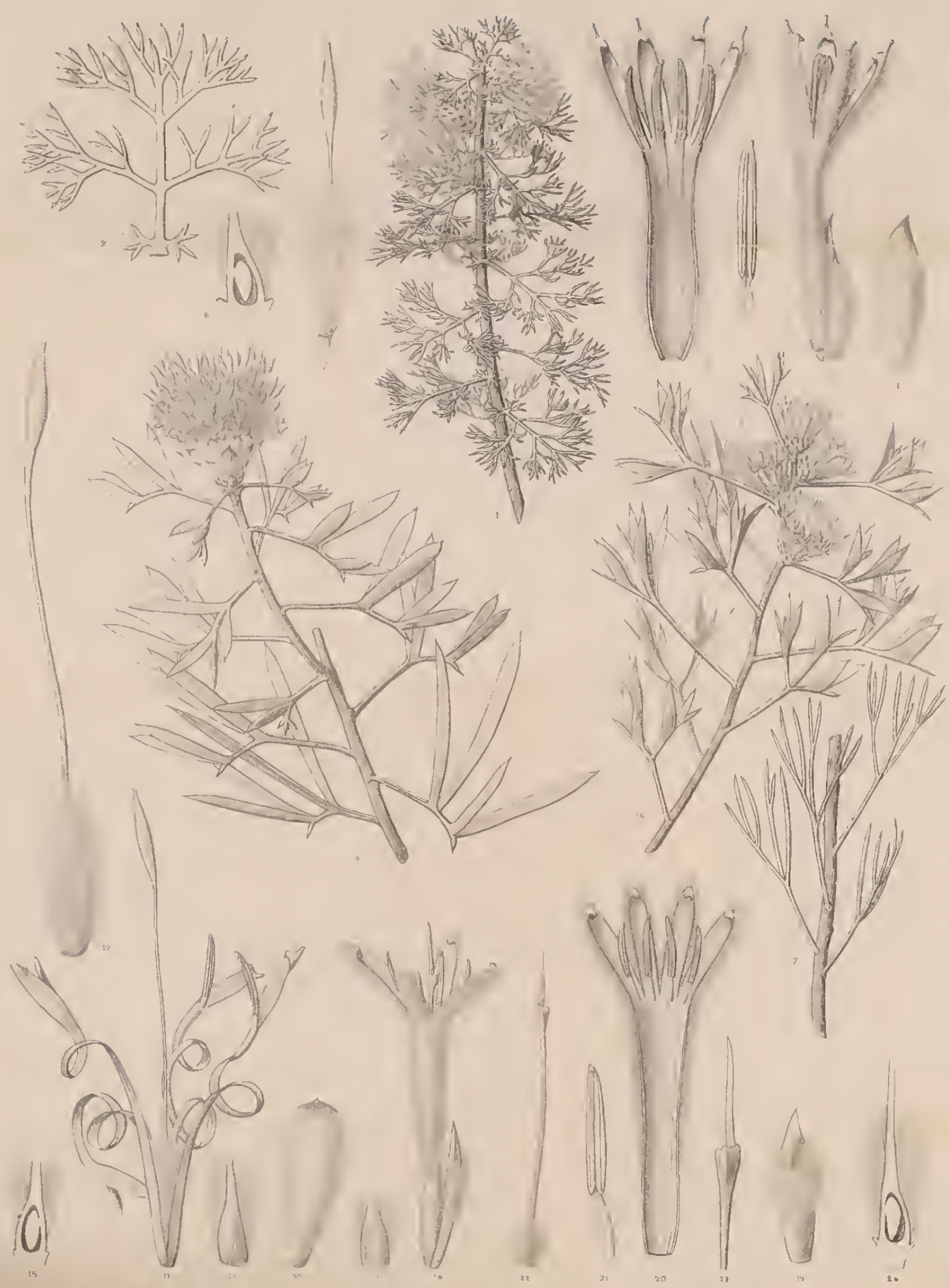


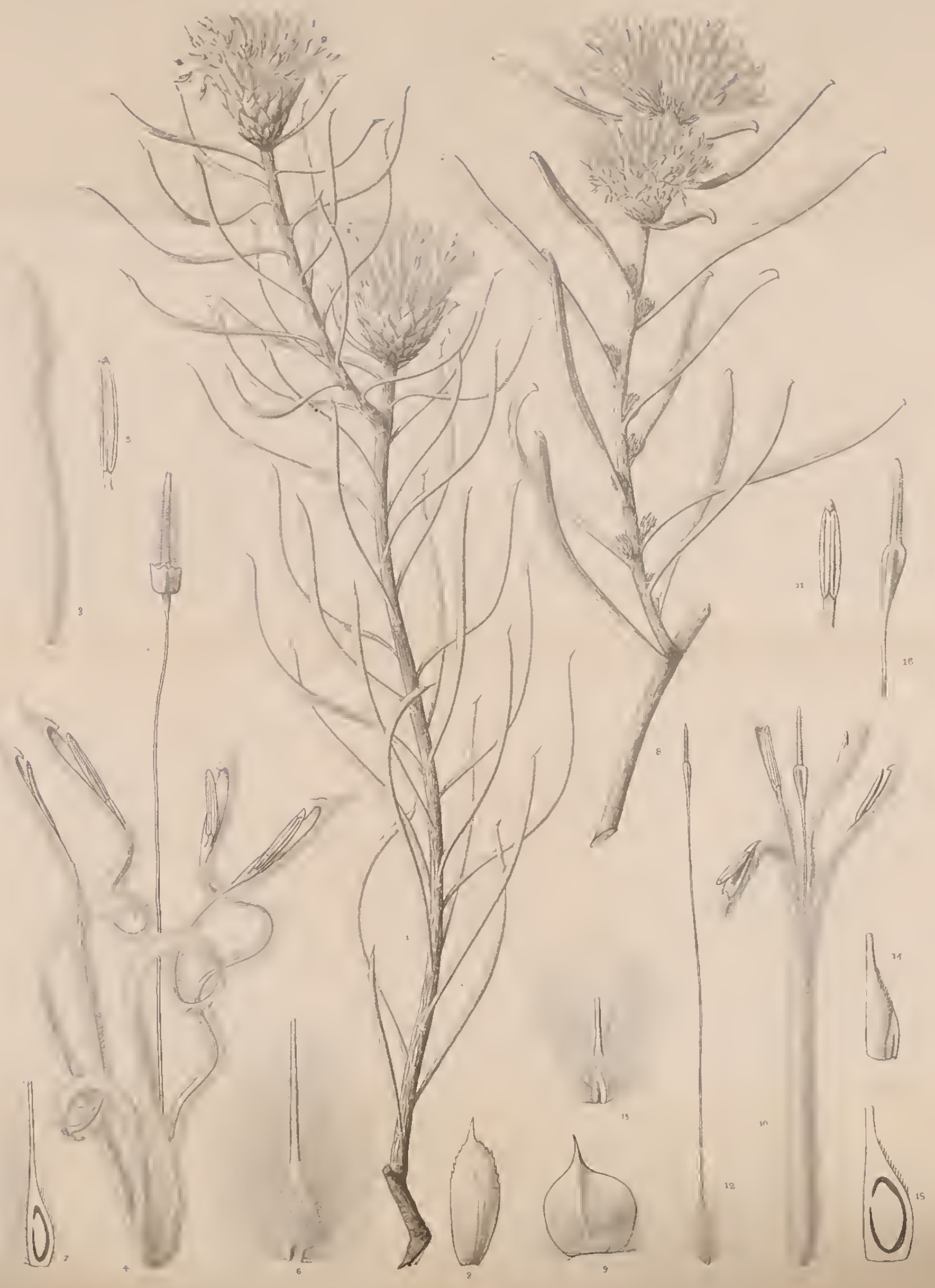




\section{PETROPHILA R. Br.}

Ce gemre fut créé en 1810 par $\mathrm{k}$ Brown dans les 'Transactions de li Société Linnécnne de Londres. Il est composé de plus de 30 espèces dont le plus grand nombre est localisé dans l'. Iustralie oceidentale. I)epuis la publication de la Flore d'Iustralie, l'étude des matérianx nouveanx rapportés par les voyageurs, n’a guère amené la découverte de nombreuses espèces nouvelles; le baron F. v. Mueller renseigne dans son Census of Australian plants, 37 espèces dont certaines sont considérées eomme simples variétés.

Les recherches floristiques de MM. Diels et Pritzel ont confirmé eomplétement les résultats obtenus antérieurement, à savoir que les Petrophila sont particulièrement répandus en Australie oeeidentale, mais qu'ils manquent totalement dans cette région intérieure du pays qu'ils ont qualifié d'Eremaea. Les formes du genre paraissent alfectionner particulièrement des sols ferrugineux et préfèrent un fort éclairage.

Les Petrophila sont caractérisés par des fleurs blanches ou jaunâtres disposées en épis denses ou sortes de eònes, elıacune des fleur's composantes étant sessile à l'aisselle d'une bractée écailleuse; ces cônes sont de for'me variable et leur's écailles dureissent après floraison et deviennent assez fortement imbriquées. Les fleurs sont hermaphrodites, ì périanthe régulier, à tube assez grêle formé te 4 segments qui parfois restent réunis à la base, parfois se séparent totalement. Les 4 anthères opposées aux lobes du limbe sont toutes fertiles. L'ovaire est sesssile, à un, rarement deux ovules, et non muni d'écailles hypogynes; il est terminé par un style toujour's filiforme dont l'extrémité est assez variable; e'est sur les formes que prend cette dernière que sont basés, en partie du moins, les caractères des sections proposées par Bentham et sur lesquelles nous reviendrons plus loin. Le fruit est une sorte de noix indéhiscente, plus ou moins comprimée, parfois ailée, à longs poils le long des côtés ou à partir de la base, parfois totalement velue.

Le genre Petrophila est, comme nous l'avons dit, très voisin du genre Isopogon.

Les 6 sections dans lesquelles se répartissent les espèees du gemre sont caractérisées comme suit : 
Section I. - Avthrostigma: Feuilles non divisées; cônes terminaux, g'ćnéralement assez grands; style épaissi et tronqué cn dessons du prolongement villeux; ou velu cylindrique. Segments du rérianthe se séparant jusqu’à la base.

Section 1I. - Yerostele : Feuilles planes divisées en trois lobes, l'arement entières; cônes axillaires, ovoïdes; style épaissi et généralement tronqué sous le prolongement, grêle et presque glabı'e. Tube du périanthe grêle, tombant généralement en une fois.

Section III. - Serrurioides : Feuilles divisées à segments cylindriques ou aplatis; cônes axillaires ovoïdes. Périanthe à tube grôle, tombant en une fois. Style continu terminé en un renflement fusiforme.

Section IV - Symphyolepis : Feuilles planes, lobées ou divisées; cônes axillaires et rarement terminaux. Périanthe à segments se, séparant généralement successivement. Style continu terminé en un renflement fusiforme.

Section V. - Petrophyle : Feuilles divisées, rarement simples, i segments cylindriques ou étroits et aplatis; cônes terminaux ou axillaires. Périanthe à segments se séparant généralement successivement. Style continu terminé par un renflement fusiforme.

Section VI. - Hebegyne: Feuilles cylindriques, simples ou biou trilobées vers le sommet. Cônes terminaux ou dans les aisselles des ramifications. Périanthe à segments se séparant successivement. Style pubescent, renflé ver's le sommet mais légèrement fusiforme.

Les cinq espèces que nous figurons ci-après se classent dans les quatre premières sections, qui peuvent facilement se différencier en se basant sur la disposition des cônes, sur la séparation des lobes du périanthe et sur la forme du style. La section Hebegyne assez voisine des Petrophyle ne comprend qu'une seule espèce, le $P$. semifurcata F. $\nabla$. Muell. de la Murchison River; la section Petrophyle, par contre, renferme 15 espèces et on pourrait parfois considérer comme appartenant à cette section les P. Shuttleworthiana et macrostachya de la section Symphyolepis dont nous aurons à examiner les caractères en décrivant la première d'entre elles. En effet, Petrophyle et Symphyolepis ont de très nombreux caractères communs et la comparaison des diagnoses de ces deux sections, que nous avons rappelées plus haut, prouve bien, comme on l'a fait ressortir parfois, l'affinité très grande des trois delnières sections du genre. 


\section{Petrophila megalostegia $F, v$. Muell.}

Fragm. phytographie Australix X (1876-1877) p. 61; Diels et I'ritzel Fragm. Phyt. Australia occid. p. 132.

Plante glabre dans toutes ses parties sauf sur celles de l'inflorescence. Feuilles cylindriques, entières, rétrécies à la base en une sorte de pétiole, terminées au sommet en un imucron très piquant. Inflorescences terminant les rameaux ou leurs ramifications, formant des cônes suhsessilles, subglobuleux. Bractées involucrales glabres; bractées florales glabres, ovales lancéolées, aiguës, denticulées sur les bords, striées sur le dos. Périanthe densément velu, se séparant jusqu'à la base en 4 segments. Étamines logées à la partie supérieure des segments, à deux loges parallèles, à connectif dépassant légèrement les loges. Ovaire ovoïde, plus densément velı sur la face dorsale que sur la face ventrale, terminé par un style grêle, cylindrique, glabre, brusquement élargi en une sorte de renflement tronqué d'oì émerge le stigmate cylindrique, velu, à poils étalés. Fruit rhomboédrique-arrondi, veluséricé sur la face externe, muni de poils allongés sur la face interne.

Hab. - Entre les fleuves Moore et Murchison (Pritzel); sans indication de localité (Drummond); district Irwin près Mingenew et près de la rivière Green wigh (Diels).

Explication des figures 1-7 de la planche XXV.

Petrophila megalostegia $v$. Muell.

Fig. 1. - Rameau fleuri (1/1).

Fıo. 2. - Bractées de linflorescence vues de dis (4/1).

FIG. 3. - Bouton (4/1).

Fia. 4. - Fleur èpanouie (6/1).

Fra. 5. - Étamines vues de face (10/1).

Fig. 6. - Ovaire vu par la face antérieure (10/i).

Fra. 7. - Coupe antéropostérieure de lovaire (10/1). 
Obscrvations. - Ainsi qu'on peut le voir en jetant un coup d'œil sur les figures 1-7 de la planche XXV, le $P$. megalostegia F. v. Muell. se range très clairement dans la section Arthostigma. Dans cette der'nière Bentham comprenait cing espèces; le baron von Mueller en admet sept, la dernière n'étant pour Bentham qu'une variété de celle qui la précède dans la liste ci-dessous, où nous avons indiqué la distribution générale de ces plantes :

\begin{tabular}{|c|c|c|}
\hline P. leretifolia R. $\mathrm{Br}^{\circ}$. & \multicolumn{2}{|c|}{ Australie ocridentale } \\
\hline P. longifolia $\mathrm{R} . \mathrm{Br}^{\mathrm{P}}$. & $"$ & n \\
\hline P. media R. Br. & " & " \\
\hline$P$. acicularis $\mathrm{R}, \mathrm{Bl}^{\circ}$. & n & " \\
\hline P. megalostegia F. v. Muell. & , & - \\
\hline$P$. linearis $\mathrm{R} . \mathrm{Bl}$. & $"$ & " \\
\hline$P$. anceps $\mathrm{R} . \mathrm{Br}$. & . & " \\
\hline
\end{tabular}

De ces sept espèces les deux dernières seules possèdent des feuilles aplaties; toutes les autres les ont cylindriques comme le fait fort bien ressortir la figure I de la planche $\mathbf{X X V}$. Les trois premières espèces citées se caractérisent entre autres par l'absence de stries sur' les ćcailles du còne, tandis que chez les $P$. acicularis et megaslostegia les écailles sont striées longitudinalement. Ces deux Petrophila sont cer'tainement affines; cependant si l'on compare notre planche avec celle du $P$. acicularis, qui a paru dans le Botanical Magazine (tah. :3.1i9), on verra qu il est assez aisé de différencier ces deux espèces en se basant sur la longueur des feuilles dépassant le capitule floral chez le $P^{\prime}$. acicularis, sous la fol'me de bractées florales ovales, longuement acuminées, au lieu d'ètre ovales-oblongues courtement aiguës dans le $P$. megalostegia, comme le montre la figure 2 de notre planche $\mathrm{XXV}$.

La plante figurée a été récoltée dans les régions sablonneuses entre les fleuves Moore et Murchison, par M le Dr E. Pritzel en septembre 1901 , et porte le $n^{\circ} 645$ dans ses Plantw Australice occidentalis.

\section{Petrophila linearis $R . B r$.}

Prot. Nove (18:30) p 6; Mcisn. in Pl. Preiss. I 1. 494 et in $D C$. Prod. regn. veget. XIV p 267 : Benth. Fl. Austral. V p. 323; $F$. v. Mucll. Fragm. phyt Austral. VI p. 243; Diels et Pritzel Fragm. phyt. Austral. occid. p. 132.

Arbrissean dresse atteignant is cm., glabre et d'un vert glaucescent sur toutes ses partics saul sur les inflores- 
cences. Fenilles linéaires, plus on moins aplaties mais épaisses, de 2 à $6 \mathrm{~mm}$. de diamètre dans leur partie médiane, et de $3,5 \mathrm{~cm}$. ¿ $10 \mathrm{~cm}$ de long, rétrécies vers la base en une sorte de pétiole cylindrique, généralement recourbées au sommet en forme de liameçon, subobtuses, à mucron droit ou plus ou moins recourbé. Inflorescences terminant les rameaux principaux à leurs ramifications, formant des cônes ovoïdes ou subglobuleux, sessiles de 12 ì $20 \mathrm{~mm}$. de diamètre, entourés de bractées largement ovales, aiguës, les intérieures les plus développées, non ciliées sauf à la base, glabres ou plus ou moins striées a vec l'âge. Périanthe rosé atteignant $2,5 \mathrm{~cm}$. de long, densément villeux, à poils soyeux, blanchâtres ou lerrugineux, ì segments glabres sur la face interne, se détachant séparément après l'anthèse, muni au sommet d'un r'enflement et d'un épaississement en crochet. Étamines logées vers le sommet des segments du périanthe, à connectif dépassant légèrement les loges, à loges parallèles à fente longitudinale. Ovaire ovoïde, fortement velu sur la face dorsale, moins velu sur la face ventrale, à style allongé, grêle, aussi long que les segments de périanthe, insensiblement élargi, quadrangulaire terminé par une sorte de cupule de laquelle émerge le stigmate cylindrique, écarlate, velu, à poils jaunâtres, courts, étalés. Fruit aplati, à bords ciliés de longs poils, à face interne villeuse, à face externe glabre.

\section{Explication des figures 8-16 de la planche XXV.}

Petrophila linearis $R$. $B r$.

F1G. 8. - Rameau florifère (1/1).

Fio. 9. - Bractée de l'inflorescence (5/1).

F10. 10. - Fleur épanouie (5/1).

Fio. 11. - Étamine vue de face $(6 / 1)$.

Fio. 12. - Ovaire, style et stigmate (4/1).

Fı. 13. - Ovaire vu par la face antérieure (6/1).

Fig. 14. - Ovaire vu latéralement (10/1).

Fio. 15. - Coupe antéro-postérieure de l'ovaire (14/1).

FIG. 16. - Extrémité lu style avec stigmate (10/1). 


\section{Hab. - Distcict Swan (Pritzel); Vasse River et Swan River (Fraser, Drummond et Oldfield .}

Observations. - Le $P$. linearis $\mathrm{R}$. Br. forme avec le $P$. anceps $\mathrm{R}$. $\mathrm{Br}$. que Bentham considérait dans sa Flora Australiensis comme une variété, un groupe particulier parmi les Arthrostigma que nous avons énumérés à propos de l'espèce précédente. Il suffira de jeter un coup d'œil sur les figures de la planche XXV pour juger des différences de ces deux espèces; en effet, les feuilles se présentent chez le $P$. linearis comme aplaties, tandis qu'elles sont cylindriques chez le $P$. megalostegia et les espèces voisines.

Les caractères qui permettent de séparel le $P$. linearis du $P$. anceps résident dans les feuilles de cette dernière espèce, qui sont plus minces; les inflorescences et leur's parties constituantes sont également plus réduites. I ،a dispersion du $P$. anceps, parait un peu différente de celle du $P$. linearis, il n'a été signalé que dans la région du King George's Sound et au pied du Stirling Range.

La plante figurée a été recueillie dans les forêts sablonueuses et peu compactes du Swan district sous les Eucalyptus marginatus (Pritzel Pl. Austral. occid. n. 1, novemb. I900).

L'espèce parait d'ailleurs assez répandue dans toute la zone littorale de l'Australie occidentale, jusqu'i la Rivière Moore; elle fleurit en octobre et novembre.

\section{Petrophila Serruriæ R. Br.}

Prot. Nov. (I830) p. 6; Meisn. in Pl. Preiss. I p. 497 et DC. Prod. regn. veget. XIV p. 271 ; Benth. Pl. Austral. V p. 327.

Petrophila glanduligera Lindl. Swan River App. (1840) p. 35); Meisn. loc. cit. p. 498 et 271 .

Petrophila axillaris Meisn. in Hook. Lew Joulll. VII (1855) p.68 et in $D C$. loc. cit. p. 275.

Plante buissonnante, dressée, atteignant un peu plus de 1 mètre de haut, à rameaux âgés glabres ou glabrescents, plus ou moins velus à l'état jeune. Feuilles relativement petites, atteignant, au maximum, $3 \mathrm{~cm}$. de long, rassemblées surtout vers les extrémités des rameaux, bi- ou tripennées, à segments inférieurs très rapprochés de la base et notablement moins développés que les segments médians; segments moyens grêles, velus au moins à l'état jeune, cylindriques, plus ou moins sillonnés sur la face su- 
périeure, divariqués, aigus-acuminés au sommet. Inflorescences en cônes ovoïdes ou subglobuleux, sessiles ou très courtement pédicellés, localisés dans les aisselles des feuilles supérieures, parfois très rapprochés, formant des fascicules terminaux ou suhterminaux. Bractées florales ovales, aiguës, glabres au sommet mais densément velues à la base. Périanthe grêle, soyeux ou ferrugineux-villeux, atteignant $12 \mathrm{~mm}$. de long, se détachant en une fois après l'anthèse, à lobes un peu plus courts que le tube, épaissis au sommet, à extrémités terminées par un acumen grêle portant une glande terminale disparaissant parfois parmi les poils Ovaire ovoïde, densément velu, style grêle terminé par un stigmate fusiforme, à poils plus ou moins, réfléchis, disposés en rangées longitudinales. Fruit ovoïdeaigu, glabre sur le dos, à face ventrale et bords munis de longs poils.

$\mathrm{Hab}$ - King George's Sound et districts voisins jusqu'aux Vasse et Swan Rivers (divers): entre les Moore et Murchison Rivers (Drummond et Pritzel).

Observations. -- Le P. Serrurice appartient à la section Serrurioides que nous avons caractérisée dans les quelques lignes consacrées au genre Petrophil\%. Dans cette section se classent:

$P$. divaricala R. Br.

$P$. Serrurice $\mathrm{R}$. Br.

$P$. inconspicua Meisn.

$P$. trifida R. Br.

qui tous les quatre sont localisés dans l'Australie occidentale.

Explication des figures 1-8 de la planche XXVI.

Petrophila Serruriæ $R$. $B r$.

Fig. 1. - Rameau fleuri (1/1).

Fro. 2 - Feuille vue de face (2/1).

Fıo. 3. - Fleur avec sa bractée (6/1).

Fig. 4. - Bractée isolée vue de dos (6/1).

FıG. 5. - Corolle fendue et étalée (6/1).

Fia. 6. - Étamine (12/1).

FIg. 7. - Ovaire, style et stigmate (6/1).

Fir. 8. - Coupe longitudinale de l'ovaire (20/1). 
Ia dimension des feuilles permet d'établir dans ces quatre plantes trois groupements :

Feuilles de 5 à $7,5 \mathrm{~cm}$. de diamètre . . . . P. divaricata.

Feuilles de $12 \mathrm{~mm}$. à $2,5 \mathrm{~cm}$. environ de

long . . . . . P. Serruria et $P$ inconspicua.

Feuilles de $\bar{y}$ à $10 \mathrm{~cm}$. de long . . . . P. trifida.

Ces caractères suffisent pour faire voir que la plante que nous avons fait figurer (pl. XXV1, fig. 1-8) ne pent ètre que le $P$. Scrvurice ou le P. inconspicua.

La clef analytique proposée par Bentham dans la Flore d'Australie se base pour différencier ces deux espèces sur la disposition des segments foliaires. Ceux-ci sont serrés, dressés, non aigus et piquants au sommet chez le $P$. inconspicua, tandis qu'ils sont dressés et divariqués, beaucoup moins compacts chez le $P$. Sermurice et en général terminés prar use pointe, comme le montre la figure 2 de la planche XXVI.

La grandeur des feuilles ajoute encore un bon caractère distinctif; tandis que chez la plante que nous figurons ici, les feuilles environ aussi longues que larges atteignent dans les deux dimensions $2,5 \mathrm{~cm}$. environ, chez le $P$. inconspicua elles seraient beaucoup plus petites et ne mesureraient guère que $12 \mathrm{~mm}$. dans les deux sens.

La plante qui a servi ¿ faire les analy'ses des figures 1-8 de la planche XXVI, a été recueillie par M. Pritzel en août 1904 dans les régions sèches de la région de la rivière Moore (Plantoe Austral. occid., n. 577). M. Pritzel a ohservé des coloris différents dans les lleur's, suivant les r'égions d'oì elles provenaient; c’est ainsi que dans les régions sèches on rencontre souvent la plante avec des fleurs jaunes, et Jans les régions calcaires du littoral de l' $\Lambda$ ustralie occidentale les fleurs sont généralement colorées ou roses.

\section{Petrophila Shuttleworthiana Meisn.}

in Pl. Preiss. (184.1) 1) 246 et in DC. Prod. regn. veget. XIV p. 275; Benth. Fl. Austral. V p. 329.

Plante buissonnante, à rameaux rigides glabres. Feuilles pétiolées, à pétiole canaliculé, légèrement aplati, pennées à segments linéaires ou lancéolés, entiers ou bi- ou trifides, très rigides, aigus, à acumen pointı. Inflorescences terminales ou axillaires vers le sommet des rameaux, oblongues 
ou subeylindriques. Bractées externes courtes, caduques; écailles ou bractées du cône, obovales, obtuses-acuminées au sommet, densément velues sur la face extérieure, glahres sur la face interne. Périanthe jaune ou rosé, glahre, de $8-10 \mathrm{~mm}$. de long, à segments se séparant jusqu'à la hase et s'enlevant séparément. Ovaire ovoüde, densément velu; style grôle, cylindrique, plusieurs fois plus long que l'ovaire, à stigmate fusiforme éparsement et courtement velu. Fruit non ailé, à bords aigus, à face dorsale et bords velus, à face ventrale glabre.

\section{Hab. - Moore River (Pritzel): Murchison River (Oldfield).}

Observations. - Le P. Shuttleworthiana appartient lui à la section 4, Symphyolepis, caractérisée, comme nous l'avons vu plus haut, par ses feuilles planes, lobées ou divisées profondément, ses inflorescences axillaires, rarement terminales, son style continu et fusiforme.

Cette section renferme peu d'espèces, on y trouve :
$P$. carduacea Meisn.
$P$. Shuttleworthiana Meisn.
P. macrostachya R. Br.
$P$. diversifolia $\mathrm{R}$. Br.

Ces quatre espèces sont localisées dans l'Australie occidentale; leur's caractères différentiels sont très tranchés : en effet, la première a des feuilles sessiles, pennatifides; elle ne peut done etre confondue avec la plante que nous figurons et dont les feuilles sont nettement pédicellées, ce qui est le cas d'ailleurs pour les autres espèces de la section. Il sera facile d'écarter de notre examen le $P$. diversifolia dont le périanthe

\section{Explication des figures 9-15 de la planche XXVI.}

Petrophila Shuttleworthiana Meisı.

Fro. 9. - Rameau fleuri, (1/1).

F1G. 10. - Bractée florale vue de dos, (5/1).

Fig. 11. - Fleur épanouie, (5/1).

FIG. 12. - Ovaire, style et stigmate, $(7 / 1)$.

F16. 13 - Base de l'ovaire, (8/1).

Fig. 14. - Partie supérieure de l'ovaire, (8/1).

FIG. 15. - Coupe longitudinale de l'ovaire, $(8 / 1)$. 
est velu sur la face extérieure, tandis qu'il est glabre chez les deux e.pèces r'estantes.

Bentham base ensuite la différenciation de ces espèces sur les fruits; nucules obtuses, glabres sur la face dorsale chez le $P$. Shuttleworthiana, acuminées vers le sommet, à poils en touffes sur les deux faces chez les autres. Mais ce caractère ne peut nous servir quand nous ne possédons pas de fruits. I)es autres caractères nous en retenons un seul qui peut, semble-t-il, servir à séparer ces deux plantes dont les descriptions sont très comparables, c'est la nature des bractées florales formant le cône : elles sont densément villeuses sur le dos, glabres au sommet chez le $P$. Shuttleworthiana, comme nous le montre la figure 10 , et villeuses à la base seulement chez le $P$. macrostachya.

La plante qui nous a permis de donner ici quelques figures analytiques, provient de la Moore River et $\mathrm{y}$ a été récoltée par M. le $D^{r}$ E. Pritzel (Pl. Austral. occident., n. 729).

\section{Petrophila propinqua $R . B r$.}

Prot. Novæ (1830) p. 7; Meisn. in Pl. Preiss. I (1841) p. 501 et in DC. Prod, regn. veget. XIV p. 273 ; Benth. Fl. Austral. V p. 325.

Arbrisseau atteignant $1 \mathrm{~m} 25$ de hauteur, glabre à l'état adulte sauf sur les inflorescences; rameaux jeunes cylindriques, assez grêles et courtement pubescents. Feuilles à pétiole assez long, au moins aussi long que le limbe, divisées en 3 lobes trifides ou pennées, à pinnules

\section{Explication des figures 16-24 de la planche XXVI.}

Petrophila propinqua $R . B r$.

F1G. 16. - Extrémité d'un rameau florifère (1/1).

F1G. 17 - Base d'un rameau à feuilles à segments linéaires (1/1).

FIG. 18. - Fleur épanouie avec sa bractee (6/1).

F1G. 19. - Bractée isolée vue de dos (6/1).

Fis 20. - Corolle fendue et étalée (6/1).

Fig. 21. - Étamine vue de face (11/1).

FIG. 22. - Ovaire, style et stigmate (6/1).

FIG. 23. - Extrémité du style et stigmate (10/1).

Fig. 24. - Coupe longitudinale de l'ovaire (15/1). 
inférieures divisées; segments aplatis, linéaires-lancéolés ou linéaires-aigus, pouvant atteindre dans le dernier cas jusqu'à $3 \mathrm{~cm}$. de long. Inflorescences axillaires, ovoüdes ou subglobuleuses, sessiles, ne dépassant guère, sons les périanthes, 12 mun. de long. Bractées involucrales sur 3 rangs, les internes les plus longues, lancéolées, aiguës, rigides, glabres smr la face interne, portant une toulle de poils sur le dos. Périanthe atteignant lə $1 \mathrm{~mm}$. de long, très villeux, à poils étalés-dressés, tombant en une seule fois après l'anthèse, lobes du tiers de la longueur totale dı périanthe, épaissis au sommet. Étamines à filet isse\% court, aplati, à anthère linéaires ovaire conique, densément velu, à poils dressés, surmonté d'un style grêle, cilié dans sa partie inférieure, s'élargissant en còne, tri- ou quadrangulaire vers le sommet, puis brusquement tronqué en une sorte de cupule d'où sort le stigmate allongé, glabre. Fruit aplati à bords subailés, glabre sauf à la hase qui porte une courte touffe de poils.

\section{Hab. - District Swan (Pritzel, Fraser et Drummond).}

Observations. - Le P. propinqua appartient à la section Terostele caractérisée, ainsi que nous l'avons dit plus haut en examinant le genre et ses subdivisions, par' ses feuilles plus ou moins aplaties, ses cônes axillaires, son périanthe à lobes r'estant soudés sur la plus grande partie de leur longueur', par son style épaissi ver's le sommet et tronqué brusquement sous le stigmate grêle et glabre.

Cette section comprend les espèces suivantes, toutes de la W'estAustrilia :
P. heterophylla Lindl.
P. biloba R. Br.
$P$. propinqua $\mathrm{R}$. Br.
P. squamata R. Br.
$P$. colorata Meisn.
P. stricta $\mathrm{R}$. Br.

Les deux dernières de ces espèces se caractérisent par leur fruit plus densément velu et par l'extrémité du style courtement turbinée sous le stigmate. Dans les quatre autres espèces la villosité n'existe qu'à la base du fruit; bien que ce caractère ne puisse ètre bien observé qu'à 
maturité, on le soupronne déjà dans certaines de nos figures (fig. 24, pl. XXVI).

Le $P$. heterophylla se différencie du $P$. biloba, propinqua et squamata par ses feuilles simples et trifides. La distinction de ces trois dernières espèces est plus difficilc à établir et certains auteurs sont même partisans de les confondre (cf. Diels et Pritzel Fragm. Phyt. Austral. occid., p. 132, in obs. ad. P. biloba R. Br.).

Parmi les notes différentielles proposées par Bentham dans sa Flora Australiensis citons :

Segments des feuilles larges et courts, rarement linéaires dans les feuilles inférieures. Écailles villeuses. Périanthe de 16 à $20 \mathrm{~mm}$. de long . . . . . . . . P.propinqua. Segments des feuilles linéaires. Écailles villeuses. Périanthe de 8 à $10 \mathrm{~mm}$. de long . . P. biloba Segments des feuilles linéaires. Écailles glabres. Périanthe de 8 à $10 \mathrm{~mm}$. de long . . . P. squamata.

Si nous comparons à ces diagnoses les figures de notre planche, nous voyons qu'il est facile d'écarter le $P$. squamata dont les écailles sont glabres, tandis qu'elles sont nettement velues dans notre plante (fig. 19). Mais il est plus difficile de décider entre les deux autres espèces; en effet, pour la longueur du périanthe, la plante que nous avons sous les yeux est intermédiaire entre les deux types ayant moins de 16 et plus de $10 \mathrm{~mm}$. de long. Quant aux caractères tirés des feuilles, ils nous feraient plutôt rapprocher la plante figurée du $P$. biloba, puisque sur la même plante nous trouvons, comme lc montrent les figures 16 et 17 , des feuilles à segments linéaires et élargis.

Le $P$. propinqua figurée sur la planche ci-jointe a été récolté dans le district de la Swan River, dans les forêts ombragées des inontagnes du Darling Range (Plantee Australice occidentalis, n $\left.{ }^{\circ} 592\right)$. 

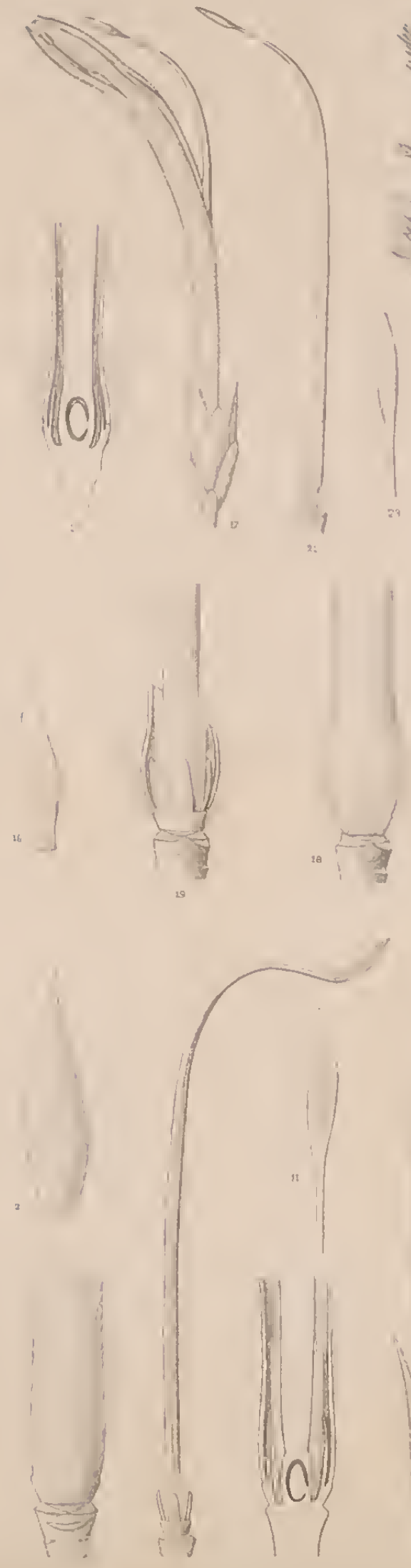

A diApreval ad nat del

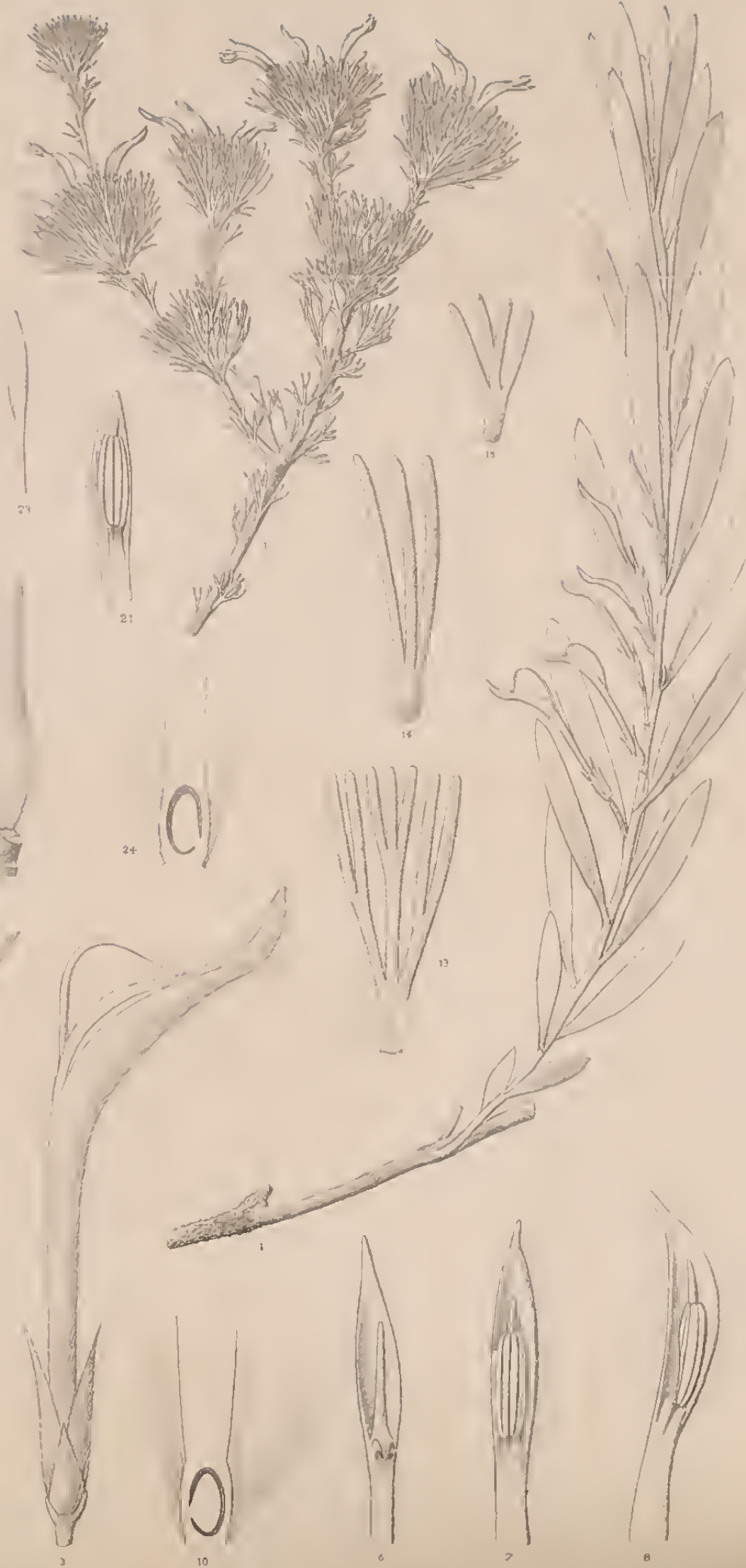

Elablrs Mnol Paris 


\section{ADENANTHOS Labill.}

Le genre Adenanthos, créé par Labillardière, est surtout répandu el Australie occidentale; deux espèces existent également dans l'Australie méridionale, et l'une s'avance inème jusque dans le Victoria; d'aplès les r'echerches r'écentes de MM. Diels ct F'ritzel, d'autres espèces se rencontrent jusque dans les parties occidentales de l'Australie orientale.

Ce sont surtout les régions côtic̀res du West Australia, les districts liyre et Stirling, qui sont riches en représentants de ce genre; ils possèdent une douzaine d'espèccs; dans les régions forestic̀res occidentales elles deviennent moins nombreuses.

L.e genre Adenanthos possède un port très variable : de la plante basse, couchée, à la forme arbor'escente, tel l'A. sericea qui donne dans ces conditions une note particulière dans le paysage. Les feuilles sont également très variables dans leur forme et leur indument. Les fleurs variant du vert au rouge, axillaires ou terminales, sont entourées de bractées imbriquées dont les internes dépassent les externes. C'est ce caractère qui, ainsi que nous l'avons dit plus haut, établit le vrai caractère lu genre. $\Lambda$ côté de ce détail il faut encore citer la forme du tube qui se fend sur la face dorsale pour laisser passer le style et qui se létache après l'antlıc̀sc, laissant à la base un anneau persistant. L'ovaire est entouré de 4 écailles plus ou moins développées.

Les espèces qui composent ce genre sont plus nombreuses que ne l'avait indiqué Bentham dans la Flore australienne, où quatorze espèces sont relevées par lui dans deux sections qui peuvent être encore admises malgré lcs nouveautés décrites depuis la publication de son mémorable travail.

Ces deux sections se caractérisent comme suit et renferment actuellement les espèces suivantes, dont nous indiquons la distribution :

Section Eurylaema. - Tube du périanthe dilaté obliquement et recourbé au-dessus du milieu. Anthère postérieure linéaire, stérile. Feuilles planes, fleur's axillaires.
1. - A. barbigera Lindl. - Australie occidentale.
2. - A. obovata Labill.
3. - A. Detmoldi F. v. Muell. . 
Section Stenolaema. - Tube du perianthe presque droit, non élargi; anthères toutes fertiles.

Sous-section. - Axillares. - Fleurs axillaires.

4. - A. aureola Labill. Australie occidentale.

5. - A. Cunninghamii Meisn.

6. - A. pungens Meisn.

Sous-section. - Terminaies. - Fleurs terminales.

a) Integrifolice.

7. - A. venosa Meisn. Australie occidentale.

8. - A. Dobsoni F. v. Muell.

9. - A. Forrestii F. v. Muell.

10. - A. linearis Meisn.

b) Laciniatee.

11. - A. sericea Labill.

12. - A. cygnorum Diels.

13. - A. Meisneri Lehm.

14. - A. filifolia Benth.

15. -- A. Drummondii Meisn.

16. - A. terminalis R. Br.

et australe.

17. - A. flavidiflora F. v. Muell.

Australie occidentale.

18. - A. argyrea Diels

19. - A. apiculata $\mathrm{R}$. Br.

Dans les notes suivantes relatives à la planche XXVII nous pouvons étudier une espèce de chacune des deux sections; jusqu'à ce jour, de tout le genre Adenanthos, le seul A.terminalis avait été figuré dans l'Iconographie d'Endlicher' sous la planche 110.

\section{Adenanthos barbigera Lindl.}

Swan River App. (1840) p. 36; Meisn. in Pl. Preiss. I (1814) p. 560 et in $D C$. Prod, regn. veget., XIV $\% .311$.

Arbrisseau à tiges simples, dressées, de 30 à $60 \mathrm{~cm}$. de haut, plus ou moins ramifiées et atteignant $1 \mathrm{~m}$. 25 de haut, munies à l'état jeune de poils assez longs, soyeux. Feuilles lancéolées ou elliptiques-oblongues, de $2 \mathrm{~cm}$. i $75 \mathrm{~cm}$. de long, subobtuses, apiculées au sommet, rétrécies à la base en un court pétiolule; nervure médiane et 
une latérale, longitudinales asse\% nettement proéminentes sur la face inférieure. Fleurs solitaires à l'aisselle de feuilles, à pédicelle grêle de 2 à $7 \mathrm{~mm}$. de long. Bractées lancéolés, aiguës, villeuses sur la face externe, les extérieures plus courtes que les intérieures. Périanthe de $2,5 \mathrm{~cm}$. environ de long, densément velu, à poils soyenx, recourbé vers le milieu, à lobes relativement courts, restant soudés pendant assez longtemps, les segments supérieurs se séparant tardivement sur un tiers environ de la longueur du périanthe. chacun d'eux renfermant une étamine à deux loges terminée par le connectif proéminent; segments latéraux légèrement falciformes; segment postérieur un peu plus petit que l'antérieur, à étamine réduite au connectif. Ovaire ovoïde-globuleux, densément velu; style épais cylindrique, recourbé vers le sommet, éparsement velu, à poils dressés, à stigmate elliptique, aplati, strié longitudinalement.

\footnotetext{
Hab. - District Swan (Pritzel et autres); Gordon et Harvey Rivers (Oldfield).
}

Observations. - L'A. barbigera appartient à la section Eurylcema, dont nous avons donné plus haut les caractères; en effet, comme le fait voir nettement la figure 3 de la planche XXVII le tube est obliquement

\section{Explication des figures 1-11 de la planche XXVII.}

\section{Adenanthos barbigera Lindl.}

Fio. 1, - Rameau fleuri (1/1).

Fia. 2. - Bractée florale vue de dos (6/1).

Frc. 3. - Fleur (6/1).

F1G. 4. - Base de la fleur, bractée calicinale enlevée (12/1).

Fic. 5. - Coupe longitudinale de cette base (12/1).

Fro. 6. - Lobe supérieur du périanthe vu de face (8/1).

F1G. 7. - Lobe inférieur du périanthe vu de face $(8 / 1)$.

FiG. 8. - Lobe latéral (8/1).

Fı. 9. - Ovaire entouré de la cupule, surmonté du style, terminé par le stigmate $(4 / 1)$.

Fı. 10. - Coupe longitudinale de l'ovaire (20/1).

FIG. 11. - Stigmate (8/1). 
dilate et recourbé, une anthère est avortée (fig. 6) et les fleurs sont axillaires et solitaires (fig 1).

Comme nous l'avons indiqué également il existe trois espèces dans ce groupe, dont l'A. Detmoldi est la plus récente, créée par F. v. Muell. dans ses Fragmentee Phyt. Australice VIII (1874) p. 149.

Notıe plante se caractérise surtout par ses feuilles elliptiques, oblongues et lancéolées, mesurant de 1,8 à $5 \mathrm{~cm}$. de long, elles sont plus petites chez les deux autres espèces.

La plante qui a servi à faire les analyses des figures 1 à 11 , planche XXVJI, a été récoltée par M. Pritzel dans le Narling Range, en décembre 1900; elle a été distribuée sous le n. 74 dans les Plantæe australia occidentalis.

\section{Adenanthos Meisneri Lehm.}

Pl. Preiss. I (1844) p. 512 et II p. 248; Meisn. in DC. Prod, regn reget. XIV p. 312; Benth. Fl. Austral. V p. 254.

Plante buissonnante, à rameaux plus ou moins rampants ou irrégulièrement étalés, atteignant 1,25 m. de haut; rameaux plus ou moins fortement pubescents. Feuilles plus ou moins velues, une ou plusieurs fois bi- ou trifides, atteionant an maximum $20 \mathrm{~mm}$. de long, à segments cylindriques, subohtus au sommet. Inflorescence ter-

\section{Explication des figures $12-24$ de la planche XXVII.}

\section{Adenanthos Meissneri Lehm.}

Fiv. 12. - Rameau fleuri (1/1).

Fır, 13.15 - Feuilles différemment divisces (4/1).

Fig. 16. - Bractée Aorale (6/1).

Fia. 17 - Fleur épanouie (4/1).

FIG. 18. - Base du périanthe (12/1).

F1o 19. - Base du périanthe dont une partie a été enlevée pour laisser voir la cupule entourant l'ovaire (12/1).

F1G. 20. - Coupe longitudinale de la base du périantlie (10/1).

FIG. 21. - Lobe du périanthe avec son étamine, vu de face (9/1).

F1G. 22. - Ovaire, style et stigmate (4/1).

F1G. 23. - Stigmate (10/1).

Fig. 24. - Coupe longitudinale de l'ovaire (15/1). 
minale formce de 2 a 1 fleurs, Inactées hasilatires ovales aignuës, glabres oi très courtemont ciliées sur le bord, les externes plus courtes que les internes. Périanthe de 18 i $25 \mathrm{~mm}$. de long., legriement recourbé, glanduleux-pubescent, à poils courts nou soyeux, ì lohes subégaux, airus, abritant tous une étamine parfaite i anthère apiculée. ()vare densément velu, entouré a la base par un disque discontinu constitué par 4 lames. Style épais, muni d'un sillon longitudinal, recourbé vers le sommet, glabre dans toute sa longueur, terminé par un stigmate elliptipue, iplati.

Hab. - District Wellington (Pritzel): Pointe d'Entrecasteaux (Wa)cott); Cap Leschenault et environs de Bunbury (Oldfleld).

Olservations. - L'A. Meisneri applartient bicn, comme le montre la liste des espèces du genlre domnée plus haut, à la section Stenolatma. En effet, la figure 17, que l'on peut comparer à la figure 3, montre un périanthe presque droit, non renflé au delà du milieu. Mais la différenciation des espèces dc ccttc scction est plus conpliquéc; en effet, conme le fait voir l'énumération les espèces connues dans le genre, seize types ont été décrits. ('es seize espèces pourraient se répartir dans deux grands groupes, les nos 4 à 6 sc rangeant dans une sous-section A xillares qui, ainsi que le nom que nous proposons l'indique, possèdent des fleurs axillaires et toutes les autres appartenant i une sous-section Terminales, dont les fleur's sont terminales, ce qui d'ailleurs est le cas pour la plante que nous figurons. Dans ce dernier groupe nous pourrions encore établir les subdivisions Integrifolice et Laciniata en nous basant sur les caractères admis par Bentham dans la clef analytique dc son genre et qui sont : feuilles entières et feuilles divisées. Parmi les Integrifolice figurent les $n^{\circ \mathrm{s}} 7$ à 10 ; les autres numéros se rapportent tous aux Laciniata, dont fait partie l'A. Meisneri, figuré pl. XXVII.

Bentham se base ensuite pour différencier' les espèces qu'il adınettait dans ce gemre sur la villosité des segments du périanthc : ceux-ci seraient densément velus sur la face interne chez certaines espèces, glabres chez d'autres; malheureusement ce caractère ne parait pas ctre absolu, puisque l'A. Meisneri est classé par Bentham dans les deux subdivisions pouvant posséder un perianthe glabre ou velu sur la face interne. De cettc manière si la plante se présente avec un périanthe velu, ce qui n'est pas le cas pour le sujet que nous figurons, elle paraît voisine de l'A. sericea, dont le périanthe est velu-soyeux, tandis qu'il est éparsement 
velu-glanduleux che\% l'A. Meisneri. Il serait done égaloment voisin de l'A. cygnorum Diels (in Diels et Pritzel Fragm. Phyt. Austral. occid., p. 138), qui serait l'A. apiculatı Meissn.; en rétablissant cette espèce M. Diels n'en a pas d'ailleurs fixé les caractères différentiels. Si par contre l'A. Meisneri se présente avec un tube du périanthe glabre, il se différenciera par la longueur du périanthe atteignant $2,5 \mathrm{~cm}$. de long et par la présence de poils glanduleux sur la face extérieure du périanthe.

Nous tenons encore à ajouter que le caractère tiré de la glabréité interne du tube du périanthe parait de valeur secondaire : car l' $A$. argyrea Diels, que nous sommes arrivé à classer dons le voisinage de $A$. flavidifora d'après les données de l'auteur lui-même, possède, d'après la description qui en a été publiée, des lobes velus sur la face interne, sous les anthères; ce caractère devrait done faire classel cette plante dans le voisinage de l' $A$. filifolia en tenant compte pour ce rapprochement de la longueur des fleurs que nous n'avons pu étudier, pas plus que celles de l'A. argyrea. 
Pl Novae Herb. Hort. Thenensis.

PL.XXIX

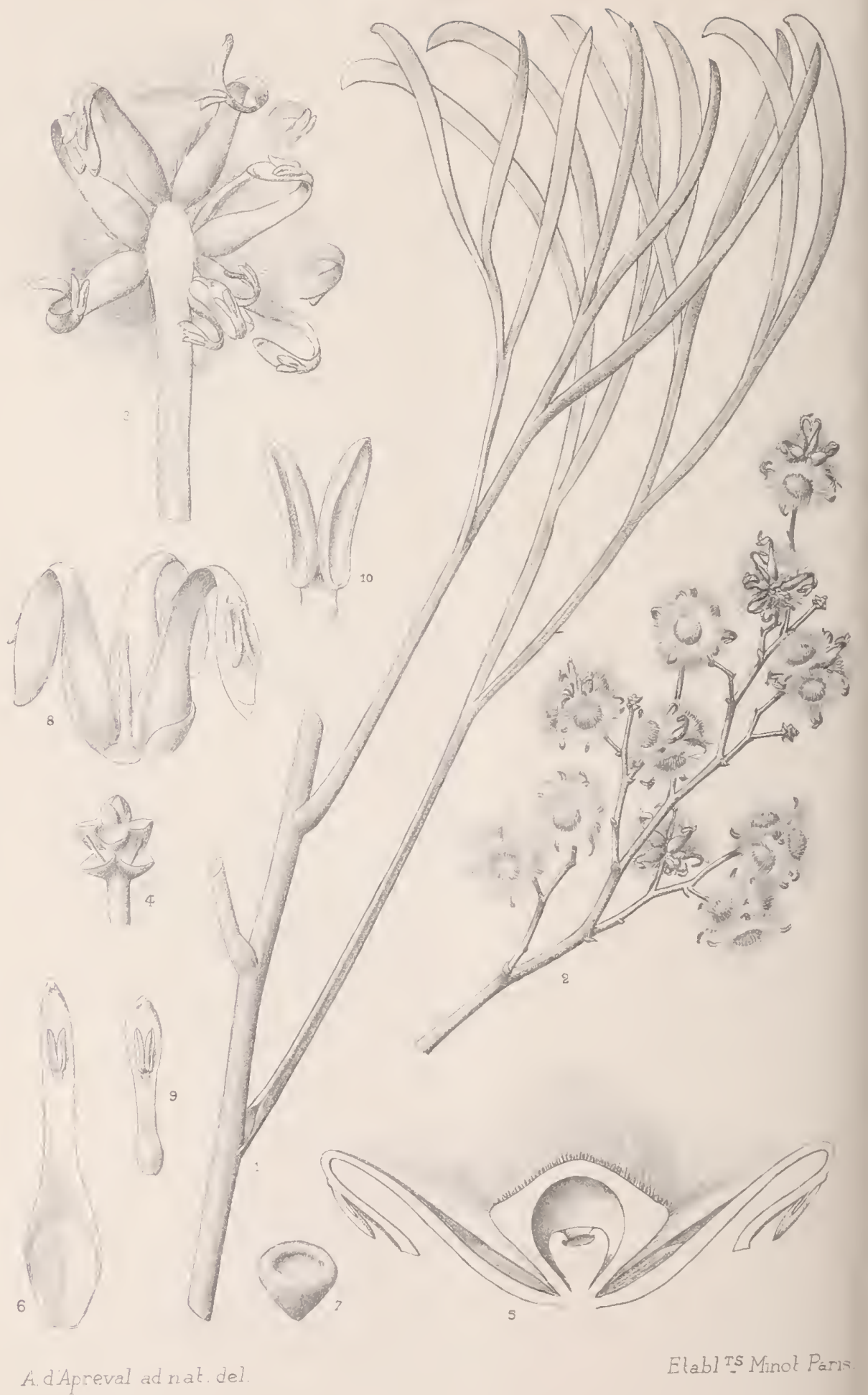

SIMSIA LATIFOLIA R. Br. 



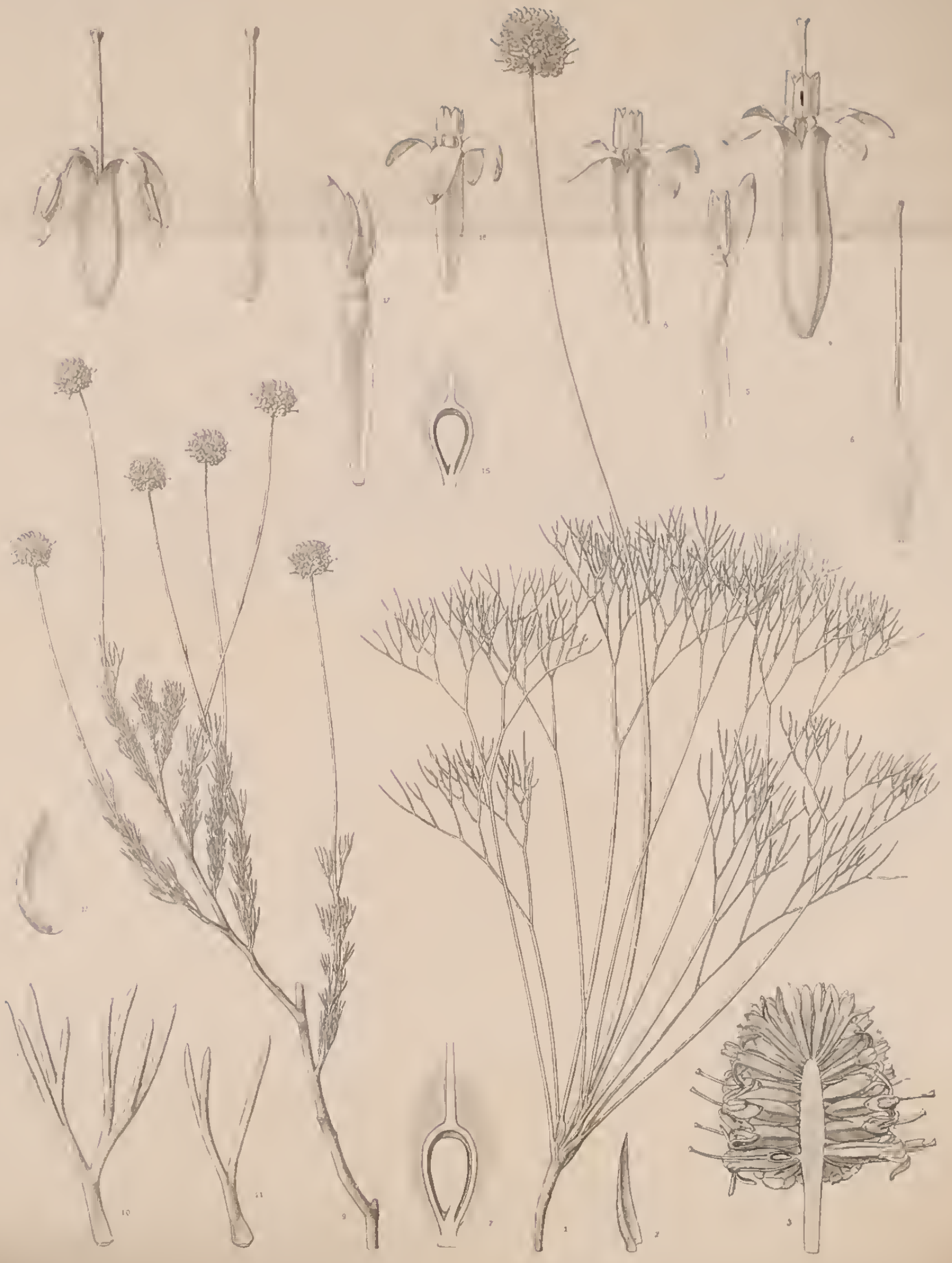




\section{SIMSIA R. Br.}

Ce genre a été créé en 1810 par' R. Brown dans les Transactions de la Société Linnéenne de Londres. C'est sous le nom générique de Stirlingia que les espèces de ce genre se trouvent signalées dans la Flor'e d'Auslie de Bentham; mais ce nom, proposé par Endlicher en 1838 seulement, ne peut avoir la priorité sur celui de $\mathrm{R}$. Brown qui est de vingt ans plus vieux que lui. La remise en honneur de l'appellation première a été faite par M. le prof. Ad. Engler' dans la révision de la famille des Proteacece publiée dans les Pflanzenfamilien et MM. Dalla Torre et Harms l'ont également admise dans leur Genera Siphonogamarum.

Le genre Simsia est endémique dans l'Australie occidentale et localisé surtout dans la partie sud-occidentale, les espèces relativement peu nombreuses qui le constituent se rencontrant surtout dans les forêts légères à sol silicieux ou sablonneux. Par leur port, les Simsia rappellent plus ou moins les Composées et constituent par leur fréquence et leur associations des plantes caractéristiques des terrains pauvres et sablonneux.

Le genre Simsia ne renferme que cinq espèces, qui sont énumérées dans la Flore d'Australie de Bentham sous le nom de Stirlingia. De ces espèces, les $S$. tenuifolia $R$. Br. et $S$. latifolia $R$. Br., ont été créées sous le nom générique de Simsia, les autres sous celui de Stirlingia et c'est le baron F. von Mueller qui en a fait la transposition en 188? dans son Census des plantes d'Australie

Les Simsia constituent des arbrisseaux ou des sous-arbrisseaux généralement glabres, à feuilles divisées et variables de forme. Les inflorescences sont portées par des pédoncules terminaux assez longs et privés de feuilles, simples ou ramifiées. Les fleurs réunies en épis globuleux ou en glomérules à l'extrémité des hampes ou de leur's ramifications sont généralement hermaphrodites à la base des inflorescences, mâles par avortement ver's le sommet, leur périanthe est régulier à 4 lobes étalés lors de l'anthèse. Les anthères sont toutes fertiles. Le style dépasse en général les étamines et l'ovaire; généralement velu, renfer'me un seul ovule dressé.

Nous avons pu faire dessiner des analyses des $S$. simplex F. v. Muell., 
S. abrotanoides F. v. Muell. et $S$. latifolia $\mathrm{R}$. Br. qui n'ont pas encore été figurées jusqu'à ce jour; seul du genre, le S. tenuifolia Endl. avait jusqu'à présent été représenté dans l'Iconographie des Protéacées de cet auteur'.

\section{Simsia simplex F. v. Muell.}

Census of Austral. pl. (1882) p. 66.

Stirlingia simplex Lindl. Swan River App. (1840) p. 30; Meisn. Pl. P'reiss. I p. 516 et in DC. Prod. regn. reget. XIV p. 326 ; Benth. Fl. Austral Y p. 357.

Stirlingia capillifolia Meisn. in Hook. Kew Journ. VII (1855) p. 70 et in $D C$ loc cit.

Petite plante herbacée à tige feuillue courte. Feuilles plusieurs fois di-ou trichotomes, atteignant $15 \mathrm{~cm}$. de long, à segments subaigus au sommet, orêles, assez rigides à l'état adulte, à nétiole dressé plus ou moins engaînant à la base. Inflorescence partant du centre de la touffe de feuilles qu'elle disperse, simple ou parfois ramifiée à la base, atteignant $45 \mathrm{~cm}$. lle long et portant un seul épi au sommet. Fleur's densément réunies formant un capitule de 15 à $18 \mathrm{~mm}$. de diamètre, munies chacune d'une bractée plus courte que le périanthe, lancéolée, aiguëacuminée. Périanthe des fleurs inférieures hermaphrodites

Explication des figures 1-8 de la planche XXVIII.

Simsia simplex $v$. Muell.

Fir. 1. - Plante lleurie (1/1).

Fir, 2, - Baclée forale (10)1)

len, 3. - Coupe longiludinale du capilule floral (21/2/1).

Fıg. 4. - Fleur hermaphrodite épanouie ( $/ 1$ ).

Fig. 5. - Un des lobes du périanthe de cette flour $(8 / 1)$.

Fig. 6. - Ovaire, style el stigmale (7/1).

Fıa. 7. - Coupe longitudinale de lovaice $(10 / 1)$.

Fig. S. - Fleur mile épanouie (T/1) 
de $8 \mathrm{~mm}$. environ de long, à lobes réfléchis pendant l'anthèse, épaissis et subaigus au sommet. Étamines se détachant du périanthe au niveau de la gorge du tube, à filet aplati, à loges parallèles, séparées à la base et au sommet, conniventes formant un anneau autour du style. Ovaire obovoïlle, densément velu, à ovule solitaire, dressé; style environ deux fois aussi long que l'ovaire, dépassant le tube staminal, à stigmate légèrement élaroci au sommet. lileurs supérieures mâles, à périanthe un peu plus réduit, ¿ ovaire avorté.

\section{Hab - Distrlct Swan (Pritzel): entre les rivières Moore et Mur- chison (Drummond).}

Observations. - Le $S$. simplex est caractérisé entre autres par ses feuilles, qui, comme le montre la figure 1 de la planche XXVIII, sont divisées en lobes cylindriques, rigides. Ce caractère est commun dans ses traits généraux aux quatre premières espèces du genre; les $S$. simplex, S. abrotanoides F. v. Muell., S. teretifolia F. v. Muell. et S. tenuifolia $\mathrm{R}$. Br.

Pour différencier ces quatre espèces, on peut se baser sur la forme et la grandeur des bractées florales : tandis que chez les deux premières qui nous préoccupent, ces bractées sont relativement étroites variant de longueur de la moitié du périanthe à sa longueur totale, chez le S. teretifolir elles sont larges, ciliées, imbriquées dans l'inflorescence spiciforme jeune et aussi longues que le périanthe; chez lc $S$. tenuifolia par contre elles sont très réduites.

Parmi les caractèr's communs des deux premières espèces il faut encore citer la for'me de l'infloresceuce qui est généralement un capitule solitaire à l'extrémité du pédoncule allongé, rarement bi- ou trifurquée, clacune des ramifications étant terminée par un capitule. Mais tandis que chez le $S$. simplex le pédoncule de l'inflorescence peut atteindre de 30 à $45 \mathrm{~cm}$. de long, il ne dépasse guère $10 \mathrm{~cm}$. chez le $S$. abrotanoides. Un coup d'œil jeté sur les figures de notre planche XXVIII fera, mieux qun toute description, saisir les caractères diflérentiels de l'espèce.

La plante qui a servi à faire les dessins de la planche ci-jointe, a été insérée par ..H. Pritzel dans ses Planta Australice occidentalis sous le $n^{\circ} 718$, et provient du district Swan, dans le Darling Range, septembre 1901 . 


\section{Simsia abrotanoides F. v. Muell.}

Census of Austral. pl. (1882) p. 66.

Stirlingia abrotanoides Meisn. in Pl. Preiss. I (1844) p. 517 en in DC. Prod. regn. veget. XIV p. 326; Bentham Fl. Austral. V p. 357.

Petite plante sous-buissonnante, à tiges sous ligneuses, assez grêles, simples ou ramifiées, atteignant environ $30 \mathrm{~cm}$. de hauteur. Feuilles petites, de 10 à $25 \mathrm{~mm}$. de long bi- ou trifurquées, ou di- ou trichotomes, à pétiole plus court que le limbe dont les segments sont cylindriques, grêles, dressés, subaigus. Inflorescence terminant les rameaux, à pédoncule grêle atteignant environ $7 \mathrm{~cm}$. de long et muni souvent d'une bractée, feuille avortée vers le milieu de sa longueur. Capitule floral terminal, solitaire, à fleurs garanties par une bractée ovale-aiguë, concave, plus courte que le périantlie, Fleurs basilaires hermaphrodites, à périanthe féndu jusque vers le milieu en 4 lobes subaigus, épaissis au sommet, réfléchis pendant l'anthèse à étamine à deux loges parallèles. Ovaire obovoïde, densément velu, à ovule dressé ; style au moins deux fois aussi long que l'ovaire, à stigmate légèrement élargi au sommet. Fleur du sommet du capitule mâles, à périanthe non renflé à la base, à ovaire avorté.

Hab. - District Avon (Pritzel); Swan Hiver (Drummond et Preiss): Cabinyong (Oldfield).

Explication des figures 6-17 de la planche XXVIII.

Simsia abrotanoides $v$. Muell.

FiG. 9 - Fragment de la plante fleurie (1/1).

FIG. 10-11. - Deux formes de feuilles (6/1).

FIG. 12. - Bractée florale (20/1).

Fro. 13. - Fleur hermaphrodite (10/1).

Fic. 14. - Ovaire, style et stigmate (10/1).

Fro. 15. - Coupe longitudinale de l'ovaire (18/1).

FIG. 16. - Fleur màle (10/1).

FIG. 17. - Lobe du périanthe de la fleur mâle (14/1). 
Observations. - I.e S. abrotanoides est, comme nous l'avons signalé à propos de l'espéce précédente, caractérisé par scs fcuilles à lobes filiformes, rigides, ses bractées relativement petites ct ses infloresecnees formées de capitules isolés terminant un long pédoncule.

Les caractères différentiels de cette espèce et de la précédente ont été déjà indiqués plus haut, ils résident dans la grandeur des fouilles et dans celle des pédoncules des inflorescences. Un coup d'œil jeté sur les figures comparatives des éléments de ces deux plantes figurés planche XXVIII, fera facilement saisir les différences de ces deux plantes qui se ressemblent uniquement par leurs fleurs.

Ia plante figurée a été recueillic par $\mathrm{M}$. le $\mathrm{I}^{\mathrm{r}^{\mathrm{r}}}$ Pritzel dans le district Avon, en octobre 1901, dans la brousse; elle porte le $n^{\circ} 760$ de ses Plantoe Australice occidentalis.

\section{Simsia latifolia $R . B r$.}

Prot. Nov. (1889) p. 9 in Trans. Linn. Soc. X (1810) p. 152.

Stirlingia latifolia Steud. Nom. Bot. ed. 2, II (1840) p. 644; Meisn. in Pl. Preiss I p. 517 et in $D C$. Prod. regn. veget. XIV p. 325 ; Bentham Fl. Austral. V p. 358.

Stirlingia paniculata Lindl. Swan River App. (1840) p. 30.

Plante buissonnante; tiges feuillues atteignant rarement $30 \mathrm{~cm}$. de hauteur, simples ou ramifiées. Feuilles une, deux ou trois fois bifides ou trifides, à pétiole canaliculé et embrassant à la base, à segments rigides, linéaires ou

\section{Explication des figures de la planche XXIX.}

Simsia latifolia $R . B r$.

FIG. 1. - Fragment de la tige avec feuilles (1/1).

Fig. 2. - Fragment de l'inflorescence (1/1).

Fig. 3. - Coupe longitudinale d'un capitule floral (3/1).

FıG. 4. - Rachis du capitule après enlèvement des fleurs (3/1).

Fig. 5. - Fleur hermaphrodite en coupe longitudinale (5/1).

FIF. 6. - Lobe du périanthe de cette fleur (4/1).

Fig. 7. - Ovule (6/1).

Fıa. 8. - Fleur mâle, un sépale enlevé pour laisser voir le style avorté (8/1).

Fig. 9. - Lobe du périanthe de la fleur male (4/1).

Fig. 10. - Étamine vue de face (14/1). 
étroitement lancéolés, atteignant $10 \mathrm{~cm}$. de long, parfois recourbés au sommet, et jusqu'à $2,5 \mathrm{~cm}$. de diamètre dans certains échantillons de la Swan River. Inflorescence pouvant atteindre $45 \mathrm{~cm}$. de long, constituant une panicule ramifiée, à rameaux munis à leur base de petites bractées aiguës. Capitules ou épillets portés à l'extrémité de pédicelles de 2 à $6 \mathrm{~mm}$. de long, formés de 5-7 fleurs, protégées chacune par une bractée petite, cupulaire, subarrondie au sommet. Périanthe des fleurs mâles constitué par 4 lohes subspatulés portant dans la partie supérieure l'étamine à deux loges légèrement divergentes vers le sommet, ovaire avorté remplacé par un stylet. Fleurs femelles à 4 lobes élargis à la base et rétrécis vers le sommet qui porte une étamine à deux loges légèrement avortées. Ovaire turbiné, densément velu-hérissé, contenant un ovule dressé.

Hab. - District Swan (Pritzel); du Kivg George's Sound jusqu'à la Swan River (divers): dans la région de la Murchison River (Oldfield).

Observations. - Le Simsia latifolia diffère notablement des deux espèces dont nous avons donné des figures planche XXVIII : en effet, au lieu de posséder des feuilles à segments cylindriques, filiformes, rigides, les feuilles également divisées sont à segments aplatis, linéaires ou lancéolés. Tandis que chez les autres espèces du genre, dont nous avons donné l'énumération plus haut, les inflor'escences sont constituées par des capitules solitaires à l'extrémité d'un pétiole peu ou pas divisé, chez cette espèce l'inflor'escence est paniculée, assez fortement ramifiée et les fleur's sont réunies à l'extrémité de courts pédicelles comme le montre la figure 2 de notre planche XXIX.

De ce qui précède, il résulte que nous n'avons pas à insister davantage sur ces caractères suffisamment mis en évidence par les analyses de la planche.

La plante figurée provient du Swan River district; elle a été recueillie en novembre 1900 et porte le $n^{\circ} 28$ dans la collection des Plantce Australiw occidentalis. 


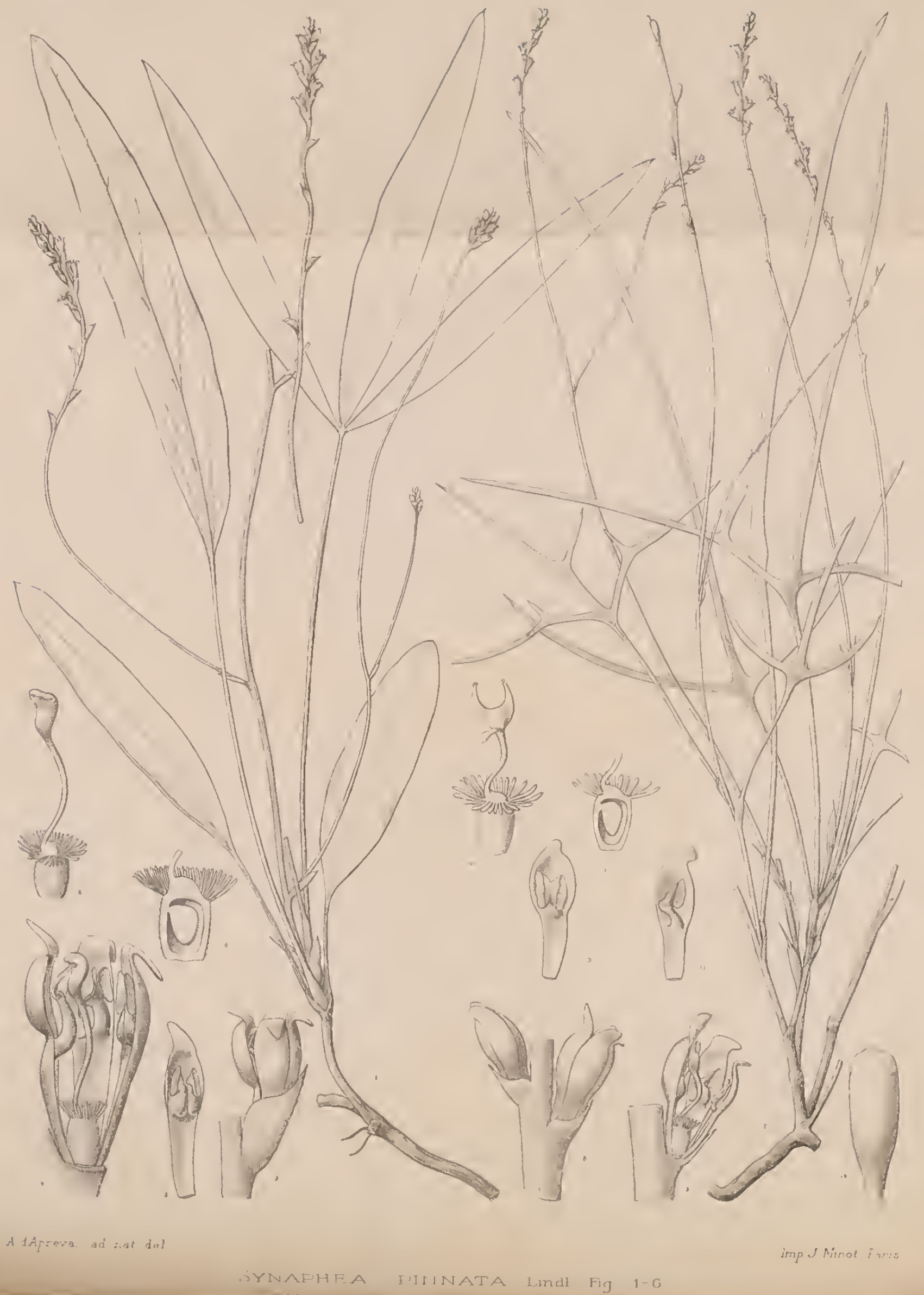




\section{SYNAPHEA R. Br $r$.}

l.c genre Synaph'a créé par R. Brown, occupe par scs caractères spéciaux une place assez particulière dans lo groupement des Protéacées Les huit espèces qui le constituent sont toutes endémiques dans la West Australia et se retrouvent surtout dans la partie sud-ouest du pays. Elles possèdent comme caractères communs la forme de leurs inflorescences simples ou ramifiées, denses au début, généralement allongées, formées de fleurs petites sessiles, garanlics par une bractée concave. C'es fleur's sont hermaphrodites, à périanthe oblique ou légèrement recourbé, à tubc court, à lobes libres jusqu'à la hase. Les étamines sont caractéristiques, celle qui sc trouve opposée au lobe antérieur du périanthe possédant deux anthc̀'es et les étamines latérales n'en possédant qu'unc; l'étaminc postérieure est réduite au filet qui s'applique sur le stigmate, comme le montrent les figures analytiques de nos planches suivantes. L'ovaire est caractérisé par une couronne de poils glanduleux. plus ou moins en massue; le style est dilaté au sommet où il prend des formes qui varient suivant les espèces.

Nous n'insisterons pas davantagc sur les caractères différentiels de ce genre dont nous avons au début examiné sommairement les affinités.

Les huit espèces de ce genre peuvent se ranger en deux groupes, dont le premier ne renferme qu'une seule espèce, le $S$. polymorpha $\mathrm{R}$. Br., caractérisé par ses épis floraux simples n'excédant pas les feuilles florales, courtement pétiolées; cette espèce est donc à écarter', elle n'a pas do rapports étroits avec les deux plantes de notre planche XXX. Les autres espèces : I. dilatata $\mathrm{R}$. Br., facosa $\mathrm{R}$. Br., Preissii Meisn., acutiloba Meisn., petiolaris R. Br., decorticans Lindl., pinnata Lindl., possèdent au contraire des feuilles longuement pétiolées et des inflorescences allongées, grêles, privées de feuilles et souvent ramifiées.

Nos matériaux nous permettent de figurer ici les $S$. favosa $\mathrm{R}$. Br., et $S$. pinnata Lindl. dont il n'a pas été, jusqu'à ce jour, publié d'analyses. 


\section{Synaphea pinnata Lindl.}

Swan River App. (1840) p. $32 ;$ Meisn. in Pl. Preiss. I p. 530 et in DC.

l'rod. regn. veget. XIV p. 3I6; Benth. Fl. Austral. V p. 362.

Plante à tige feuillée très courte, rampante, glabre ou parfois velue à la base et sur le rachis de l'inflorescence. Feuilles assez longuement pétiolées, très polymorphes, les inférieures ovales-lancéolées, simples, à limbe deux fois aussi long que le pétiole, ou à limbe aussi long que le pétiole, atteignant $12 \mathrm{~cm}$. de long et $2 \mathrm{~cm}$. de large, les autres trifoliolées, à limbe plus court que le pétiole celui-ci atteignant $13 \mathrm{~cm}$. de long, parfois quintifoliolées; segments atteignant $10 \mathrm{~cm}$. de long et tiers ou parfois trilobés, à lobes plus ou moins décurrents. Inflorescences atteignant $30 \mathrm{~cm}$. de long, privées de feuilles, grêles, simples ou ramifiées, munies au niveau des ramifications de petites bractées ovales. Fleur's plus ou moins espacées vers l'extrémité des rameaux, peu nombreuses, alternes. Bractées florales de 2 à $\mathrm{mm}$. de long, ovales-aiguës, courtement velues sur le dos. Périanthe de $6 \mathrm{~mm}$. de long environ, à lohes onguiculés, le postérieur fortement concave, à éta. mine réduite, à filet terminé par une languette, les latéraux à étamine réduite à une loge, l'antérieur à étamine à deux loges portées sur un filet presque divisé en deux.

\section{Explication des figures 1-6 de la planche XXX.}

Synaphea pinnata Lindl.

FIG. 1. - Fragment de la plante (1/1).

Fig. 2. - Fleur avec sa bractèe (5/1).

Fig. 3. - Pétale antérieur vu de face (5/1).

FIG. 4. - Fleurs dont un pétale latéral a été enlevé pour laisser voir la disposition des anthères sur les pétales latéral et posterieur (7/1).

F16. 5. - Ovaire, style et stigmate (7/1).

FIG. 6. - Coupe longitudinale de l'ovaire (10/2). 
Ovaire velu subtronqué au sommet, portant une courome de poils claviformes. Style latéral grêle, glabre, renflé au sommet, mais sans appendice.

\section{Hab. - District Swan (Pritzel).}

Observations. - Le S. pinnata Lindl. applartient comme nous l'avons dit en examinant les caractères généraux du genre, au sccond ct au plus inportant des groupes de Synaphea. La figure 7 donnc bien la caractéristique de ec groupe; mais s'il est facile de déterminer le genre Synaphea, il l'est beaucoup moins de déterminer les espèces affines de cette section. Bentham, dans la Flora australiensis, sc basc, pour séparer spécifiquement les sept espèces que nous avons énumérées plus haut, sur la forme du stigmate et il les classe dans les groupes suivants :

Stigmate à deux cornes.

Stigmate prolongé en un appendice unique, oblong, recourbé, entier ou bilobé.

Stigmate prolongé en un appendicc élargi, court, entier ou bilobé.

Stigmate à deux lobes latéraux élargis ou plus ou moins confluents.

Stigmate élargi, sans lobes ni appendices.

A ces caractères viennent s'ajouter des car'actères accessoir'es tirćs des segments foliaires et sur lesquels nous aurons à revenir.

Si l'on jette un coup d'œil sur les figures 3 et 4 de la planche ci-contre, qui montrent à différents grossissements le style et le stigmate, on verra que la plante dont nous donnons des figures analytiques ne pourra se caser que dans la dernière subdivision; elle s'y trouve en effet toute seulc. Mais Benthan cite dans la clef analıtique du genre parıni les caractères de cette cspèce : Leuf segments long, distinct, ulmost petiolulale. Or. cette description ne correspond pas totalement à ce que l'on pourra voir dans notre figure 7 ; les feuilles de la plante figurées sont à segments plus ou moins allongés, distincts, mais ì pétiolules très courts ou sessiles. Il est vrai que dans ce gemre, comme d'ailleur's dans beaucoup de Protéacées, le système foliairc est très variable et que l'on ne peut guère se baser totalement sur lui pour différencier les espèces; nous pouvons cncore à cc propos faire r'emarquer que les premières feuilles sont r'clativement courtement pétiolées et non divisées en folioles.

Les figures 1-6 de la planche XXX sont exécutées d'après les échantillons recueillis, en septembre 1901, dans le Stirling lRange (Swan Ri- 
ver district) par M. E. Pritzel et portant le $n^{\circ} 719$ dans ses Plantae Australicue occidentalis. 11 est à remarquer qu'en dehors du Swan district cette espèce ne paraît pas encore avoir été rencontrée en Australie.

\section{Synaphea favosa $R . B r$.}

in Trans. Limn. soc. X (1810) p. 156, et Prod. p. 369; Meisn. in Pl. Preiss. Il p. 251 et in DC. Prod. regn, veget. XIV p. 314; Benth. Fl. Austral. V p. 361.

Plante à tiges courtes ou plus ou moins rampantes, glabres ou munies de poils à la base des feuilles ou le long des rachis floraux. Feuilles atteignant $25 \mathrm{~cm}$. de long, longuement pétiolées, à pétiole élargi, engainant à la base, à limbe entier lancéolé-linéaire, ou trilobé, à lobes simples ou bilobés. Inflorescence simple ou ramifiée, longuement pédonculée, parfois munie vers la base de bractées, égalant ou dépassant les feuilles. Fleurs alternes, disposées le long du sommet de l'intlorescence, assez lâches. Bractée florale courte, ovale-aiguë, glabre. Périanthe de 4 à 5 mun. de long, à 4 lobes, le postérieur le plus développé, l'antérieur le plus petit, dans ces deux lobes l'étamine est complète, à deux loges; lobes latéraux à étamines à une loge. Ovaire glabre, ovoïde-tronqué, terminé par une couronne de poils

\section{Explication des figures 1-13 de la planche XXX.}

Synaphea favosa $R . B r$.

Fig. 7. - Fragment de la plante (1/1).

FIg. 8. - Fragment du rachis floral avec bouton et lleur (T/1).

Fıa. 9. - Fleur dont un sépale latéral a été enleve pour laisser voir la dis josition des étamines (7/1).

FIF. 10. - Pétale antérieur à étamine complète ( //1).

Fig. 11. -- Pétale latéral à ètamine à une loge (T/1).

FIG. 12. - Ovaire, style et stigmate (12/1).

F10. 13. - Coupe longitudinale de l'oraire (12/1).

Fra. 14. - Fruit (5/1). 
épais suluclaviformes; style latéral, grèle, à stigmate élargi en demi-lune, la languette de l'étamine postérieure vient s'appliquer contre sa base. Fruit obovoïde, rétréci en un pédicelle un peu plus court que lui.

\section{Hab. - District Murray (Pritzel): King George's Sound et au nord d'Albany (divers).}

Observations. - Le $S$. favnsa, figuré sur la planche XXX, appartient, d'après les indications qui précèdent, à la subdivision du geme Synaphea dont les feuilles sont longuement pétiolées et les inflorescences privées des feuilles, longues et gr'êles.

Comme nous l'avons dit en parlant du S. pinnala, les divisions secondaires du genre sont basées sur la forme du stigmate; celui-ci comme le montre la figure 12 est bien nettement à deux cornes; l'espèce que nous figurons appartient done bien à la première de ces divisions, dans laquelle l existe deux espèces :

S. dilatate R. Br. et $S$. favosa R. $\mathrm{Br}^{\text {r }}$.

Il est assez facile de séparer spécifiquement ces deux plantes; en effet, tandis que chez la première la base du pétiole est hirsute, les feuilles entières ou courtement lobées, chez le $S$. favosa la base du pétiole est comme le reste de la feujlle, les feuilles elles-mêmes sont toutes glabre, sauf les inférieures, profondément lobées. En outre, chez le $S$. dilatata 'inflorescence est pubescente, tandis que chez le $S$. favosa s'il existe des poils, ils sont épars sur le lachis. Tous ces caractères sont présentés dans les figures 7 à 11 de la planche XXX.

Ces figures ont été exécutées d'après une plante recueillie par M. Pritzel, dans les forêts claires d'Eucalyptus marginatus du district Murray (Plantae Australiae occidentalis, n. 115). 



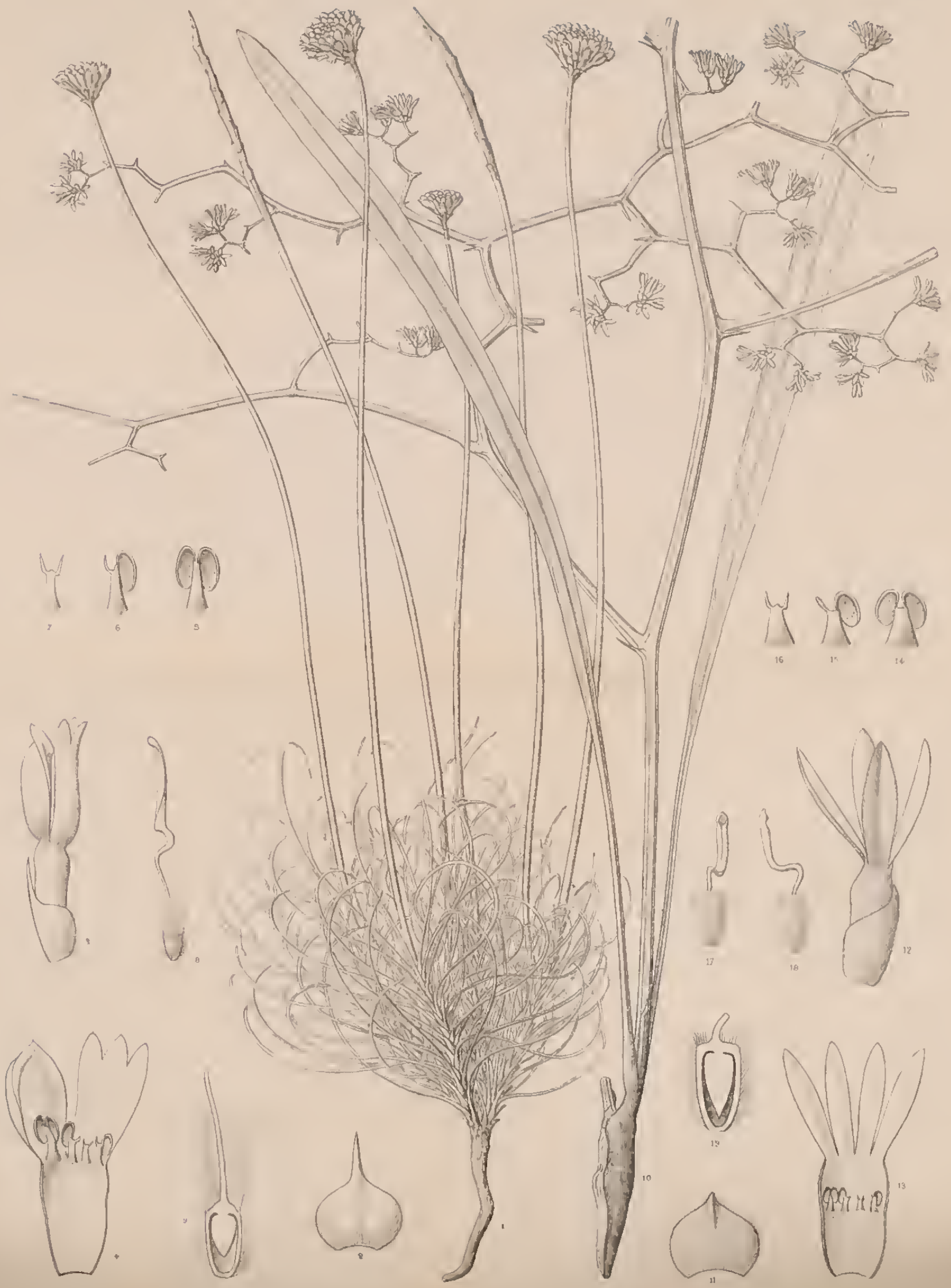


PL Nolue Herb. Hort. Thenensis

PL XXXI

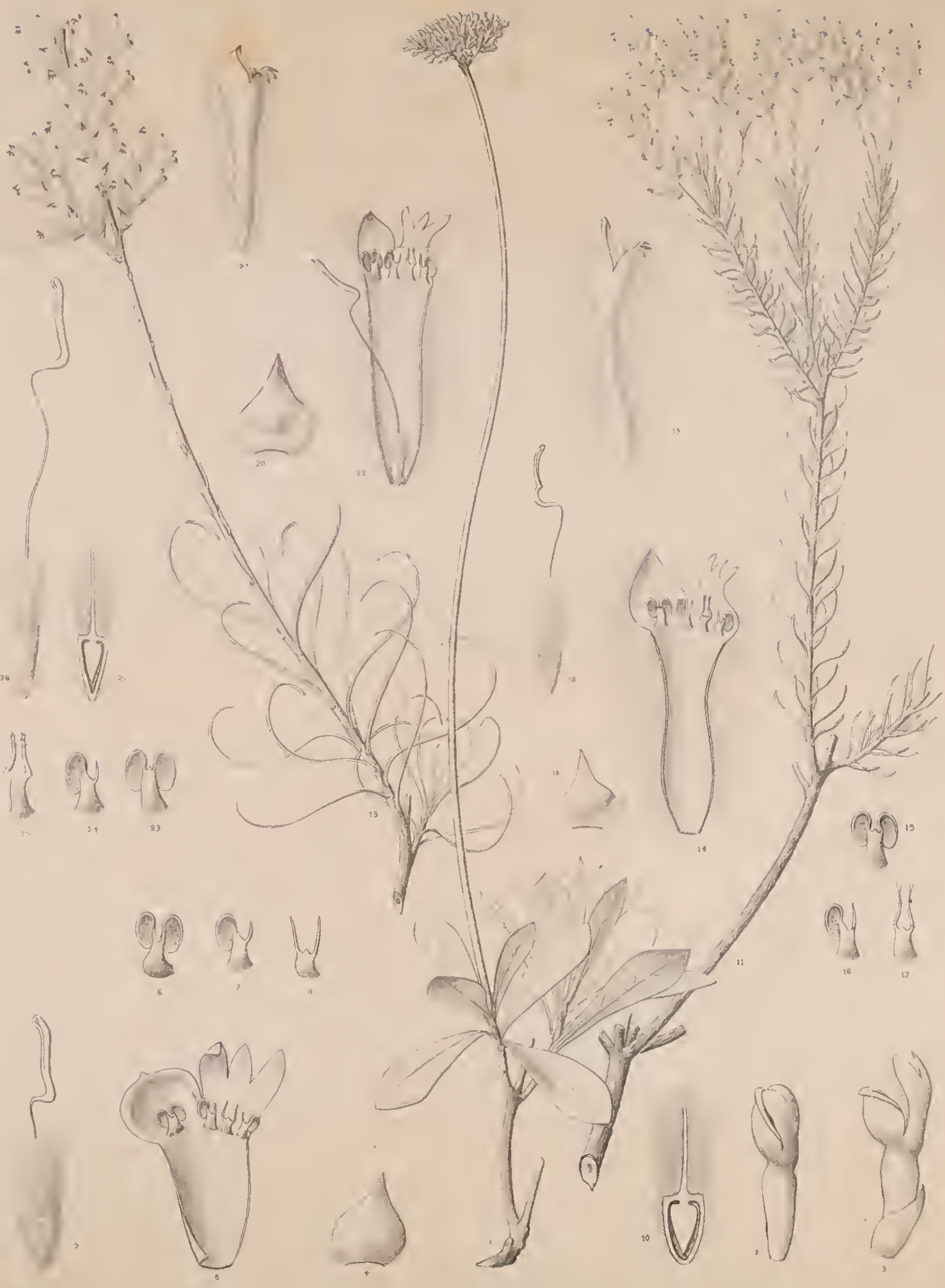




\section{CONOSPERMUM Sm.}

Le genre Conospermum a été créé par Smith en 1798; il renferme une trentaine d'espèces qui, tout ell présentant de nombreux caractères communs, sont relativement faciles à différencier les unes des autres. La plupar't de ces espèces, sont localisées dans la partie extratropicale de l'Australie occidentale.

Les Conospermum se caractérisent par leurs fleurs hermaphrodites, bleuâtres ou blanchâtres, en épis ou solitaires, à tube du périanthe subcylindrique, à limbe bilabié ou à 4 lobes subégaux, et par leurs étamines dont la forme bizarre est très constante pour tout le genre. L'étamine postérieure ou supérieure est toujours complète, c'est-à-dire qu'elle est constituée, comme le font voir les analy'ses des planches des cinq espèces de ce genre que nous étıdions ci-après, par un filet court terminé par deux loges pollinifères non conniventes; l'étamine antérieur'e est réduite au filet. surmonté de deux sortes de stylets plus ou moins développés, parfois divisés et plus ou moins divergents; les étamines latérales sont constituées par un filet à l'extrémité duquel on trouve d'un côté une loge d'étamine, de l'autre un stylet. L'ovaire est obovoïde chez toutes les espèces du genre, plus ou moins velı sur les parois latérales mais toujours couronné par une touffe de poils; le style qui le termine est filiforme, à extrémité stigmatique renflée, sillonnée longitudinalement dans sa partie supérieure. Quant au fruit, il forme une sorte de nucule obconique, surmonté de la touffe de poils qui termine l'ovaire et qui est persistante.

Les Conospermum se présentent sous l'aspect de petits arbrisseaux ou de sous-arbrisseaux. On en a décrit actuellement 35 espèces, qui se lépartissent encore plus ou moins bien dans les deuxs ections qu'avait admises Bentham, c'est-à-dire : Isomerum et Euconospermum. Comme on pouvait le pressentir par les caractères génériques que nous avons sommairement rappelés plus haut, le véritable caractère différentiel de ces deux groupes est celui tiré de la forme du périanthe. qui peut être terminé par 4 lobes subégaux ou par 2 lèvres, l'une d'elles formée par la soudure de 3 lobes. Bentham dans la Flora australiensis donne comme suit, les caractères de ces deux sections : 
Section I. - Isomerum R. B1*. : Lobes du périanthe aussi longs ou plus longs que le tube, subégaux et subétalés, à étamines logées dans le renflement supérieur du tube, un peu plus gibbeux sur le côté dorsal.

Section II. - Euconospermum Endl. : Limbe du pèrianthe bilobé, aussi long ou plus court que le tube, à lèvre supèrieure élargie, concave, lèvre inférieure trilobée.

Cependant MM. Diels et Pritzel, dans leur importante étude basée sur l'observation de la nature, émettent quelques remarques contre cetce classification. D'après les deux auteurs allemands, la section Isomerum devrait ôtre décomposée : les $C$. capitatum R. Br. et petiolare $\mathrm{R} . \mathrm{Br}^{\text {r }}$. constitueraient une section spécialc, celle des Capitatox. Le C. teretifolium R Br. formerait à lui seul une section monotype et quant au C. flexusum $\mathrm{R}$. Br. que $\mathrm{R}$. Browne considérait comme le trpe de la sectiou Isomerum, il y aurait lieu de l'étudier en détail pour juger les caractères qui le rapprochent ou le différencient des autres espèces.

Dans la deuxième section Euconospermum les auteurs allemands considèrent les espèces 5 à 25 de Bentham, soit avec les espèces nouvelles, vingt-cinq espèces énumérées ci-a près, comme formant le noỵan du genre ct les autres espc̀ces 26 à 33 pourraient être réunies en un autre groupe sous le nom de Trichontre; toutes ces espèces sont d'ailleurs trìs affines et par suite très difficiles à classer. MT. Diels et Pritrel sont donc d'aris que la classification proposée par Bentham pnurrait être améliorée et ils sont encore fortifiés dans cette idée par la distribution géographique de ces curieuses et intéressantes plantes. Malheureusement, ils n'ont pas ajouté à ces observations un tableau des caractères sur lesquels ils se basent pour différencier leur's subdivisions nourelles.

La section Euconospermum envisagée dans son sens étroit, est la seule qui se rencontre à la fois dans la région occidentale pt la région orientale dı grand continent australien ; c'est aussi le groupe qui s'étend le plus loin dans le pays, lc C. Toddii F. v M., par exemple, ayant été trouvé trouvé près de Victoria Spring. Les Trichanthoe seraient caractéristiques de l'Australie occidentale, où elles seraient inégalement répandues dans divers districts, en particulier dans les terrains sablonneux. Quant aux Capitatce, elles n'existeraient que dans les environs du King George's Sound. I.c $C$. teretifolium serait une plante caractéristique du district Warren qu'il dépasserait quelque peu ver's l'est, son aire de dispersion étant à peu près égale à celle du $C$. flexuosum; mais les deux espèces occupent des stations un peu différentes : la dernière recherchant l'ombre, s'étend moins ver's l'est de la forêt et pénètre plus avant dans l'intérieur' des terres.

Le tableau suivant des espèces du genre, avec leur dispersion som- 
mairc. perinettra de se rendre compte des groupcurents proposés par les anteu's allemands, et de jugor de la distribution des nombreux Conospermum.

\section{CONOSPERMUM Sm.}

Section Caprat.1. - C. capitalum R. Br. - Australic occidentale. C. petiolare R. IBr.

Section 'Teretroli.e. - C. teretifolium R. Br.

section Isomerum. - * C. flexuosum R. Br.

Section Euconospermum.

Sub-section Foliosa. -- C. acerosum Lindl.

C. Croninice Diels

C. aminum Meisn.

C. nervosum Meisn.

C. diffusum Bentl.

C. glumaceum Lindl.

Sub-section Nuda. - C. ephedroides Kipp.

C. Toddii F. v. Muell.

C. polycephalum Meisı.

C. leianthum Bienth.

C. Eatonice E. Pritzel

C. coruleum R. Br.

C. delile Kipp.

C. scaposum Benth.

* C. Huegelii R. Br.

C. densiflorum Lindl

* C. Brownei Meisu.

C. longifolium $\mathrm{Sm}$. - Nouvelle falles du Sut.

Sub-section Corymbosa. - C. tenuifolium R. Br. - Nouvelle Galle du Sud.

C. Mitchellii Meisn. - Australie méridionale, Victoria.

C. sphacelatum Hook - Queensland, Australie septentrionale.

C. patens Schlecht. - Australie méridionale, Victoria, Nourelle Galle du Sud.

C. taxifolium Sm - Tasmanie, Victoria, Nouvelle Galle du Sud, Queensland.

C. ericifolium $\mathrm{Sm}$. - Nouvelle Galle du Sud.

C. cllipticum Sm. Nouvelle Galle du Sud. 
Section Truchanti... - C. distichum R. Br. - Australie occident.
* C. floribundum Benth.
* C. incurem Lind.
C. brachyphyllum Lindl.
C. stcechadis Endl.
C. triplinervium $\mathrm{R} \mathrm{Bl}^{\text {. }}$
C. bracteosum Meisn.
C. crossincrvium Meisu.

Nous avous pu, dans les planches ci-jointes, faire dessiner cinq espèces de ce genre dont il n'existe jusqu'à présent que peu de figures; nous les avons fait prẻcéder d'une astérisque dans la liste précédente; elles appartiennent à trois des cinq sections proposées par MM. Diels et Pritzel.

\section{Conospermum Brownei Meisu.}

in I'l Preiss. II (I844) p. 248 et in DC. Prodr. regn, veget. XIV p. 324;

Bentham Fl austral. V p. 370.

Petite plante huissonnante, à base ligneuse, à tige florale feuillue à la hase, glabre et glaucescente. Feuilles lancéolées, cunéiformes ou obovales-oblongues, subaiguës au sommet, de 2.5 à $5 \mathrm{~cm}$. de long, rétrécies en court pétiole, munies de 3 nervures principales, les latérales moins prononcées que la médiane et atteignant environ le milieu du

Explication des figures $1-10$ de la planche XXXI.

Conospernum Brownei Meissn.

Fici. 1. - Rameau fleuri (1/1).

Fig. 2. - Bouton (7/1).

Fir. 3 - Corolle epanouie avec la bractee basilaire (7/1)

Fir: 4 - Braclee Horale vue de dos $(8 / 1)$.

Fis. 5. - Corolle fendue el étalée (8/1).

Fili. li - litamine complete (1H/1).

Fig 7 . - Litanine à une anthère et un stylet (14/1).

$F_{l ;}$ S. - Staminode à deux stylets (14/1).

FIG. 9. - Ovaire, style et stigmate 10/1).

FIG. 10. - Coupe longitudinale de l'ovaire (14/1). 
limbe. Pédoncule terminal atteignant $30 \mathrm{~cm}$. de lonğ, simple ou ramifié au sommet, formant un corymbe on une panicule compacte constituée de pelits épis. Fleurs ì braktées ovales-aiguës, glahres sauf à la base, plus courtes que le tube du périanthe; tube légèrement renflé au sommet, à limbe bilobé, à lobe postérieur cucullé, entier, arrondi, plus court que le lobe antérieur, abritant me étamine complète à lobe antérieur trilobulé, à lobules de la moitié environ de la longueur du lobe, subégaux et subaigus au sommet. Étamine médiane réduite au filet, surmonté de deux filets divergents; étamine latérale a une loge. Ovaire ovoïde, velu sur toute sa surface, à poils relativement courts sur les parois latérales, à poils plus longs que l'ovaire sur la face supérieure. Style grêle irrégulièrement contourné au sommet, sélargissant, à stigmate muni d'une fente longitudinale.

\footnotetext{
Hab - District Avon (Pritzel) près Waeel: près de Mount Moore (King et Lefroy); District de Coolgardie près Ghooli (Pritzel).
}

Observations. - Le C. Brownei se range. comme on peut le voir d'après la liste des espèces, que nous avons donnée plus haut, dans la section Euconospermum prise dans son sens strict, c'est-à-dire tel que le comprennent MM. Diels et Pritzel. Dans cette section r'éduite, on peut considérer trois groupes en se basant sur les caractères tirés des tiges florifères, comme l'avait déjà proposé Bentham dans la F'lore d'Australie. Ces trois groupements se caractérisent, d'après le savant botaniste anglais, comme suit :

Foliosae. - Tiges feuillues jusqu'à l'inflorescence. Épis non en corymbes.

Nudae. - 'Tiges feuillues à la base seulement. Pédoncules terminaux simples ou paniculés, non feuillus.

Corymbosae. - T'iges feuillues jusqu'à l'inflorescence. Pédoncules nombreux terminaux ou axillaires, portant chacun plusieurs épis, formant dans leur ensemble une panicule corymbiforme.

Au point de vue de la distribution géographique ces sous-sections sont assez nettement caractérisées; la première et la seconde se rencontrent dans l'Australie occidentale; la troisième est plus spéciale à l'Australie orientale. 
Un eoup d'œil jeté sur les figures $1-10$ de la planche, font voir que l'espèce liguréc apprartient bien à la sous-sectioll que nous avons dénommée Vudce. Ce groupement l'enferme ainsi onze espèces parmi lesquelles le $C$. densiflorum Lindl., le $C$. Brownei Meissn. et le C. longifolium Sm. forment un trio earactérisé par des fleurs disposées en nombreux petits épis constituant par leur ensemble une panicule corymbiforme compacte terıninant un long pédoncule nu Tandis que che\% le $C$. densiflorum les feuilles sont filiformes velues, à longs poils, que cher le $C$. longifolnum elles sont lancéolées, oblongues-lancéolées ou linéaires, mesurant de 7 à $15 \mathrm{~cm}$. de long, - elles sont, chez la plante qui nous occupe, comme le fait roir très bien notre figurc 1 , obovales oblongues, glabres, et n'atteignent jamais $5 \mathrm{~cm}$. de long.

La plante figuréc a été recueillie par le $\mathrm{D}^{\mathrm{r}}$ Pritzel, dans les r'égions sablonneuses du District Aron, en 1901.

\section{Conospermum floribundum Benth.}

Fl. Austral. V (1870) p. 373.

Conospermum distichum Meisn. in Pl. Preiss. I (1844) p. 522 et in DC. Prod. regn. veget. XIV, p. 318, par R. Br.

Petite plante buissonnante atteignant $1 \mathrm{~m}$. de hauteur, plus ou moins argentée, ramifiée. Feuilles rapprochées le long des extrémités des ramifications, linéaires, subcylindriques, étalées-recourbées, redressées ver's leur extrémité. de 10 à $18 \mathrm{~mm}$. de long. Inflorescences axillaires se déve-

\section{Explication des figures 11-18 de la planche XXXI}

\section{Conospermum floribundum Benth.}

Fia. 11. - Rameau florifère (1/2).

FIG. 12. - Bractée florale vue le dos $(7 / 1)$.

Fig. 13. - Fleur èpanouie (9/1)

Fig. 14. - Corolle fendue et étalée (\$/11).

FIG 15. - Étamine complète (15/1).

FiG. 16. - Étamine à une anthère et un filet (15/1).

FIG. 17, - Staminode à deux stylets bifides au sommet (15/1)

FiG. 18. - Ovaire, styie et stigmate $(8,1)$. 
loppant ì l'aisselle des fenilles supérieures, formant des épis de 2,5 à $5 \mathrm{~cm}$. de long, solitaires, mais forment par leur ensemble une panicule plus ou inoins compacte. Bractées florales ovales-aiguës, glabres, sauf ‘̀ la base. Fleur's à périanthe subcylindrique de $8 \mathrm{~mm}$. environ de long, légèrement enflé à la base, non contracté au sommet, blanchâtre, densément velu-cotonneux, bilabié, à lobe postérieur ovale-aigu, cucullé, abritant une étamine complète, à lobe antérieur trilobulé, à lobules relativement courts, subaigus Ovaire ovoïde courtement velu latéralement, muni au sommet de poils 2 à 3 fois aussi longs que lui ; style grêle replié au sommet, terminé par un stigmate élargi, subcylindrique, fendu longitudinalement.

\section{Hab. - District North West Plantagenet (Pritzel); Swan River (Drummond); Stirling Range (F. v. Muell.).}

Observations. - Le C. floribundum Benth. comme le C. incurvum Lindl, appartient au groupe des Euconospermum dont les auteurs allemands ont fait la section Trichantce. D'après Bentham ce groupement se caractérise par les fleur's densément velues-cotonneuses; parmi les lıuit espèces de ce groupe, toutes endémiques dans l'Australie occidentale, les cinq premières se signalent par leurs feuilles cylindriques ou subcylindriques.

Chez les $C$. distichum et floribundum les inflorescences sont axillaires et simples, logées dans les aisselles des feuilles supérieures des ramifications de la tige, tandis que chez les 3 autres espèces les fleurs sont disposées en racèmes ou en panicules. La différenciation de ces deux espèces repose sur la longueur des fenilles; tandis que ehez le $C$. distichum ces feuilles mesurent de 3,5 et $7,5 \mathrm{~cm}$ de long, dans la plante qui nous occupe elles atteignent au maximum $18 \mathrm{~mm}$. de long.

Les échantillons qui ont servi à nos analyses, ont été r'ecueillies dans les régions sablonneuses du District North-West Plantagenet par M. le $\mathrm{D}^{\mathrm{r}}$ Pritzel, - soit dans une zone où jusqu'à ce jour la plante n'avait pas encore été signalée. 


\section{Conospermum incurvum Lindl.}

Swan River App. (1840) p. 30; Meisn. in Pl. Preiss. I p. 5*3 et II p. 250 et in $D C$. Prod. regn. veget. XIV p. 3I5; Benth. Fl. Austral. V p. 373 .

Plante buissonnante dressée, légèrement pubescente le long des tiges. Feuilles glabres, assez rapprochées, linéaires, subcylindriques, étalées-dressées, recuurbées vers le sommet, de 1,2 à $5 \mathrm{~cm}$. de long. Intlorescence terminale à pédoncule pouvant atteindre $25 \mathrm{~cm}$. de long, muni sur toute sa longueur de petites bractées lancéolées-subulées, et plus ou moins ramifiée au sommet, à épillets plus ou moins nombreux, rapplochés, atteignant au maximum environ $3 \mathrm{~cm}$. de long. Bractées florales, largement ovales-aiguës, velues à la base. Fleurs à périanthe de 6 à $8 \mathrm{~mm}$. de long, subcylindrique, non contracté vers le sommet, densément velu cotonneux et blanchâtre extérieurement, bilabié au sommet, à lèvre postérieure ovalesubaiguë, cucullée, abritant une étamine complète, à lèvre antérieure environ aussi longue que la lèvre postérieure, trilobulée au sommet, à lobules de la moitié environ de la longueur de la lèvre. Étamine médiane réduite au filet terminé par deux stylets; étamines listérales à une loge.

\section{Explication des figures 19-27 de la planche XXXI.}

Conospermum incurvum Lindl.

FıG. 19. - Rameau florifère (1/1).

Fig, 30. - Bractée florale vue de dos (5/1).

FIG. 21. - Fleur épanouie (5/1).

Fıa. 22. - Corolle fendue et étalée, on a laissé en place, au centre, l'ovaire avec style (1/1).

F1a. 23. - litamine complète (12/1).

Fıg. 24. - Étamine à une anthère et un stylet (12/1).

Fig. 25. - Staminode à deux stylets denticulés vers le sommet (12/1).

FiG. 26. - Ovaire, style et stigmate (9/1).

Fig. 27. - Coupe longitudinale de l'ovaire (9/1). 
Ovaire ovoïle lígiremont velı à la hase, glabre sur les parois latérales et muni an sommet d'mu ammean de poils ulus lougs que lui. Sty]r groble plusieurs fois plus long que l'ovaire, ondulé vers le sommet, s'élaruissant au sommet en un stigmate subcylindrique, fendu longitudinalement.

Hab. - District Avon près de Mount Caroline et de Tammin (Pritzel); District Irwin près de l'Irwin River (Pritzel): Swan River (Drummond).

Observations. - Le C. incurvum se range comme nous l'avons signalé à propos de l'espèce précédente dans la section Trichantce, caractérisée par ses lleur's densément velues cotonneuses. Comme le c. floribundum, la plante qui nous occupe possède des feuilles cylindriques on semi-cylindriques et appartient donc an même sous-groupe; mais tandis que chez le $C$. foribundum les fleurs sont disposées en épis simples et axillaires, dans la plante figurée planche XXXI, figure 19 , l'inflorescence forme une sorte d'épis racèmeux. terminant un pédoncule assez allongé. Trois espèces possèdent ce caractère, ce sont les $C$. incurvum Lindl., C. brachyphyllum Lindl. et C. staechrdis Endl. Cette dernière espèce possède des feuilles atteignant $14 \mathrm{~cm}$. de long, les plus petites mesurant $7 \mathrm{~cm}$., dans les deux autres les feuilles mesurent 1,2 à $7,5 \mathrm{~cm}$. de long. Les mensurations données par la Flora Australiensis pour l'espece qui nous occupe varient de 1,2 à $2,4 \mathrm{~cm}$. alors que dans les échantillons ayant servi de modèle pour les figures de la planche les feuilles mesurent jusque $5 \mathrm{~cm}$. de long. Bentham d'ailleurs considérait le C. brachyphyllum comme très variable et comme constituant peutêtre une simple variété dont les feuilles seraient plus longues que celles du typique $C$. incurvum.

La plante figurée provient des récoltes du $\mathrm{D}^{\mathrm{r}}$ Pritzel dans les régions sablonneuses du district Avon, dans lequel cette plante ne paraissait pas encore avoir été recueillie.

\section{Conospermum Huegelli $R . B r$.}

in Endl. Nov. Stirp. Dec. (1839) 58; Bentham Fl. Austral, V p. 369.

Plante petite, vivace, glabre sauf sur l'inflorescence. Feuilles basilaires formant une touffe assez épaisse au sommet de la courte tige, linéaires-subulées, de 2,5,57 cm. de long et atteignant au maximum $2 \mathrm{~mm}$. de diamètre. 
Inflorescences terminales, à pédoncule dressé, non ramifié, privé de feuille, atteignant parfois plus de $50 \mathrm{~cm}$. de long, portant à son sommet un épi court, plus ou moins ovoïde. Fleurs bleuâtres, munies chacune d'une bractée ovale élargie, acuminée, velue légèrement à la base. comme le rachis de l'inflorescence, et entourant tout à fait la base du périanthe, étant dans sa partie médiane environ aussi longue que lui. Périanthe glabre, d'environ $7 \mathrm{~mm}$. de long, à limbe bilobé environ aussi long que le tube ou un peu plus court, à lobe antérieur ovale-cucullé, à lobe postérieur trilobé au sommet à lobules du tiers environ de la longueur totale du lobe. Étamines insérées à la gorge du périanthe, l'antérieure complète, les latérales réduites à une anthère, la postérieure réduite au filet terminé par deux stylets divergents. Ovaire ovoïde-tronqué, fortement velu à la base, moins fortement le long des parois latérales, muni au sommet d'un cercle de poils dressés, plus longs que l'ovaire. Style grêle, ondulé, à stigmate presque aussi long que le style, plus ou moins élargi.

Hab. - District Swan (Pritzel); Swan River (Huegel et autres).

Observations. - Le Conospermum Huegelii appartient, ainsi que le montre le tableau que nous avons donné dans la notice sur le genre, au sous-genre ou section Euconospermum pris dans le sens strict des botanistes allemands et aussi à la série des Euconospermum que Bentham a

Explication des figures $1-9$ de la planche XXXII.

Conospermum Huegelli $R$. $\mathrm{Br}$.

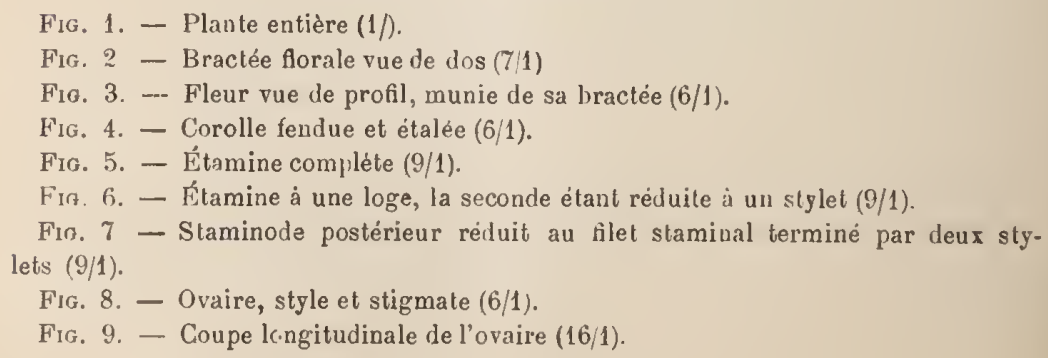


caractériséc par des fleurs glabres on pubescentes, mais non cotonneuses. Ainsi délimité le groupement des Euconospermum pout ètre subdivisé en trois subdivisions, dont les caractères se résument comme nous l'avons indiqué à propos du $C$. Brownei, espèce plus ou moins affine du $C$. Inuegelii; mais tandis que cher le $C$. Brownei et les espèces voisines les fleur's sont réunies au sommet d'un long pédoncule en unc panicule corymbiforme compacte, che\% les C. Huegelii, C. scaposum Benth. et $C$. densiflorum Lindl, l'épi floral est compacte non corymbiforme. Les différences entre ces trois dernières espèces est facile à saisir, la nature des feuilles suffisant pour la différenciation : che\% le $C$. scaposum les feuilles sont oblancéolées, couvertes de longs poils étalćs; chez lc $C$. densiflorum elles sont filiformes et également munies de longs poils étalés; par contre, commo le montre très nettement la figure 1 de notre planche XXXII, les feuilles du $C$. Huegelii sont étroitement linéaires ou subulées et glabres et cc derniel caractère à lui scul suffit donc déja pour permettre la séparation spécifique de ces trois formes.

La plante figuréc a été recueillie par M. E. Pritzel dans le Swan district, dans les forêts ombreuses du Darling Range; soit dans la même l'égion de la Swan Rivel' où Huegel l'avait primitivement rencontr'ć et où Drummond et Preiss. l'avaient revue après lui.

\section{Conospermum flexuosum $R . B r$.}

Prot. nov. (1830) p. II ; Meisn. in Pl. Preiss. I p. 526;, II p. 251 et in DC. Prod. l'egn. veget. XIV p. 324 ; Bentham Fl. Austral. V p. 366.

Plante sous-buissonnante atteignant 1,25 m. de hauteur, composée en grande partie l'inflorescence. Feuilles toutes radicales ou subradicales, longuement lancéolées, rétrécies à la base en un pétiole plus ou moins allongé, subobtuses au sommet, légèrement apiculées atteignant $30 \mathrm{~cm}$. de long, pétiole compris, à nervure médiane assez nette, nervures latérales anastomosées avant d'atteindre le bord, constituant une nervure intramarginale nette mais peu proéminente. Inflorescence paniculée, à ramifications divariquées, flexueuses, munies au niveau des ramifications anguleuses de bractées aiguës. Fleurs petites, blanchâtres, courtement velues, leéunies à l'extrémité des 
ramifications en petits épis ou en capitules de 2 à l() fleurs. Bractées florales ovales élaroies, entourant totalement la base de la fleur, subaiguës, velues éparsement sur le dos, presque aussi longues que le tube du périanthe. Celui-ci de $2 \mathrm{~mm}$. environ de long, légèrement renflé au niveau des étamines, surtout du côté postérieur; lobes subégaux, étalés-dressés, oblongs-lancéolés, de 3 à $4 \mathrm{~mm}$. de long, le postérieur un peu plus développé que les autres. Étamines insérées dans le tube du périanthe apposées au lobe; étamines postérieures réduites au filet, terminé par deux styleis divergents, les latérales ne portant qu'une anthère, l'antérieure complète. Ovaire ovoïde-tronqué, densément velu, à poils également distribués, à ovule pendant, terminé par un style recourbé, élargi en cornet, fendu dans sa moitié supérieure

Hab. - District Warren (Pritzel): King Georg's Sound et districts voisins (divers ; district South West Plantagenet (Pritzel): Cape Naturaliste et Vasse River (Oldfield).

Observations. - Comme nous l'avons dit en examinant sommairement le genre Conospermum, cette espèce que Bentham considérait dans la Flora australiensis comme appartenant au sous-genre ou section Isomerum, devrait constituer une section propre, sur laquelle les auteur's allemands, MM. Diels et Pritzel n'ont pas insisté.

Considérant cette section Isomerum telle que nous en avons relevé les

Explication des figures 10-19 de la planche XXXII.

Conospermum flexuosum $R$, $l 3 r$.

Fig. 10, - Base de la plante avec une parlie de l'inflorescence (1/1).

Fig 11. - Bractée fiorale vue de dos (8/1).

Fıg. 12 - Fleur vue de profil, entouree à la base par sa bracté (10/1).

Fif. 13 - Corolie fendue et étalée (8/1).

FıG. 14. - lítamine complèle (18/1).

FIG 15. - ĺtamine à une loge $(181)$.

Fig. 16. - Staminode postérieur réduit au tilet, terminé par deux stylels (18/1).

Fig. 17 - Ovaire avec style vu de face $(8 / 1)$.

Fis. 18. -- Ovaire avec stgle vu de profil $(8 / 1)$.

FIG 19. - Coupe longitudinale de l'ovaire (12/1). 
caractères plus liant, nous pouvons la comparel de plus près avec les especes voisines des sections Cupitate et Teretifolia. Comme le nom de la section Capitatce l'indique déjà, les $C$. capitatum et $C$. petiolare se caractérisent par leur's inflorescences qui forment des capitules denses et ces capitules sont sessiles a la base de longues feuilles terminant une tige courte. Quant aux C. teretifolium et au C. flexuosum, dont nous donnons les ligures analytiques dans la plauche ci-contre, ils ont comme caractère cominun de posséder des inflorescences paniculées, ainsi que le montre la flgure 10 de la planche XXXII.

La différence entre les deux espèces plus ou moins voisines réside dans la forme des feuilles et dans celle de l'inflorescence. Ainsi que la dénomination proposée dans le tableau ci-dessus le fait pressentir, les feuilles sont che\% le $C$. teretifolium, cylindriques; quant aux fleurs elles forment une panicule corymbiforme compacte, à fleur's dont le périanthe atteint $18 \mathrm{~mm}$. de long. Che\% le C. flexuosum, notre planche le fait très bien ressortir, les feuilles sont planes, linéaires ou lancéolées, et les lleurs forment une panicule flexueuse, à rameaux divariqués, les ramifications ultimes étant terminées par les épillets de fleur's dont le périanthe atteint au maximum $8 \mathrm{~mm}$. de long. Cette inflorescence ayant un très grand développement, nous n'en avons ju représenter qu'un fragment sur notre planclie.

Les matériaux ayant servi à figurer l'espèce, ont été récoltés dans les forets sablomneuses du district " Soutlı West Plantagenet “. 



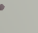





\section{PLANTA NOV E}

VEL MINUS COGNIT $Æ$

EX IIERBARIO HOR'TI THENENSIS

\section{PLANTES NOUVELLES OU PEU CONNUES}

CONTENUES DANS L'HERBIER

DE

L'HORTUS THENENSIS

AVEC LES DESCRIPTIONS OU ANNOTATIONS

DE

M. Em. DE WILDEMAN

DOCTEUR EN SCIENCES

CONSERVATLUR AU JARDIN BOTANIQUE DE L'ÉTAT

\section{Cincuiène livraison.}

(DÉCEMBRE 1905)

VEUVE MONNOM

IMI I IMEUIR F'T HIDIIHUIR

32, RUE DE L'INDUSTRIE, 32

BRUXELLES

Se vend exclusivement à l'Administration des biens et affaires de M. van den Bossche, 5, Grande Montagne, à Tirlemont. 



\section{CINQUIEME LIVRAISON}

Cette livraison des Planle Nore dome les figures et la description de sept espèces nouvelles, appartenant à des familles différentes (Hydrocharilacer, Liliacer, Iridacee?, Oxalidacece, T'iliacece et Rubiacea).

Cornme la première et la troisième, elle a été composée it l'aide de matériaux tirés de cutte section de notre herbier qui renferme les plantes récoltées au Mozambique par M. Ed. Lıja.

L. B. 

PL iovae Herb Hort Thenensis

$P_{L} X X X I I I$

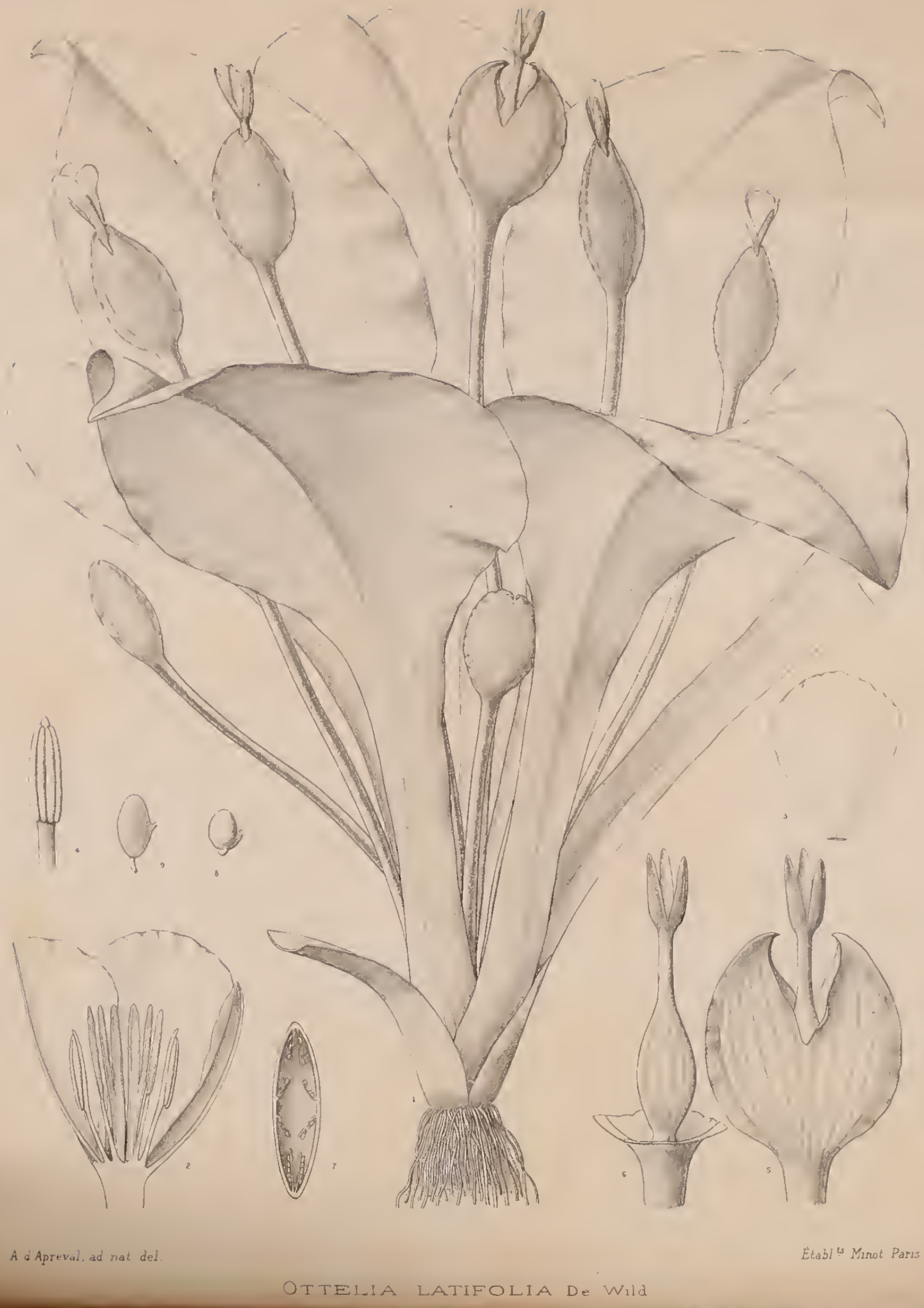




\title{
Ottelia latifolia De Wild. nov. sp.
}

\author{
(HYDROCHARITACEA)
}

Ottelia latifolia; acaulis; foliis late ovatis in petiolo attenuatis, $28-30 \mathrm{~cm}$. longis et $8-11 \mathrm{~cm}$. latis; scapis $[5-23 \mathrm{~cm}$. longis, plus minus compressis; spatha uniflora inflata, subcompressa oblongo-ovoidea, extus vittata, $30 \mathrm{~mm}$. circ. longa et $20 \mathrm{~mm}$. lata bidentata; flore spatham superante, sepalis 3 oblongo-lanceolatis, subacutis, circ. $13 \mathrm{~mm}$. longis et $3 \mathrm{~mm}$. latis, viridibus; petalis 3 luteis, obovatis, undulatis, circ. $14 \mathrm{~mm}$. longis et $6-7 \mathrm{~mm}$. latis; staminibus 3?, antheris 2 , stylis 6 , bifidis; ovario uniloculario, placentis 6 ; fructibus in spathe persistante inclusis.

Plante herbacée, acaule à feuilles radicales de 28-32 cm. de long et 9-11 cm. de large, entières, membraneuses, largement ovales, subaiguës au sommet, rétrécies vers la base en un pétiole large, plus au moins embrassant à liı base, atteignant $15 \mathrm{~mm}$. de diamètre. Scapes plus courtes ou environ aussi longues que les feuilles, de $15-23 \mathrm{~cm}$. de long, plus ou moins comprimées. Spathe renflée, comprimée, ovoïde-oblongue, de $3 \mathrm{~cm}$. de long et $2 \mathrm{~cm}$. de lirge, striée longitudinalement, à ailes ondulées, plus ou moins profondément bidentées au sonnmet, se lacérant irrégulière-

Explication des figures de la planche XXXIII.

Fli: 1. - Plante fleurie et fructifère (1/1).

FIG. 2. -- Coupe longitudinale de la partie supérieure de la fleur (7/1).

Fı, 3. - Pétale isolé (5/1).

F10. 4. - Authere jeune, vue de face (10/1). 
Inent avec l'âge. Fleur dépassant la spathe, à tube soudé a vec l'ovaire, renflé, aplati à la base, cylindrique vers le sommet, surmonté de 3 lobes verts, de $13 \mathrm{~mm}$. de long et 3 min. environ de large, subobtus. Pétales alı nombre de 3 , jaunes, obovales, ondulés sur les bords, de $14 \mathrm{~mm}$. de long et $6.7 \mathrm{~mm}$. environ de large. Étamines au nombre de ? 3, à deux anthères, légèrement apiculées au sommet, à filet environ aussi long que l'anthère. Styles au nombre de 6, linéaires, divisés jusque vers le milieu en deux lohes. Fruits inclus dans la spathe persistante.

Hab. - Zambèze, Morrumbala, dans un canal de drainage décembre 1900 (Éd. Luja, n. 392).

Observations. - Le genre Ottelia appartient à la petite famille des Hydrocharitacées et ne renferme que peu d'espèces; en Afrique tropicale on en compte cinq : les $O$. Alismoides Pers. de la région nilienne et du district du Mozambique, - O. lancifolia Rich., décrit sur des échantillons recueillis par Quartin Dillon en Abyssinie, retrouvé dans l'Angola, l'Afrique anglaise (Blantyre) et, sous forme d'une variété fluituns, dans l'Angola (Pongo Andongo), - (). vesiculata Ridl. (Angola), -- O. plantiginea Welw. (Angola) et $O$. halogena De Wild. et Th. Dur. (Congo).

Ces diverses espèces, plus répandues probablement qu'on ne le croit, sont généralement mal connues; ce sont des plantes difficiles à préparer pour les herbiers et souvent elles nous arrivent en mauvais état et c'est là sans doute la raison pour laquelle il y a encore des désaccords sur les caractères génériques : nombre des styles et des étamines.

La plante que nous avons fait figurer et qui, ainsi que le montrent les figures très fidèles, se trouve en bon état de conservation, a le plus d'analogie avec l'O. lancifolia Rich., dont nous avons esquissé plus haut la dispersion : elle partage en effet avec cette espèce la forme de feville

FIg. 5. - Fleurs après floraison, les pétales sont tombés, le calice fructifere est entouré de la spathe $(1,5 / 1)$.

FıG. 6. - Calice fructifère dont la spathe est enlevee $(1,5 / 1)$.

Fıc. 7. - Coupe transversale de lovaire avec placentas n'atleignant par le cen$\operatorname{tre}(5 / 1)$.

Fig. 8. - Ovule (25/1).

FIG. 9. - Graine (7/1). 
non corlée à la batso mais plutôt aigmi se rétrécissant on pótiole, la spathe bifide et non à une dizaine de dents comme che\% l'O. plantuginea WelW; la scape est subcylindrique comme chez les $O$. lancifolice et halogena et non tétragone au sommet comme chez l'o, vesiculala. Mais tandis que chez l'O. halogena les styles ne sont qu'au nombre de :3, ils sont au nombre de 6 dans lil plante nouvelle comme dans l'(). Iancifolia. C'est dans la forme et les dimensions des feuilles que nous trouvons les caractères différenticls; il suffira en effet de jeter un coup d'œil sur la planehe 95 de la Flore d'Abyssinic de Richard pour juger rapidement de l'aspect différent des deux plantes.

l'O. lancifolia, comme son nom l'indique d'ailleur's, possède des feuilles elliptiques, lancéolées, atteignant au maximum $30 \mathrm{~cm}$. de long, pétiole compris, et $4 \mathrm{~cm}$. de large; la variété fluitans Ridl. a des feuilles plus étroites mesurant $30 \mathrm{~cm}$. de long sur $3 \mathrm{~cm}$. de large, tandis que chez la plante du Morrumbala, les feuilles atteignant rarement un peu plus de $30 \mathrm{~cm}$. de long, pétiole eompris, mesurent au minimum $\& \mathrm{~cm}$. de diamètre.

Nous nous permettons d'attirer l'attention sur le nombre d'étamines. trois, alors que les auteur's en signalent six; nous n'oserions toutefois insister, car nous n'avons pu analyser qu'une llcur, et peut-être se trouvait-elle former une exception. Nous le répétons, l'analyse de ces fleur's très fragiles et probablement très fugaces est loin d'ètre aisée. 




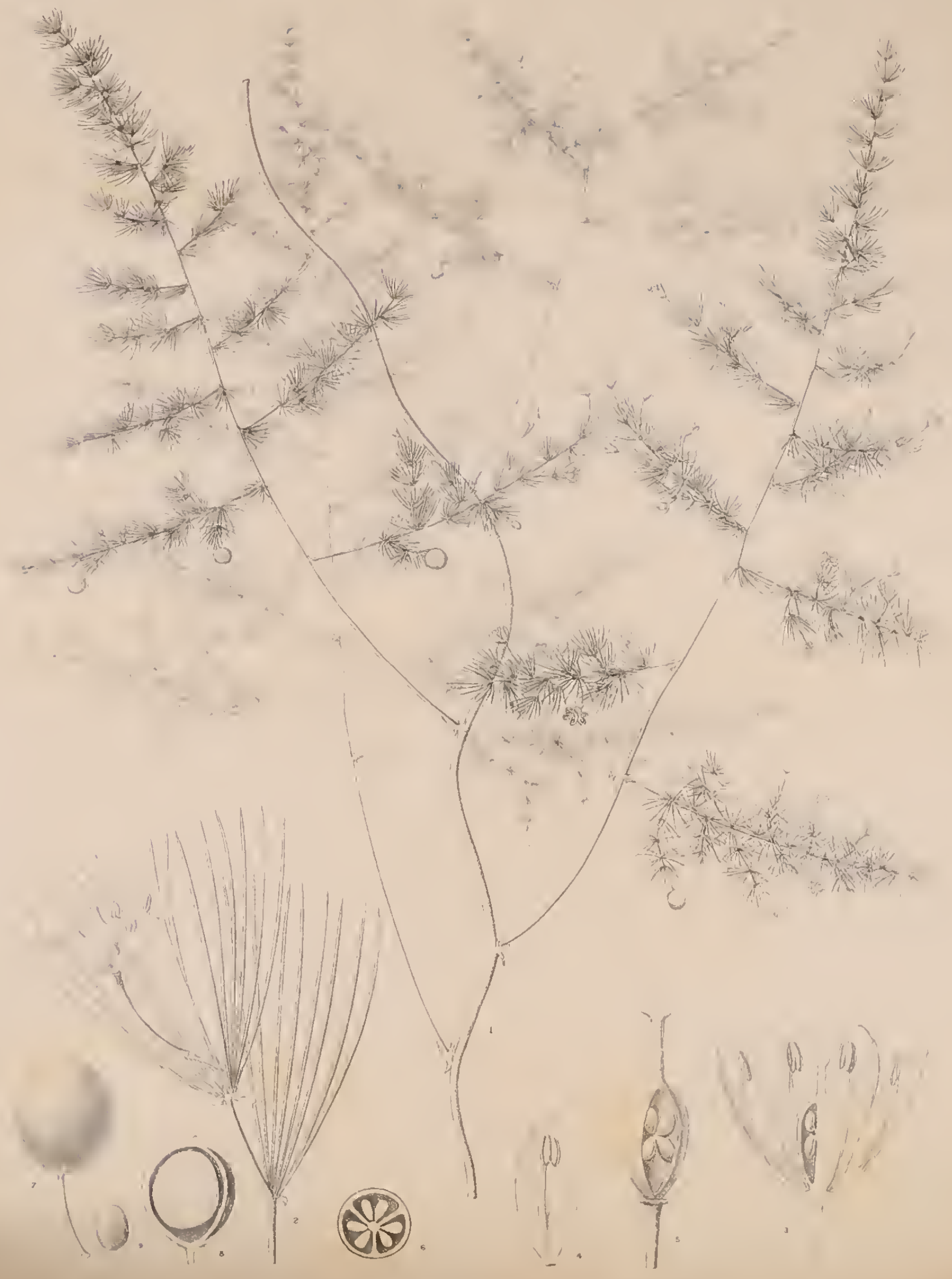




\title{
Asparagus Lujae De Wild. nov. sp.
}

\author{
(LILIACE AE)
}

Asparagus Lujae; suffrutex scandens ramosissimus, culdibus primariis teretibus lignosis, gracilibus, ramulis elongatis, strictis vel curvatis. Folia infima in spinas producta. Cladorlia 6-8 fasciculata, setacea, rigidula, falcata, gracillima, circ. $7-11 \mathrm{~mm}$. longa. Pedicelli solitarii vel geminati, 7-8 $\mathrm{mm}$. longi supra basin articulati. Perianthium circ. $3 \mathrm{~mm}$. longum. Antherae oblongae pedicello cir. triplo breviores.

Plante suffrutescente, glabre, lamifiée, à tiges cylindriques, ligneuses, grêles, à rimeaux allongés redressés, plus ou moins recourbés. Feuilles terminées à la base en un éperon aigu. Cladodes réunies par 6 à 8 en fascicules sétacés, rigides, plus ou moins falciformes, aigus, grêles, de 7-12 mm. de long. Fleurs blanches solitaires ou géminées, terminant généralement les ramuscules latéraux, à pédicelle glabre, de $7-9 \mathrm{~mm}$. de long, articulé vers le tiers inférieur. Périanthe d'environ $3 \mathrm{~mm}$. de long, à lobes obovales, plus ou moins carénés; étamines soudées à la base du pétale, à filaments aplatis un peu plus courts que le

\section{Explication des figures de la planche XXXIV.}

F1G. 1. - Rameaux avec feuilles épinescentes, cladodes et fleurs (1/1).

FIG. 2. - Fragment de tige avec cladodes et fleurs (6/1).

Fig. 3. - Coupe longitudinale d'une fleur épanouie (14/1).

Fig. 4 - Pétale isolè vu de face (12/1).

F1G. 5. - Ovaire dont une loge est éventrée pour laisser voir la disposition des ovules $(25 / 1)$. 
périanthe, a anthères oblonghtes, environ trois fois plus courtes que le filet. Ovaire glabre, ovoïde, triloculaire, à style trilobulé au sominet; fruit subglobuleux renfermant souvent une seule graine par suite d'arortement des autres oviles.

Hab. - Mozambique, forêts du Morrumbala, 16 septembre, 1900 (n. 358) et 1901 (n. 426) (Éd. Luja)

Observations. - Parmi les espéees africaines du genre Asparagus, il en est peu qui possèdent les earaetères de la plante que nous avons dédiée à son collecteur M. Luja; en effet, si nous jetons un coup d'œil sur la elef analytique proposée par M. Baker dans le volume VII de la Flora of tropical Africa, nous verrons que l'A. Luija doit se classer dans le groupement que $\mathrm{M}$. Baker caraetérise par ees mots :

Cladodes subulés; éperon piquant, fleurs solitaires ou géminées.

I)ans ee groupement l'auteur anglais forme trois sous-groupements.

Cladodes nuls ou solitaires.

Cladodes peu nombreux dans un faseieule.

cladodes assez nombreux dans un faseieule.

C'est naturellement dans ee troisième sous-groupement qu'il nous faudra interealer l'espèce nouvelle, puisque les faiseeaux renferment plus de 6 cladodes.

l)ans eette dernière division on peut eneore former trois subdivisions:

Cladodes très eourts.

Cladodes de 6 à $12 \mathrm{~mm}$. de long.

Cladodes de $122 \mathrm{~mm}$. et au-deli.

Par la longueur des cladodes l'A. Lujce se lange dans la deuxième subdivision qui renferme en Afrique tropieale deux espèces:

A deflexus Baker, de l'Angola.

4. Nelsii Sehinz, de l'Hereroland.

Fig. 6. - Coupe transversale de lovaire (25/1).

FIg. T. - Fruit $(5 / 1)$.

Fı, 8. - Coupe longitudinale du fruit, un ovule seulement s'est développé (5/1).

Fıg. 9. - Graine isolée (3/1). 
Ces deux espèces s ont: la première à rameaux glabres, la seconde à rameaux pubescents.

Ce serait done de l' $A$. deflexus que se rapprocherait le plus l'espèce nouvelle.

Mais tandis que les cladodes de 6 à $8 \mathrm{~mm}$. de long dépassent les pédicelles de 3 à $4 \mathrm{~mm}$. de long et articulés au milieu, chez l'A. Lujae les cladodes de 7 à $12 \mathrm{~mm}$. de long sont égalés ou parfois dépassés par les pédicelles mesurant de 7 à $9 \mathrm{~mm}$. et articulés vers le tiers inférieur. La longueur des pédicelles floraux et la situation de l'articulation constituent donc des caractères différentiels bien marqués.

Dans le groupe des Asparagus à cladodes de $12 \mathrm{~mm}$. de long ou plus, dans lequel on pourrait jusqu'à un certain point ranger la plante de M. Luja, se trouve une esp̣èce du même district floral, l'A. Lugardi Baker (environ du lac Ngami) dont les caractères concordent jusqu'à un certain point, mais dont les pédicelles floraux ne mesurent que $6 \mathrm{~mm}$. de long alor's que les cladodes mesurent $12 \mathrm{~mm}$. 


Pl Novae Herb. Hort Thenensis

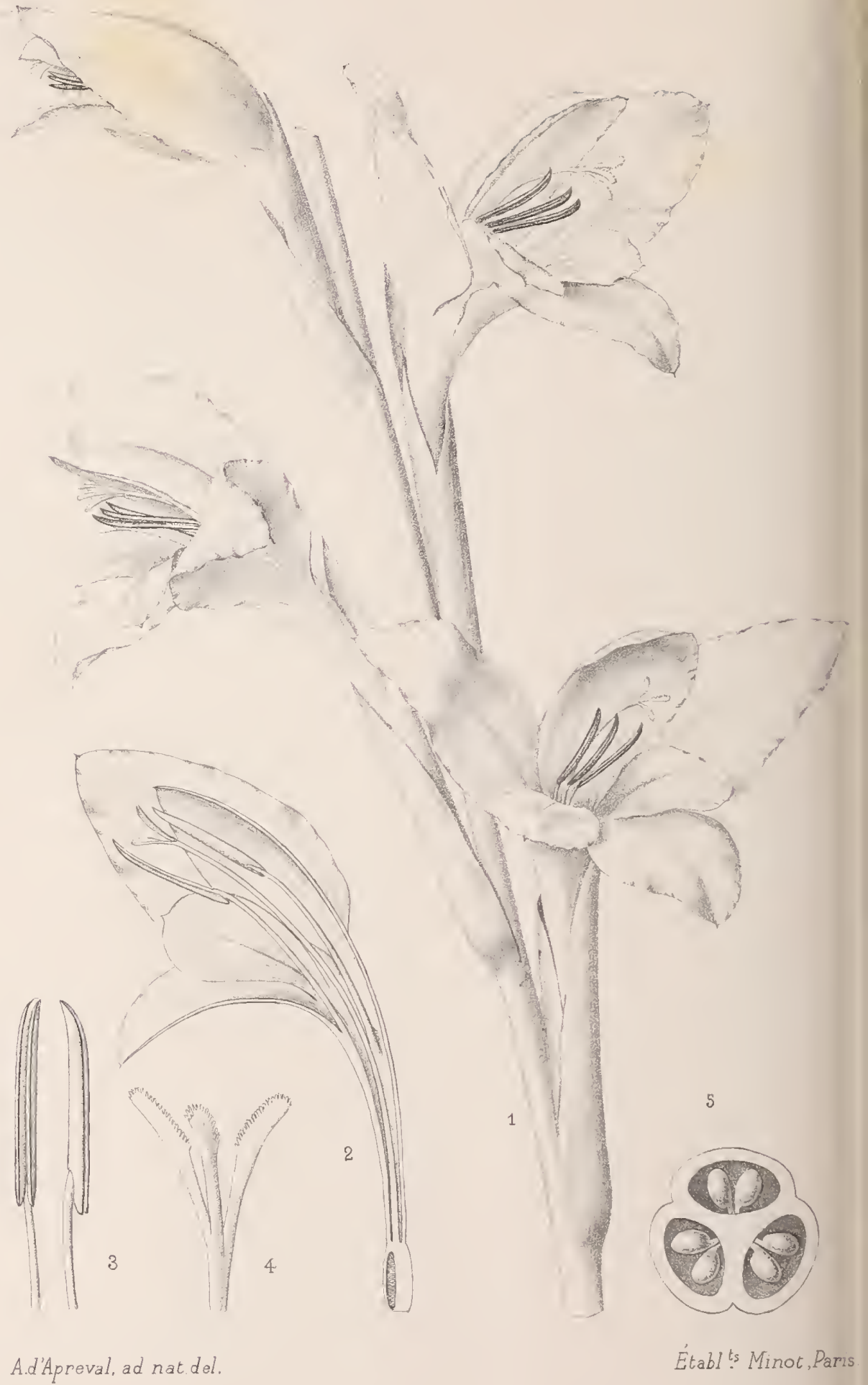

GLADIOLUS AFFinis De Wild. 


\title{
Gladiolus affinis IIe Will. nov. sp.
}

\author{
(IRII)ACHA)
}

Gladiolus affinis; elatus glabermimus, foliis ignotis; spica ultra $: 35 \mathrm{~cm}$. longa, laxiflora, floribus $4 \mathrm{~cm}$. circ. distantibus, spathis lanceolato-acutis, cire. $6 \mathrm{~cm}$. longis, tubum perianthii subaequantibus; lobis ovatis, 3-exterioribus majoribus, rubro-purpureis (sicco), stigmate trifido.

Plante dressée, glabre. Feuilles inconnues. Ėpi florifère de plus de $35 \mathrm{~cm}$. de long, laxiflore, à $7-8$ fleurs, distantes de $4 \mathrm{~cm}$. environ les unes des autres. Bractées de la spathe atu nombre de 2, entourant le tube de la fleur, l'extérieure plus longue que l'intérieure, aiquë, atteignant $6,5 \mathrm{~cm}$. de long et $16 \mathrm{~mm}$. de large. aussi longue ou légèrement plus longue que le tube du périanthe; bractée interne de 4.5 à 5 cin. environ de long. Fleurs de 8,5 à $10 \mathrm{~cm}$. de long, à tuhe dressé, droit ou légèrement recourbé, à lobes légèrement recourbés vers l'extérieur, tube et lohes d'un rouge pourpre (à l'état sec), lobes extérieurs subégaux, les latéraux étalés-dressés, ovales, subaigus, un peu plus développés que les lobes inférieurs, de $55 \mathrm{~cm}$. environ de long et $2,6 \mathrm{~cm}$. environ de diamètre; lobes internes plus courts,

Explication des figures de la planche XXXV.

FlG. 1. - Fragment de l'épi florifère (1/1)

Fic. 2. - Fleur en coupe longitudinale (1/1).

Fig. 3. - litamines vues de face et de profil (2/1). 
subobtus, le supérieur plus développé que les latéraux, légèrement cuculliforme, de $5 \mathrm{~cm}$. environ de long et d'environ $3 \mathrm{~cm}$. de large, recouvrant le style et les étamines exsertes. Anthères linéaires, biloculaires, de $14 \mathrm{~mm}$. environ de long, style dépassant légèrement les anthères, à 3 lobes obovales, glanduleux sur les bords internes.

\footnotetext{
Hab. - Mozambique, Morrumbala, décembre 1900 (Éd. Luja, n. $393^{\text {lis) }}$.
}

Observations. - Bien que nous n'ayons pas pu voir les feuilles de la plante que nous décrivons ici, nous avons cru pouvoir la rapporter dans le groupe des Engladiolus, dont nous avons indiqué les caractères en publiant dans ce recueil le $G$. Morrumbalaensis (planche V, liviaison 1), et ce dans le voisinage des $G$ corneus Oliv., Nevii Baker et splendidus Rendl., qui tous les trois d'ailleurs proviennent originairement de la même région, c'est-à-dire du district floral dı Mozambique, le premier de l'Unyamwezi (Afrique orientale allemande), le deuxième et le troisième du lílimanjaro.

C'est de la troisième de ces espèces que la plante que nous figurons ici semble se rapprocher le plus; en effet la couleur des fleur's du $G$. corneus est pâle, cornée, les fleur's du $G$. Newii sont d'un beau jaune, celle du $G$. splendidus rappellent, par leur belle couleur écarlate, les fleurs de notre $G$. affinis. Le $G$. corneus découvert par' Speke et Grant dans leur mémorable expédition en Afrique orientale est seul figuré, les trois espèces se trouvant décrites en diagnoses comparatives dans la Flora of trop. Africa VII p. 368 et 369.

I,es caractères qui nous ont amené, en l'absence d'éléments authentiques du $G$. splendidus, à faire figurer la plante du Morrumbala comme nouveauté sont: spathe plus réduite, lobe dorsal du périanthe plus étroit.

Le G. splendidus, n'a comme nous le disions plus haut, été r'encontré que par' Taylor' sur' le Kilimanjaro, au-dessus de Morang et se trouverait encore à 10,000 pieds d'altitude; la forme que nous croyons voisine et que pour cette raison nous avons dénommée affinis, est également une plante de montagne, mais d'une zone florale qui parait assez différente, située beaucoup plus au sud.

Fig. 4, - Exlrémité du style avec stigmates (4/1).

FIg. 5. - Coupe transversale de l'ovaire (6/1). 


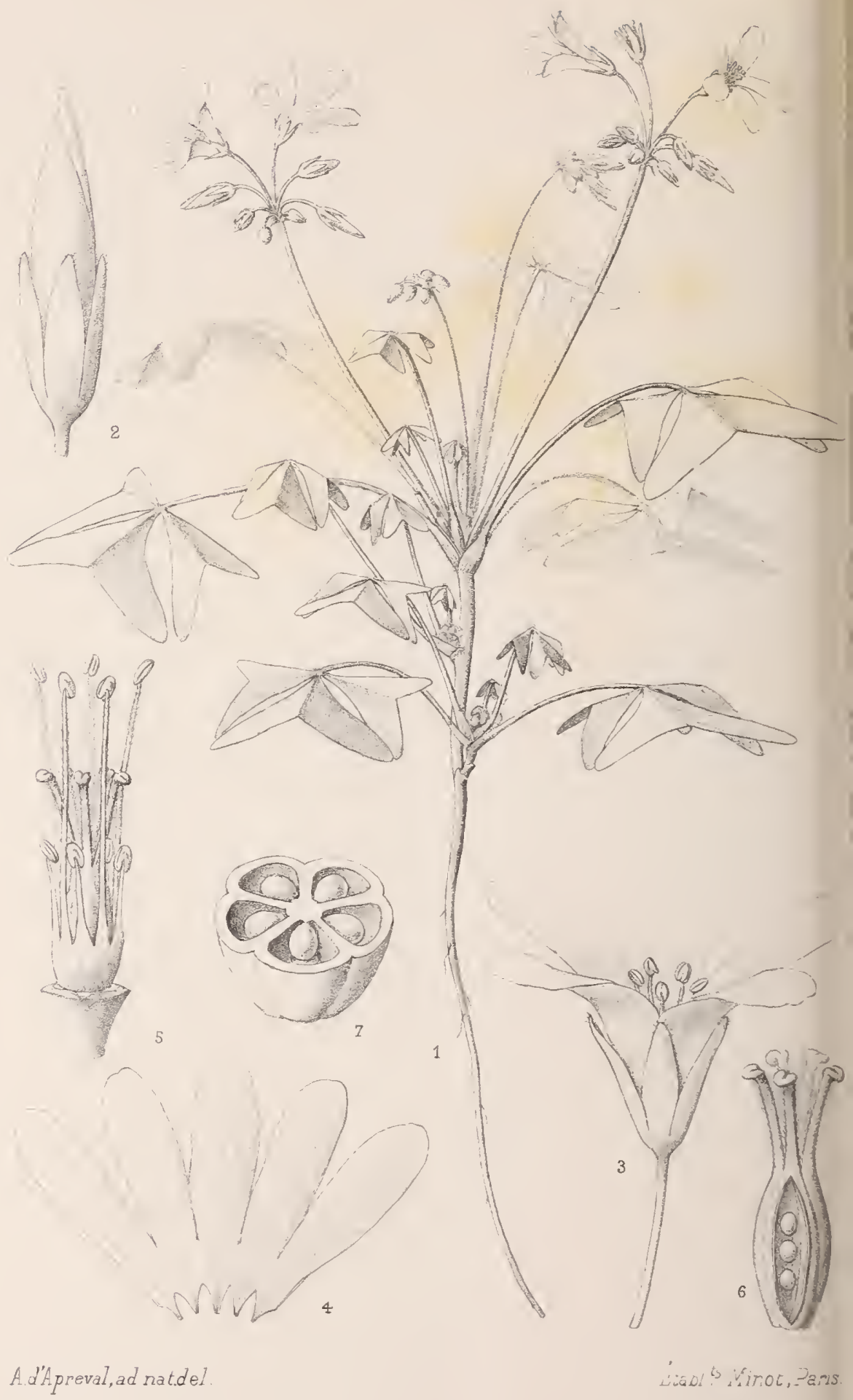

OXalis MORRUMBALAENSIS De Wild. 


\title{
Oxalis morrumbalaensis De Wild. nov. sp.
}

\author{
(OXALII)ACE,E)
}

Oxalis morrumbalaensis: herbacea, acaulescens, $14 \mathrm{~cm}$. cir'c. altal, radice elongato; foliis trifoliolatis. petiolatis, petiolo $3-5 \mathrm{~cm}$. longo, glabro vel basi dilatato sparse piloso; foliolis subpetiolulatis, triangularibus, $2-3,2 \mathrm{~cm}$. latis et $15-17 \mathrm{~mm}$. longis, apice paulo emarginatis, base anguste cuneatis, lobis lateraliter l'otundatis, nervo mediano non mucronato, supra et infra glabris, nargina sparse ciliatis; inflorescentiis subumbellatis, longepermnculatis, pedunculo $4-7 \mathrm{~cm}$. longo, glabro, umbellis 7-9-1loris, basi bracteatis, bracteis filiformibus, floribus pedicellatis, $13 \mathrm{~mm}$. circ. longis, pedicello. sparse piloso, circ. (i-12 mm. longo: calyce 4-5 mu. longo, lobis apice aurantii-callosis, extus sparse pilosis, apice ciliatis; petalis oblongis, rotundatis, circ. 12-14 11m. longis, pallide roseis, in sicco albescentibus; staninibus 10-5 brevibus, 5 longioribus, infra connatis, filanentis liberis pilosis; stylis 5 pilosis.

Plante herbacée, dressée, acaulescente, simple ou léscèrement ramifiée atteignant $14 \mathrm{~cm}$. de haut, à racine pivotante allongée. Feuilles trifoliolées, pétiolées, à pétiole de 3-5 cm. de long, glabre ou éparsement velu sur la base dila tée; folioles subpétiolées, triangulaires, de 2 à $3,2 \mathrm{~cm}$. de large et 15 à $17 \mathrm{~mm}$. de long, peu émarginées au sommet, a

\section{Explication des figures de la planche XXXVI.}

FIG. 1 - Plante fleurie (7/1).

Fic. 2. - Bouton floral (5/1).

Fir, 3, - Fleur épanouie (4/1). 
angle rentrant largement obtus, étroitement cunéiformes à la base, à lohes arrondis sur le côté, à nervure médiane non terminée en mucron; folioles glabres sur les deux faces, siuff vers la base et sur les bords oì se trouvent quelques poils épars. Inflorescences en ombelles portées au sommet de larges pédoncules, atteignant de 4 à $5 \mathrm{~cm}$. de long, glahres; ombelles à 7-9 fleurs, bractéolées à la base, à bractées fililormes, atteignant $4 \mathrm{~mm}$. de long. Fleurs pédicellées atteignant $13 \mathrm{~mm}$. de long, à pédicelle de $6-12 \mathrm{~mm}$. de long̨, épar'sement velu. Calice de 4 à $5 \mathrm{~mm}$. de long, à lobes oblongs. subobtus, munis d'un col jaune-orangé au sommet, éparsement velus on glabrescents sur le dos, courtement ciliés au sommet. Pétales rosés, oblongs arrondis an sommet, soudés dans leur partie inférieure sauf à la hase. Étamines an nombre de 10 dont 5 longues et 5 courtes, les plus longues de $6 \mathrm{~mm}$. environ de long, les plus courtes moitié aussi longues, soudées à la base en un tube unique entourant la partie inférienre de l'ovaire. à filaments velus dans leur partie libre. Ovaire glahre, quinqueloculaire, ovules sur un rang dans chaque loge, à 5 styles velus, à stigmate semi-circulaire, plus courts que les étanines longues, plus longs que les étamines courtes.

Hab. - Mozambique, Morrumbala. 20 janvier 1901, à 1,000 mètres d'altitude (Éd. Luja, n. 368).

Olservations. - Bien que le genre Oxalis, type de la famille des Oxalidacées ne renferme qu'un nombre restreint d'espèces, il n'est pas facile de les différencier, de juger de leur's caractères propres sans avoir sous les yeux, et côte à côte, les figures des feuilles de ces dirers tỵes spécifiques. La plante que nous a rapportée M. Éd Luja, de son séjour au Morrumbala appartient au groupe le plus nombreux d'Oxalis, carac-

Fia. 1 - Corolle fendue et étalée (3/1).

Fı́. 5. - Androcée el gynécée. débarrassès des enveloppes llorales (8/1).

Fıg. 6. - Ovaire, dont une loge à eléventrèe pour laisser voir la disposition des ovules (6/1)

Fig. 7. - Coupe transversale de l'ovaire (141). 
térisé par ses feuilles trifoliolées. Ic $O$. morrumbalacnsis est quasi une for'nc intermédiaire cutre les deux groupenents que l'on peut considérer. dans cettc subdivision et qui seraient caractérisés par la ramification ou la non-ramification de la tige; comme lc fait voir la planclic ci-contre, il y a dans la plante que nous figurons des ébauches de ramifications; sc seraient-elles développées plus fortement?

Nous avons décrit antéricurement un $O$. katangensis De Wild. ct Th. Dur. qui entre dans le mème groupe que celui auquel appartient pour nous l'O. morrumbalaensis, bien que la tige soit légèrement l'amifiéc; nous avons fait alor's ressortir les différences des $O$. caprina rar. abyssinica Oliv., O. semiloba Sond. et $O$. katangensis qui ont comme notre plante des fleur's de teinte rougeâtre-violacćc; I\%O. cernua Thunb. comme l'O. corniculata L. possèdle des fleurs jaunes.

Ces caractères, nous pouvons les reproduire ici en ajoutant ceux du l'o. mormumbalaensis :

\section{Folioles de $12-18 \times 17-26 \mathrm{~mm}$.}

Folioles de 10-12 × 12-18 mm. . . O. Semiloba Sond. Folioles de $7.9 \times 13-20 \mathrm{~mm}$.

$O$. katangensis De Wild. et Th. Dur.

Folioles de 15-17 $\times 20-30 \mathrm{~mm}$. O. morrumbalaensis De Wild.

Ces chiffres démontrent très clairement que la forme des feuilles de la dernière espèce est différente de celle des autres plantes citées, les rapports entre la hautcur et la largeur des folioles étant tric̀s différents. A cc caractère s'ajoutent encore des différ'cnces dans la grandeur des diverses parties de Ia fleur.

L'O. semiloba a été indiqué dans le district floral du Mozambique et méme sur le Morr'umbala où, d'après les données du D $\mathrm{D}^{\mathrm{r}}$ Kirk, son collecteur, on le rencontrerait jusqu'à 3,000 pieds d'altitude sous la forme d'une variété parviflora. Les données de la Flore du Cap et de la Flore d'Afrique tropicale ne sont pas concordantes pour cette espèce; nous arons reproduit plus haut celles de la Flora caperisis qui sont les données originales; d'après M. Olivel dans la Flora of trop. Africa I p. 296 les folioles de l' $O$. semiloba atteindraient de 18 à $36 \mathrm{~mm}$. de diamètre; elles pourraient donc être beaucoup plus développées encore qu'clles ne le sont dans la plante que nous figurons. Les fleur's de l'O. semiloba sont décrites comme pourprées et, d'après M. Luja, seraient rarcs. En présence de ces divergences d'opinion et de la ramification légèrc de la tige de notre plante, nous avons préféré la décrire comme noureautć et la faire figurer; le dessin permettra de faire ultérieurement des comparaisons qui résoudront peut-ĉtre la question de la valeur spécifique de cette plante et des espèces affines. 



$$
\text { - }
$$

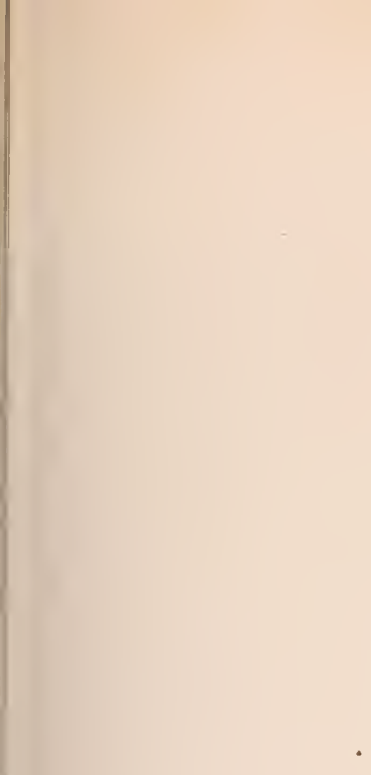

. .

.




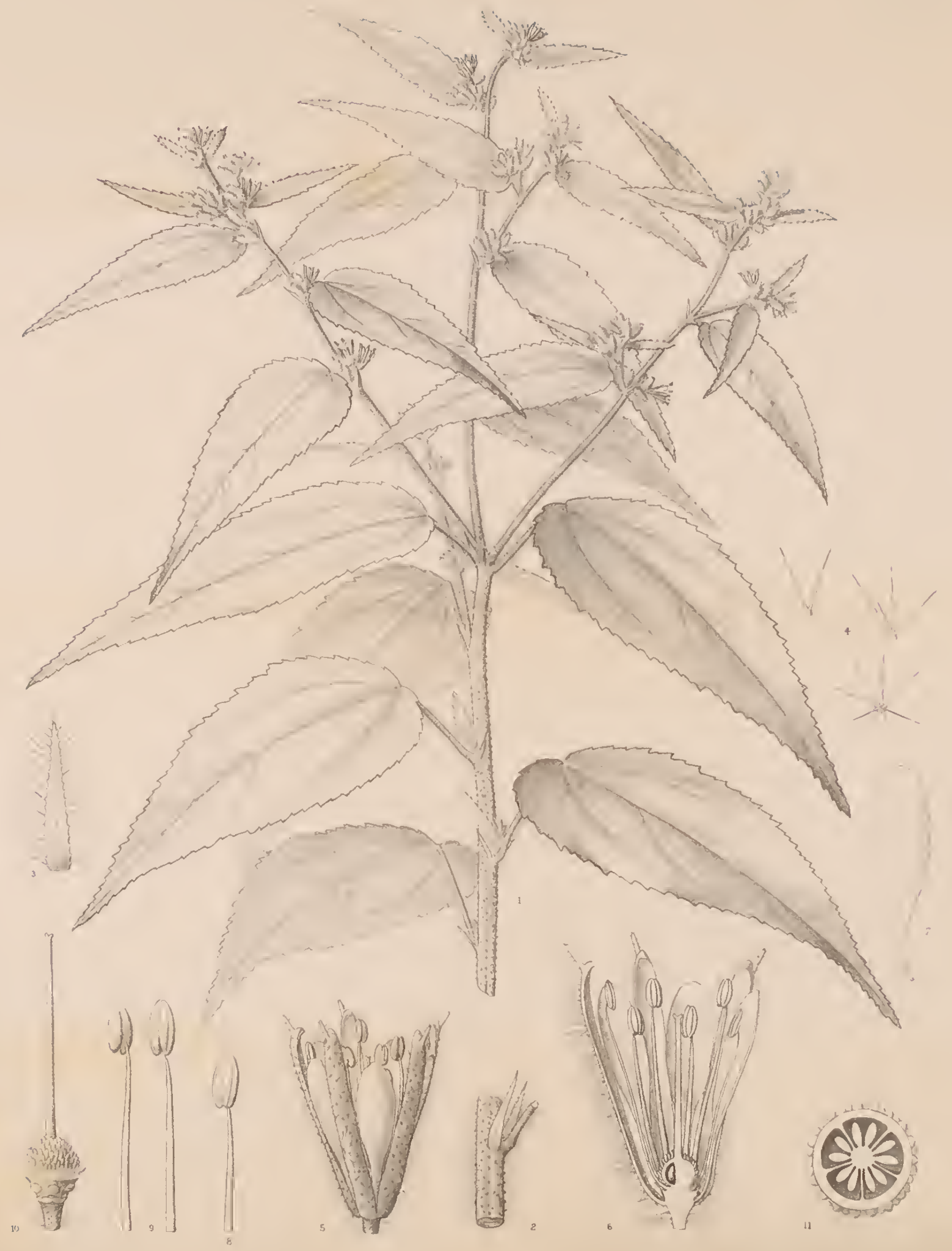




\title{
Triumfetta morrumbalana toe Wild. nov. sp.
}

\author{
(TILIACE $/$ E)
}

Triumfetta morrumbalana; ramis teretibus, tomentosis; pilis vulgo basi incrassatis, foliis modice petiolatis orato-oblongis, acutis, basi rotundatis et subcorlatis, irregulariter serratis, supra pilis simplicibus vel stellatis inspersis, subtus dense tomentosis; stipulis subulatis, univervis, piloso-ciliatis; floribus dichasia bi-triflora pedunculata referentibus, pedicellatis; sepalis anguste linearibus apice corniculatis, extus pilosis, cire. $8 \mathrm{~mm}$. longis; petalis oblongis luteo-aurantiacis, apice rotundatis, apice integris, undulatis basi puhescentibus; toro brevi quinqueglanduloso, apice membranula ciliolata terminato, ovario glaboso, glanduloso sed non piloso, stilo petala longitudine subaequante; fructibus ignotis.

Plante herbacée, de 1 à $1,30 \mathrm{~m}$. de hauteur, à tiges cylindriques, rameuses, tomenteuses, à poils brunâtres simples ou étoilés, à branches peu nombreuses, étalées, renflées à la base. l'euilles à pétiole de 2 à $3 \mathrm{~cm}$. de long à poils étalés, à limbe ovale-oblong, aigu, arrondi ou subcordé à la base, irrégulièrement denté en scie, à dents simples ou doubles, de 6 à $13 \mathrm{~cm}$. de long et de 1,5 à $4,5 \mathrm{~cm}$. de large, à poils simples ou étoilés épars sur la face supérieure, plus

\section{Explication des figures de la planche XXXVII.}

Fıg. 1. - Extrémité d'un rameau avec fleurs, vers la basēon remarque très nettement les poils renflés et étoilés, vers le haut, les poils densément étalés (3/1).

Fig. 2. - Fragment de tige avec base de pétiole et stipules (2/1).

FIG. 3. - Stipule, vue de face (\$/1). 
foncée que linférieure, densénnent tomenteuses sur la face inférieure, à deux nervures basilaires et à environ 3 nervures lintérales de chaque côté de la nervure médiane, velues-ciliées, uninerviées, de $6-8 \mathrm{~mm}$. de long. Inflorescences axillaires, se décomposant en groupes de 2 ou 3 fleurs entourées de 3 bractées lancéolćes, aiguës, ciliées, atteignant $5 \mathrm{~mm}$. de long. Fleurs courtement pédicellées, à pédicelle à poils étalés. Sépales étroitement linéaires, de $8 \mathrm{~mm}$. environ de long, munis un peu en dessous de leur sommet d'un processus corniforme, velu sur le dos. Pétales oblongs, d'un jaune-orangé, arrondis an sommet, ondulés sur le bord, entiers, rétrécis à la base en un onglet velu, de $5-6 \mathrm{~mm}$. de long. Torus court à 5 glandes, muni au sommet d'un anneau cilié; ovaire globuleux muni de glandes, non velu, à 5 loges, surmonté d'un style environ aussi long que les pétales. Fruits inconnus.

\section{Hab. - Mozambique, dans les cultures au Morrumbala, 1901 (Éd. Luja, n. 428).}

Observations. - La plante que nous venons de décrire, est sans conteste voisine de celle que le regretté prof. K. Schumann avait appelée Triumfetta abyssinica et dont il avait publié la description dans les Botanische Jahrbücher du prof. Engler. Nous avons hésité longtemps avant de créer pour la plante rapportée par M. Luja un nom nouveau, tant l'aspect des deux plantes parait semblable; cependant certains caractères extérieur's difficiles à apprécier nous disaient que la plante

FıG. 4. - Poils des différentes parties de la plante $(25 / 1)$.

Fig. 5. - Fleur épanouie (7/1).

Fig. 6. - Coupe longitudinale de la fleur épanouje (9/1).

FıG. 7. - Pétale isolé, vu de face (9/1).

FIG. 8. - Étamines oppositipétales (12/1).

FIG. 9. - Étamines oppositisépales, vues de face et de dos (12/1).

Fı́. 10. - Ovaire porté par le torus à 5 glandes et terminé par la lame ciliée, on remarque tres nettement les sortes de glandes non velues qui recouvrent l'ovaire $(12,1)$. FIg. 11. - Coupe transiersale de lovaire quinquéloculaire (35/1). 
d'Abyssinic et celle du /ambèce constituaient deux bypes bien dillérents Ia villosité est tout d'atborl un earactere chil saute aux yeux, mais smo lequel il est malheureusenent difficile de se fonder pour créer un tyje nouveau; dans le T'abyssinica, l'indument qui recouvr'c les tiges est conslitué par des poils étoilés courts, serrés, formant mu duvet appliquné sur les tiges, tandis que cher le T. mormubalance, les poils sont moins denses, mais plus lones et les poils sont simples ou ctoilés à peu de branches, l'enflés à la base et nettement étalis; ils donnent ainsi m facies tres différent au ramean. Les poils étoilés sont également beaucoup plus rares sur la face supérieure des feuilles de la plante du Zambèze que sur celle des feuilles dı T. abyssinica. On peut en outre ajouter" que la fleur de l'esprece nouvelle est un peu plus petite que celle du type de K. Schumann. Mais là ne résident pas les caractères différentiels les plus appar'ents : ils se trouvent dans la forme des pétales et dans la constitution de l'ovaire. En effet, les pétales du T. abyssinicu sont décrits comme émarginés au sommet, ceux de T. morrumbalana sont arrondis entiers; l'ovaire du $T$. aby/ssinica est r'ecouvert de sortes de glandes terminées par des poils brillants à l'état sec; l'ovair'c du T. morrumbalana possède des glandes analogues privées de poils. Il est done probable que si nous avions eu l'occasion de voir des fruits de notre plante ils auraient été complètement différents de ceux du $T$. abyssinica, qui sont recouverts d'épines droites ciliées.

En note de la description publiée par K. Srhumann (1), l'auteur croit pouvoir rapporter la plante qui a été signalée par M. Masters dans la Flora of tropical Africa sous le nom de $T$. pilosa Roth, à la plante qu'il décrit comme $T$. abyssinica, car, dit-il, le tṛpique pilosa a des épines recourbées au sommet et de telles formes n'ont pas été rencontrées dans la région.

Nous avons signalé antérieurement au Katanga sous le nom de T.pilosa, une plante dont nous n'avons malheureusement pas vu les fleurs et qui, par le caractère des épines du fruit, se rapporte très bien à cette espèce et ne peut pal' suite être confondue avce le $T$. abyssinica. Peut-être la forme du Katanga serait-ellc à rapporter plus tar'd à celle du Zambèse, bien que les caractères tirćs de la vestiture des tiges et des feuilles ne concordent pas complètement, pas plus d'ailleurs que l'absence de poils sur l'ovaire, caractère particulier de notre espèce nouvelle.

(1) in Englev. Bot. Jahrb. XV (1893), p. 127. 




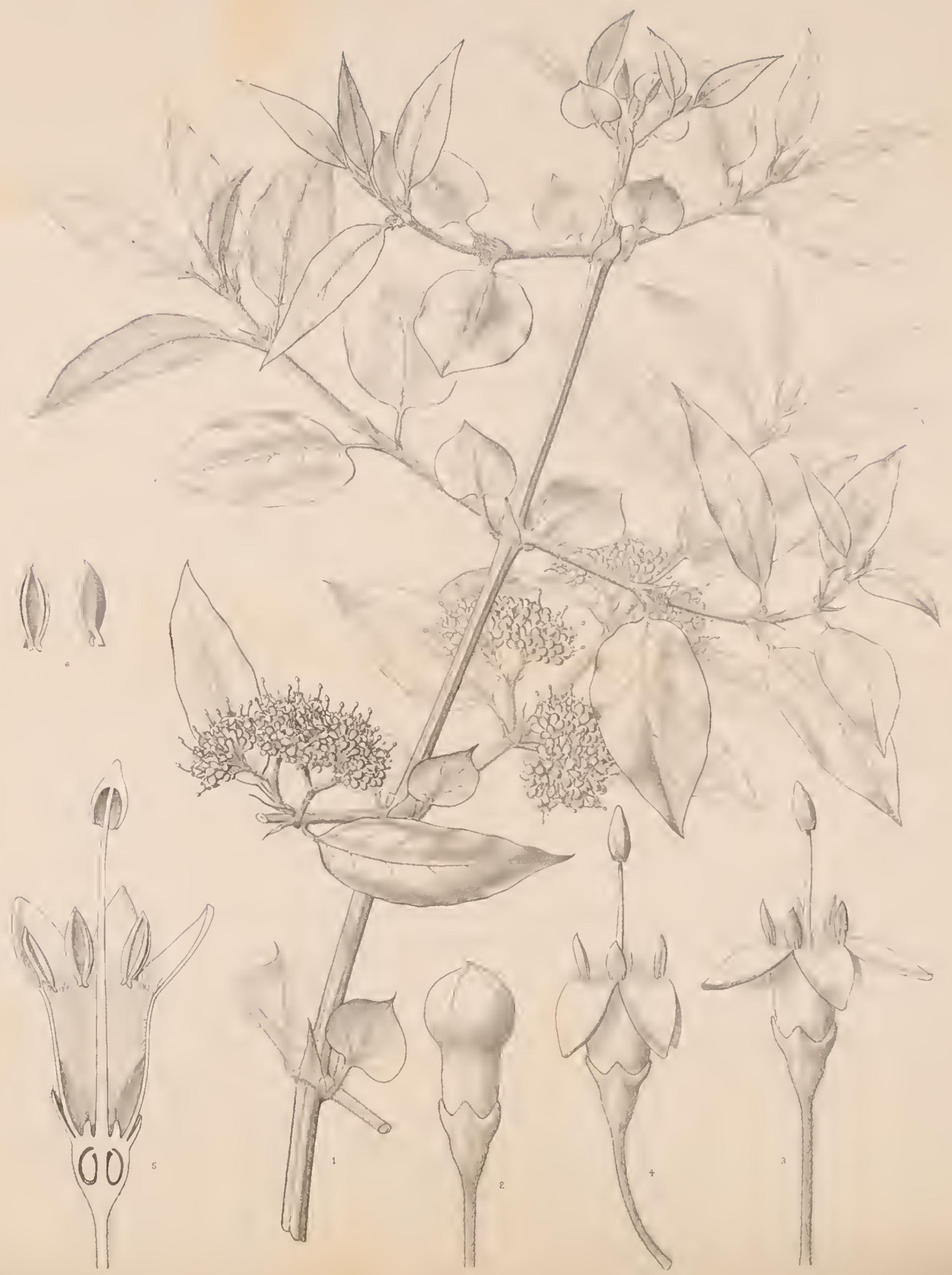




\title{
Plectronia stipulata /le IIild. mor. spe.
}

\author{
(RIBINCH.
}

Plectronia stlpulata; scandens, lamis flurentibus gracilibus, subquadrangularibus, velutinis; foliis petiolatis ovato-oblongis, acutis, basi late cuneatis vel subrotundatis supra setulis inspersis, subtus sparse pubescentibus; stipulis e basi lata subulatis velutinis, triangularibus $17 \mathrm{~mm}$. longis; panicula multiflora, axillari puberula, dichotoma; bracteis lineari-subulatis; floribus pedicellatis, pentameris ; ovario subgloboso, extus velutiıo; calyce fere ad medio in 5 lobos triangulares subacutos, ciliatos diviso; corolla fere ad medium in lobos extus glabros divisa, intus annulo piloso munita; stilo glabro, stigmate ovoideo.

Plante grimpante, à rameaux subquadrangulaires, velus à l'état jeune, devenant plus ou moins glabres avec l'âgre. Fenilles des rameaux principaux et des rameaux secondaires très différentes; feuilles des rameaux principaux largement ovales suhcordées, longuement pétiolées, ì pétiole de $18 \mathrm{~mm}$. de long, canaliculé, velu, à poils brunàtres, à limbe atteignant $25 \mathrm{~mm}$. de long et $30 \mathrm{~mm}$. de large, acuminé, aigu au sommet, glabrescent sur la face supérieure, velu sur la face inférieure, au moins sur les nervures; feuilles des rameaux florifères pétiolées, à pétiole atteignant $12 \mathrm{~mm}$. de long, courtement pubescent, à limbe

Explication des figures de la planche XXXVIII.

Fis. 1. - Fragment de rameau avec inflorescence (1/1).

Fio. 2. - Bouton floral (10/1). 
ovale-oblong, acuminé-aigu, largement cunéiforme à la base ou subarrondi, à soies courtes et éparses sur la face supérieure plus ou moins luisante, éparsement velu sur la face inférieure, au moins sur les nervures, de $5-8 \mathrm{~cm}$. de long et $18-35 \mathrm{~mm}$. de large; stipules largement triangulaires subulées, velues sur le dos, aussi longues ou plus longues que le pétiole des feuilles voisines, atteignant environ $12 \mathrm{~mm}$. de long. Panicules florales axillaires, multiflores, dichotomes, pubescentes-brunâtres, à bractées linéaires-sululées; fleurs pentamères courtement pédicellées, à pédicelle grêle, velu, de $2 \mathrm{~mm}$. environ de long, ‘i ovaire subglobuleux, éparsement velu; à calice environ aussi long que l'ovaire, divisé en 5 lobes triangulaires subaigus, ciliés au sommet. Corolle de 4 à $5 \mathrm{~mm}$. de long divisée jusque vers le milieu en 5 lobes, à tube corollin glabre sur la face extérieure, velu sur la face interne dans sa moitié supérieure, à poils réfléchis, à lobes triangulaires subaigus, glabres sur les deux faces, sauf à la gorge de la corolle où il existe des touffes de poils. Étamines oblonguesovales, courtement appendiculées à la base. Ovaire à disque glabre, à style glabre, à stigmate calyptriforme, ovoïde.

Hab. - Mozambique, forêts du Morrumbala, 15 juin 1901 (Éd. Luja, n. 430 .

Observations. - Le genre Plectronia que les auteurs de la Flora of tropical Africa n'araient pas admis, renfer'me un nombre considérable d'espèces et ce nombre croit journellement, peut-on dire.

Il est trés difficile même, vu le grand nombre d'espèces de ce genre créées l'écemment sur des échiantillons secs, de donner les affinités de la plante que nous figurons ici. Elle appartient au groupe des Plectronia glabrescents, sauf sur les inflorescences, à corolle tétramèrc glabre extérieurement, velue à la gorge. Comme chez un certain nombre d'autres

Fıg, 3 et 4 - Fleurs épanouies ( $/ / 1)$.

Fia. 5. - Coupe longitudinale de la fleur épanouie (10/1).

Fig. 6. - Etamines vues de face et de dos (12/1). 
espèces de co genre, les feuilles de notre plante prennent, en so desséehant. une couleur trouge-lunatio nu'olles conservent; elles ne deviement pas noires comme ehez quelques espèees du nème genre, - earaetère partagé par des espèces du même genre et par dcs types d'autres genres de la famille de Rubiacées. La diftérence des feuilles de la tige principale et des feuilles des rameaux latéraux, est tris aceusée; eomme lindique la deseription, la forme de ces feuilles beaucoup plus longuement pétiolées est largement ovale, cordée, acuminée, tandis que les feuilles raméales sont ovales-oblongues, alr'ondies ou à peine subeordées. P'ar'mi les caractères sur lesquels nous insisterons eneore, eitons les stipules allongées-aiguës, variant en dimension, mais égalant ou dépassant même généralement les pétioles des feuilles entre lesquels elles se développent. Le calice est également assez earaetéristique, il est velu dans sa partie inferieure, glabre dans sa partie supérieure, sauf ver's le sommet des lohes qui portent quelques poils eourts; le style, longuement exsert, est en eapuchon, terminé en pointe obtuse. 


Pl. Noval HERB Hort Thenensis

PL XXXIX

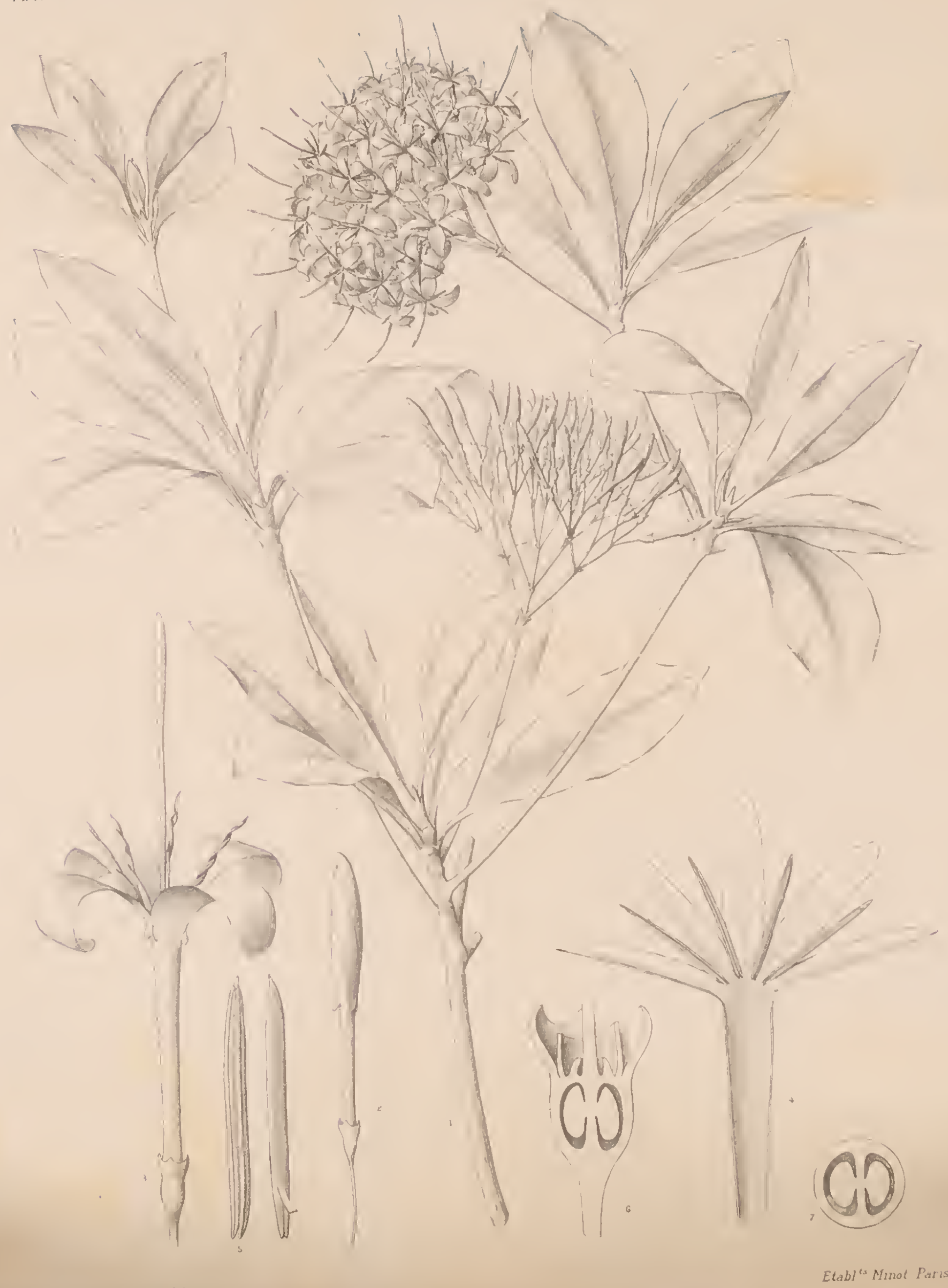




\title{
Pavetta appendiculata De Wild. nov. sp.
}

\author{
(RUBIACE. F)
}

Pavetta appendiculata; pl. ramulis glabris; foliis obovatooblongis, subacutis basi sensim angustatis oblongo-lanceolatis et brevissime petiolatis, $5-10 \mathrm{~cm}$. longis et $1-4 \mathrm{~cm}$. latis; stipulis latis breviter acutis, margine breviter ciliatis ; floribus albidis cymas axillares laxis congestas efformantibus; calycis turbinati limbo 4-dentato, circ. $45 \mathrm{~mm}$. longis: corollae limbi laciniis ovato-oblongis, obtusis, $\$-9 \mathrm{~mm}$. longis, tubum subaeruentibus; stigmate staminibusque exsertis.

Plante buissonnante ramifiée, atteignant 2 à $3 \mathrm{~m}$. de haut, à rameaux cylindriques, glabres. Feuilles disposées vers l'extrémité des rameaux, obovales-oblongues, subaiguës au sommet, rétrécies à la base ou oblongues-lancéolées, très courtement pétiolées, de 5 à $10 \mathrm{~cm}$. de long et de l à $4 \mathrm{~cm}$. de large, pétiole compris, glabres sur la face supérieure, plus pâles en dessous qu'au-dessus, éparsement velues sur les nervures de la face inférieure, nervures latérales au nombre de 8 à 9 de chaque côté de la nervure médiane. Stipules largement triangulaires-aiguës, courte-

\section{Explication des figures de la planche XXXIX.}

FIG. 1. - Fragment de rameau florifère avec inflorescence terminant des rameaux latéraux courts, dont les feuilles supérieures tombées, font paraitre les intlorescences longuement pèdonculèes (1/1).

Fro. 2. - Bouton, les oreillettes de la base des lobes libres de la corolle se remarquent très bien $(4 / 1)$.

Fro 3. - Fleur épanouie (4/1). 
ment ciliées sur le hord. Fleurs blanches, réunies en cymes lâches plusieurs fois dichotomes, sessiles, dressées ou étalées, atteignant $5-6 \mathrm{~cm}$. de long et jusque $8-9 \mathrm{~mm}$. de large, axillaires ou terminant des raneaux latéraux dont les feuilles sont tombées. Calice tétramère, de $2,5 \mathrm{~mm}$. en viron de long, glabre, à 4 dents triangulaires-subaiguës. Corolle glałre extérieurement, à tuhe de 9-12 nmm. environ de long, velu extérieurement, à 4 lobes environ aussi longs que le tube ou un peu plus courts, de 8 à $10 \mathrm{~mm}$. ovales-oblongs, ol,tus, munis à la base, unilatéralement, d'une sorte d'oreillette. Anthères exsertes, un peu plus comrtes que les lobes de la corolle, spiralées après l'anthèse, très courtement stipitées, aiguës au sommet. Ovilire biloculaire, à loges uniovulées, à disque glabre, à style exsert, glabre, fusiforme au sommet, entier, dépassant la gorge de la corolle de $13 \mathrm{~mm}$. environ. Fruits inconnus.

Hab. - Mozambique, Morrumbala, 1900, à 1.200 métres d'altitude (Éd. Luja, n. 376).

Observations. - La plante que nous figurons ici et qui provient de la zone supérieure du Morrumbala, constitue un arbuste de la brousse dont le port et les caractères rappellent le Pavetta gardenicofolia Hochsetter, caractéristique, semble-t-il, pour la zone nilienne (cf. Richard $\mathrm{Fl}$. Abyss. I p. 35 l et $F l$. trop. Afr. IIl p. 176).

Si l'on compare la plante qui nous a été envoyée par M. Éd. Luja à celles r'écoltées par Quartin-Dillon en Abyssinie (env. de Massoua) et par Schweinfurth et Riva dans les environs de Ghinda, on remarque certains détails différentiels qui nous ont porté à créer une espèce nourelle. On ne trouve pas en effet dans les échantillons d'Abyssinie les oreillettes caractéristiques des lobes libres de la corolle, oreillettes qui sont très visibles déjà dans le bouton, comme le fait ressortir en particulier la figure ? de notre planche, qui reproduit un bouton peu avant l'èpanouis-

Fig. 4. - Corolle fendue et étalée (5/1).

FıG. 5. - Étamines vues de face et de dos (10/1).

F1o. f. - Coupe longitudinale de l'ovaire surmonté du disque (14/1).

Fig. 7. - Coupe transversale de l'ovaire (15/1). 
sement et sous un grossissement de 4 diamètres. Les autres caractères : villosité légèrement plus accusée sur les feuilles jeunes dans la plante du Mozambique, plus accusée à l'intérieur des stipules dans la plante abyssinienne, sont des caractères de plus ou de moins, difficiles à apprécier dans une diagnose et qui se trouvent indiscutablement sous l'action des agents exterieurs; aussi ne voulons-nous pas insister' sur eux. Il en est de même du caractère tiré de la disposition des inflorescences : dressées ou recourbécs; ces deux dispositions se remarquent souvent sur la méme tige, elles n'ont donc à notre avis aucune valeur'.

Notre plante se rapproche davantage du $P$.gardenicefolia recueilli par (Wuartin-Iillon que de celui recueilli jar le I ${ }^{\mathrm{r}}$ Schweinfurth et dont nous n'avons, il est vrai, vu que des rameaux fructiferes; mais tandis que chez ce dernier les feuilles sont r'estées d'un vert pâle, mat à l'état sec, chez la plante d'Abyssinie comme chez celle du Mozambique les feuilles ont bruni. la face supérieure étant nettement plus foncée que la face inférieur'e. Nous n'avons pas à discuter ici les différences dans deux formes abyssiniennes; le type tel qu'il est compris dans la Flora of tropical Africa III p. 17 renfel merait d'ailleur's plusieur's variétés : longiflora Vatke, breviflora Vatlse, à fleul's plus ou moins allongées. Sans insister, nous pourrions ajouter que dans la longueur des parties de la fleur il existe encore un caractère différentiel de notre Pavetta appendiculata, les fleur's du typique $P$. gardeniafolia Hoclsst. mesurant de 6 à $9 \mathrm{~mm}$. le long seulement, à lobes environ aussi longs que le tube. Dans la variété longiflora la fleur atteint $2,8 \mathrm{~cm}$. de long, dans la variété breviflora elle mesure environ $9 \mathrm{~mm}$. de long. 



$$
\text { - }
$$

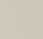





\section{PLANT $\nRightarrow$ NOV E}

VEL MINUS COGNITE

EX IIERBARIO HORTI THENENSIS

\section{PLANTES NOUVELLES OU PEU CONNUES}

CONTHNUES DANS L'HERBIER

DE

L'HORTUS THENENSIS

AVEC LES DESCRIPTIONS OU ANNOTATIONS

DE

\section{M Ém. DE WILDEMAN}

DOCTEUR EN SCIENCES

CONSERVATEUR AU JARDIN BOTANIQUE DE L'ÉtAT

Gixième livraison.

(JUILLET 1906)

VEUVE MONNOM

IMPRIMFUR FT KDITHUR

32, RUE DE L'INDUSTRIE, 32

BRUNELLES

Se vend exclusivement à l'Administration des biens et affaires de M. van den Bossche, 5, Grande Montagne, à Tirlemont. 



\section{SIXIEME LIVRAISON}

Cette nouvelle livraison des Planta Nova devant, dans mes intentions, être consacrée à la flore sud-africaine, j'ai voulu y faire figurer un certain nombre d'espèces qui, pour la plupart, ont été créées an cours des dernières années et qui, par conséquent, n'ont pas encore été analysées dans d'autres recueils.

Les unes se trouvent dans les intéressants matériaux d'herbier que M. Harry Bolus a bien voulu m'offrir très gracieusement, - et, pour lui en exprimer toute ma reconnaissance, je ne pouvais mieux faire que d'en publier dans ce recueil quelques croquis et analyses. Quelques autres ont été choisies dans la collection de M. Rud. Schlechter, ì raison de leur affinité avec les premières.

Les espèces figurées sont au nombre de quatorze, it savoir :

Lotononis decipiens $R$. Schl.

Lotononis arenicola $R$. Schl.

Lotononis delicatula Bolus.

Lotononis Maximiliani $R$. Schl.

Aspalathus cliffortioides Bolus.

Aspalathus leptoptera Bolus.

Aspalathus Schlechteri Bolus.

Phyllosma capensis Bolus.

Muraltia spicata Bolus.

Gnidia pulvinata Bolus.

Gnidia transvaliensis Gilg.

Gymnostephium læve Bolus.

Phymaspermum appressum Bolus.

Senecio sociorum Bolus. 
Parmi ces espèces, il en est quelques-unes dont nous n'avons pu trouver la description originale dans aucune publication à nous connue et, après avoir été aux informations à Kew comme à Berlin, nous devons supposer que ces descriptions n'ont pas encore été publiées. J'ai donc prié $\mathrm{M}$. le $\mathrm{D}^{\mathrm{r}}$ De Wildeman de faire précéder ses observations d'une diagnose latine, qui, jusqu'à plus ample information et tout en respectant le droit des auteurs, puisse en tenir lieu.

L. B. 


\section{LOTONONIS $D C$.}

Il n'entre pas dans le cadre de cette publication - nous avons déjà eu l'occasion de le dire - d'étudier', comme dans les Icones, la systématique des espèces figurées.

Nous croyons toutefois qu'il est utile, avant tout, de rappeler les caractères les plus importants du groupement des Crotalariinece, dans la tribu des Genistec-Leguminos $\propto$, puisque nous avons à étudier successivement quelques espèces de Lotononis et d'Aspalathus, deux genres qui, l'un et l'autre, comptent un très grand nombre de représentants dans la flore sud-Africaine - tout comme les genres voisins au nombre de onze qui, eux, sont exclusivement confinés dans cette région.

Les dits genres sont tous caractérisés par la glabréité du style et par l'absence de stipules libres. Les uns (Borbonia, Rafnia, Euchlora) ont les feuilles simples et planes; les autres possèdent le plus souvent des feuilles à trois folioles, parfois réduites à un seul foliole, mais toujours cylindriques et filamenteuses. Les Lotononis se différencient de tous autres parce qu'ils ont les quatre lobes supérieurs du calice soudés deux ì deux, le lobe inférieur restant libre. Les Aspalathws, au contraire, ont les cinq lobes du calice à peu près égaux ct ils partagent ce caractère avec les Lebeckea; mais ils se distinguent de ce dernier genre, à feuilles pétiolées, par leurs feuilles et folioles sessiles.

M. Rud. Schlechter, qui est un des botanistes européens ayant le plus étudié la distribution des végétaux dans le sud de l'Afrique, a donné au sujet de ce genre quelques renseignements intéressants dans les Jahrbücher du Prof. Ad. Engler (Vol. XXVII, 1899, p. 147). Il insiste dans ces notes sur l'état précaire de nos connaissances quant à la valeur des sous-genres ou sections et propose deux groupements basés sur l'habitat et la forme des différentes espèces, à savoir' : les formes descendantes et annuelles dont les centres de développement seraient dans la région du Namaqualand et les espèces dressées ou sous-buissonnantes qui sembleraient aroir leur centre dans la région orientale. C'est dans le Namaqualand que se rencontrent les espèces à fleurs aux couleurs vives, particulièrement jaunes, parfois rouges, très rarement bleues; les espèces à fleurs de cette dernière couleur sont toutes de la 
côte sud. Les plantes sur lesquelles nous attirons ici l'attention sont toutes à fleurs jaunes.

Harvey et Sonder, dans la Flora capensis (II, p. 48), ont donné le tableau des groupements qui avaient été effectués dans le genre à l'époque de la publication de leur ouvrage et qui ont été repris dans les Pflanzenfamilien. Ils sont au nombre de huit et peuvent se caractériser par la disposition des fleurs, en cymes, en grappes, en capitule ou solitaires. - Toutes les formes que nous avons fait figurer ici, afin de donner quelques matériaux de comparaison à ceux qui voudraient entamer l'étude monographique de ce genre, possèdent des fleurs solitaires. Elles se rangent donc d'après ce caractère, dans l'une des quatre sections: Telina Harv., Krebsia Eck. et Zeyher, Leobordca Delile et Leptis Benth. Mais si l'on étudie plus en détail leurs caractères d'après la clef proposée par'Taubert, on verra que les deux premières subdivisions, qui renferment également des espèces ì fleurs disposées en grappes, ne peuvent entrer en ligne de compte et que, des deux dernières, la section Leptis Benth., caractérisée par la petitesse du lobe inférieur de son calice, est également à écarter. Les plantes que nous figurons appartiendraient dès lors toutes à la section Leobordea. Mais comme le fait ressortir M. Schlechter Lcobordea et Leptis sont peu différents. 


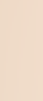


D. NOVAE HFri HORT THEnensis

Pl. XI.
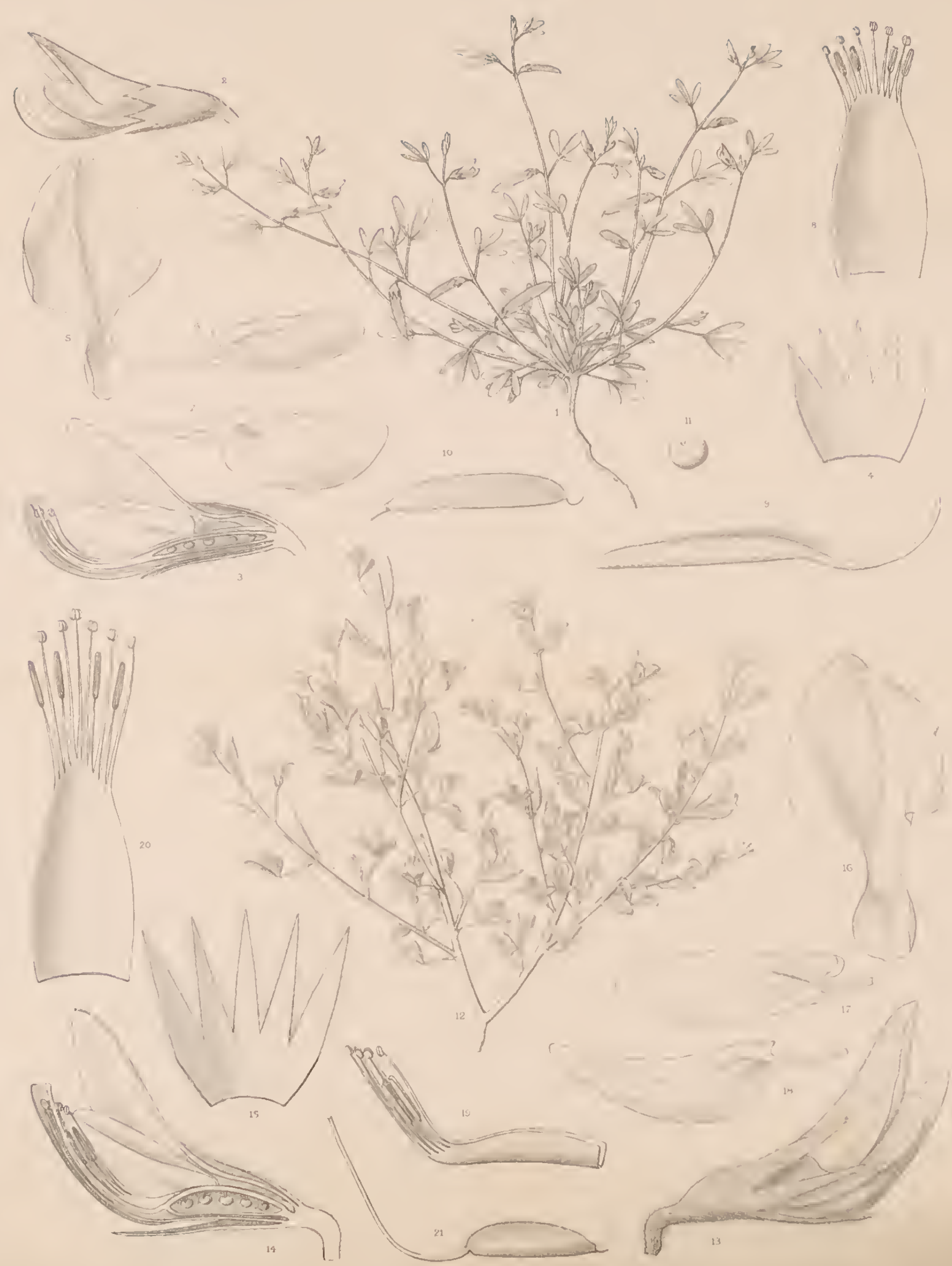


\section{Lotononis decipiens R. Schlechter nor. sp.}

Lotononis decipiens; herba pusilla, decumbens vel ascendens, e basi ramosa; ramis teretibus, tenuiter strigosis, foliatis ; foliis trifoliolatis petiolatis, petiolo strigoso, foliolis obovatis vel oblongis, sparse strigosis, $5-10 \mathrm{~mm}$. longis et supra medium circ. $1,53 \mathrm{~mm}$. latis, foliolis lateralibus subrquilongis; stipulis. . . . ; pedunculis suberectis, circ. $1 \mathrm{~mm}$. longis, oppositifoliis, folia multo minoribus, strigosis, calyce brevioribus; bracteis circ. $1 \mathrm{~mm}$. longis; calyce $3 \mathrm{~mm}$. circ. longo, strigilloso usque medio 5-fisso, segmentis lanceolatis acutis subrequilongis; vexillo unguiculato, lamina orbiculari, apice rotundata, circ. $6 \mathrm{~mm}$. longa et medio fere $2,5 \mathrm{~mm}$. lata, dorso glabra; alis graciliter unguiculatis, lamina obovata-oblonga, obtusissima, glaberrimis, circ. $4 \mathrm{~mm}$. longis et $1 \mathrm{~mm}$. latis; carina subobtusa, glaberrima, unguiculata, circ. $5 \mathrm{~mm}$. longa; stylo lineari, incurvo, glabro; ovario puberulo, 10 ovulato; legumine $15 \mathrm{~mm}$. longo velutino.

Petite plante herbacée, ¿̀ rameaux ascendants ou couchés, cylindriques, courtement velus, à feuilles épurses, trifoliolées, pétiolées, ¿ pétiole strigilleux, à folioles ubovales ou oblongues, éparsement velues, de $\check{5}-10 \mathrm{~mm}$. de long et de 1,5-3 mm. de large. Folioles latérales environ aussi longues que la terminale; stipules caduques. Pédoncule

\section{Explication des figures 1-11 de la planche XL.}

Lotononis decipiens $R$. Schlechter.

FIG. 1. - Plante fleurie (1/1).

Fia. 2. - Fleur épanouie (6/1).

Fio. 3. - Coupe longitudinale de la fleur $(9 / 1)$.

Fia. 4. - Calice fendu et étalé $(9 / 1)$. 
subérigé ou étalé, d'environ $1 \mathrm{~mm}$. de long, opposé aux feuilles et beaucoup plus court qu'elles, velu, plus court que le calice. Celui-ci de $3 \mathrm{~mm}$. environ de long, strigilleux, quinquefide, à segments lancéolés, aigus, de même longueur, environ aussi longs que le tube. Étendard onguiculé, à onglet élargi à la base, à lame arrondie au sommet, de $2,5 \mathrm{~mm}$. environ de large, glabre sur le dos; ailes à onglet grêle, à lame obovale-oblongue, obtuse, glabre, de $4 \mathrm{~mm}$. environ de long; carène subobtuse, onguiculée, d'environ $5 \mathrm{~mm}$. de long. Ovaire courtement velu, sauf sur la crète supérieure munie de poils plus allongé, ¿̀ environ 10 ovules; style linéaire recourbé, glabre. Légume de $15 \mathrm{~mm}$. environ de long, velu, terminé par le style assez longtemps persistant.

Hab. - Région austro-occidentale [Brackdam in collibus, 1,800 ped. ( $(7$ avril 1897), leg. Rud. Schlechter (Herb. n. 11,1 13)].

\section{Lotononis arenicola $R$. Schlechter nov. sp.}

Lotononis arenicola; herba suffruticosa, decumbens vel ascendens e basi ramosa; ramis teretibus, argenteo-strigosis, foliatis; foliis 3 -foliolatis, longe petiolatis, petiolo argenteo-strigoso, circ. 6-7 mm. longo; foliolis obovatis, subemarginatis, $5-7 \mathrm{~mm}$. longis et 3-4 mm. latis, supra et infra dense argenteo-villosis; stipulis oblique ovatis, argenteo-villosis, $3 \mathrm{~mm}$. circ. longis, acutis; pedunculis suberectis, circ. $3-3 \mathrm{~mm}$. longis, oppositifoliis, argentatis, calyce bre-

\section{Lotononis decipiens $R$. Schlechter (suite).}

Fıg. 5 - Éteudard, vu par la face interne (9/1).

Fı. 6 - Aile $(9 / 1)$.

FIG. 7. - Garène (9/1).

FIG. 8. - Androcée étalé (9/1).

H'r.s. 9. - Ovaire et style (13/1).

FIG. 10. - Légume mûr (3/1).

FIa. 11. - Graine grossie. 
vioribus; calyce 7-8 mm. circ. longo, strigilloso, ultra medio 5 fisso, segmentis lanceolatis, acutis, subrequilongis, $4-5 \mathrm{~mm}$. longis; vexillo unguiculato, lamina orbiculari, apice rotundata, circ. $11 \mathrm{~mm}$. longa et medio fere (i mm. lata, dor'so dense velutina; alis graciliter unguiculatis, lamina obovata-oblonga, obtusissima, glaberrinis, circ. $9 \mathrm{~mm}$. longis; carina subacuta, glaberrima, alis longiora, apice ascendente, circ. $10 \mathrm{~mm}$. longa; stylo lineari, basi incurvo, glabro; ovario puberulo 10 ovulato.

Plante herbacée ou suffrutescente, à rameaux ascendants ou couchés, cylindriques, à indument argenté. Feuilles trifoliolées, assez longuement pétiolées, à pétiole velu-argenté, de 6 à $7 \mathrm{~mm}$. de long, à folioles obovales, subémarginées au sommet, de 5 à $7 \mathrm{~mm}$. de long et de 3 à $4 \mathrm{~mm}$. de large, densément velues argentées sur les deux laces; stipules obliquement ovales, argentées-villeuses, de $3 \mathrm{~mm}$. environ de long, aiguës. Pédoncule étalé ou dressé de 2 à $3 \mathrm{~mm}$. de long, opposé aux feuilles, veluargenté, plus court que le calice. Celui-ci de 7 à $8 \mathrm{~mm}$. environ de long, strigilleux, fendu en 5 lobes jusqu'au delà du milieu, segments subégaux, lancéolés, aigus de 4 à $5 \mathrm{~mm}$. environ de long. Étendard onguiculé, à onglet élargi à la base, à lame orbiculaire, arrondie au sommet, de $6 \mathrm{~mm}$. environ de large, glabre sur la face interne,

\section{Explication des figures $12-21$ de la planche XL.}

Lotononis arenicola $R$. Schlechter.

Frs. 12. - Fragment de la plante fleurie (1/1).

Fia 13. - Fleur épanouie (5/1).

FIG. 14. - Coupe longitudinale de la fleur (5/1).

Fıg. 15. - Calice fendu et étalé $(5 / 1)$.

FIa. 16. - Étendard (5/1).

Fig. 17. - Aile (5/1).

Fia. 18. - Carène (5/1).

Frg. 19. - Androcée, vu de profil (5/1).

Fır. 20. - Androcée étalé (6/1).

FIG. 21. - Ovaire et style (5/1). 
densément velue sur la face externe; ailes onguiculées, à lame obovale-oblongue, obtuse, glabre, plus courtes que l'étendarıl; carène subaiguë, glabre, un peu plus longue ¡ue les ailes, de $10 \mathrm{~mm}$. environ de long. Ovaire velu dans sa partie supérieure, à 10 orules; style linéaire, plus long que lovaire et assez fortement recourbé.

Hab. - Région austro-occidentale [Taus, in collibus 2,300 ped. (12 avril 1897), leg. Rud. Schlechter (n. 11,206)].

Observations. - Les deux espèces dont nous venons de domner ciavant la description détaillée et dont les figures se trouvent reproduites dans la planche XI, appartiennent toutes deux au même groupe. Plusieurs parties de leurs fleurs sont de forme semblable; le calice est à dents égales, plus longues chez le L.arenicola que chez le $L$. decipiens; l'étendard a la même forme chez les deux espèces : glabre chez le $L$. decipiens, il est velu sur le dos chez le L. arenicola; il y a une légère différence dans la forme des ailes et dans celle de la carène. Quant à l'androcée, les filaments libres sont plus allongés clie $L$ le $L$. arenicola qu'ils ne le sont chez le $L$. decipiens.

Le $L$. decipiens est une forme plus grêle et moins ramifiée que le $L$. arenicola et dans la forme de folioles, nous trouvons un caractère permettant une différenciation facile; en effet, chez la dernière de ces espèces les folioles sont oborales, émarginées au sommet, tandis qu'elles sont oblongues chez la première; le rapport entre la longueur et la largeur des folioles est représenté par un chiffre plus élevé chez le L. decipiens que chez le $L$. arenicola. 

Pi Novae llerb Hort. Thenensis.

$P_{L} \times 1.1$.

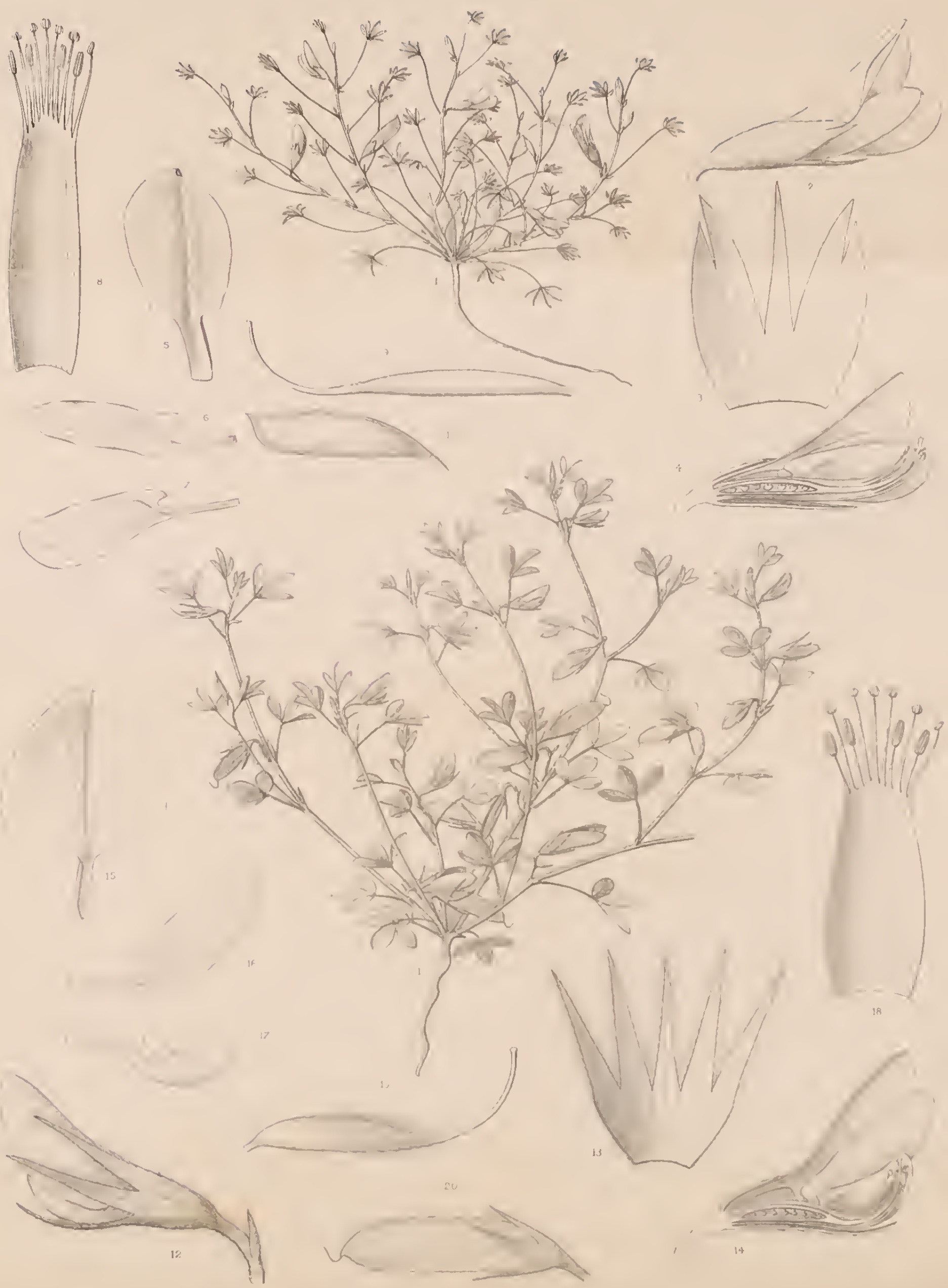




\section{Lotononis delicatula Bolus nov. sp.}

Lotononis delicatula; herba pusilla, dccumbens c basi ramosa, ramis terctibus, sparse pilosis, foliatis; foliis 3-5-foliolatis, louge et graciliter petiolatis, pctiolo sparse piloso, 10-20 mm. longo, foliolis oblongis vel obovato-oblongis, subacutis, $3-5 \mathrm{~mm}$. longis et 0,7-15 mm. latis, sparse pilosis et ciliatis; stipulis angustis, caducis ; pedunculis suberectis, circ. I mm. longis, oppositifoliis, calycc brevioribus; calyce $5 \mathrm{~mm}$. circ. longo, sparse strigilloso, 5-fisso, segmentis lanccolatis, antice acutioribus; vexilla unguiculata, lamina suborbiculari, apice rotundata, circ. $10 \mathrm{~mm}$. longa ct $4 \mathrm{~mm}$. lata, glabra; alis graciliter unguiculatis, lamina obovata-oblonga, obtusa, glaberrimis, circ. $10 \mathrm{~mm}$. longis; carina obtusa, glaberrima alis subæquilonga; stylo lineari, glabıo; ovario margine superiorc puberulo, 10-15 ovulato; lecrumine subglabrescens, circ. 12 mrn. longo ct $3 \mathrm{~mm}$. lato.

Petite plante grêle, décombante, rameuse à la base, à rameaux cylindrinues, éparsement velus et feuillus Feuilles à 3 ou 5 folioles, longuement pétiolées, à pétiole garêle,

Explication des figures 1 - 10 de la planche XLI.

Lotononis delicatula Bolus.

Fıo. 1. - Plante entière fleurie (1/1).

Fis. 2. - Fleur épanauie (5/1).

frı. 3 - Calice fendu et étalé (14/1).

Fia. 4. - Corolle fendue (5/1).

Fig. 5. - Étendard (5/1).

Fro. 6. - Aile (5/1).

Fı. 7 - Carène (5/1).

Fia. 8. - Androcée ètalé (7/1).

Fso. 9. - Ovaire et style (9/1).

Fı́. 10. - Légume, mủr (4/1). 
éparsement velu, de 10 à $20 \mathrm{~mm}$. de long; folioles oblongues ou obovales-oblongues, subaiguës, de $3-5 \mathrm{~mm}$. de long et de 0,7-1,5 mm. de large, éparsement velues et ciliées; stipules étroites et assez rapidement caduques. Pédoncule subérigé, d'environ $1 \mathrm{~mm}$. de long, solitaire, opposé aux feuilles, plus court que le calice, celui-ci de $5 \mathrm{~mm}$. environ de long, éparsement strigilleux, quinquefide, à segments de longueur inégale, l'antérieur environ 2 fois aussi long, dans sa partie libre, que les autres segments. Étendard de $10 \mathrm{~mm}$. environ de long et de $4 \mathrm{~mm}$. environ de large, glabre, onguiculé, à lame suborbiculaire environ 2 fois aussi longue que l'onglet, arrondie au sommet; ailes onguiculées, à onglet grêle, à lame obovaleoblongue, obtuse, glabre, de $10 \mathrm{~mm}$. environ de long; carène oltuse, glabre, environ aussi longue que les ailes, Ovaire pubéruleux sur le bord supérieur, à 10-15 ovules; légume subglabrescent de $12 \mathrm{~mm}$. environ de long et de $3 \mathrm{~mm}$. environ de large.

Hab. - Région austro-occidentale [Brackdam in collibus, 1,800 ped. (7 avril 1897) leg. Rud. Schlechter (n. 11,106)].

\section{Lotononis Maximiliani $R$. Schlechter nor. sp.}

Lotononis Maximiliani herba pusilla, decumbens, e basi ramosa; ramis teretibus, plus minus dense strigillosis, foliatis; foliis 3 -foliolatis, longe petiolatis, petiolo strigilloso, 8-12 mm. longo, foliolis oblongis vel obovato-oblongis, subacutis, $6-10 \mathrm{~mm}$. longis et $2-4 \mathrm{~mm}$. latis, supra et infra dense pilosis; stipulis oblique-ovatis, velutinis et ciliatis, acutis, $4 \mathrm{~mm}$. longis, caducis; pedunculis apice recurvatis rel suberectis 4-6 mm. longis, oppositifoliis versus medium articulatis et bracteatis, calyce brevioribus rel subæquantibus, post anthesim accrescentibus; calyce $8 \mathrm{~mm}$. circ. longo, strigilloso-ciliato, profunde 5-fisso, segmentis lanceolatis tubo longioribus; vexillo circ. $11 \mathrm{~mm}$. longo et $6 \mathrm{~mm}$. lato, anguste unguiculato, lamina suborbiculari, apice rotundata, glabra; alis graciliter unguiculatis, lamina obovata-oblonga, obtusa, glaberrima, circ. $9 \mathrm{~mm}$. longis ; carina subobtusa, glaberrima, alis breviore; stylo lineari, glabro, ovario villoso, 10-15 ovulato; legumine spar'se villoso, $15 \mathrm{~mm}$. longo, $5 \mathrm{~mm}$. lato, stylo coronato. 
Plante herbacée, à rameanx décombants, cylindrirues plus on moins densément strigilleux, fenillus. Feuilles trifoliolées, assez lolignement pétiolées, à pétiole strigilleux de 8-12 min. de long. Folioles oblong̨ues ou obovales-ohlongutes, subriguës, de 6-10 mm. de long et 2-4 mun. de liırge, assez densément velues sur les deux faces; stipules obliquement ovales, velues et cilices, aignës, de 4 mm. environ de long, caduques. Flemr's opposées aux fenilles, à pédoncule recourbé au sommet ou subérigée, de 4-6 $111 \mathrm{~m}$. cuviron de long, anticnlé ver's le milieu et hractéolé, julus court ou environ aussi long que le calice, accrescent aju’ès l'anthèse. Calice de $8 \mathrm{~mm}$. environ de long, strigilleux-cilié, profondément quinquefide, à segments lancéolés, plus long „ue le tube. Étendirrl étroitement onguiculé, de $11 \mathrm{~mm}$. environ de long et de $6 \mathrm{~mm}$. de large, à lanne suborbiculaire arrondie an sommet, glabre; ailes onguiculées, à lante obovale-ohlongue, obtuse, glabre, de $9 \mathrm{~mm}$. environ de long; carène subohtuse, glabre, plus courte que les ailes et que l'étendard. Ovaire velu daus les deux tier's supérieur's, à 10-15 ovules, à style linéaire recourbé, glabre. Légume épar'sement velu dẹ $15 \mathrm{~mm}$, environ de long', couronné par le style persistant.

\section{Explication des figures 11-20 de la planche XLI.}

Lotononis Maximiliani $R$. Schlechter.

Fig. 11. - Plante fleurie (1/1).

Fig. 12. - Fleur épanouie (1/5).

Fır. 13. - Calice fendu et étalé $(5 / 1)$.

Fig. 14. - Corolle en coupe longitudinale (5/1).

Fig. 15. - Étendard (5/1).

Fro. 16. - Aile (5/1).

Fig. 17. - Carène (5/1).

FIG. 18. - Androcée étalé (7/1).

Fig. 19. - Ovaire et style (7/1).

Fra. 20. - Lègume dans le calice (3/1). 
Hab. - Région austro-occidentale, [Achtertuin in collibus 800 ped. (15 août 1897), leg. Rud. Schlechter (n. 10,851)].

Observations. - Des deix plantes que nous avons figurées planche XLI et dont nous venons de donner la description, le L. Maximiliani se rapproche des deux Lotononis que nous avons figurés dans la planche XL; il se rapproche du $L$. arenicola par la forme du calice à dents longues, du $L$. decipiens par son androcée. L'étendard est de constitution très différente et le caractère de cette partie de la fleur rappelle celui que l'on observe chez le $L$. delicatula. Quant à la forme des folioles, elle est intermédiaire entre celles des folioles des deux plantes de la planche XL : plus développécs que celles du $L$. decipiens, parfois même plus larges que celles du $L$. arenicola, elles sont plus nettement obovales que chez la première de ces deux plantes, mais non émarginées au sommet.

Quant au $L$. delicatula, il se différencic aisément des deux plantes de notre planche XL et du L. Maximiliani, tout d'abord par ses feuilles quinquefoliées, caractère assez peu fréquent parmi les espèces du genre Lotononis. Il y a encore dans le calice un caractère très particulier qui écarte cette plante du groupe dans lcquel doivent entrer les trois autres; le calice est fendu en 5 lobes très inégaux, le lobe inférieur étant lancéolé, deux fois aussi long que la partie non divisée du tube calcinal, et les lobes latéraux étant soudés dans les deux tiers inférieurs. 


\section{ASPALATHUS $l$.}

Comme le fait ressortir M. R. Schlechter, dans le second fascicule de ses Ilanta Schlechteriance nova vel minus cognitce, malgr'é le grand nombru des espèces du genre, ces espèces ont une distribution très réduite : chaque colline a sa forme particulière; mais il y a cependant un certain nombre d'espèces qui, dans le domaine de la flore du Cap, sont assez répandues. Le botaniste allemand cite parmi celles-là les $A$. comosa Thunb., lancifolia Berg., spinosa L., vetroflexa L., galioides L., divaricata Thunb.

Certaines les formes du genre telles que les A. lancifolia Berg. et A. spinosa Walp. se rencontrent au Natal; l'A. eriophylla Walp. a été également retrouvé au Natal, près de Grahamstown, et aussi dans la Cafrerie.

Les Aspalathus ne paraissent pas exigents pour le terrain : on en trouve dans toutes les conditions; mais, d'après les constatations de M. R. Schlechter, les variations seraient beaucoup plus nombreuses dans des terrains argileux que dans des sols purement sablonneux. La généralité des Aspalathus possède des fleurs colorées en jaune; quelques espèces ont la corolle blanche, et quelques autres, telle l'A. nigra L., ont des corolles d'un violet foncé.

Nous ne pouvons entrer ici dans le détail des caractères des douze sections qui constituent le genre Aspalathus; il suffira de donner la liste de ces sections et d'ailleurs nous reviendrons sur celles qui nous intéressent à propos des espèces que nous figurons.

$$
\begin{array}{cc}
\text { Section } & \text { I - Cephalanthæ. } \\
" & \text { II - Sericeæ. } \\
" & \text { III - Synpetalæ. } \\
" & \text { IV - Leptanthæ. } \\
" & \text { V - Laterales. } \\
" & \text { VI - Microcarpæ. } \\
" & \text { VII - Grandifloræ. } \\
" & \text { VIII - Pachycarpeæ. } \\
" & \text { IX - Camoræ. }
\end{array}
$$




$$
\begin{array}{cc}
\text { Section } & \mathrm{X}-\text { Pingues. } \\
" & \text { XI }- \text { Terminales. } \\
" & \text { XII }- \text { Pedunculares. }
\end{array}
$$

Les $A$. cliffortioides Bolus, A. leptoptera Bolus, et $A$. Schlechteri Bolus, qui sont figurés ci-après, appartiennent aux deux sections latérales et terminales. 


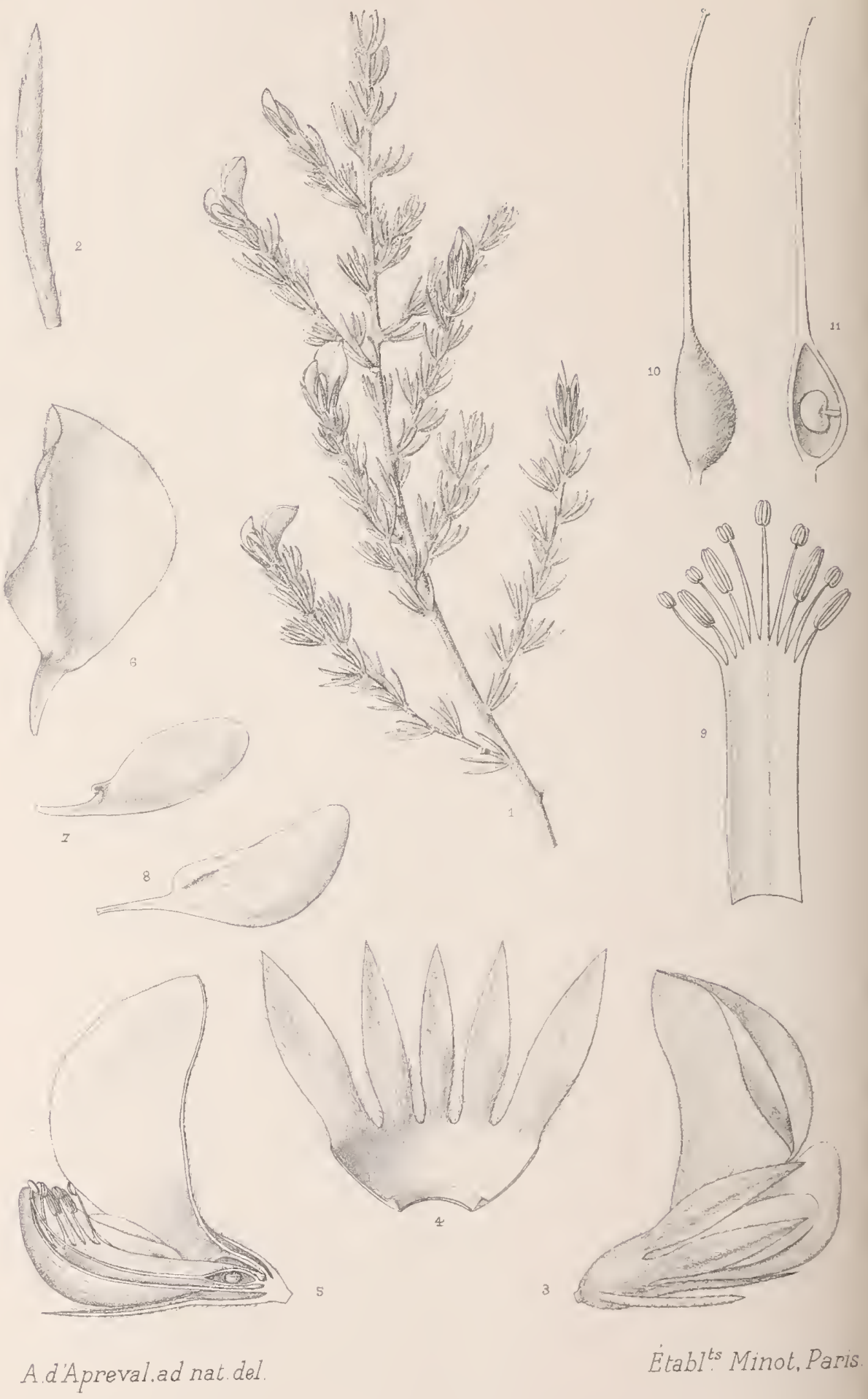




\section{Aspalathus cliffortioides Bo/us}

ex Schlechter in Engler Bot. Jahrb. XXVII (1S99) p. 144.

Petite plante frutescente; rameuse, dressée ; a rameaux ascendants, feuillus, atteignant parfois plus de $25 \mathrm{~cm}$. de long, à ramuscules séricés-pubescents. Feuilles réunies en fascicules étalés, naissant dans une sorte de coussinet villeux, inerme, à limbe linéaire-claviforme, aigu, finement séricé-pubescent. Fleurs terminales, solitaires, sessiles, de I cm. environ de long; bractéoles foliacées, linéaires, plus longues que le tube du calice, mais n'atteignant pas l'extrémité des lobes, ciliées. Calice turbiné de $7 \mathrm{~mm}$. environ de long, à segments lancéolés, aigus, assez épais, trinerviés, deux fois aussi longs que les trois antres. Ftendard suborbiculaire, séricé extérieurement, de $1 \mathrm{~cm}$. environ de long, cilié sur les bords; ailes elliptiques glabres, notablement plus courtes que l'étendard; carène cymbiforme subohtuse, glabre, dépassant les ailes. Faisceau staminal à

\section{E plication des figures de la planche XLII.}

Fig. 1. - Rameau florifère (1/1).

FIG. 2. - Feuille (6/1).

Fı. 3. - Fleur épanouie, vue de profil (4/1).

Fı́. 4. - Calice fendu el étalé $(5 / 1)$.

Fig. 5. - Corolle fendue (4/1).

Fro. 6. - Étendard (4/1).

FIG. 7. - Aile $(4 / 1)$.

Frs. 8. - Carène (4/1). 
4 anthères plus développées que les autres. Ovaire ovoïde, séricé, uni ou biovulé, terminé par un style grêle légèrement capité au sommet.

\section{Hab. - Région austro-occidentale, [Genadendal (R. Schlechter)].}

Observations. - L'A. cliffortioides rappelle par son port un Cliffor. tia et c'est d'ailleurs pour ce fait que M. H. Bolus lui a donné cette dénomination; comme la diagnose et la figure le font suflisamment voir, c'est dans la section des "Terminales " qu"il faudra classer la plante que nous figurons ici d'après un échantillon authentique de M. Schlechter.

Les caractères de cette section sont donnès comme suit par Harvey dans la Flora Capensis II, p. 99 :

Feuilles en touffes, cylindriques ou trigones, rarement solitaires ou ternées, non charnues. Fleurs petites à l'extrémité des raneaux, solitaires, géminées ou disposées en petits racèmes ou épis. Pétales soyeux ou glabres. Calice turbiné ou largement campanulé. Légume obliquement lancéolé, glabre ou soyeux, peu renflé.

Dans le groupement ainsi constitué et dont les caractères peuvent varier dans une assez grande mesure, les espèces peuvent se classer d'après la disposition des feuilles et des fleurs. Par ses feuilles en touffes glabres ou très légèrement soyeuses, ses fleurs solitaires à l'aisselle des feuilles supérieures, l'A cliffurtioides parait devoir se placer dans le voisinage $\operatorname{des} A$. retroflexa L., galioides L., murginalis E. et Z. et exiles Harv. Par ses lobes calicinaux plus longs que le tube et son ovaire velu soyeux la plante décrite par M. H. Bolus doit se ranger, pensons-nous, près de l'A. retroflexa L., dont la description publièe par' la Flora of trop. Africa est relativement vague et ne permet pas, en l'absence de matériaux authentiques de l'espèce, de s'appesantir sur les caractères différentiels. Les notes de M. Schlechter (loc. cit.) ne donnent aucun renseignement précis sur les affinités de la plante que nous figurons.

Fı́. 9. - Androcée étalé (5/1)

Fis. 10. - Ovaire, style et stigmate (10/1).

FIG. 11. - Ovaire en coupe longitudinale (10/1). 


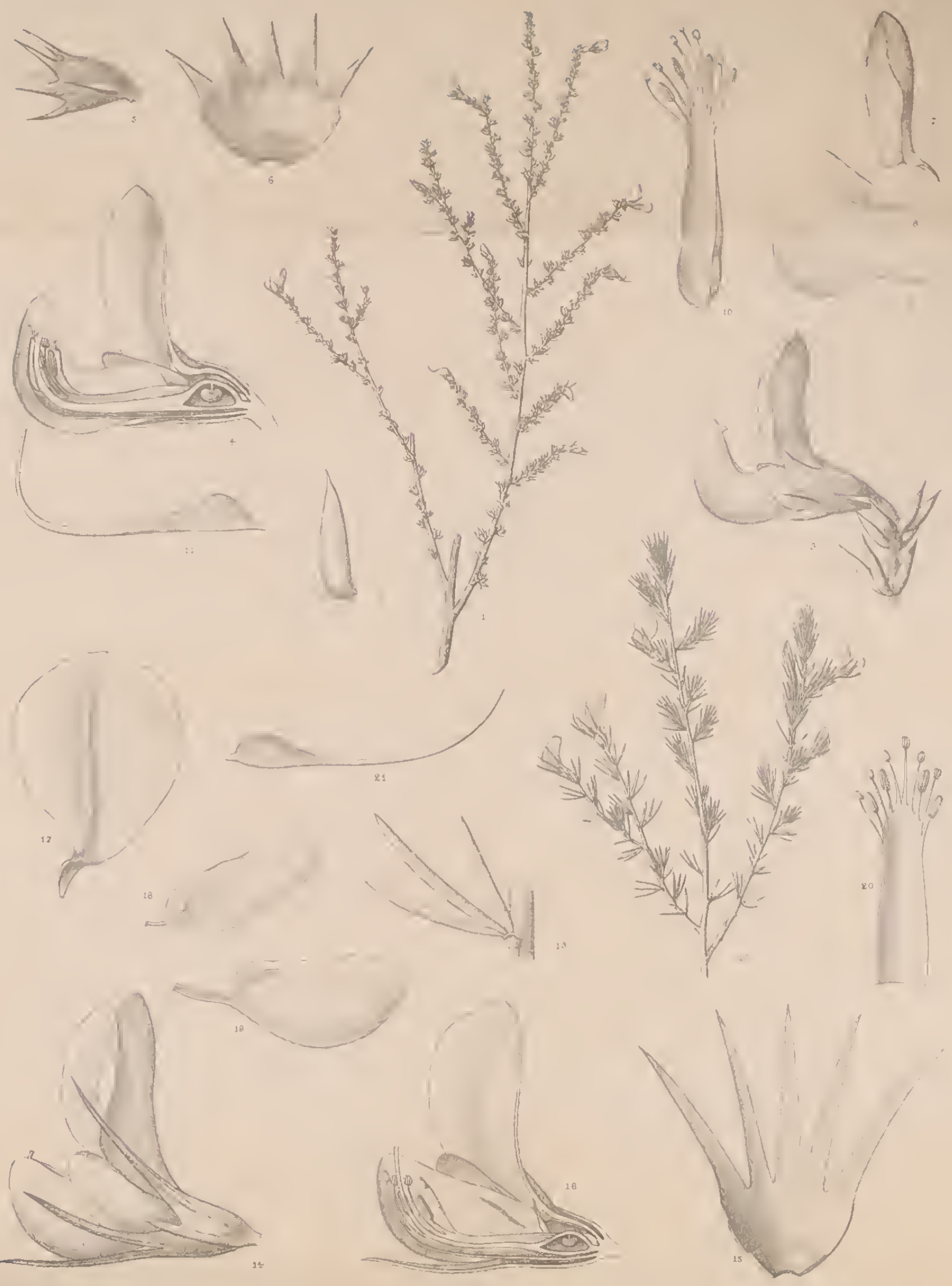

A d'A.prevel, ed nat del. 


\section{Aspalathus Ieptoptera Bolus}

ex Mac Owan in Journ. Linn. Soc. XXV (1889) p. 160.

Petite plante frutescente, rigide, rameuse, couchée, à rame:ıux densément feuillus, pubescents. Feuilles cylindriques, à extrémité aiguë-spinescente, glables ou munies à la base d'un coussinet velu, de $2 \mathrm{~mm}$. environ de long. Fleurs terminales, solitilires, subsessiles de 7 à $8111 \mathrm{~m}$. de long. Calice velu, muni à la base de bractées lancéolées environ aussi longues que le tube du calice, celui-ci velu, extérieurement, glabre intérieurement, dents subégrales, aiguës, velues sur les deux faces, un peu plus courtes que le tube. Corolle deux fois aussi longue environ que le calice; étendard velu-sericé sur le dos, cilié, courtement ongruiculé; ailes oblongues, obtuses, beitucoup plus courtes que la carène, celle-ci recourbée presque à angle droit, velue sur le dos, obtuse au sommet. Ovaire ovoïde, renflé supé-

Explication des figures 1 à 11 de la planche XLIII.

Aspalathus leptoptera Bolus.

Fio. 1. - Rameau fleuri (1/1).

F1G. 2. - Feuille isolée (3/1).

Fıg. 3. - Extrémité d'un rameau avec fleur épanouie (7/1).

FIa. 4. - Fleur épanouie, fendue (10/1).

Fig. 5. - Calice vu de profil, avec bractéoles (10/1).

Fro. 6. - Calice fendu et étalé vu par la face interne (2/1).

FIa. 7. - Étendard (7/1).

F16. 8. - Aile (7/1). 
lieurement, monosperme, velu, terminé en un style allongé, glabre, à stigmate peu développé.

Hab. - Région austro-occidentale [Dans les environs de Ceres (leg. H. Bolus, 1888)].

Observations. - L'A. leptoplera, signalé pour la première fois en 1889, est, comme le déclare M. le prof. H. Bolus, voisin de l'A. divaricata 'Thunb., mais il s'en différencierait facilement par' ses fleur's généralement solitaires et par les ailes de la corolle relativement très courtes. Il appartient done à la même subdivision du genre que l' $A$. cliffortioides Bolus dont nous avons essayé d'établir les affinités plus lıaut : toutefois la structure des feuilles cylindriques-épineuses, spinescentes, courtes, range tout naturellement cette espèce dans le voisinage des $A$. pungens Thunb., acuminata Lam. et divaricala Thunb.

Ceste dernière espèce est très répandue dans la colonie et r'elativement très variable; on lui reconnait trois variétés sous les caractères desquelles nous n'avons pas à insister ici.

Les caractères différentiels que nous avons rappelés plus haut, nous sont fournis par les notes de M. H. Bolus, qui a pu comparer sur le vif les échantillons de l'A. leptoptera avec de nombreux spécimens d'A. divaricala.

FIg. 9. - Carène $(9 / 1)$.

Fig. 10. - Androcée (10,1).

Fin. 11. - Oraire, style et stigmate (11/1). 


\section{Aspalathus Schlechteri Bolus}

ex Schlechter in Engler Bot. Jahrb. XXVII (1899) p. 146.

Petite plante frutescente à rameaux couchés ou dressés, en partie nus, anx cicatrices laissées par les feuilles tombées ou se trouvent de petites touffes de poils spinulifères. Feuilles fasciculées, aiquës-épineuses, étalées, linéaires sétacées, ciliées, à poils assez allongés, de 5 à 7 nmm. de long Fleurs latérales ou parfois terminales, de 7 à 11 lnm. de long, à calice relu muni à la base de bractéoles, sétacées, ciliées, environ aussi longues que le tube calicinal, lobes linéaires, uninervés, aigus, 2 à 2 l/2 fois aussi longs que le tube, velus sur les deux faces, tube du calice glabre sur la face interne. Étendard obovale, obtus, séricé extérieurement, cilié, glabre intérieurement; ailes oblongues, glabres, beancoup plus courtes que l'étendard; carène subgéniculée, réricée sur la suture inférieure, un pen plus courte sue l'étendard. Ovaire ovoüle-elliptique, velu, uni

Explication des figures 12 à 21 de la planche XLIII.

Aspalathus Schlechteri Bolus.

F10. 12 - Extrémité d'un rameau fleuri (1/1).

Fro. 13 - Fascicule de feuille (1/1).

Fig. 14. - Fleur épranouie (5/1).

FIG. 15. - Calice fendu et étalé (5/1).

Fia. 16. - Corolle en coupe longitudinale (5/1).

F1a. 17. - Étendard (5/1).

Fra. 18. - Aile (5/1). 
ou hiovulé, terminé par un style grêle, glabre ; légume obliquement ovoïle, aigu, velu hispide.

\section{Hab. - Dans la région austro-occidentale [Près de Caledon, dans le Zwarteberg (R. Schlechter)]}

Observations. - Cette espèce appartient d'après son auteur au groupe des "Laterales" bien que, comme le montre notre figure. les feuilles puissent se présenter parfois en position quasi-terminale: elle se fait remarquer par la sorte d'épine recourbée subpersistante à l'aisselle de laquelle se développe le fascicule des feuilles.

Les caractères accordés par Harvey à ce groupement sont :

Feuilles fasciculées, cylindriques ou trigones. Fleurs subsessiles, latérales. Ovaire 2-4 ovulé. Fruit villeux, exsert, obliquement ovale ou lancéolé, généralement turgide, étalé ou réfléchi à maturité.

Si nous cherchons les affinités de cette plante, nous royons que jar scs touffes de feuilles non munies d'une épine centrale, ses feuilles courtes de moins de $8 \mathrm{~mm}$. de long. elle parait devoir se ranger dans le voisinage des $A$. lancifolia Berg., canescens L., et frankenioides DC., dont les deux dernières sont particulièrement variables et semblent aroir surtout des analogics avec la plante que nous figurons; mais l'A. canescens a les feuilles encor'e plus longues, 6 à $8 \mathrm{~mm}$. et l'A. fircinkenioides les aurait un peu plus courtes, 2 à 4 mm. De plus, aucune le ces deux espèces, à en juger du moins d'après les descriptions, ne posséderait la sorte d'épine recourbée, à l'a:sselle de laquelle se développe le fasciculc foliaire et sur laquelle l'auteur insiste.

Fig. 19. - Carène (5/1).

Fig. 20. - Androcée (5/1).

Fia. 21. - Ovaire et style (8/1). 


\section{.

.



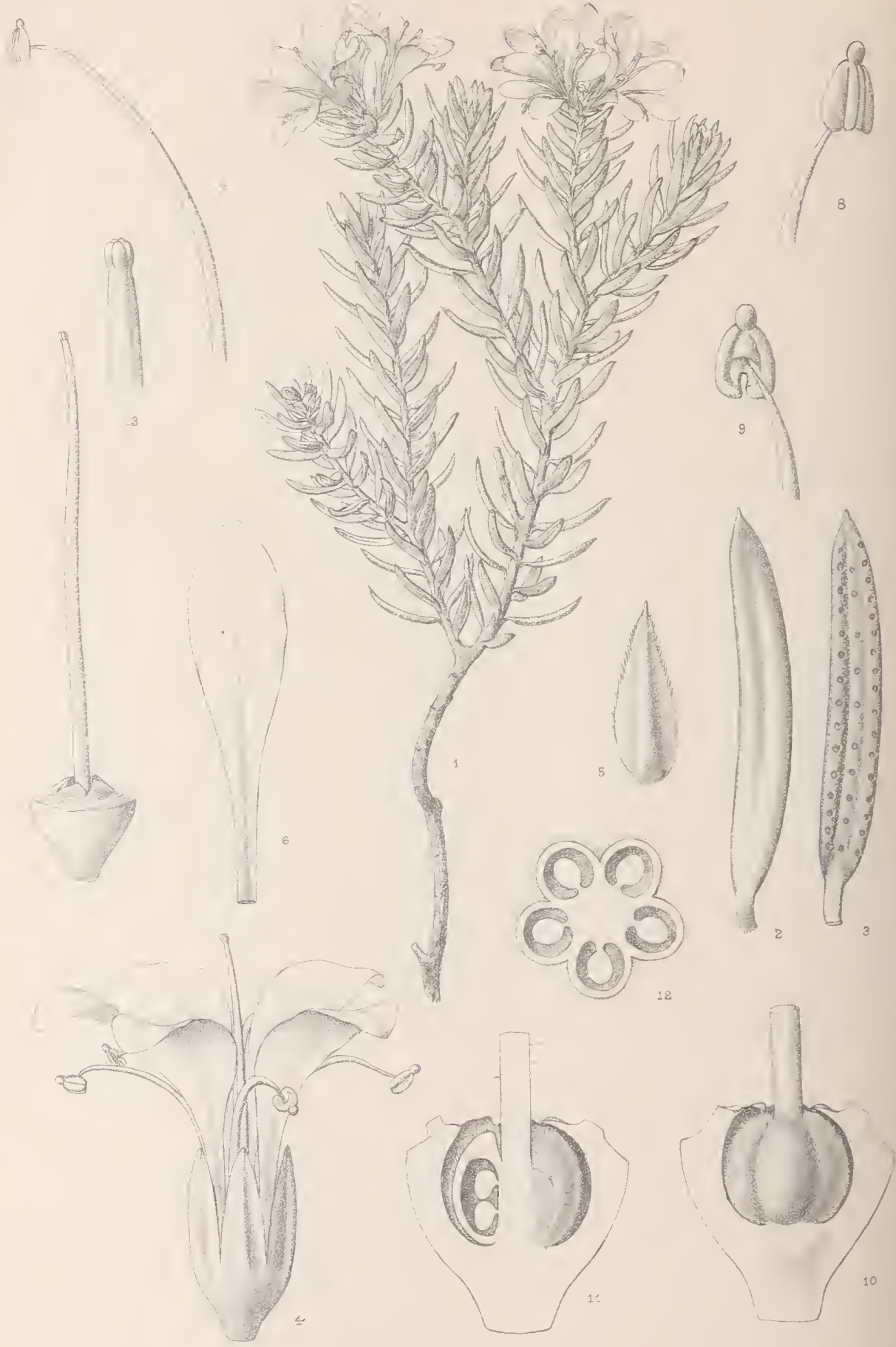

A.d'Apreval,ad nat del

Etabl ${ }^{\text {ts }}$ Minot. Paris.

Phyllos MA CAPENSIS Bolus 


\section{Phyllosma capensis Bolus}

in Engler Bot. Jahrb. XXIV (1897) p. 547.

Plante suffrutescente, à rameanx cylindriques, dressés, mivés de feuilles vers la hase, velus et feuillus au sonımet. Feuilles étalées-dressées, plus ou moins recourbées, imbriquées, épaisses, munies d'un cal au sommet, puberuleuses à l'état jeune, devenant grlalures, luisantes, courtement pétiolées, d'environ $1 \mathrm{~cm}$. de long, munies de chaque côté de la nervure médiane d'une ligne de glandes immercrées dans le tissu, Fleurs terminales, solitriires ou fasciculées, comrtement pédicellées; calice à 5 parties, de 5 à $6 \mathrm{~mm}$. de long, à lobes ovales. subacuminés, carènés, ciliés sur les bords. Pétales étalés, de 1 à 1,5 cın. de loug, onguiculés, spatulés, à lame obtuse de $9 \mathrm{~mm}$. environ de diamètre. Disque entourant l'ovaire, à 5 dents; étamines insérées sur le bord externe du disque, environ aussi longues que les pétales; anthères elliptiques à filet se terminant au delà

\section{Explication des figures de la planche XLIV.}

Fig. 1. - Rameau fleuri (1/1).

Fig. 2. - Feuille vue de dos (6/1).

Fro. 3. - Feuille vue de face $(6 / 1)$.

Fra. 4. - Fleur épanouie (3/1).

Fic. 5. - Dent calycinale (4/1).

Fig. 6. - Pétale (3/1),

Fí. 7. - Base du calice, entourant l'ovaire surmonté du style (5/1).

Fia. 8 et 9 . - Anthères vues de face et de dos (1/10). 
des loges prar une glande globuleuse. Ovaire 5-lobé, villeux, terminé par un style filiforme, éparsement hispide, à stigmate 5-lobulé, terminal. Ovules au nombre de 2 dans chacune des loges.

Hab. - Région du Karroo [près du Koudeberg dans le Wupperthal, district Clanwilliam (H. Bolus et R. Schlechter)].

Observations. - Le Phyllosma capensis est, jusqu'à ce jour, l'unique représentant du genre qui a été décrit en même temps que l'espèce, par M. H. Bolus, dans les "Plantae Schlechterianae novæ vel minus cognitæ "(loc. cit.).

Le genre Phyllosma appartient à la famille des Rutacece et trouve sa place dans la section des Diosmea, auprès des genres Macrostylis Bartl. et Wendl et Diosma L. D'après son auterr', il peut ètre considéré comme intermédiaire entre ces deux genres dont il s'en différencie cependant assez nettement. En effet, dans la subdivision des RutoideaDiosmex-Diosmino, - auquel appartiennent les deux genres cités plus haut, et qui est caractérisé par les carpelles au nombre de 5 à 6 , les fleurs hermaphrodites, à endocarpe se séparant totalement, - on peut considérer deux groupes. L'un est constitné par les genres dont les fleurs possèdent 5 étamines fertiles et 5 staminodes et dans lequel nous rencontrons les genres Barosma, Agathosma, Adenandra, Colconema, Acmadenia sur lesquels nous avons déjà attiré l'attention dans les "Icones selectie "; l'autre groupe, qui nous intéresse davantage, est caractérisé par l'abscnce de staminodes et par les fleurs toujours terminales : il ne renferme que les trois genres Diosma, Macrostylis et Euchaetis; chez le premier genre les petales sont sessiles, chez les deux autres ils sont onguiculés, les Macrostylis ayant le style allongé et les Euchaetis le style court.

C'est évidemment dans ce second groupe que vient s"intercaller le Phyllosma capensis et la clef analytique proposée par M. le prof. Engler dans les Pflanzenfamilien pourrait donc être modifiée comme suit :

Fig. 10. - Base de la fleur, dont une partie du calice est enlevée pour laisser voir l'ovaire en entier (11/1).

Fıs. 11. - Coupe longitudinale du calice et de l'ovaire (11/1).

Fig. 12. - Coupe transversale de l'ovaire (8/1).

F1G. 13. - Extrémitè du style avec stigmates (12/1). 
B) Fleur's privées de staminodes; fleur's toutes terminales.

(є) lileur's à pétales sessiles . . . . Diosma.

b) Fleur's à pétales onguiculés.

a) Style allongé, à stigmate réduit, entiel.

1) Pétales velus sur la face interne . . . . . . . Macrostylis

2) Pétales glabres . . . . Phyllosma.

ß) Style court, à stigmate capité . . Euchaetis.

La fleur du Phyllosma capensis, est plus développée que celle des Macrostylis; la longueur dos pétales et leur glabréité permettent à simple examen d'écarter la plante nouvelle des représentants de ce genre.

De ce qui précède, il résulte que M. H. Bolus a parfaitement appréciée l'importance des caractères floraux du Phyllosma capensis, en créant pour cette plante un nouveau genre et que nous reconnaissons toute la valeur de sa diagnose. Le savant botaniste du Cap, en nous communiquant quelques notes complémentaire, insiste également sur le port et l'apparence générale de la plante, circonstances qui dans le cas présent, ont eu pour lui une importance très particulière, mais que nous ne pouvons malheureusement pas rendre sur la planche XLIV dessinée uniquement d'apı'ès des matériaux d'herbier. 



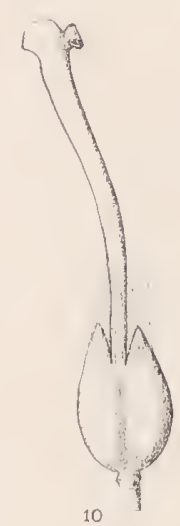

Sin
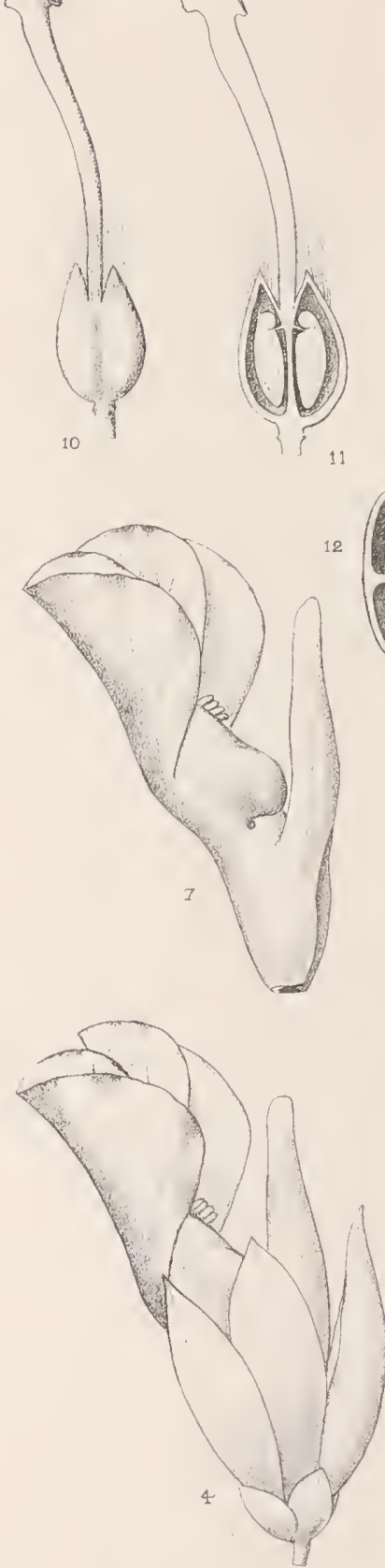

A.d'Appeval,ad nat.del.
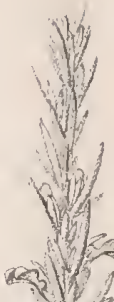

1, 促
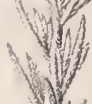

19

$1 \mathrm{~N}$

W
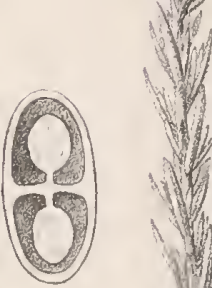

11

14

I

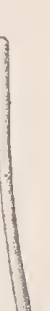

Wy

Win

in

w

WI
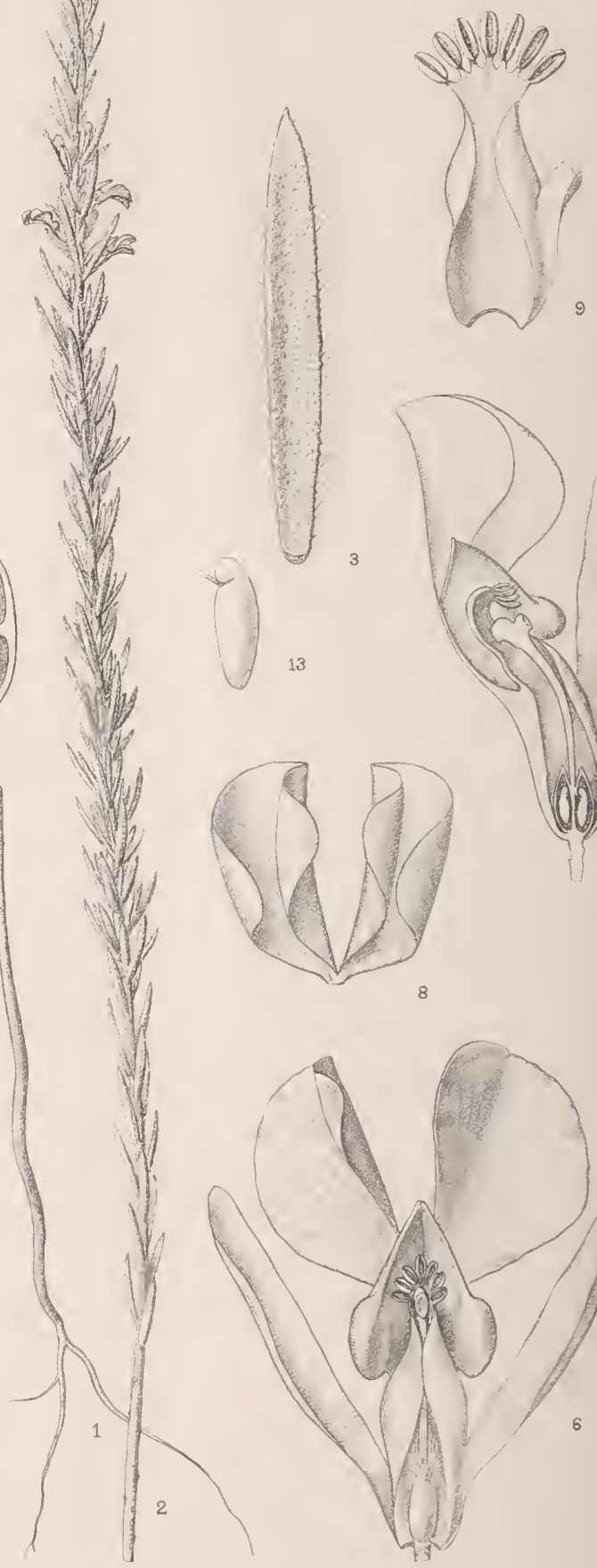

13
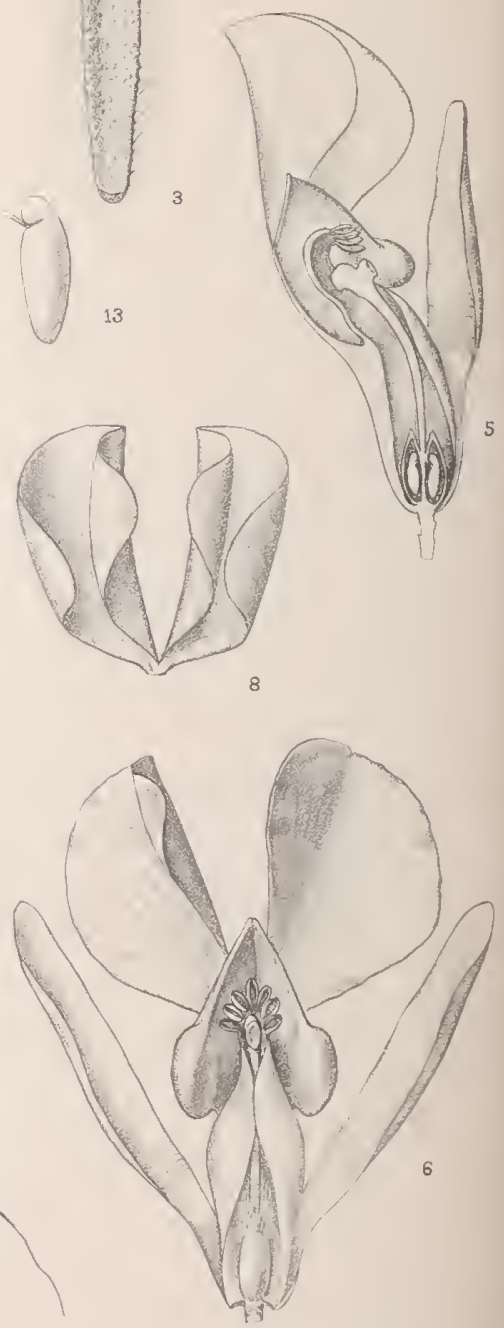

Etabl' Minot. Paris

$$
\text { MURALTIA SPICATA Bolus }
$$




\section{Muraltia spicata Bolus}

ex Transact. of the South African Philos. Soc. vol XVI, part. 2 (1905) p. 135.

Petite plante sous-frutescente, dressée, atteignant $45 \mathrm{~cm}$. do haut, peu ramifiée, ì l'umeaux dressés, cylindriøues. Feuilles de 7 i l $12 \mathrm{~mm}$. le long et de 1 à $1,8 \mathrm{~mm}$. de liuge, sessiles, génćralement dresscees, presque imbriquées, étroitement oblongues ou oblancéolres, subobtuses, canaliculées supérieurement, carénées sur le dos, plus ou moins tuberculeuses-rugueuses, à poils subétalés, blanchâtres. Fleurs disposées en un épi terminal, interrompu, de 3 à $10 \mathrm{~cm}$. de long et de $1 \mathrm{~cm}$. environ de large. Sépales oblongs lancéolés, aigrus, concaves, glabres, subcarénés, de 4 à 5 min. de longg. Pétales latéraux dressés, ongoniculés, soudés jusque vers le milieu, ligulés dans la partie supérieure, a ligule obtuse; carc̀ne de $8 \mathrm{~mm}$. de long, onguiculée à la hase, terminée en lobes subréniformes de $5 \mathrm{~mm}$. de long et $3 \mathrm{~mm}$. environ de large dépassant les pétales.

Explication des figures de la planche XLV.

Fig 1 et 2 . - Plante fleurie (1/1).

Fra. 3. - Feuille vue par sa face inférieure (6/1).

Fı́. 4. - Fleur épanouie (8/1).

FIG. 5. - Coupe longitudinale de la fleur, calice enlevé $(8 / 1)$.

Fıa. 6. - Corolle dont les lobes externes ont été enlevés $(8 / 1)$.

Fits. 7. -- Corolle, vue de profil (8/1).

Fro. 8 - Lobes anlérieurs de la corolle $(8 / 1)$.

Fio. 9. - Androcée (15/1). 
Ovaire biloculaire, à loges uniovulées, terminées au sommet par des toulfes de poils, allongé, glabre, s'élarorissant au sommet. Capsule ohovale, velue au sommet, à cornes presque aussi longues que la capsule qui mesure $5 \mathrm{~mm}$. de long; graines glahres on très éparsement pubescentes.

Hab. - Région autro-occidentale [(Environs de Elim, district de Bredasdorp. (H. Bolus); régions montagneuses des environs de Napier (Guthrie) et dans les collines des environs de la Koude Rivier (Scblechter)].

Observations. - D'après la courte notice qui accompagne la description publiée par M. H. Bolus et dont nous avons donné un résumé ci-dessus, le $M$. spicata serait voisin du $M$. thymifolia Thunb., dont il se différencierait par le port plus grêle, les rameaux plus cylindriques et les fleurs plus grandes.

Cette dernière espèce était caractérisée dans la Flora capensis, I p. 97, par ses mots "Robust divaricrile; leaves ovato-lanceolate or ovate oblong".

On pourrait donc, d'après la description de M. Bolus, mettre pour le $M$. spicata, les caractères suivants en opposition :

"Slender virgate, leaves angusto-oblong or oblanceolate."

Mais, com:re on le voit, ce sont des nuances difficiles à saisir, et c'est la raison pour laquelle nous avons été amenés à faire figurer cette plante. Dans le genre Muraltia dont relativement bien peu d'espèces ont été figurées, il devient très nécessair' le préciser les caractères; car d'après toutes les données, ces espèces sont très variables et on peut se demander si une étude attentive, sur de nombreux matériaux de provenance différente, n'amènerait pas une réduction plus ou moins notable des types spécifiques.

Fia. 10. - Oraire, style et stigmate (15/1).

Fra. 11. - Corne longiturlinale de l'ovaire (16/1).

F1G. 12. - Coupe transversale de lovaire (25/1).

Fı́. 13. - Ovule isolé (25/1). 


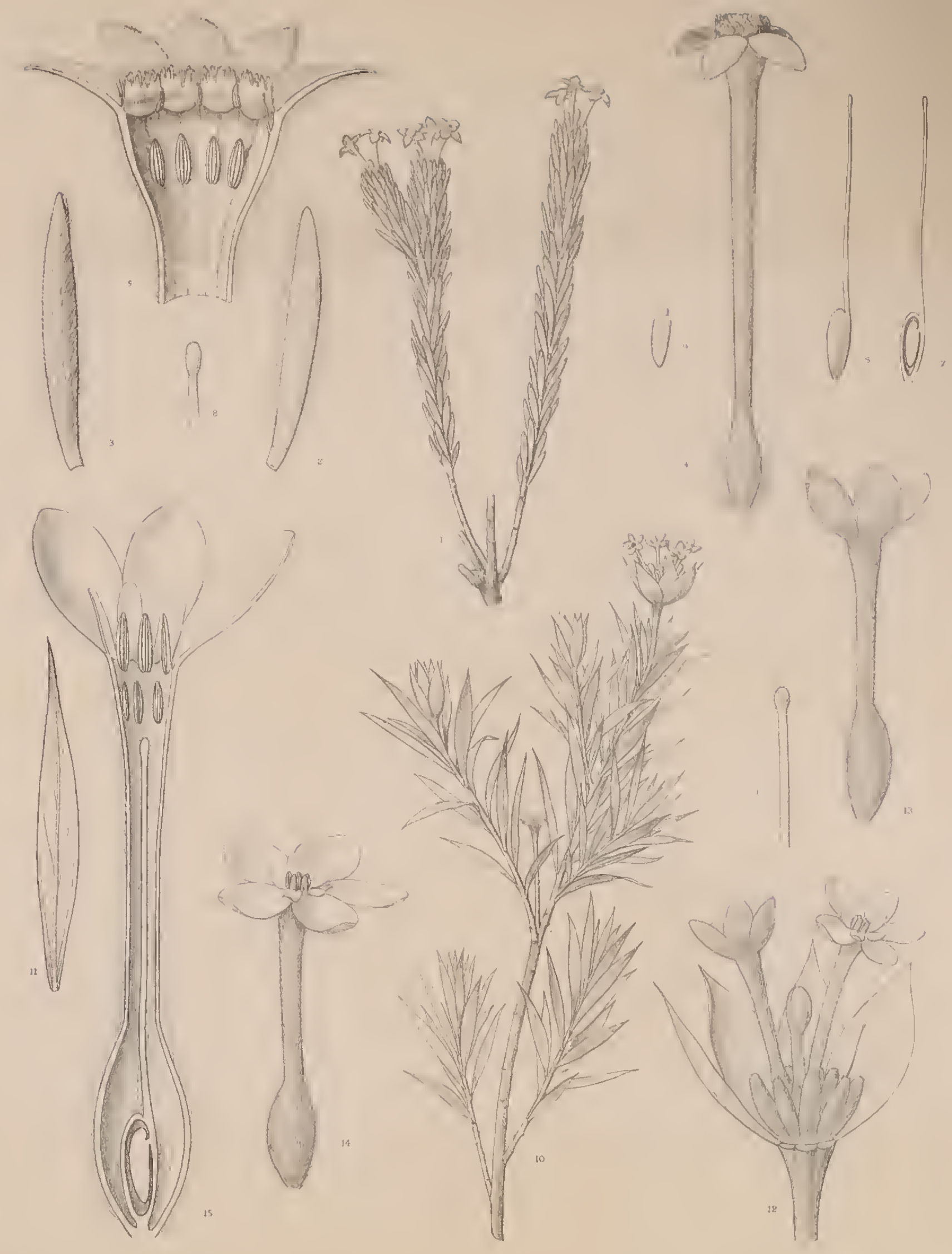




\section{Gnidia pulvinata Bolus}

in Trans. of the South African Philosophical Society XVI, 2 (1905) p. 142 .

Plante sous-frutescente de 3 à 4 pieds de liaut, à lameaux rigides, pubescents à l'état jeune devenant rapidement glabres et grisâtres, garuis des cicatrices laissées par la chute des feuilles. Feuilles rapprochées vers le sommet des rameaux, opposées ou éparses, imlriquées plus ou moins fortement surtout à l'état jeune, étroitement lancéolées, longuement atténuées, subolutuses, coriaces, plus ou moins rugueuses, très densément velues à l'état jeune, devenant glabres avec l'âge, de 1 à $1,5 \mathrm{~cm}$. de long et $2 \mathrm{~mm}$. de large. Fleurs disposées par 3 à 5 en glomérules terminaux, non involucrés, sessiles ; calice à tube grêle, r'enflé à la base et à la gorge, densément velu sur la fice externe.

\section{Explication des figures 1-9 de la planche XLVI.}

Gnidia pulvinata Bolus.

FIG. 1. - Fragment de plante fleurie (1/1).

FIG 2. - Feuille vue de face $(7 / 1)$.

Fı. 3. Feuille vue de dos $(7 / 1)$.

Fig 4. - Fleur épanouie (7/1).

Fig. 5. - Sommet du périanthe feıdu et étalé (1 $1 / 1)$.

Fig. 6. - Ovaire (i/1).

Frg. 7. - Coupe longitudinale de l'ovaire (7/1).

F1G. 8. - Extrémité du style avec stigmate (20/1).

Fia. 9. - Ovule $(8 / 1)$. 
de 1 à $1,2 \mathrm{~cm}$. de long et d'un peu plus de $1 \mathrm{~mm}$. de diamètre, segments plus ou moins réfléchis, ovales, lancéolés, aigus, densément velus sur les deux fices, d'un pourpre foncé de $6 \mathrm{~mm}$. de longr et de $25 \mathrm{~mm}$. de large. Pétales alternatisépales, formant 4 petites écailles, éprissies, cliarnues, denticulées, glabres, insérées à la gorge, formant un anneau complet dont les denticulations filiformes diminuent de longueur vers l'extérieur. Étanines disposées sur deux rangs, anthères du rang inférieur parfiaites, celles du rang supérieur arortées alternant arec celle du r'ang inférieur et oppositi-sépales.

Hab. - Région austro-occidentale : District de Bredasdorp sur les montagnes entre Caledon et Elim à environ 600 pieds d'altitude (Bolus, n. 9,238) et près de Koude Rivier, à 1,000 pieds (R. Schlechter, n. 9,619).

\section{Gnidia transvaaliensis Gilg nor. sp.}

in Herbario Hort. Bot. Conimbricensis, coll. F. Quintas.

Gnidia transvaaliensis; fruticulus, rami diffusi, rigidi, glabri, cicatricoso-tuberculati ; folia subspar'sa, suberecta, lanceolata, acuta, coriacea subpungentia, glabra, 15 à $22 \mathrm{~mm}$. longa et usque $4 \mathrm{~mm}$. lata; flores terminales, in glomerulis, circ. 10 floris, involucrati, pedicellati; bracteis ovato-lanceolatis, acuto-pungentis, circ. $12 \mathrm{~mm}$. longis; calicys tubus subgracilis fauce pauci ampliatus, extus dense tomentosus, circ. $9 \mathrm{~mm}$. longus, segmentis patentibus, ovatis vel obovatis, rotundatis supra glabres centibus infra dense pubescentibus, 2,5-3 mm. longis et circ. $2 \mathrm{~mm}$. latis; petalis squamatis; circ. $1 \mathrm{~mm}$. longis, integris, glabris, antheræ inferiores perfectæ minores, superioribus majores perfectæ, exsertæ.

Plante arborescente, à rameaux diffus, rigides, glabres, munis de cicatrices tuberculiformes, traces des feuilles tombées; celles-ci persistantes vers l'extrémité des rameaux, éparses, dressées ou dressées-étalées, lancéolées, aiguës, coriaces, presque piquantes, glabres, de 15 ì $22 \mathrm{~mm}$. de long et d'environ $4 \mathrm{~mm}$. de large. Fleur's réunies en glo- 
mérules involncrés, terminaux, d'au moins 10 fleurs, portés par un pédicelle plus on moins allongée fractées involucrales ovales-lancéolées aiguës, de $12 \mathrm{~mm}$. environ de lonğ, au nombre de 7 environ. Tuhe du calice assez crrèle, légèrement renflé à la base et vers la gorge, se désirticnlant à la base, un pen an-dessus du nivean du sommet de l'ovaire, densément tomenteux sur lis filce externe, de 9 mmi. environ de long, à segrments étalés, ovales ou obovales, arrondis an sommet, glabres sur la fince supérienre, densément velus sur lis face infériemre, de 2,5 it $3 \mathrm{~mm}$. de

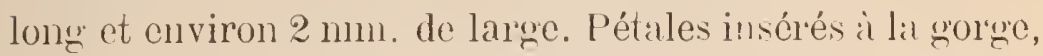
stuamiformes, environ 3 fois plus courts yue les sépales, entiers, glabres. Étamines sur deux rungs, le rang inférieur logé dans le tube ver's son sommet à anthères parrfaites plus petites que celles des étamines du lang supérieur, celles-ci également parfaites, insérées à la gorore et exsertes. Ovaire glabrescent i style latéral, plus court „que le tube, à stigmate légèrement renflé en massue.

Hab. - Région du Kalahari [Transvaal, Krokodil rivier, juin 1893 , (leg. F. Quintas, n. 229)].

Observations. - Les deux espèces que nous avons figurées ici appartienn nut au même sous-genre Eugnidia Endl.. caractérisé par ses capitules floraux terminaux, mais à deux sections différentes, créées dans ce sous-genre par M. le prof. E. Gilg, les Exinvolucratoe et les Involucrata.

I)'après les quelques renseignements que M. le $I^{r} \mathrm{H}$. Bolus a joints

\section{Explication des figures 10-16 de la planche XLVI.}

Gnidia transvaaliensis Gilg.

FiG. 10. - Rameau fleuri $(1 / 1)$.

Fig. 11. - Feuille 4/1).

F1G. 12. - Capitule floral à bractées extérieures en partie enlevées (3/1).

Fitg. 13. - Fleur épanouie (5/1).

F'ı. 14. - Fleur épanouie et lobes du périanthe ètalés (5/1).

F1a. 15. - Coupe longitudinale de la fleur (10/1).

Fí 16 - Extrécitité du style avec stigmate (20/1). 
à la description dont nous venons de donner une traduction, le $G$. pulvinatc est une espèce affine des $\dot{G}$. scabrida Meisn. et pubescens Berg., mais constitue néanmoins une plante très distincte par les caractères floraux.

C'est dans la première section que se classe le $G$. pulvinata et il répond bien à la diagnose de la section : capitule entouré de feuilles ordinaires sans involucre particulier. C'est d'ailleurs dans ce groupe que se rangent aussi les $G$. scabrida et pubescens.

Quant au $G$. transvaaliensis Gilg, c'est bien un représentant de la section Involucratce Gilg: Capitule entourée de bractées différant, des feiilles caulinaires. Les figures 10,11 et 12 de notre planche XLVI permettent de sc r'endre compte, à leur aspect différent, des feuilles normales et des bractées réceptaculaires.

C'est non seulement par la disposition des fleurs que se différencient les deux espèces que nous figurons ici, mais encore par la forme des pétales qui dans le $G$. pulvinata sont très courts, épais, frangés sur le bord, formant une couronne continue à la gorge du tube calicinal ; chez le $G$. transvaaliensis ces pétales sont étalés, pétaloïdes et non épaissis.

Nous n'avons pu trouver de description de cette dernière espèce que nous avons reçue dans un envoi de matériaux dherbier du Musée de Coïmbre. C'est une des raisons pour lesquelles nous avons tenu à en faire quelques analyses qui permettront à ceux qui voudront entreprendre des études monographiques de ce genre, encore très embrouillé, de se faire une idée des caractère's spéciaux dc cette plante. 


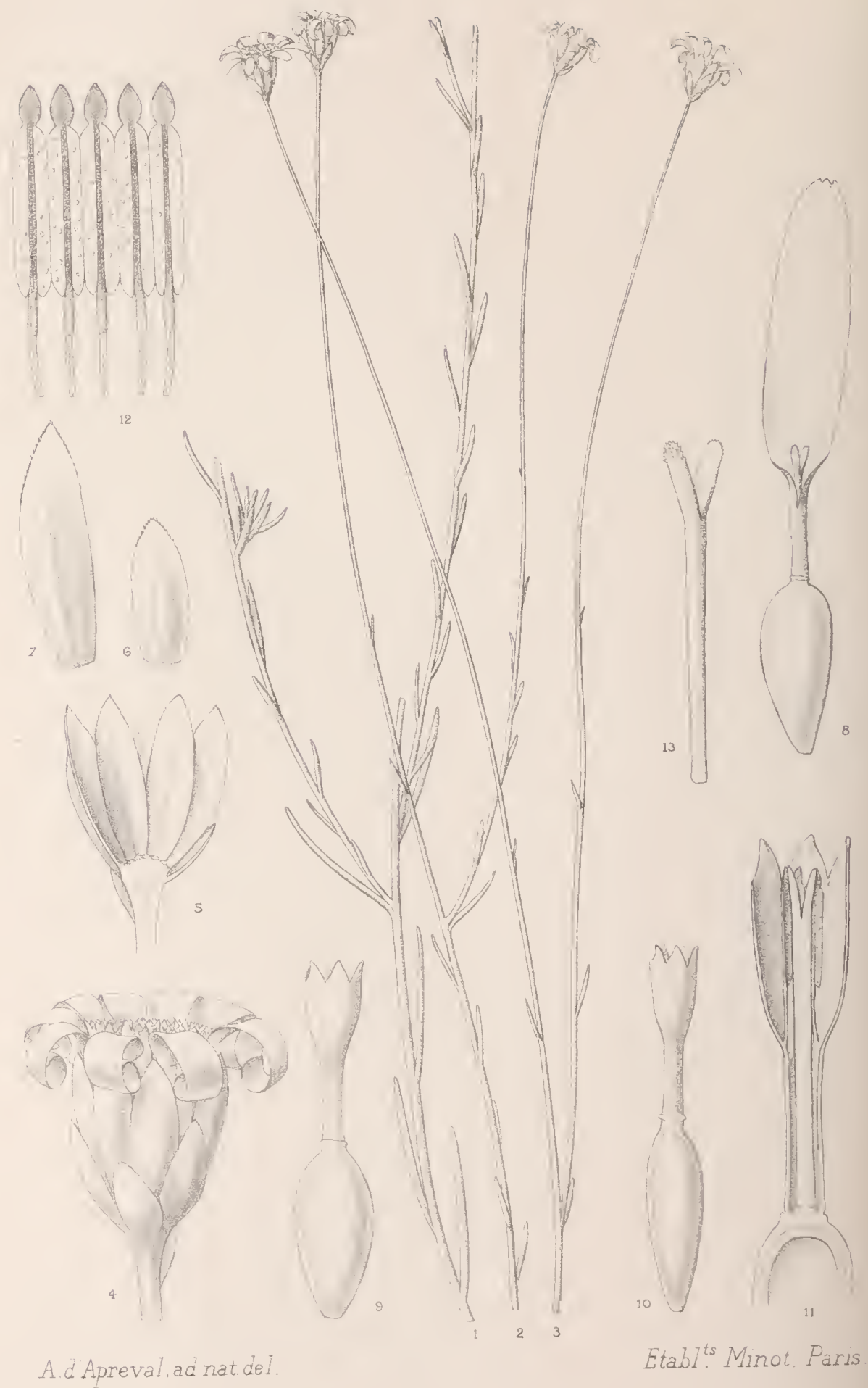




\section{Gymnostephium læve Bolus}

in Trans. of the South Afr. Philos. Society, XVI, 2 (1905) p. 138.

Petite plante grêle, dressée, presque glalıre dans toutes ses parties, atteignant parfois $45 \mathrm{~cm}$. de liant, a tiges simples ou peu ramifiées, garnies de feuilles plus ou moins espacées, alternes, linéaires. subaiguës, uninervées, parfois plus ou moins dilatées à la base et semi-amplexicaules, de 2 à $3,5 \mathrm{~cm}$. de long et de $1 \mathrm{~mm}$. environ de large. Intlorescence terminale, à pédoncule 11 ule 8 à $12 \mathrm{~cm}$. de long, à bractées foliacées, distantes; capitule turbiné ou cyathiforme de $8,5 \mathrm{~mm}$. de long et de $9 \mathrm{~mm}$. environ de large. Involucl'e, formé parfois de $t$ séries de squames, bractées de la série intérieure prarfois au nombre de 13, cunéiformes, oblongues, subobtuses, lisses, obscurément nerviées, légèrement ciliées ver's le haut, les externes plus jetites mạis de même folme ; réceptacle alvéolé. Fleurons ligulés externes parfois au nombre de 14, à ligule oblongue, tridentée au sommet, de couleur lilas, atteignant $7 \mathrm{~mm}$ de long, à akènes glabres, lisses, privés généralement de soies,

\section{Explication des figures de la planche XLVII.}

Fig. 1, 2 et 3. - Rameaux de la plante fleurie (1/1).

Fıa. 4. - Capitule floral (8/1).

Fig 5 - Capitule fendu, privé de ses fleurs (8/1).

FIG 6. - Bractée externe de linvolucre (10/1).

Fto T. - Bractée interne de l'involucre (10/1).

Fıı. 8. - Fleuron ligulé (10/1). 
I:arement munis au sommet de 2 à 3 soies raides. Fleurous 1 ubuleux du disque parfois au nombre de 50 , jaunes, à tube court légèrement dilaté au sommet au-dessus de la libération des étamines, celles-ci à anthères apiculées, à apicules élargis en palette, généralement stériles. Akènes ordinairement privés de soies ou munies de quelques soies barbelées.

Hab. - Région austro-occidentale [Gydonne (H. Bolus) et Zandfontein, Koude Bokkeveld (R. Schlechter)].

L'habitat de cette plante, comme celui des deux autres Composées qui seront figurées ci-aprés, est assez difficile à déterminer avec précision, puisqu'il se trouve á cheval sur le confin de deux régions, mais elle appartient évidemment, par son type de végétation, à la région austro-occidentale.

Observations. - Le genre Gymnostephium appartient dans cette famille au groupe des Asterince, de la tribu des Asterce, à capitules généralement hétérogames, à fleurons de la circonférence généralement fertiles, à fleurons internes généralement stériles, akènes assez souvent privés de soies ou à soies réduites.

Comme le montrent les figures de notre planche, l'aig1'ette est nulle, ce qui se présente surtout dans trois genres : Psilactis A. Gı ay, du Mexique; Charicis Cass., de l'Afrique australe et Gymnostephium; mais dans ce dernier genre seul les fleurs centrales du capitule sont stériles, tandis que dans les deux autres genres il $y$ en a toujours un certain nombre qui sont fertiles.

Les six espèces qui constituent le genre se classent, d'après Harvey (Flora capensis III p. 67), en deux groupes dont les caractères se basent sur la glabréité ou la pubescence des écailles de l'involucre, et sur la présence d'une carène.

Ce sera dans le premier groupe à écailles glabres que nous devons classer la plante qui nous occupe; les caractères "Involucral scales glabrous or nearly so, flat, marked with 3 glandular ribstria "ne correspondent peut-être pas tout à fait, et en effet les stries glandulaires ne sont guère marquées; cependant ces caractères sont mieux appropriés

Fig. 9 et 10. - Fleurons tubuleus (10/1).

Fig. 11. - Coupe longitudınale d'un fleuron tubuleux (29/1).

FIG. 12. - Androcée fendu et ètalé (35/1).

Fı。. 13. - Style et sligmates divergents 20/1). 
à la plante que nous figurons que eeux de la subdivision opposée dont les éeailles sont hispides ou pubescentes.

Des trois espèees qui eonstituent la première subdivision $G$.gracile Less., fruticosum I) C. et angustifolium IIarv., les deux premirires sont caractérisées par des feuilles à bords révolutés, la lroisième par des feuilles canaliculées et earénées : e'rst done de cette dernière qu'il faudra rapprocher le G. lave. Les deux espèces semblent, d'après les deseriptions, de la Flora capensis, avoir une grande analogie; mais nous n'avons pu comparer les éehantillons recueillis par Zeyher dans les environs de la rivière Zonder Einde, sur lesquels ces descriptions ont ćté faites, avec les spéeimens authentiques du Gymnostephium lave de M. H. Bolus. 



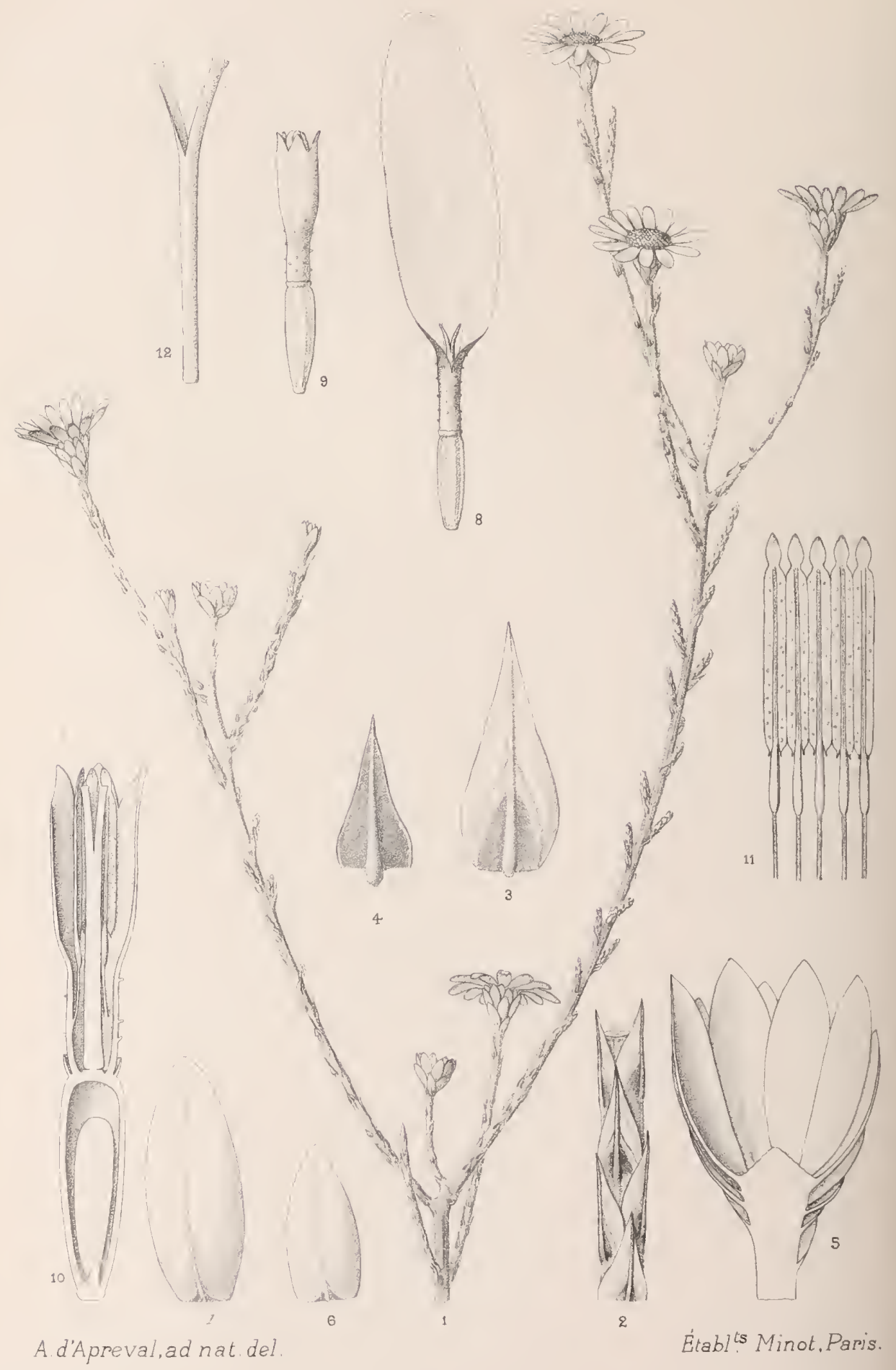

PHYMASPERMUM APPRESSUM Bolus 


\section{Phymaspermum appressum Bolus}

in Trans. of the South Afr. Philos. Soc. XVI, 2 (1905) p. 138.

Plante sulliutescente, dressée, rameuse, atteignant plus le 2 mètres dle liaut, à rameaux ascendants, assez grêles, feuillus, à nervures foliaires décurrentes le long des tiges; rameaux inférieurs généralement stériles, courts, de 7 à $15 \mathrm{~mm}$. de long, à feuilles densément imbriquées, rameaux suprérieur's florifères plus allongés atteignant $10 \mathrm{~cm}$. de long, à feuilles plus espacées à mesure que l'on se rapproche du capitule. Feuilles sessiles, apprimées, ovales, assez épaisses, glabres, aiguës à l'état jeune, levenant longuement cuspiclées-acuminées avec l'âge, de 2 à $4 \mathrm{~mm}$. de long, cuspis membraneux; sétiforme, brunâtre, aussi long ou plus long que le limbe, à trois nervures visibles surtout sur la face inférieure, la médiane proéminente, épaissie, longuement décurrente, les latérales moins décurrentes et parfois même peu marıuées. Capitules terminant des rameaux courts ou allongés, terminaux, solitaires, formant par leur réunion des corymbes irréguliers.

\section{Explication des figures de la planche XLVIII.}

Fı́. 1. - Extrémité de la plante fleurie (1/1).

Fig. 2. - Fragment de tige avec feuilles imbriquées (4/1).

Fia. 3 et 4 . - Feuilles des rameaux et des ramuscules (8/1).

F1G. 5. - Capitule privé de ses fleurs, vu en coupe $(5 / 1)$.

Fra. 6. - Bractée extérieure du capitule (5/1).

F1G. 7. - Bractèe intérieure du capitule (5/1). 
Involucre lrémispliérique, à squames dressées, imbriquées, disposées environ sur 4 séries, glabres, papyracées, jaunâtres, les extérieures plus courtes que les intérieures, toutes à peu près de même forme, ovales, subobtuses, carénées; receptacle convexe, muni de quelques poils courts et articulés. Fleurons ligulés disposés au nombre de 10 à 14 en une série, ligules oblongues, blanches, de $7 \mathrm{~mm}$. environ de long, tridenticulées au sommet, akène réduit, non surmonté d'aigrette; fleurons tubuleux, jaunes, de $3 \mathrm{~mm}$. de long, au nombre de 40 à 50 , à tube court peu renflé, légèrement glanduleux, à 5 lobes subaigus; étamines à anthères apiculées, à apicule élargi. munies à la base d'une pointe recourbée; style à lobes divergents ì stigmates tronqués courtement ciliolés. Akènes subcylindriques, glanduleux, à 10 côtes, de $2 \mathrm{~mm}$. environ de long, à soies nulles ou remplacées par de petites dents, prolongements des côtes.

Hab. - Région du Karroo (sur le versant rocheux de la Zwartberg Pass, district Prince-Albert (H. Bolus).

D’après les communications que M. Bolus a bien voulu nous faire, l'espèce se rencontrerait sur les versants très rudes et peu explorés de la chaine de montagnes du Groote Zwartenberg sur les limites de la région sud-ouest et de celle du Karroo, mais a une altitude supérieure à celle de cette dernière région qui ne dépasse pas 3,500 pieds. Le savant botaniste estime toutefois quil y a lieu de l’assigner á celle-ci.

Observations. - Le genre Phymaspermum constitue avec les genres Lidbeckia Berg., Thamniophyllum Harv., Matricaria L., Lepidosthephium Oliv.. Chrysanthemum L., Phalacrocarpum Willk., Waldheimia Kar et Kir., un groupement dans lequel les capitules floraux donnent les caractères : les fleurons ligulés dépassent les bractées.

Parmi ces genres, le dernier possède des akènes couronnés par des

Fig. 8. - Fleuron ligulé (8/1).

Fia. 9. - Fleuron tubuleux (8/1).

FIG. 10. - Coupe longitudinale du fleuron tubuleux (16/1).

Fig. 11. - Androcée fendu et étalé (20/1).

Fig. 12. - Style et stigmates divergents (20/1). 
soies nombreuses, plus ou moins soudces à la base, ou par des écailles se terminant en soies, tandis que dans les autres genres les soies sont nulles ou l'akène est couronné de petites écailles parfois réunies en couronno.

Dans ce dernier groupement nous écartons le genre Phalacrocarpum par ses feuilles opposées et les espèces du genre Matricaria sont dos plantes herbacées. Chez les Lepidostephium et Chrysanthemum les bractées de l'inflorescence sont disposées sur un grand nombre de rangées; chez les autres genres, le nombre de rangées est réduit. Nous restons en présence de trois genres : mais, tandis que chez les Phymaspermum les fleurons ligulés sont fructifères, - chez les deux autres genres Lidbeckia et Thamniophyllum ces fleurons sont asexués et stériles.

Le genre Phymaspermum ne renferme que peu d'espèces, encore assez mal connues. D'après $\mathbf{M}$. Bolus, la plante nouvelle aurait certaine ressemblance avec le $P h$. junceum Less. (= Osteospermum junceum 'Thunb.), dont elle se distinguerait par ses feuilles petites, apprimées, ovales, épaisses; mais cette dernière espèce ne semble plus avoil' été recueillio depuis qu'elle a été trouvée par 'Thunberg et l'unique échantillon cunser'vé dans son herbier est, au dire de Harvey lui-même, très précaire.

M. II. Bolus considère également comme possédant certaines analogies avec son $P h$. appressum le $P h$. aciculare $(=$ Iocaste acicularis E. Nay.) assez rare dans les herbiers et dont nous n'avons pu confronter des échantillons avec le type de $\mathrm{M} \mathrm{H}$. Bolus qui a servi à l'établissement des figures analytiques de notre planclie. 



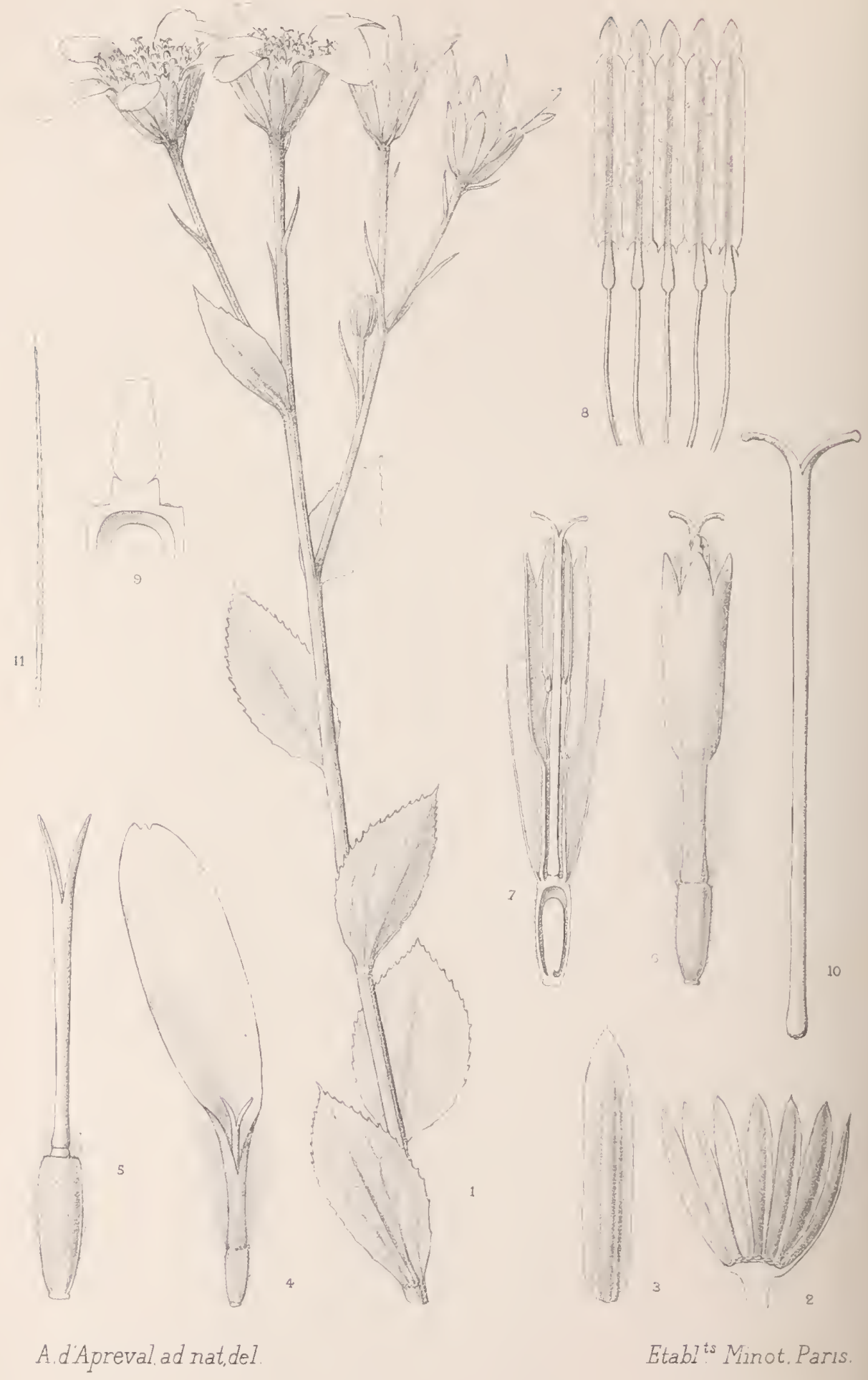

SENECIO SOCIORUM Bolus 


\section{Senecio sociorum Bolus}

in Journ. Linn. Soc. Bot. vol. XXIV (1887) Austro Africana Flora exsicc. n. 5434 .

Tiges herbacées, dressées ou subdécombantes, feuillues et naissant d'un rhizome renflé, striées, aranéeuses-tomenteuse à l'état jeune, devenant glabres; feuilles caulinaires sessiles, ovales-oblongues, de 2,5 à $4,5 \mathrm{~cm}$. de long et de 1 à $2,5 \mathrm{~cm}$. de large, subcunéiformes à la hase, aiguës au sommet, denticulées-serrées sur les bords, à nervures au nombre de 3 à 5 dès la base, à nervures secondaires nettement réticulées, glabres sur leș deux faces. Capitules floraux longuement pédicellés, formant, au nombre de 4 à 12, corymbe au sommet des tiges; pédicelle de 3 à $10 \mathrm{~cm}$. de long, bractéolé, à bractées lancéoléeslinéaires; calicule formé de squames linéaires de moitié environ aussi longues que l'involucre. Involucre subcylindracé, formé de 12 à 14 bractées linéaires, obscurément trinerviées, subtriangulaires au sommet, courtement ciliées, de 11 à $12 \mathrm{~mm}$. de long et $2 \mathrm{~mm}$. de large; réceptacle

\section{Explication des figures de la planche XLIX.}

Fig. 1. - Extrémité d'un rameau fleuri (1/1).

Fı. 2. - Capitule privé de ses fleurs $(2,5 / 1)$.

Fı. 3. - Bractée du capitule, vue par la face externe (4/1).

Fro. 4. - Fleuron ligulé (3/1).

Fıa. 5. - Ovaire et style du fleuron ligulé $(8 / 1)$.

Fio 6. - Fleuron tubuleux (4/1).

Fig. 7. - Coupe longitudinale du fleuron tubuleux $(4 / 1)$. 
alvéolé. Fleurons de la périphérie au nombre de 3 à 5 , ligules de 1 à $2 \mathrm{~cm}$. de long, oblongues, obscurément tridentées au sommet, akène velu surmonté d'une aigrette de poils jaunâtres, environ aussi longs que l'involucre; fleurs du centre au nombre de 28 environ. tuhullcuces peu exsertes, corolles à 5 dents; anthères appendiculées au sommet, à style à lobes divergents, courts et olıtus, akène striés sétuleux, à soies jaunâtres, denticulées.

Hab. - Région austro-occidentale [Mittchells Pass, près Ceres. á 1.300 pieds, avril 1885 (leg. H. Bolus et Mac Owan, n. 5,434 et Herb. nouv. Austr. Afr. n. 386)].

Observations. - Comme le fait très justement remarquer M. le $\mathrm{D}^{\mathrm{r}}$ Hoffmann, dans la revision des Pflanzenfamilien, on n'a pas encore réussi à donner une classification rigourouse des espèces du genre Senecio - et l'on en compte plus de douze cents - en sous-genres ou en sections; car les caractères qui ont de la valeur dans une région donnée, et qui permettent une classification des espèces propres à cette région, ne peuvent être employés avec succès quand on essaye d'intercaler dans le tableau des formes d'autres zones ou d'autres pays.

M. Bolus, dans une des lettres qu'il a bien voulu nous écrire avec cette bonne grâce clıarmante qui rend ses communications doublement intéressantes, insiste sur quelques autres caractères très particulier's à l'espèce, - ainsi sur le large réceptacle-plan qui est tronqué à la base et sur les akènes, très profondément striés et canaliculés, qui se trouvent de la sorte plus profondément lobés que l'auteur n’a pu l'observer dans aucune autre espèce.

Le S. sociorum posséderait, d'après les notes de son auteur, le port du $S$. crenatus Thunb : l'inflorescence et les capitules permettent toutefois une différenciation rapide.

La plante a été trouvée abondamment dans sa station, mais ne semble pas avoir été vue ailleurs.

F1G. 8. - Androcée fendu et étalé (8/1).

Fra. 9. - Articulation du style sur l'ovaire (schematisi).

Fig. 10. - Style et stigmate (9/1).

FIG. 11. - Soie du sommet de l'akène, fortement grossie. 


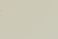

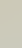

P.

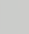

(1)

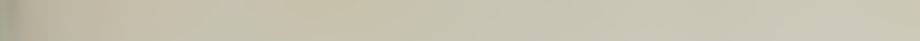





\section{PLANTA NOVA VEL MINUS COGNIT $Æ$}

EX IIERBARTO IIORTI THENENSIS

\section{PLANTES NOUVELLES OU PEU CONNUES}

CONTENUES DANS L'HERBIER

DE

L'HORTUS THENENSIS

\section{AVEC LES DESCRIPTIONS OU ANNOTATIONS}

DE

M. Ém. DE WILDEMAN

DOCTEUR EN SCIENCES

CONSERVATEUR AU JARDIN BOTANIQUE DE L'ÉTAT

Septième livraison (et Table). (MARS 1907)

VEUVE MONNOM

IMPRIMFUR ET FDITHUR

32, RUE DE L'INDUSTRIE, 32

BRUXELLES

Se vend exclusivement à l'Administration des biens et affaires de M. van den Bossche, 5, Grande Montagne, à Tirlemont. 



\section{PLANT E NOV E}

VEL MINUS COGNITA

EX HERBARIO HORTI THENENSIS 



\section{PLANTA NOVÆ VEL MINUS COGNIT}

EX HERBARIO IORTI THENENSIS

\section{PLANTES NOUVELLES OU PEU CONNUES}

CONTENUES IDANS L'HERBIER

$\mathrm{DE}$

L'HORTUS THENENSIS

\section{AVEC LES DESCRIPTIONS OU ANNOTATIONS}

DE

M. Ém. DE WILDEMAN

DOCTEUR LN SCIENGES

CONSERVATLLR AU JARDIN BOTANIQUE DE L'ETAT

\section{TOME I}

(1904-1907)

PLANCHES I A LVI

VEUTE MONNOM

IMPRIMEUIR HT HDITHUR

32, RUE DE l'indUSTRIe, 32

BRUXELLES 



\section{SEPTIÉME LIVRAISON}

Cette livraison contient des éturles sur sept espèces ou variétés de la famille des Moracées, dont quatre sont nouvelles. Les matérianx qui ont servi à sa préparation, ont été, sauf pour une variété du Dorstenia psilurus récoltée au Mozambique, empruntés à une série de plantes, qui nous a encore été célée par M. Ėlouard Luja et qui provient des lorèts du Sankuru, dans le district dı Kasai (État Indépendant du Congo).

Les espèces figurées sont les suivantes:

Dorstenia Luja Ise IVild. sp. nov.

Dorstenia Laurentii De Wild. var. Grevipedicellata De Wild. var. nov.

Dorstenia scaphigera Bureau.

Dorstenia psilurus Welw.

Dorstenia psilumes virr. compacta De IVild. var. nov.

Trymatococcus Kamerunianus Engler.

Bosqueiopsis Lujee De IVild. sp. nov.

Nous terminons avec cette septième livraison, le premier volume des Plante noxce rel minus cognita, qui comptera ainsi un peu plus de 240 prages de texte avec 56 planches.

L. B. 



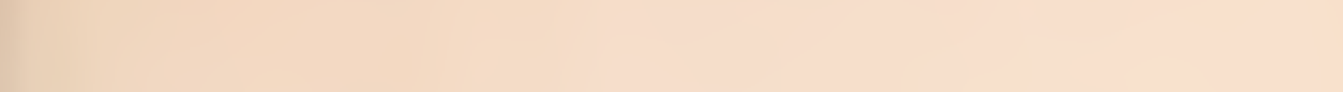
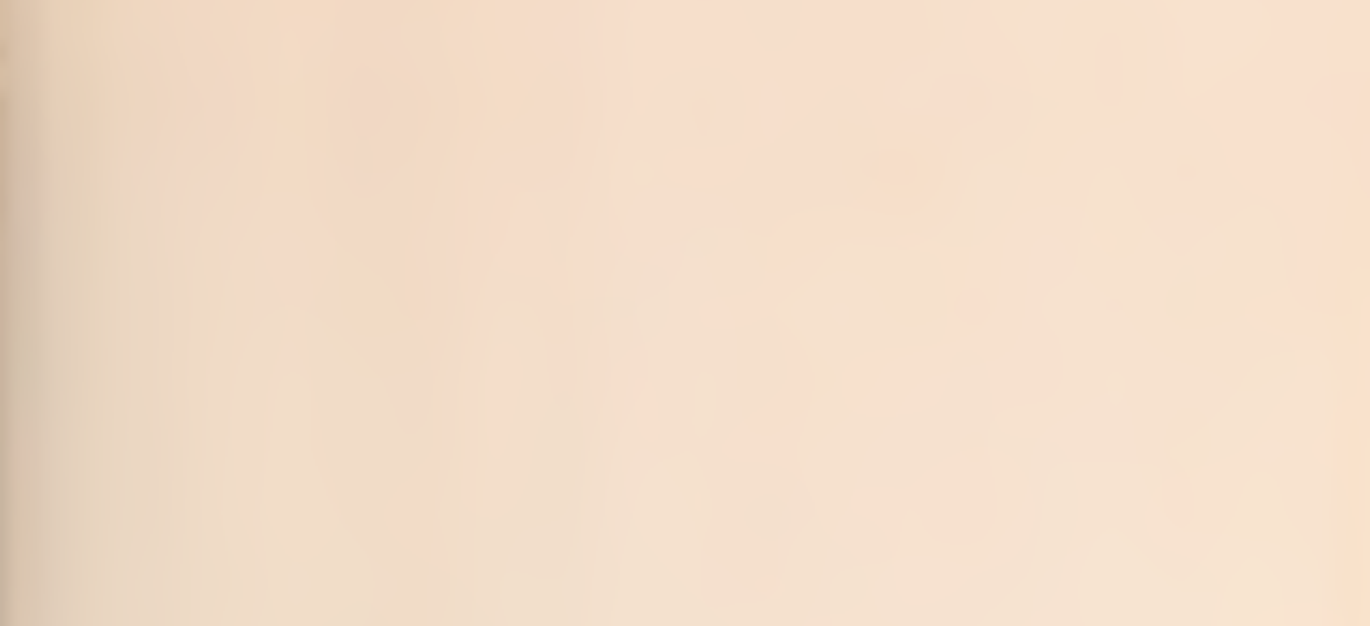

.
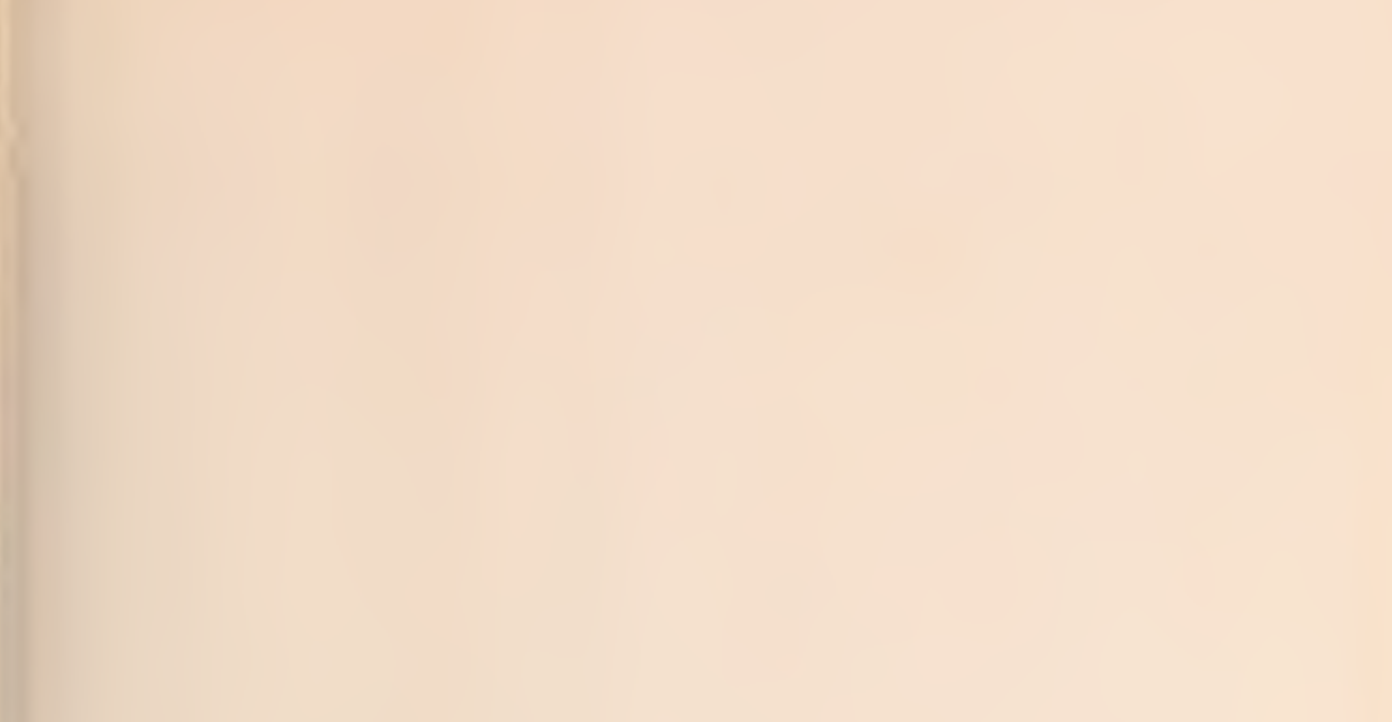


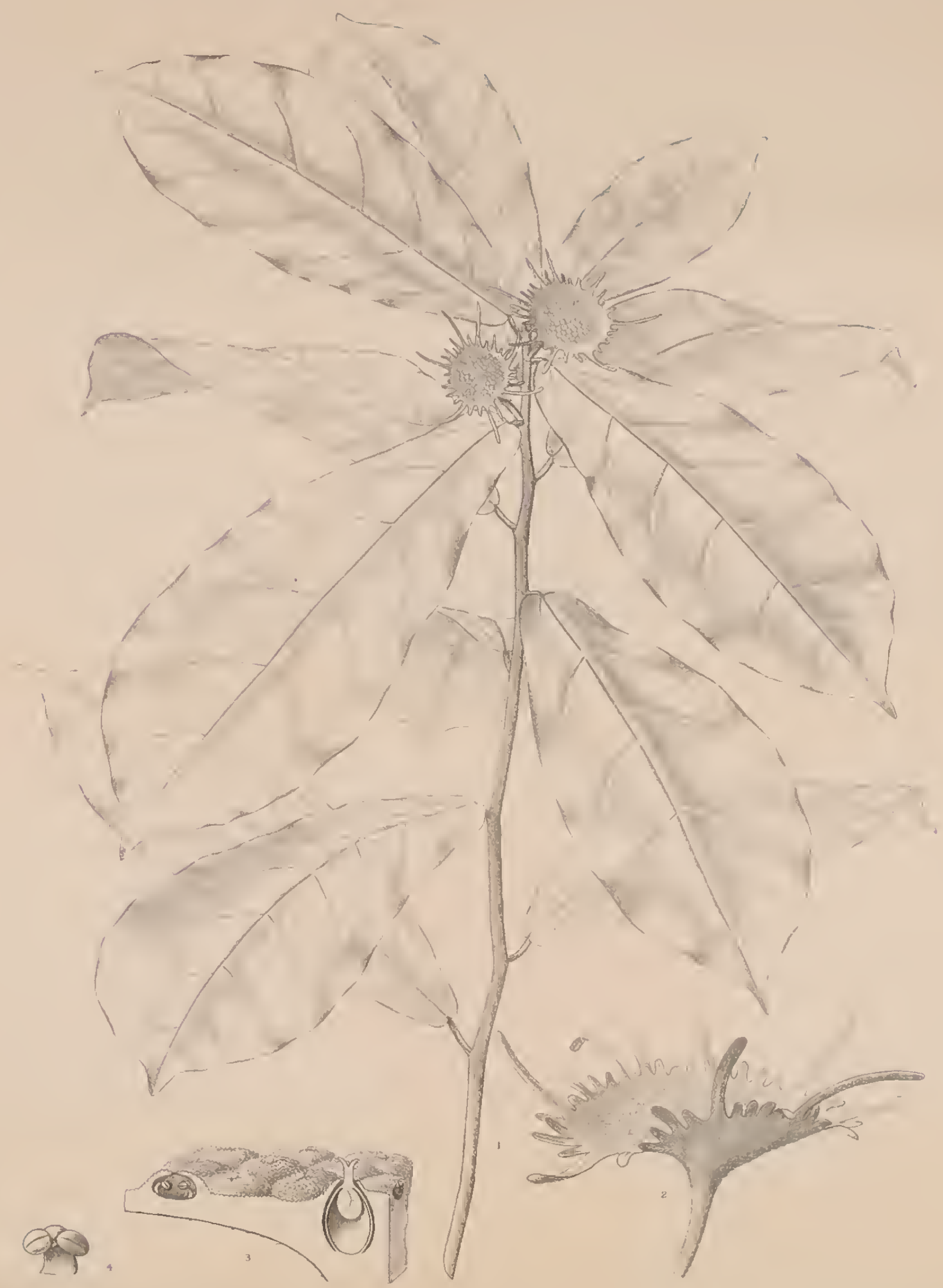




\section{Dorstenia Lujae De Wild. nov. sp.}

Dorstenia Lujae; caule atque foliorum petiolis brevissime et dense pilosis; stipulis ....; foliorum petiolo supra leviter canaliculato quam lamina multoties breviori, circ. $7-12 \mathrm{~mm}$ longo, lamina utrinque glabra, subtus flavo-viridi, obovato-lanceolata, ad basin obtusiuscula, subcordata, apice brevitel acuminata, margine leviter undulata, circ. $85-16 \mathrm{~cm}$. longa et $3,5-6,5 \mathrm{~cm}$. lata; ncrvis lateralibus I utrinque $7-8$ arcuatim subpatentibus ; inflorescentiis in axillis solitariis; pedunculo breviter , piloso, quam petiolus subequilongo vel longiore, usque $1,5 \mathrm{~cm}$. longo; receptaculo polygonali, 17 millim. lato, margine usque $3 \mathrm{~mm}$. lato, in bracteas circ. 30 a basi triangulari lineares valde inaequales exeunte cincto, bracteis usque $11 \mathrm{~mm}$. longis; floribus distinctis, masculis 2-andris perigonio subintegro instructis, florum femineorum perigonio integro stylum apice bifidum orarii immersi cingente.

'Tige et pétioles courtement mais densément velus, scabres; stipules. .; feuilles à pétiole légèrement canaliculé sur la face supérieure, beaucoup plus court que la lame, de 7 à $12 \mathrm{~mm}$. de long; limbe glabre sur les deux faces, plus pâle en dessous qu'au-dessus, obovale-lancéolé, rétréci ver's la base, celle-ci subcordée, courtement acuminé au sommet, légèrement ondulé sur les bords, de 8,5 à $16 \mathrm{~cm}$. de long et 3,5 à $6,5 \mathrm{~cm}$. de large; nervures latérales au nombre de 7 à 8 de chaque côté de la nervure

\section{Explication des figures de la planche $\mathrm{L}$.}

Fig 1. Rameau fleuri (2/1).

FIG. 2. - Inflorescence $3 / 1$ plus ou moins schématisée; le bord antérieur a éte repliè pour montrer la villosité externe du réceptacle. 
Inédiane, s'anastomosant en arc avant d'atteindre les bords, assez étalées, sauf l'inférieure qui forme avec la nervure médiane un angle aigu. Inflorescences vertes, solitaires dans les aisselles des feuilles supérie:ıres, à pédoncule courtement velu, environ aussi long ou un peu plus long que le pétiole atteignant $15 \mathrm{~mm}$. de long. Réceptacle irrégulièrement polygonal, de 16 à $17 \mathrm{~mm}$. environ de large, non compris les bractées, bord atteignant $3 \mathrm{~mm}$. de large, bractées au nombre de 30 environ, à base plus ou moins triangulaire, linéaires, inégales, atteignant jusque $11 \mathrm{~mm}$. de long. Fleurs màles et fleur's femelles distiuctes, les mâles à deux étamines, à périgone subentier, fleurs femelles à périgone entier, à style bifide au sommet, à ovaire immergé dans le réceptacle.

\section{Hab. - Forêts du Sankuru, mars 1904 (Éd. Luja, n. 177).}

Observations. - Nous considérons la plante figurée dans les dessins de la planche précédente comme une espèce nouvelle que nous dédions à soll collecteur M. Éd. Luja.

Par l'ensemble de ses caractères, le $D$. Luiae appartient à la section Eudorstenia telle que l'a décrite M. le prof. Engler dans sa monographie des Moraccae africanae, toutes les espèces de cette section, sauf le D. vivipara Welw. possédant un style à stigmates plus longs qu'épais, ce qui est le cas dans notre plante également; ces espèces se trouvent dès lors classées en trois groupes basés sur la forme du réceptacle : ovale ou étoilé, - allongé, c’est-à-dire au moins deux fois aussi long que large, très allongé.

C'est naturellement dans le premier des trois sous-groupes que nous devons chercher à intercaler notres plante. Ici encore nous trouvons deux subdivisions :

Bractées du bord du réceptacle très inégales.

Bractées du bord du réceptacle peu inégales.

Un coup d'œil jeté sur la figure I de notre planche démontre que le D. Lujae appartient au premier des deux groupements.

Fig. 3. - Coupe longitudinale à travers le disque de l'inflorescence passant par une fleur mâle et par une fleur femelle 15/1.

F1G. 4. - Étamine isolée 50/1. 
Mais les earacteres sur lesquels M. le prof. Fngler se basc, dans sa monographic, pour differencicr les espèces appartenant à ce groupement sont plus difficiles à apprécicr, ear ce sont des questions de plus ou moins. En effet il décrit les caractères de son groupe :

1) Réceptacle privé de bordure ou à bordure très étroite entr'c les bractécs.

2) Réceptacle à bord large, atteignant plus de la moitić du diamètr'e du réceptacle.

Certes ces caractères paraissent à premic̀re vuc très tranchés, et cependant nous nous ilemandons dans lequel des deux groupements nous classcrons la plante que nous figurons? Le rćceptacle possède un bor'd très accusé, mais ce bord est loin d'attcindre en largeur la moitié du diamètre de la partic centralc du réceptacle. Le $D$. Lujae constitue donc a notre avis une forme intermédiaire entre les types de ces deux groupes ct on pourrait considérer le $D$. Lujae, par l'aspect du réceptacle. comme une réduction du $D$ multiraxiata Engler.

C'est donc dans le petit groupement comprenant D. Barteri Burcau, ct $D$. multiradiata Engler que nous intercalerons le $D$. Lujae.

Nous modifierons dès lor's de la manière suivante le tableau analytique proposé par II. le prof. Englcr' :

† Réceptacle elliptique ou polygonal.

a) Réceptacle entouré d'environ 15 bractées inégales. . . . D. Barteri Bureau.

b) Réceptale entouré d'environ 30 bractées inégales. . .

1) Partie médiane du réccptacle de $3,5 \mathrm{~cm}$. cnviron de large, bord de $5-8 \mathrm{~mm}$. de large .

¿) Partie médiane du réceptacle de $1,2-1,3 \mathrm{~cm}$. de large, bord de 2-3 mm. cnviron de large $D$. Lujae De Wild.

D'autres caractères permettent encore la différenciation : citons la forme de la base des feuilles de notre $D$. Lujae, qui est très caractéristique, puisque les feuilles cordées à la base ne sont guère répandues dans le genre Dorstenia.

Depuis que nous avions trouvé cette espèce dans l'herbier Luja ct que nous l'avons décrite en manuscrit, nous avons eu l'occasion de la voir fleurir dans les serres du Jardin botanique de l'État à Bruxelles où les fleurs sc succèdent presque sans interruption. Le $D$. multiradiata Engler cst cultivé dans les serres du Jardin botanique de Berlin où il fleurit également pendant tout le courant de l'anncee. 



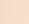




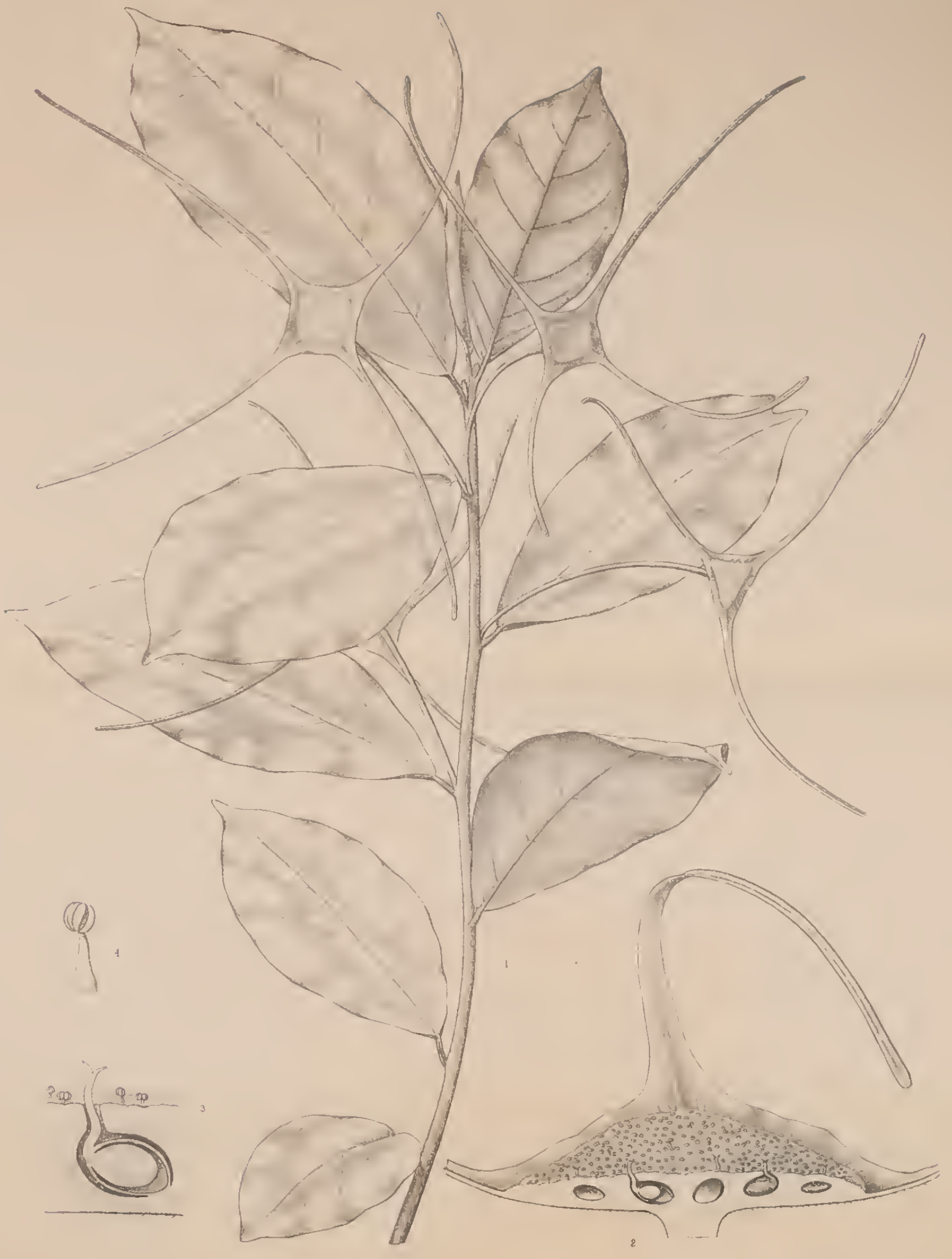




\section{Dorstenia Laurentii De Wild. var. brevipedicellata De Wild. nov. var.}

Dorstenia Laurentii var. brevipedicellata; erecta, ramulis et petiolis dense breviter pilosis; foliis breviter petiolatis, peíiolo 4-6 mm. longo, oblongis, basi cuneatis subcordatis, apice acuminatis obtusiusculis, integris, $4-11 \mathrm{~cm}$. longis et 2,5-6,5 cm. latis, nervis lateralibus utrinque circ. 7-8 arcuatim patentibus; inflorescentiis in axillis solitariis vel geminis; pedunculo quam petiolus cire. 10 plo longiore ; receptaculo 3-4-angulari, circ. $15 \mathrm{~mm}$. lato, margine usque 2,5 mm. lato, in bracteas 3-1 anguste lineares apiceus sub obtusum, et usque $7 \mathrm{~cm}$. longum; floribus masculis diandris; floribus femineis per totum receptaculum dispersis; ovario in stylum bifidum attenuato.

Plante à tiges dressées, à rameaux et pétioles densément mais courtement velus. Feuilles courtement pétiolées, à pétiole de 4 à $6 \mathrm{~mm}$. de long, à limbe oblong, rétréci-cunéifor'me vers la base, celle-ci légèrement subcordée, courtement acuniné au sommet, à acumen obtus, entier sur le bord, glabre sur les deux faces, de 4 à $11 \mathrm{~cm}$. de long, et de 2,5 à $6,5 \mathrm{~cm}$. de large, à nervures latérules de premier ordre au nombre de 7 à 8 , anastomosées en ar'c vers le bord, étalées-dressées, en creux sur la face supérieure, légèrement en relief sur la face inférieure. Inflorescences glabres ou glabrescentes, axillaires, solitaires ou géminées, à pédoncule environ dix fois aussi long que le

\section{Explication des figures de la planche LI.}

Fig. 1. - Tige fleurie (1/1).

Fia. 2. - Coupe transversale de l'inflorescence (6/1). 
pétiole, à réceptacle triangulaire ou quadrangulaire, atteignant environ $15 \mathrm{~mm}$. de diamètre, à partie médiane d'un brun noirâtre, à bord mince atteignant $2,5 \mathrm{~mm}$. de large, se terminant aux angles en bractées étroitement linéaires, subobtuses au sommet et atteignant jusque $7 \mathrm{~cm}$. de long. Fleurs mâles à deux étamines; fleurs femelles distribuées sur toute la surface du réceptacle; ovaire immergé dans le disque, à style exsert, divisé au sommet en deux stigmates relativement courts et étalés.

Hab. - Forêts du Sankuru, juin 1903 (Éd. Luja n. 54).

Observations. - Nous considérons cette plante comme une variété de la plante qu'Ém. Laurent avait collectée dans la mème région, aux environs de Kondue, le 25 novembre 1903 et que nous arons décrite dans le fasc. I de la “ Mission Émile Laurent" (p. 68 pl. XXXII).

Comme nous le faisions ressortir en décrivant le type. la plante parait voisine des D. intermedia Engler et Ophiocoma K. Schum. et Engler; mais cependant certains caractères séparent bien nettement notre plante des deux types cités et figurés dans la monographie des Moraceae africana, p. 17 pl. IV D et IV C., entre autres, la grandeur relativement considérable du bord qui entoure la partie florifère du réceptacle et qui rappelle par suite le D. subtriangularis Engler; de plus chez cette dernière espèce comme chez toutes ctlles qui appartiennent à la mème subdivision, le disque est muni de nombreuses bractées marginales irrégulières.

Dans les autres espèces que nous avons citées, $D$. intermediaet ophiocoma, chez lesquelles il n'existe pas de bord, les bractées sont toujours plus nombreuses.

Les caractères qui différencient notre variété du type pour la définition duquel nous n'avons eu, il est vrai, qu'un échantillon, résident dans la grandeur des feuilles et dans la longueur des pédoncules floraux notablement plus réduits, puisque chez le type ils atteignent 9 centimètres de long.

Le $D$. Laurentii et sa variété brevipedicellata sont particulièrement intéressants et paraissent localisés dans la région du Sankuru; nous n'en avons pas reçu d'exemplaires d'autres régions congolaises.

Fig. 3. - Fragment de celle coupe 15/1.

FIG. 4. - Élamine isolee (18/1). 
,

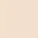




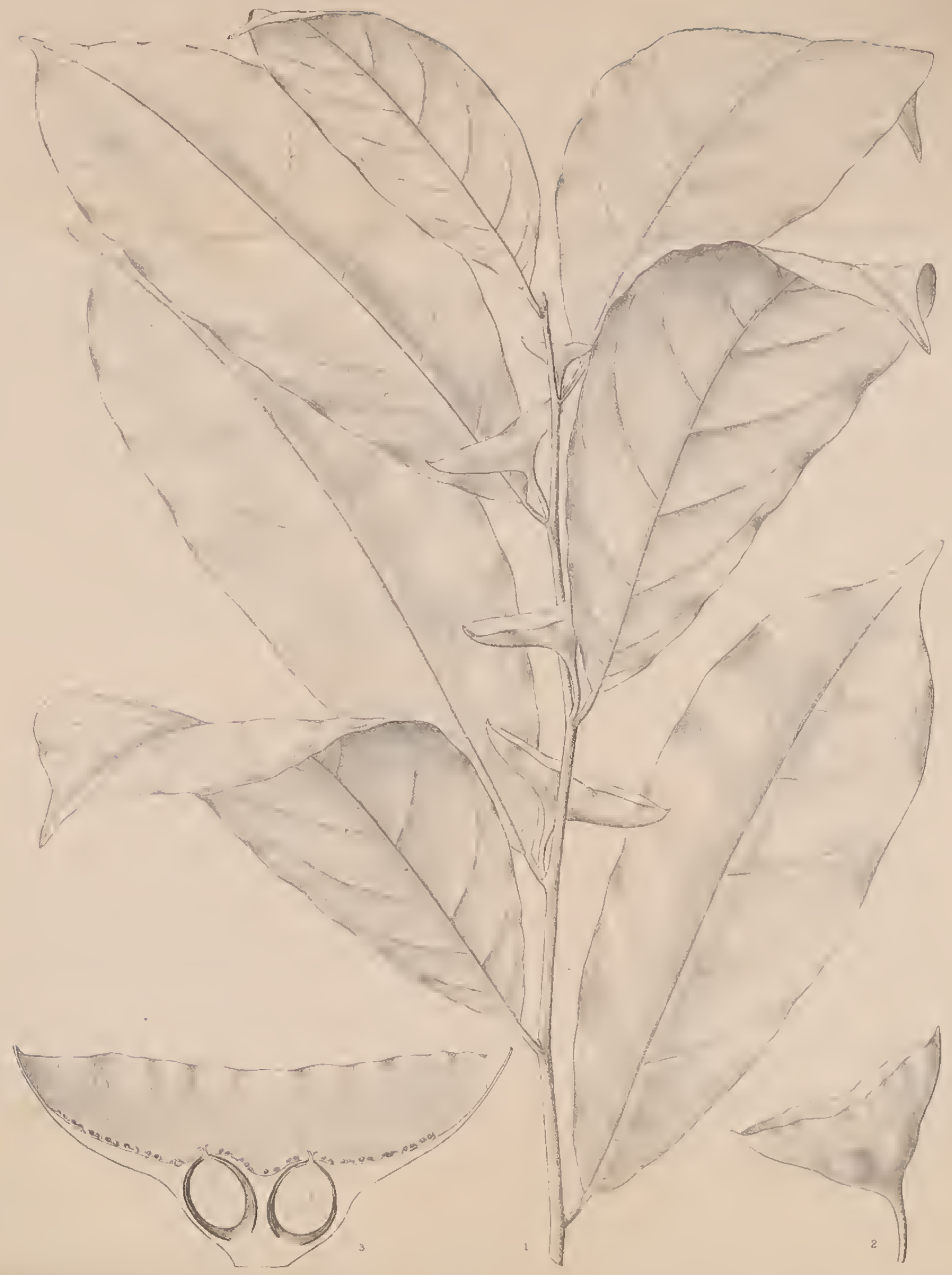




\section{Dorstenia scaphigera Bureau}

in Bulletin du Muésum d'Hist. nat. Paris I (1895) p. 60; A. Engler' Monog. Afrik. Pllanzen I Moraceae africanae p. 19.

Plante constituant un petit buisson, ¿i tiges dressćes atteignant $1 \mathrm{~m}$. de haut, pubescentes à l'état jeune, devenant rapidement glabres. Stipules séticées de 2 mu, environ de long', rapidement caduques. Feuilles à pétiole court, de 5 ¿ $10 \mathrm{~mm}$. de long, légèrement canaliculé supéricurement; limbe membraneux, obovale-elliptique ou elliptique, cunéiforme à la base, acuminé au sommet, à acumen subobtus souvent mucroné, à bords irrégulièrement sinués, de 5,5 à $17 \mathrm{~cm}$. de $\operatorname{loug}$ et 2,5 à $8 \mathrm{~cm}$. le large; nervures latérales primaires au nombre de 6 à y de chaque côté de la nervure médiane, s'anastomosant irrégulièrement ver's le bord, plus proéninentes en dessous qu'au-dessus. Inflorescences axillaires, solitaires, naviculoïdes, à pédoncule grèle plus lonģ (pue le pétiole, de 1 à $1,5 \mathrm{~cm}$. de long. Réceptacle étroit, relativement pauciflore, à bor latéral élargi, sinué, de 3 à $4,5 \mathrm{~cm}$. de long, rebord dressé de 5 à $6 \mathrm{~mm}$. de lıaut; fleurs mâles nombreuses, ¿̀ 2 étamines entourées par le périanthe obscurément bilobé, fleurs femelles au nombre de 2 ou 3 sur une ligne médiane, à ovaire profondément immergé, ovoïde, terminé par un style exsert, bifide.

\section{Explication des figures de la planche LII.}

Fig. 1. - Rameau fleuri (1/1).

FiG. 2. - Inflorescence avec deux graines, vue de profil (1/1).

Fro. 3. - Coupe longitudinale de l'infrutescence (2/1). 
Hab. -- Kondue (Sankuru) et environs, avril-août 1903 (Éd. Luja, n. $1,32,81$ ).

Découvert dans le Haut-Kemo (Congo français) par M. J. Dybowski et assez répandu dans diverses régions de l'État du Congo tant au sud qu'au nord de l'Équateur.

Observations. - C'est sur un échantillon recueilli par M. J. Dybowski, directen' du Jardin colonial de Nogent-sur'Marne, que M. Bureau, l'ancien professeur de la chaire de systématique au Muséum d'Histoire naturelle de Paris, a décrit cette plante.

Elle appartient au sous-genre Eudorstenia tel que le comprend M. le prof. Engler dans sa monographie des Moraceae africanae, à lauquelle nous renvoyons dans la notice bibliographique citée en tête de cet article. En effet, les réceptacles ne possèdent pas de bractée entre le bord membraneux et le point d'attache au sommet du pédicelle; en outre le style est divisé en deux lobes.

Dans cette section des Eudorstenia, qui renferme le plus grand nombre des espèces africaines, $M$. le prof. Engler' se base pour classer les espèces sur les proportions du style, plusieur's fois plus long qu'épais ou légèrement plus long qu'épais. Dans ce dernier groupement il n'a pu classer qu'une seule espèce le $D$. vivipara Welw. Les trois autres groupes se basent sur la forme du réceptacle : $1^{\circ}$ en bouclier ou en étoile; $2^{\circ}$ allongé, au minimum deux fois aussi long que large, mais à bractées latérales et terminales; $3^{\circ}$ très allongé, à bords latéraux indivis.

Un coup d'œil jeté sur notre planche fait voir que c'est dans cette dernière division que nous devons chercher le D. scaphigera. Nous y trouverons également le $D$ psilurus Welw., sur lequel nous reviendrons plus loin et les $D$. bicornis Schweinf. et scabra Bureau, mais chez toutes ces dernières espèces, lo pédicelle du réceptacle se trouve disposé plus près d'une des extrémités et l'une de ces extrémités ou les deux sont munies d'un prolongement plus ou moins accentué, ce qui n'est pas le cas chez le $D$. scaphigera, comme le montrent d'ailleurs fort bien les figures de notre planche.

Jusqu'à ce jour, le $D$. scaphigera est la seule espèce du genre Dorstenia dont le réceptacle présente cette forme caractéristique de baguette qui lui a fait donner son nom.

Nous ne pensons pas que cette espèce se trouve actuellement dans les collections de plantes vivantes d'un jardin botanique; elle mériterait cependant d'être introduite dans les cultures pour la singularité de ses inflorescences. 
PL Vuvae Herb Hort THENENSIS

PLLIII

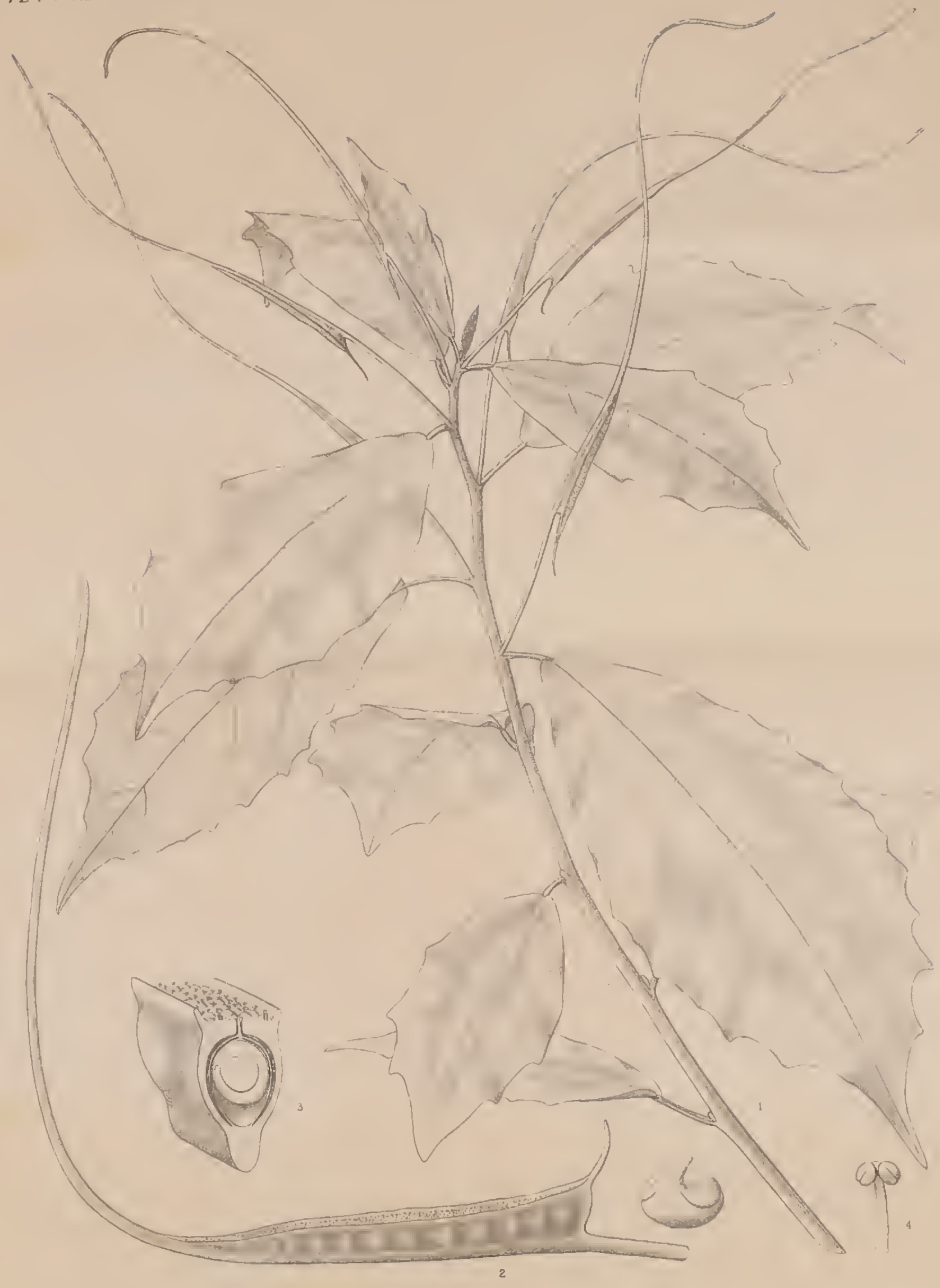

A dipreval ad nat del

Etabits Minot Faris 


\section{Dorstenia psilurus Welw.}

in Transact. Linn. Soc. XXVII, 1 (1869) p. 71 ; Bureau in DC. Prodr. regn. veget. XVII p. 272; Engler Monog. Afrik. Pflanzen I Moraceae africanae p. 20.

Rhizome cylindrique, rampant, noueux, charnu; des nouds partent des tiges dressées de $70 \mathrm{~cm}$. de long, non ramifiées, légèrement charnues, devenant glabres à la base, lâchement feuillues dans leur partie inférieure, plus densément feuillues vers le sommet et pubescentes. Stipules petites, linéaires-lancéolées, caduques. Feuilles à pétiole beaucoup plus court que la lame, de 5 à $15 \mathrm{~mm}$. de long; limbe à poils épars, blanchâtres, ou glabrescent sur la face supérieure, plus pâle en dessous qu'au-dessus, glabre sur la face inférieure avec quelques poils épars sur les nervures, ovale-elliptique ou obovale, cunéiforme à la base, plus ou moins brusquement acuminé au sommet, largement denté sur les bords, rarement entier, de 3 à $6 \mathrm{~cm}$. de large et 6 à $15 \mathrm{~cm}$. de long. Inflorescence solitaire à l'aisselle des feuilles, dressée, pédonculée, à pédoncule de 2,5 à $4,5 \mathrm{~cm}$. de long, réceptacle lancéolé, linéaire, de 2 à $3 \mathrm{~cm}$. de long, terminé à la base en une sorte d'apicule et au sommet en un long prolongement filiforme, atteignant jusque $10 \mathrm{~cm}$. de long. Fleurs mâles nom-

\section{Explication des figures de la planche LIII.}

Fig. 1. - Rameau avec feuilles et fleurs (1/1).

Fia. 2. - Inflorescence $(2,5 / 1)$. 
breuses, à une seule étamine à deux loges subglobuleuses divergentes, à périanthe irrégulièrement denté ; fleurs femelles moins nombreuses disposées dans l'axe du réceptacle, enfoncées dans le tissu, à style à deux branches dépassant légèrement le périgone.

Hab. Forêt du Sankuru, 25 août 1903 (Éd. Luja, n. 82).

Cette espẻce existe dans d'autres régions de l'État Indépendant du Congo et dans l'Angola où elle a été trouvée par Welwitsch.

Observations. - Le Dorstenia psilurus découvert et decrit par Welwitsch appartient, d'après les données de la monographie de M. le prof. Englel, que nous avons citée dans la bibliographie réservée en tête de cet article, à la section Eudorstenia Engler. Cette section se caractérise par le style divisé en deux stigmates et par l'absence de bractée entre le bord et la base du réceptacle.

La forme très particulièl'e du l'éceptacle de cette espèce permet de la différencier très facilement de la plupart des autres espèces du genre. Elle apjartient cependant au mème sous-groupe que le $D$ scaphigera Bureau que nous avons étudié plus haut, mais chez ce dernier le pétiole se trouve, comme nous l'avons dit, au milieu du r'éceptacle. Chez le D. bicornis Schweinf. le même caractère se représente mais il est parfois moins net, et il y a déjà une tendance à la prédominance d'une des parti s du réceptacle sur l'autre; d'ailleurs, chez ce $D$. bicornis, que l'on connait uniquement de la région des Niams-Niams, le réceptacle est disposé en croissant, tandis que daus les plantes que nous figurons il est très allongé et le pédicelle floral s'insère tout à fait à la base du réceptacle. Ce dernier caractère exi-te également chez le D. scabra, qui a beaucoup d'analogie avec notre plante, mais s'en différencie pas l'absence de dents ou par des dents très réduites au bord du limbe.

Le D. psilurus Welw. paraitt répandu dans plusieur's régions congolaises; nous avons eu l'occasion d'en voir de nómbreux échantillons. La plante semble très variable : elle peut en tous cas, comme le montrent les domnées de notre description, acquérir dans toutes ses parties des proportions beaucoup plus considérables que celles indiquées dans la diagnose princeps et dans la monographie du plof. Engler.

FIG. 3. - Coupe transversale de l'inflorescence, avec fruits non complètement mûrs $(8 / 1)$.

FIG. 4. - Étamine (60/1). 
Un caractère sur lequel nous tenons à insister, c'est celui de la présence, chez tous les échantillons du Congo qui nous sont passés entre les mains, de tiges dressés simples non ramifiées ou présentant parfois, mais rarement, ver's la base, des rameaux courts et avortés.

Le $D$. psilurus existe à l'état vivant dans les serres du Jardin botanique de l'État; sa cultur'e est très aisée et on l'y voit constamment fleurir. 


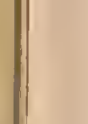


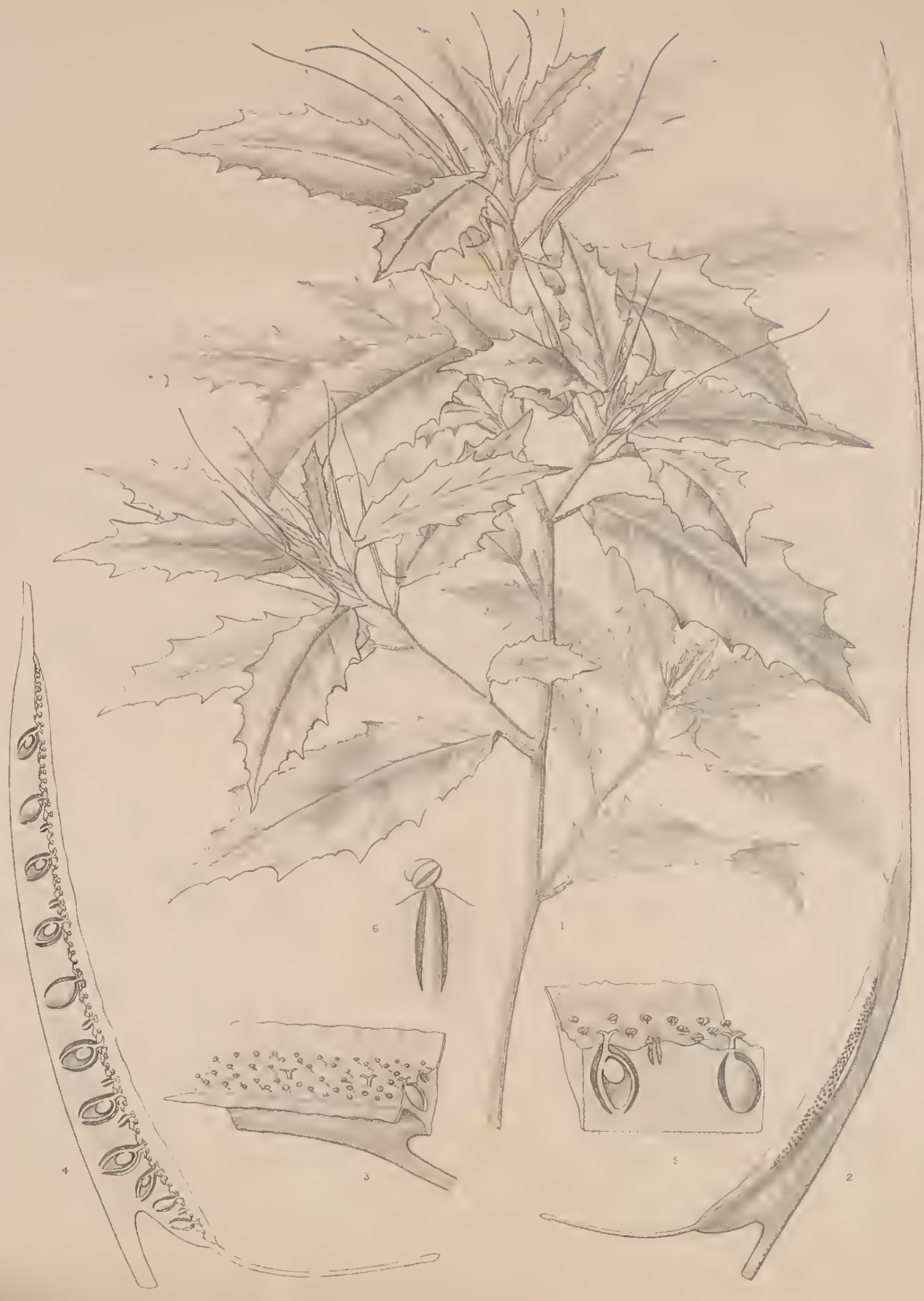

A d Apreval ad nat del

Etabl ${ }^{\text {ts }}$ Minut Pars 
Dorstenia psilurus var. compacta De Wild. nov. var.

Dorstenia psilurus var. compacta; caule erecto, dense ramoso, $40 \mathrm{~cm}$. circ. alto, inferne glabriusculo et laxius apicem versus densius foliato et pubescente; foliorum petiolo quam lamina 5-9 plo breviore, 7-15 mm. longo; lamina tenui, supra glabriuscula, subtus pallidiore et nervis plus minus dense pilosa, ovato-elliptica, acuminata, acuta, margine dentata, dentibus acutis ; inflorescentia axillaria solitaria, $5-15 \mathrm{~mm}$. pedunculata, erecta, lanceolato-linearis, $2-2,5 \mathrm{~cm}$. longa, apice in bracteam circ $3 \mathrm{~cm}$. longam linearem receptaculum superantem, basi in breviorem exeunte.

Tiges dressées, réunies en touffes plus ou moins compactes, ramifiées plus oủ moins densément, atteignant $40 \mathrm{~cm}$. de haut, glabrescentes, à feuilles espacées, ou nues à la base, densément feuillues et pubescentes vers le sommet; feuilles à pétiole beaucoup plus court que le limbe, de 7 à $15 \mathrm{~mm}$. environ de long; limbe mince, glabrescent sur la face supérieure, plus pâle en dessous qu'au-dessus, à nervures plus ou moins densément velues, ovales-elliptiques, acuminées, aiguës, dentées ou doublement dentées sur les bords, à dents primaires aiguës, inflorescences axillaires, solitaires, à réceptacle pédonculé, dressé, lancéolélinéaire, de 2 à $2,5 \mathrm{~cm}$. de long, à bractée terminale

\section{Explication des figures de la planche LIV.}

Fta. 1. - Fragment de lige florifere ramifiée (1/1).

Fia. 2. - Inflorescence (5/1).

Fia. 3. - Fragment de l'inflorescence (12/1).

F1a. 4. - Coupe lougitudinale de l'inflorescence (8/1). 
atteignant $3 \mathrm{~cm}$. de long, bractée basilaire beaucoup plus courte, pédicelle de 5 à $15 \mathrm{~mm}$. de long.

Hab. - Mozambique : Morrumbala, dans les plantations de café, 10 janvier 1901 , n. 372 et mars 1901 , n, 382 (E்d. Luja).

Observations. - C'est à titre de variété que nous séparons cette plante du Morrumbala, du type $D$ psilurus du centre de l'Afrique. La planche, tout en étant de format déjà considérable, il ne nous a été possible de figurer qu'une des tiges des touffes constituant la plante.

C'est, comme nous le faisons ressortir dans la diagnose, par la ramification nombreuse et par les rameaux rapprochés, que la plante recueillie par M. Luja se différencie du type

Mais si l'on veut jeter un coup d'œil sur les deux planches on remarquera d'autres caractères qui permettent la séparation, au moins comme variété, des deux plantes figurées. Feuilles et inflorescences sont dans la plante de la planche ci-jointe beaucoup plus réduites que celles de la planche précédente; en outre la dentelure est plus accusée chez le D. psilurus var. compacta, elle peut même être considérée comme double : entre les grandes dents très aiguës se trouvent en général une ou plusieurs dents moins accusées mais cependant nettes.

Cette variété serait-elle due aux conditions du milieu? Dans la région où elle a été observée, la plante se trouvait à une certaine altitude.

C'est en tous cas une variété intéressante : elle est peut-être un des extrèmes de la série des formes sous lesquelles peut se présenter cette variable et curieuse espèce, le $D$. psilurus.

Fig. 5. - Fragment de la coupe longitudinale de l'inflorescence (15/1).

Fıa. 6. - Fleur mâle réduite à une étamine (60/1). 
. 


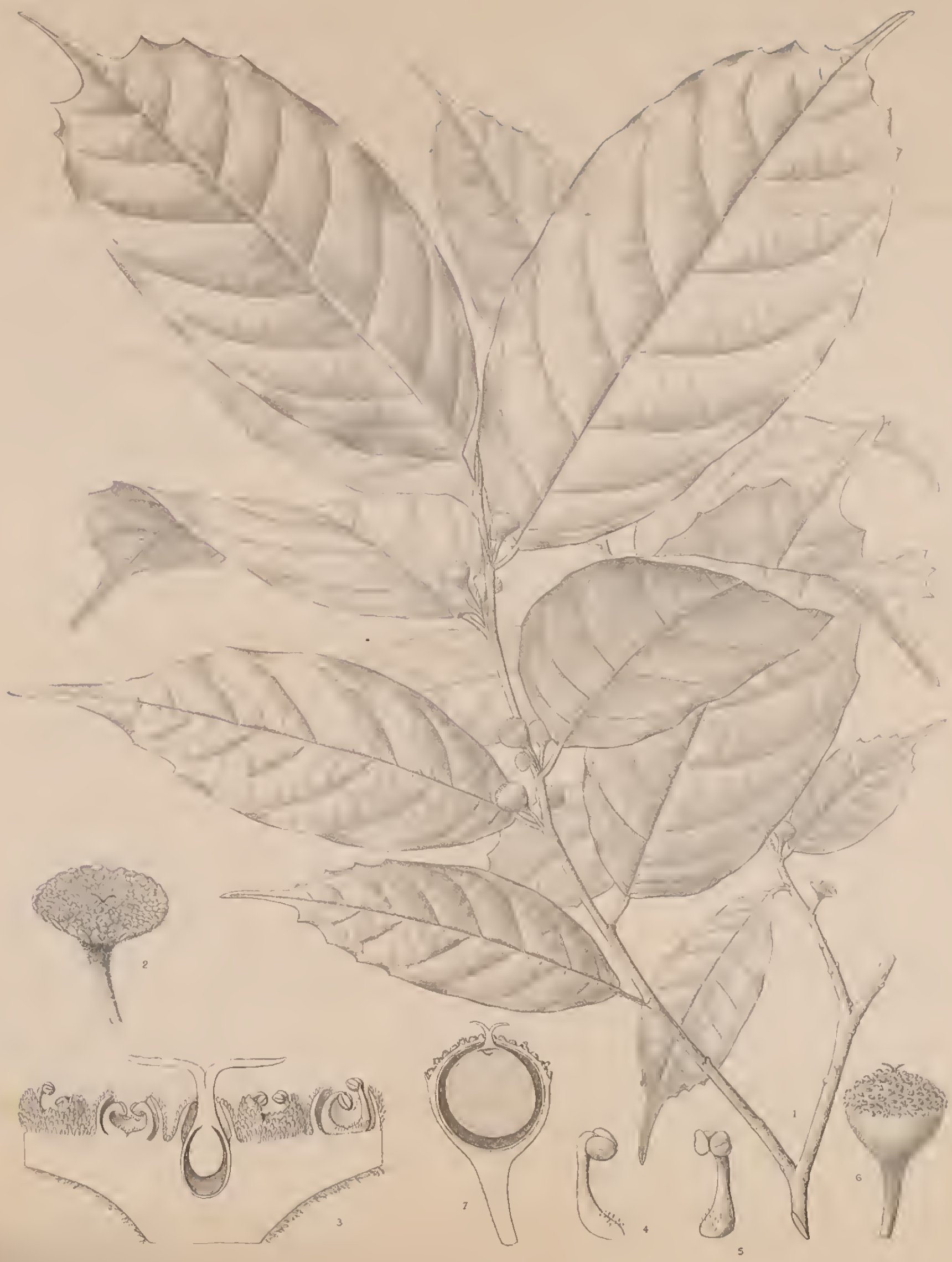




\section{Trymatococcus kamerunianus Engler}

Monogr'. Afrik. P(lanzen I Moraceae africanae (1898) p. 29 pl. XI fig. B.

Dorstenia kamerunianus Engler in Bot. Jahrb. XX (1894) p. 142.

Arbrisseau ou arbre atteignant $6 \mathrm{~m}$. de hateur, ramifié, à rameaux grêles, à écol'ce grisìtre, plus ou inoins verruculeuse, velus à l'état jeune. Stipules étroitement lancéolées, plus ou moins persistantes, de 4 à $8 \mathrm{~mm}$. de long. Fenilles courtement pétiolées, à pétiole de 5 is $8 \mathrm{~mm}$. de long, à limbe rigide, membraneux, plus ou moins brillant sur la face supérieure, gondolé entre les nervures, glabre sur cette face comme sur l'inlérieure mate, oblong ou oblong-elliptique, de 7 à $20 \mathrm{~cm}$. de long et 2 i $8 \mathrm{~cm}$. de large, subarrondi à la base, plus ou moins brusquement acuminé au sommet, à acumen atteignant près de $3 \mathrm{~cm}$. de long, obtus, irrégulièrement denté sur les bords au sommet, parfois presque entier; nervures latérales au nombre de 7 à 8 de chaque côté de la nervure médiane, en creux sur la face supérieure, en relief sur la face inférieure, anastomosées en arc près du bord de la feuille. Inflorescences axillitires, solitaires ou par 2 à 4 , de $7 \mathrm{~mm}$. environ

Explication des figures de la planche LV.

Fir. 1. - Rameau avec fleurs et fruits (1/1).

Frs. 2. - Inflorescence $(5 / 1)$.

Fıg. 3. - Coupe longitudinale d'une partie de l'inflorescence (32/1).

Fig. 4. Étamine vue de profil $(70 / 1)$. 
de diamètre, densément velues extérieurement, à pédoncule environ aussi long que le pétiole; réceptacle turbiné, à bord ondulé, garni de nombreuses bractées ciliées, fleurs mâles nombreuses, à périanthe velu, à 2 étamines, à filet plus ou moins recourbé, à deux anthères séparées par le filet; fleur femelle solitaire, immergée dans le disque, à style dépassant les fleurs mâles et divisé au sommet en deux stigmates linéaires, étalés ; réceptacle fructifère subglobuleux de $1 \mathrm{~cm}$. environ de long.

Hab. - Forêt du Sankuru, juin 1903 (Éd. Luja, n. 11).

Cette plante, qui existe dans d'autres régions congolaises, se rencontre au Kameroun et dans l'Angola.

Observations. - Le genre Trymatococcus créé par Poeppig et Endlicher, est un des plus intéressants de la famille des Moraceae. Comme le fait ressortir M. le professeur Engler dans la monographie des Moracées africaines, à laquelle nous avons renvoyé le lecteur daus la notice bibliographique, les espèces de ce genre sont non seulement intóressantes par leurs caracteres morphologiques mais encore par' leur distribution, puisque l'une d'elles, la plus anciennement connue, le $T$. amazonicus est de l'Amérique du Sud, tandis que les autres sont du continent africain.

La plante que nous avons décrite et figurée plus haut, l'avait déjà été dans la monographie du prof. Engler ; mais nous avons cru utile d'en donner quelques figures nouvelles, afin de bien montrer la variation des caractères que l'on croirait pouvoir tırer de la forme des limbes. Si l'on veut comparer la figure de notre planche avec la figure $\mathrm{B} a$ de la planche XI des Moraceae africanae, on remarquera que les feuilles figurées par M. le prof. Engler constituent la forme moyenne; elles sont obtusément cunéiformes à la base et relativement peu brusquement acuminées au sommet. Sur le rameau que nous avons choisi pour être figuré on peut voir vers la base des rameaux des feuilles cunéiformes à la base et rétrécies insensiblement en acumen au sommet; plus haut on en trouve d'autı'es arrondies à la base et l'une d'entre elles est même très brusque ment acuminée au sommet.

Cette forme caractéristique des feuilles nous avait fait croire au pre-

Fta. 5. - Étamine vue de face (70/1).

Fia. 6 - Jeune fruit, entier (3/1).

Fıa. 7. - Co ipe longitudinale du fruit (3/1). 
mier abord que cettc forme de Sankuru pouvait eonstituer une variété distinete, mais les nombreux matériaux qui nous sont passés entre les mains nous ont permis de voir toute une série de formes intermédiaires.

Un autre earactère sur lequel notr'e attention avait été égalcment attirée est le nombre d'infloreseences que l'on r'encontre à l'aisselle des feuilles; d'après la description princeps du prof. Engler', les inflorescenees sont solitaires ou géminées à l'aisselle des feuilles; - dans la forme du Sankuru, très vigoureuse, on en trouve parfois quatre à la mème aisselle, et sur le mème rameau, eomme le montre notre planche, on peut reneontrer des inflorescenees isolées.

M. le prof. Engler a décrit une variété Welwitschii earactérisée par les bords de la moitié supérieure du limbe foliaire inégalement et irrégulièrement dentés. Certaines des feuilles de notre plante paraissent présenter' ee earaetère. mais il est loin d'être eonstant dans nos éehantillons. Nous ne pouvons done rapporter la forme du Sankuru à celle de l'Angola, que nous ne eonnaissons d'ailleurs que par la courte diagnose qu'en a publiée M. le prof. Engler. Il pourrait fort bien se faire que cette var. Welwitschii ne soit qu'une des variations du type $T$. kamerunianus, qui parait, d'apres les matériaux que nous possédons, une plante très polymorphe.

Notons encore, pour faire voir le polymorphisme de ee type, que les échantillons r'ecueillis par M. Luja provenaient d'un arbre mesurant 6 mètres de haut, tandis que d'après M. Engler les plantes du Kameroun atteignaient au maximum 2 mètres de hauteur. 



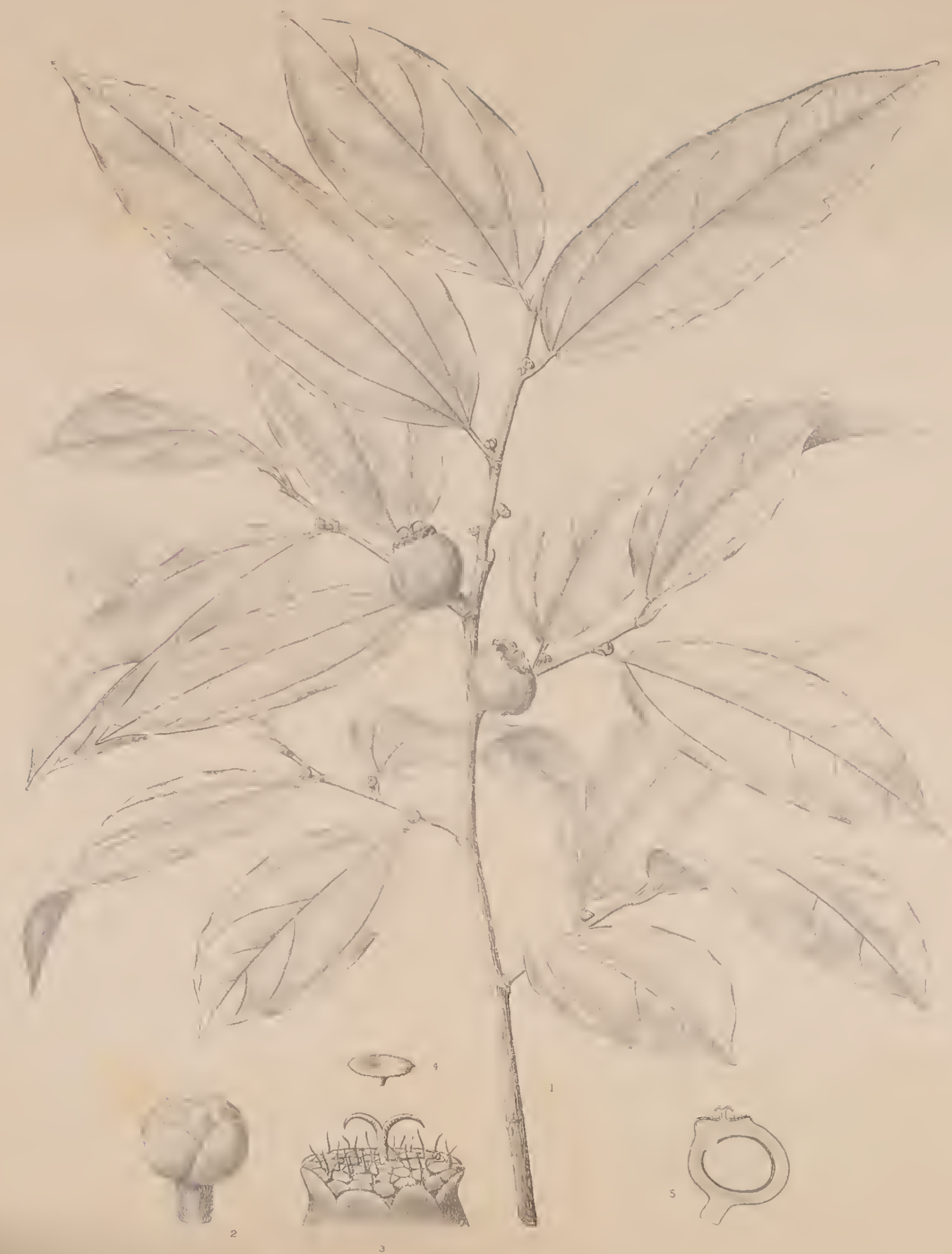




\section{Bosqueiopsis Lujae De Wild. nov. sp.}

Bosqueiopsis Lujae; arbor elatus; ramisteretibus juvenibus dense sed breviter pilosis, demum glabris cortice striato; foliis alternis, petiolatis, petiole sparse piloso, $\bar{\jmath}-9 \mathrm{~mm}$. longo, lamina elliptica, vel ovati basi rotundata, apice acuminato, acumine obtuso vel apiculato, 6,5-13 cm. longo et $2,5-5 \mathrm{~cm}$. lato, panlo inxquilaterialia; supra in sicco obscure brumea, infra pallidiora; nervis lateralibus utrinque circ. 4 , basilaribus et superioribus longe distantibus, ante marginum arcuatim anastomosantibus et cum venulis reticulatis supra paulo vel non, infra leviter prominentibus anastomosantibus. Inflorescentiis axillaribus breviter pedicellatis, pedicello $2-4 \mathrm{~mm}$. longo, pubescenti, receptaculo extus breviter pubescenti, floribus . ..... disco bracteis peltatis munito, bracteis ad marginem ciliatis, staminibus inter bracteas intermixtis, quam bractere longioribus; ovario infero sucentrali, stylo erecto, apice bilobato, ovulo solitario ; fructibus oblıquis, apice vestigiis bracteolarum staminumque coronatis, pubescentibus, circ. $16 \mathrm{~mm}$. latis.

Arbre élevé, à rameaux cylindriques velus à l'état jeune, devenant glabres, à écorce strièe-fendillée; feuilles alternes, caduques, réapparaissant aux premières pluies, pétiolées, à pétiole éparsement velu, de 5-9 $\mathrm{mm}$. de long, à limbe elliptique ou ovale, arrondi à la base, parfois légèrement inéquilatéral, acuminé au sommet, a acumen obtus ou courtement apiculé, de $6,5-13 \mathrm{~cm}$. de long et 2,5-5 cm. de large, d'un beau vert à l'état frais, d'un brun

\section{Explication des figures de la planche LVI.}

Frg 1. - Rameau avec fleurs et fruits (1/1).

FIG 2. - Bouton (7/1). 
foncé à l'état sec sur la face supérieure, plus pâle en dessous et plus mat; nervures latérales au nombre de 4 environ de chaque còté de la ligne médiane, la nervure basilaire s'étendant sur les deux tiers du limbe, très distante des nervures supérieures; nervures peu proéminentes sur les deux faces, mieux marquées sur la face inférieure que sur la face supérieure. Inflorescences axillaires, courtement pédicellées, à pédicelle velu de $2-4 \mathrm{~mm}$. de long, pubescent comme les tiges, réceptacle pubescent extérieurement, fleurs..., disque muni de bractées peltées, ciliées sur les bords, étamines logées entre les bractées que les filets dépassent; ovaire infère, style dressé, à stigmate bilobé; fruits obliques, courtement velus, grisâtres, couronnés par les vestiges des bractées et des étamines, de $16 \mathrm{~mm}$. environ de large.

Hab. - Forêts du Sankuru, 6 août 1903 (Éd. Luja, n. 78).

Observations - Nous avons créé le genre Bosqueiopsîs en 1901 dans les Plantae Gilletianae congolenses II (in Bull. de l'Herbier Boissier, sér. 2, n. 8. p. 840) pour une plante récoltée dans le Bas-Congo par le Frère J. Gillet, S. J.: nous avons dans cette note fait ressortir les caractères qui nous permettaicnt de faire de cette plante un genre nouveau : c'était surtout la présence de bractées peltées, que chez les Moraceae africanae on rencontrait seulement chez les espèces des genres Treculia et Musanga et que récemment M. le prof. Engler a observé chez une espèce d'un genre nouveau encore, le Sloetiopsis usambarensis Engl. (in Bot. Jahrb. XXIX, p. 573 c. fig.).

La plante qui nous a été rapportée du Sankuru par M. Éd. Luja, et que nous n'avons plus eu l'occasion de revoir dans d'autres envois de la région, constitue la deuxième espèce de notre genre Bosqueiopsis De Wild. et Th. Dur.; elle se distingue surtout par la forme des feuilles.

FIG. 3. - Sommet du fruit avec bractées peltees entre lesquelles dépassent les filets staminaux $(4 / 1)$.

FIo. 4. - Bractée peltée, isolée (13/1).

Fro. 5. - Coupe longitudinale du fruit (1/1). 
Chez le B. Gilletii les feuilles sont beaneoup plus petites, et attengnent au maximum $8 \mathrm{~cm}$. de long; elles sont plus brusquement acuminécs et leur face inférieure est moins mate que celle des fenilles du 13. Lujae sur lesquelles les nervures secondaires sont particulièrement bien visibles formant un réseau ì mailles polygonales

Comme le montrent les proportions des feuilles $4-8 \times 2.4$ ct $6,5-13$ $\times 2,5.5$ chez les $B$. Gilletii et Lujae, les fenilles de eette dornière sont proportionnellement beaueoup plus allongćes.

Nous avons été heureux de pouvoir faire figurer un type de ce genre, peut-être rep̧résenté au Congo par d'autres espèces encore, car nous n'avions pu donner antéricurement des analyses du B. Gillelii. Cettc dernière espèce doit d'ailleur's être assez rare; car nous n'en avons plus reçu d'échantillons depuis 1900.

La comparaison des spécimens d'herbier fait supposer que les deux Bosqueiopsis doivent avoir un port assez différent. 



\section{INDEX}

DES

\section{ESPECES FIGUREES DANS LE TOME I \\ DISS}

Plantæ novæ vel minus cognitæ.

A. Espèces du Mozambique.

Angræcum filicornoides the Wild. nov. sper.

Angræcum tridactylites Rolfe

Asparagus Lujæ De Wild. nov. spec.

Cissampelos Wildemaniana $v . d$. Bossche nov. spec.

Dorstenia psilurus Welw. var. compacta De IVild. nov. spec.

Eulophia Kirkii Rolfe

Eulophia Lujæ De Wild. ncv. spec.

Gladiolus affinis De Wild. nov. spec.

Gladiolus morrumbalaensis De Wild. nov. spec.

Habenaria pedicellaris Reichb. fil.

Habenaria splendens Rendle.

Heinsenia Lujæ De Wild. nov. spec.

Kigelia Ikbaliæ De Wild. nov. spec. . . . .

Lissochilus morrumbalaensis De Wild. nov. spec.

Mystacidium appendiculatum De Wild. nov. spec.

Oncoba angustipetala De Wild. nov spec. .

Ottelia latifolia De Wild. nov. spec.

Oxalis morrumbalaensis $D e$ Wild. nov. spec.

Pavetta appendiculata De Wild. nov. spec.

Plectronia stipulata De Wild. nov. spec.

Polystachya Lujæ De Wild. nov. spec.

Polystachya uniflora De Wild.nov. spec. . .

Satyrium morrumbalaensis De Wild. nov. spee.

Triumfetta morrumbalana De IVild. nov. spec.

$\begin{array}{rr}\text { Pranchus } & \text { P..Ges } \\ \text { XXI } & 83 \\ \text { XX } & 79 \\ \text { XXXIV } & 157 \\ \text { II } & 5 \\ & \\ \text { LIV } & 233 \\ \text { XVI } & 65 \\ \text { XVII } & 69 \\ \text { XXXV } & 161 \\ \text { V } & 17 \\ \text { XII } & 49 \\ \text { XIII } & 57 \\ \text { III } & 9 \\ \text { I } & 1 \\ \text { XV } & 61 \\ \text { XXI } & 85 \\ \text { IV } & 13 \\ \text { XXXIII } & 153 \\ \text { XXXVI } & 163 \\ \text { XXXII } & 175 \\ \text { XXXVIII } & 171 \\ \text { XVIII } & 73 \\ \text { XIX } & 77 \\ \text { XIV } & 57 \\ \text { XXXVII } & 167\end{array}$


B. Espèces sud-africaines.

Aspalathus cliffortioides Bolus

$\begin{array}{rr}\text { PLACUE } & \text { PAgEs } \\ \text { XLII } & 193 \\ \text { XLIII } & 195 \\ \text { X } & 197 \\ \text { IX } & 39 \\ \text { VI } & 27 \\ \text { VII } & 30 \\ \text { VIII } & 33 \\ \text { VII } & 29 \\ \text { XLVI } & 205 \\ & 206 \\ \text { XLVII } & 209 \\ \text { XL } & 184 \\ & 183 \\ \text { XLI } & 187 \\ \text { XLV } & 203 \\ \text { XLVIII } & 213 \\ \text { XLIV } & 199 \\ \text { VI } & 23 \\ \text { XLIX } & 217 \\ \text { XI } & 43\end{array}$

Aspalathus leptoptera Bolus .

Aspalathus Schlechteri Bolus.

Carpha bracteosa $C$. B. Clarke

Carpha Scblechteri C. B. Clarke nov. spec.

Eleocharis Slechteri C. B. Clarlice

Ficinia distans C. B. Clarke nov spec

Ficinia minutiflora C. B. Clarke.

Ficinia mucronata C. B. Clarke nov. spec.

Gnidia pulvinata Bolus .

Gnidia transvaliensis Gilg. nov. spec. . . . .

Gymnostephium læve Bolus

Lotononis arenicola $R$. Sehleehter nov. spec.

Lotononis decipiens $R$. Scheehter nov. spec.

Lotononis delicatula Bolus nov. spec.

Lotononis Maximiliani R. Schlechter nov. spec. .

Muraltia spicata Bolus.

203

Phymaspermum appressum Bolus

Phyllosma capensis Bolus

Scirpus hemiuncialis C. B. Clarke

Senecio sociorum Bolus.

Tetraria ferruginea C. B. Clarke.

\section{Espèces du West Australia}

Adenanthos barbigera Lindl . . . . . . . /

Adenanthos Meissneri Lehm. . . . . . . .

XXYII

I 20

Conospermum Brownii Meissn.

XXXI I 40

Conospermum flexuosum $R$. $B r$.

XXXII 147

Conospermum floribundum Benth.

XXXI 142

Conospermum Huegelii $R$. $\mathrm{Br}$.

XXXII 147

Conospermum incurvum Lindl.

XXXI I 44

Isopogon asper $R . B r$.

XXIII 
Petrophila linearis $\mathrm{K}$. $\mathrm{Br}$.

IRTINCHES

I'AGES

Petrophila megaslostegia $F \cdot r$. Mucll.

XXV 109
110

Petrophila propinqua $R$. $B r$

Petrophila Serruriæ $R$. $B r$.

Petrophila Shuttelworthiana I/eissn.

Simsia abrotanoides $l \cdot v$. Muell.

Simsia latifolia $R$. Br . .

Simsia simplex $1 \cdot v$. Mucll

116

Synaphea favosa $R$. Br. .

XXV 112
114

XXVIII $1: 8$

XXIX 129

XXVIII 120

Synaphea pinnata $L$ indl.

$\mathrm{XXX}$

134

132

D. Espèces du Congo (District du Kasaij.

Bosqueiopsis Lujæ De Wild. nov. spec.

LII 239

Dorstenia Laurentii De Wild. var. brevipedicel-

lata De I'ild. nov. var.

LI $2: 25$

Dorste ia Lujæ De Wild. nov. spec.

L $2: 21$

Dorstenia psilurus Welw. .

LIII 229

Dorstenia scaphigera Bureau .

LII 227

Trymatococcus kamerunianus Engler.

$L Y \quad 235$ 



\title{
ENUUMERRATION
}

\author{
wes \\ PIANTES FIGURÉES \\ Classilis PAR
}

FAMILLES NATURELLES

Hydrocharitaceæ.

Ottelia latifolia De Wild. nov. spec.

PIANCHES P.AGES

XXXIII $15:$

\section{Cyperaceæ.}

Scirpus hemiuncialis C. B. Clarke nor. spec.

Elœocharis Schlechteri C. B. Clarke nov. spec.

Ficinia mucronata $C$. B. Clarke nov. spec.

Ficinia distans $C$. B. Clarke nov. spec.

Ficinia minutiflora $C$. B. Clarke.

Carpha Schlechteri $C$. B. Clarke nov. spec.

Carpha bracteosa $C$ B. Clarke

Tetraria ferruginea $C . B$. Clarke

$\begin{aligned} \text { VI } & 2: 3 \\ & 27 \\ \text { VII } & 29 \\ \text { VIII } & 30 \\ \text { IX } & 37 \\ \text { X } & 39 \\ \text { XI } & 43\end{aligned}$

\section{Liliaceæ.}

Asparagus Lujæ De Wild. nov. spec.

\section{Iridaceæ}

Gladiolus morrumbalaensis De Wild. nov. spec. Gladiolus affinis De Wild. nov. spec.

\section{Orchidaceæ.}

Habenaria pedicellaris Reichb. $f l$. . . . . . . . $\quad$ XII 49

Habenaria splendens Rendle . . . . . . . XII 53

Satyrium morrumbalaensis De Wild, nov. spec. XIV $5 \pi$

Lissochilus morrumbalıensis De Wild, nov. spec. $\mathrm{XV} \quad 61$ 
Eulophia Kirkii Rolfe

$\begin{array}{rr}\text { I'LINCHES } & \text { PIges } \\ \text { XVI } & 65 \\ \text { XVII } & 69 \\ \text { XVIII } & 7: 3 \\ \text { XIX } & 77 \\ \text { XX } & 79 \\ \text { XXI } & 83 \\ & 85\end{array}$

Eulophia Lujæ De Trild. nov. spec. .

Polystachya Lnjæ $D$.? W'ild, nov. spec. .

Polystachya uniflora De Wild nov. spec.

Allgræcum tridactylites Rolfe.

Angræcum filicornoides De Wild. nov. spee.

Mystacidinm appendiculatum I)e Wild. nov. spec.

Moraceæ

Dorstenia Lujæ De Wild nov, spec. . . . . . .

Dorstenia Laurentii the Wild. rar. brevipedicellata Isc Wild. nov. val.

L $\quad 221$

Dorstenia scaphigera bureau.

$\begin{aligned} \text { LI } & 225 \\ \text { LII } & 227 \\ \text { LIII } & 9 \cdot 20\end{aligned}$

Dorstenia psilurus Welv.

Id. id. il. var. compacta De Wild. nov. var.

LIV 233

Trymatococcus kamerunianus Engler.

LV 235

Bosqueiopsis Lujæ De Wild. nov. spec.

LVI 239

\section{Proteacere.}

Persoonia diadema $F v$. Mucll.

Persoonia longifolia $R$. $\mathrm{Br}$.

$\begin{array}{cr} & 92 \\ \text { XXII } & 94 \\ \text { XXIII } & 98 \\ & 100 \\ 102 \\ \text { XXIV } & 103 \\ & 105 \\ \text { XXV } & 109 \\ & 110 \\ & 112 \\ \text { XXVI } & 114 \\ & 116 \\ & 120 \\ \text { XXVII } & 122 \\ \text { XXVIII } & 126 \\ \text { XXVIII } & 128 \\ \text { XXIX } & 129 \\ \text { XXX } & 132 \\ & 134 \\ \text { XXXI } & 140 \\ & 142\end{array}$

Isopogon buxifolius $R$. $B r$.

Isopogon asper $R . B r$.

Isopogon tripartitus $R . B r$.

Isopogon teretifolius $R$. $B r$.

Isopogon Drummondii Benth.

Petrophila megalostegia $F, v$. Muell.

Petrophila linearis $R$. $B r$.

110

Petrophila Serruriæ $R$. $B r^{2}$.

Petrophila Shuttelworthiana Meissn.

Yetrophila propinqua $R$. $B r$. .

Adenanthos barbigera $\mathrm{Lindl}$.

Adenanthos Meissneri Lelim.

Simsia simplex $F$. v. Muell

Simsia abrotanoides $F$. v. Mucll.

Simsia latifolia $R$. Br. .

Synaphea pinnata $L$ indl.

Synaphea favosa $R$. Br.

Conospermum Brownei Meissn. .

Conospermum floribundum Benth.

\begin{tabular}{cc|} 
& 1 \\
& 1 \\
& 1 \\
$\cdot$ & 1 \\
$\cdot$ & $\cdot$ \\
$\cdot$ & $\cdot$ \\
$\cdot$ & $\cdot$ \\
$\cdot$ & $\cdot$ \\
$\cdot$ & \\
$\cdot$ & $\cdot$ \\
$\cdot$ & $\cdot$
\end{tabular}


Conospermum incurvum Lindl.

PI.ANrIL:S rimes

Conospermum Huegelii $R$. $B$ r .

XXX1 111

XXXil 1.15

Collospermum flexuosum li. lir.

X.I.11I 117

Menispermacere.

Cissampelos Wildemaniana v.d. Bossche nov. spec.

Leguminosæ

Lotononis decipiens $R$. Schlechler nov. spen:

Lotononis arenicola R. Schlechler nov. spres.

Lotononis delicatula Bolus nov. spees.

Lotononis Maximiliani $l$. schlechter

Aspalathus cliffortioides Bolus

Aspalathus leptoptera Bolus .

Aspalathus Schlecheteri Bolus

$\begin{array}{rr}\text { XL } & 183 \\ & 181 \\ \text { XLI } & 187 \\ 188 \\ \text { XLII } & 193 \\ \text { XLIII } & 195 \\ & 197\end{array}$

Oxalidaceæ.

Oxalis morrumbalaensis De IVild. nov. spee.

XXXVI 163

Rutaceæ.

Phyllosma capensis Bolus .

XLIV 199

Polygalaceæ

Muraltia spicata Bolus .

XLV 203

Tlliaceæ.

Triumfetta morrumbalana De IVild. nov. spec.

\section{Flacourtiaceæ}

Oncoba angustipetala the Wild. nov. spec.

II 13

Thymelæaceæ.

Gnidia pulvinata Bolus .

$\begin{array}{ll}\text { XII } & 205 \\ 206\end{array}$

Gnidia transvalensis Gilg nov. spec.

\section{Bignoniaceæ}

Kigelia Ikbaliæ De Wild. nov. spec. 
Rubiacæ.

Heinsenia Lujæ De Wild. nov. spec.

PLAXCIIES PAgES

Plectronia stipulata De Wild. nov. spec.

III

XXXVIII 171

Pavetta appendiculata De Wild. nov. spec.

XXXIX 175

\section{Compositæ.}

Gymnostephium læve Bolus .

XLVII 209

Phymaspermum appressum Bolus

XLVIII 213

Senecio sociorum Bolus

XLIX 217 
. 


$$
\left(y^{3}\right)^{3}
$$






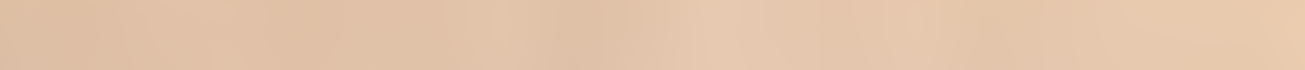




\section{Date Due}

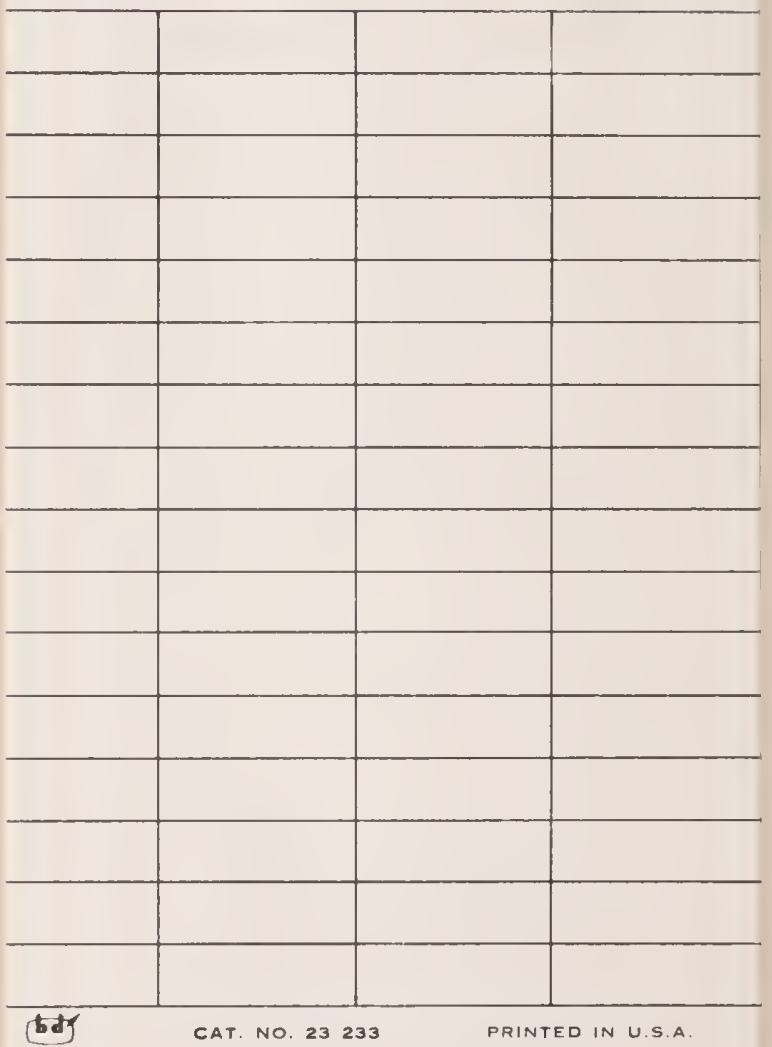




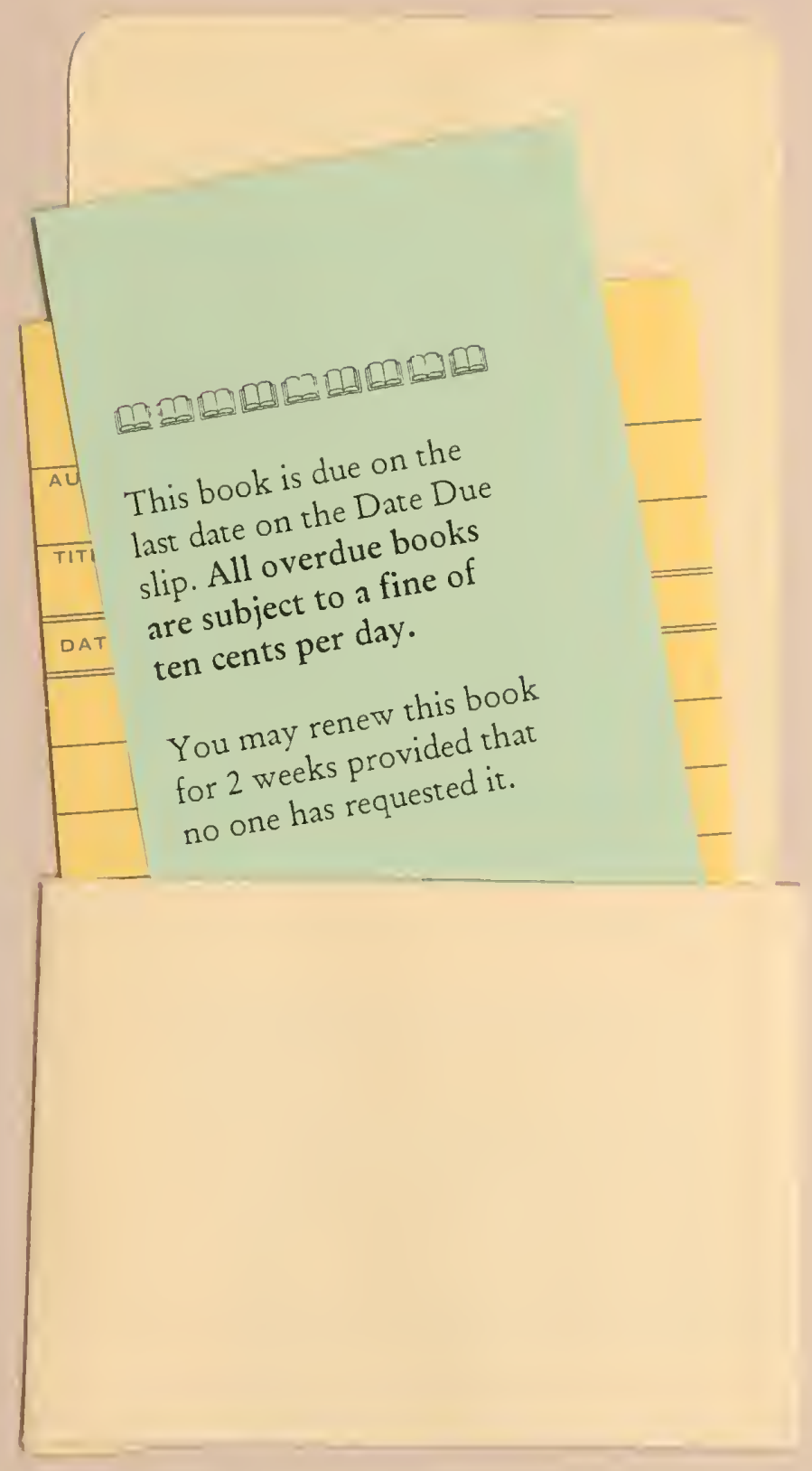




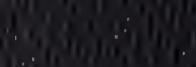

ing

$10\left(\begin{array}{l}1 \\ 0\end{array}\right.$

of

Q

告,

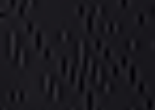

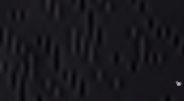

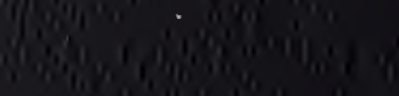

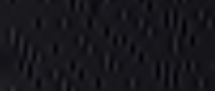

M.

(1)

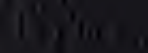

6.,

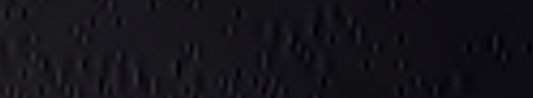

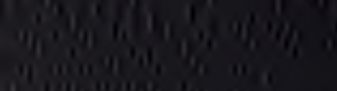

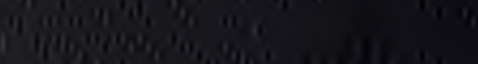

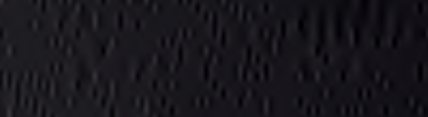

(III)

Q

(1,

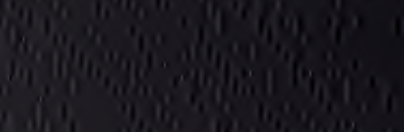

